\title{
Efficient Strategy for the Construction of Both Enantiomers of the Octahydropyrroloquinolinone Ring System: Total Synthesis of (+)- Aspidospermidine
}

Ganesh Pandey,* Shiva Kumar Burugu and Pushpendra Singh

\author{
Molecular synthesis and Drug Discovery Laboratory \\ Centre of Biomedical Research, SGPGIMS Campus \\ Lucknow- 226014, India.
}

Email: gp.pandey@cbmr.res.in

\section{Supporting Information}

I. General Information

II. Experimental Procedures and Characterization data.

III. ${ }^{1} \mathrm{H}$ and ${ }^{13} \mathrm{C}$ NMR spectra of compounds and other analytical data. 


\section{General Information.}

All anhydrous reactions were performed under argon atmosphere and hot air oven dried glassware $\left(110^{\circ} \mathrm{C}\right)$ were used. Dry tetrahydrofuran $(\mathrm{THF})$ and diethyl ether $\left(\mathrm{Et}_{2} \mathrm{O}\right)$ were obtained by passing commercially available pre-dried, oxygen-free formulations through activated alumina columns and dried by distillation over sodium / benzophenone. Benzene, toluene, acetonitrile and dichloromethane (DCM) were distilled over calcium hydride and stored over $4 \AA$ molecular sieves. Triethylamine (TEA) was distilled over potassium hydroxide. All the solvents used for chromatography were distilled at respective boiling points using known procedures.

All commercial reagents were obtained from Sigma-Aldrich and Spectrochem, India. Progress of the reactions was monitored by thin layer chromatography $(0.25 \mathrm{~mm}$ Merck 60F254 silica gel plates) and visualized by using UV light, ethanolic solution of phosphomolybdic acid, iodine and $\mathrm{KMnO}_{4}$ solution. Column chromatography was performed on silica gel 60-120/100-200/230-400 mesh obtained from Spectrochem Fine Chemical Co. India or LOBA India. Typical syringe and cannula techniques were used to transfer air and moisture sensitive reagents.

All melting points were recorded on a BUCHI Melting point M-560 apparatus and were uncorrected in degree Celsius. IR spectra were recorded on a Perkin-Elmer FT-Infrared Spectrometer. ${ }^{1} \mathrm{H}$ and ${ }^{13} \mathrm{C}$ NMR spectra were recorded on BRUKER 800 UltraShield PLUS and BRUKER 400 ULTRA SHIELD instruments operating at $800 \mathrm{MHz}, 400 \mathrm{MHz}$ and $201 \mathrm{MHz}, 101$ $\mathrm{MHz}$ respectively using deuteriated solvent. Chemical shifts are reported in $\delta \mathrm{ppm}$. Proton coupling constants $(J)$ are reported as absolute values in $\mathrm{Hz}$ and multiplicity (br, broadened; s, singlet; d, doublet; t, triplet; dd, doublet of doublet; dt, doublet of triplet; td, triplet of doublet; m, multiplet). ${ }^{13} \mathrm{C}$ NMR chemical shifts are reported in ppm relative to the central line of $\mathrm{CDCl}_{3}(\delta$ 77.00). Electro spray ionization (ESI) mass spectrometry (MS) experiments were performed on Agilent Technologies 6530 Accurate-Mass Q-TOF LC/MS. Optical rotations were measured on a Digipol 781 M6U Automatic Polarimeter. HPLC were performed on Agilent Technologies 1260 Infinity. 


\section{Experimental Procedures and Characterization data.}

\section{Diethyl (1-(4-methoxybenzyl)-2-oxopiperidin-3-yl)phosphonate (11) :}

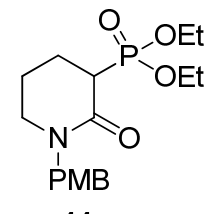

11

To a cooled $\left(-78^{\circ} \mathrm{C}\right)$ stirring solution of 1-(4-methoxybenzyl) piperidin-2-one (10) (15.0 g, 0.068 mol) in THF (200 mL), LiHMDS (75 mL, 1M) solution was added. After $1 \mathrm{~h}$ of stirring, diethyl chlorophosphate $(10.9 \mathrm{~mL}, 0.075 \mathrm{~mol})$ was added drop wise to the reaction mixture. The reaction mixture was slowly allowed to warm to room temperature and was continued for overnight. The reaction mixture was quenched with saturated aqueous solution of ammonium Chloride, extracted with EtOAc $(3 \times 200 \mathrm{~mL})$. The combined organic layer was washed with brine and dried over $\mathrm{Na}_{2} \mathrm{SO}_{4}$, concentrated. The crude compound was purified by column chromatography $\left(\mathrm{SiO}_{2}\right.$, ethylacetate - hexane, $3: 7 \rightarrow 10: 0)$ to obtain $11(16.6 \mathrm{~g}, 68 \%)$ as a light yellow oil.

TLC: $\mathrm{R}_{f}=0.45$ (ethylacetate $:$ hexane $=8: 2$ ).

IR (neat): 2981, 2935, 2910, 1636, 1512, 1491, 1443, 1296, 1246, 1175, 1028, $967 \mathrm{~cm}^{-1}$.

${ }^{1} \mathbf{H}$ NMR (800 MHz, CDCl 3$): \delta 7.21(\mathrm{~d}, J=8.6 \mathrm{~Hz}, 2 \mathrm{H}), 6.85(\mathrm{~d}, J=8.6 \mathrm{~Hz}, 2 \mathrm{H}), 4.64(\mathrm{~d}, J=$ $14.9 \mathrm{~Hz}, 1 \mathrm{H}), 4.49$ (d, J=14.5 Hz, 1H), 4.29 - 4.09 (m, 4H), 3.79 (s, 3H), 3.28-3.24 (m, 1H), $3.21-3.15(\mathrm{~m}, 1 \mathrm{H}), 3.10-3.02(\mathrm{~m}, 1 \mathrm{H}), 2.24-2.16(\mathrm{~m}, 1 \mathrm{H}), 2.13-2.01(\mathrm{~m}, 2 \mathrm{H}), 1.72$ - $1.66(\mathrm{~m}$, 1H), $1.36(\mathrm{t}, J=7.1 \mathrm{~Hz}, 3 \mathrm{H}), 1.32(\mathrm{t}, J=7.1 \mathrm{~Hz}, 3 \mathrm{H})$.

${ }^{13}$ C NMR (101 MHz, CDCl $): \delta 164.9,158.9,129.3,129.0,114.0,63.0,62.0,55.2,49.8,46.9$, $42.5,41.1,23.2,21.4,16.4,16.3$.

HRMS (ESI): Calculated for $[\mathrm{M}+\mathrm{Na}]^{+} \mathrm{C}_{17} \mathrm{H}_{26} \mathrm{NO}_{5} \mathrm{PNa}: 378.1446$, found: 378.1457. 


\section{Wittig- Horner Olefination Reaction:}

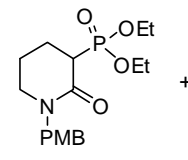

11

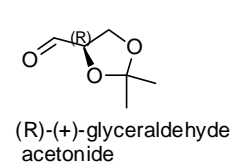

acetonide

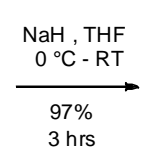

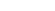

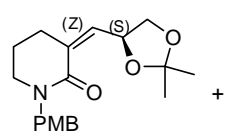

12

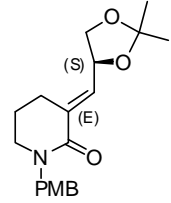

13

To a stirred solution of $\mathrm{NaH}(2.48 \mathrm{~g}, 61.91 \mathrm{mmol}, 60 \%$ dispersion in oil $)$ in $\mathrm{THF}(75 \mathrm{~mL})$ at $0{ }^{\circ} \mathrm{C}$, was added diethyl (1-(4-methoxybenzyl)-2-oxopiperidin-3-yl)phosphonate (11) (20.0 g, 56.28 mmol) in THF $(75 \mathrm{~mL})$. After $15 \mathrm{~min}$, a solution of $(R)-(+)$-glyceraldehyde acetonide $(7.69 \mathrm{~g}$, $59.09 \mathrm{mmol})$ in THF $(50 \mathrm{~mL})$ was added drop wise into the flask. The reaction mixture was allowed to warm to room temperature and further stirred for 3 hours, and quenched with saturated $\mathrm{NH}_{4} \mathrm{Cl}$ solution. The mixture was extracted with ethylacetate $(150 \mathrm{~mL} \times 3)$, dried over $\mathrm{Na}_{2} \mathrm{SO}_{4}$ and concentrated. The crude was purification by column chromatography (using ethylacetate hexane 1:9 $\rightarrow 3: 7$ gradient) afforded pure 12 and $\mathbf{1 3}$ in 4:6 ratio with combined yield of $97 \%$ (18.1 g).

(S,Z)-3-((2,2-dimethyl-1,3-dioxolan-4-yl)methylene)-1-(4-methoxybenzyl) piperidin-2-one (12):

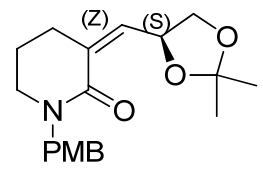

12

Yield: (7.23 g, 39\%); light yellow color oil.

TLC: $\mathrm{R}_{f}=0.6$ (ethylacetate $:$ hexane $=3: 7$ ).

$[\boldsymbol{\alpha}]_{\mathbf{D}^{\mathbf{2 6}}}=+104.94\left(\mathrm{CHCl}_{3}, c=0.79\right)$.

IR (neat): 3439, 3008, 2936, 1733, 1662, 1613, 1512, $1247 \mathrm{~cm}^{-1}$. 
${ }^{1}$ H NMR (800 MHz, $\left.\mathbf{C D C l}_{3}\right): \delta 7.17(\mathrm{~d}, J=7.4 \mathrm{~Hz}, 2 \mathrm{H}), 6.86(\mathrm{~d}, J=7.4 \mathrm{~Hz}, 2 \mathrm{H}), 5.93(\mathrm{~d}, J=$ $6.3 \mathrm{~Hz}, 1 \mathrm{H}), 5.59-5.56(\mathrm{~m}, 1 \mathrm{H}), 4.67(\mathrm{~d}, J=14.5 \mathrm{~Hz}, 1 \mathrm{H}), 4.64(\mathrm{t}, J=7.7 \mathrm{~Hz}, 1 \mathrm{H}), 4.42(\mathrm{~d}, J=$ $14.5 \mathrm{~Hz}, 1 \mathrm{H}), 3.79(\mathrm{~s}, 3 \mathrm{H}), 3.68-3.63(\mathrm{~m}, 1 \mathrm{H}), 3.23(\mathrm{t}, J=5.9 \mathrm{~Hz}, 2 \mathrm{H}), 2.54-2.43(\mathrm{~m}, 2 \mathrm{H})$, $1.90-1.84(\mathrm{~m}, 1 \mathrm{H}), 1.83-1.75(\mathrm{~m}, 1 \mathrm{H}), 1.47$ (s, 3H), 1.39 (s, 3H).

${ }^{13}$ C NMR (101 MHz, $\left.\mathbf{C D C l}_{3}\right): \delta 164.0,158.9,140.4,130.3,129.2,129.1,113.9,109.0,74.4$, $70.2,55.2,49.4,47.1,31.6,26.7,25.3,23.2$.

HRMS (ESI): Calculated for [M + Na ${ }^{+} \mathrm{C}_{19} \mathrm{H}_{25} \mathrm{NO}_{4} \mathrm{Na}$ : 354.1681 , found: 354.1675 .

(S,E)-3-((2,2-dimethyl-1,3-dioxolan-4-yl)methylene)-1-(4-methoxybenzyl) piperidin-2-one (13):

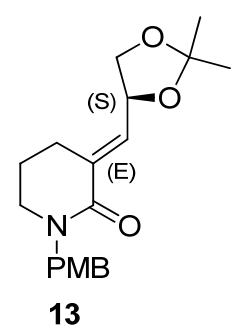

Yield: (10.87 g, 58\%); light yellow color oil.

TLC: $\mathrm{R}_{f}=0.4$ (ethylacetate $:$ hexane $=3: 7$ ).

$[\boldsymbol{\alpha}] \mathbf{D}^{\mathbf{2 6}}=-9.58\left(\mathrm{CHCl}_{3}, \mathrm{c}=1\right)$.

IR (film): 3440, 2986, 2935, 1665, 1613, 1512, $1246 \mathrm{~cm}^{-1}$.

${ }^{1} \mathbf{H}$ NMR (800 MHz ,CDCl $\left.\mathbf{~}_{3}\right): \delta 7.20(\mathrm{~d}, J=7.4 \mathrm{~Hz}, 2 \mathrm{H}), 6.88-6.82(\mathrm{~m}, 3 \mathrm{H}), 4.85$ - $4.78(\mathrm{~m}$, $1 \mathrm{H}), 4.63(\mathrm{~d}, J=14.1 \mathrm{~Hz}, 1 \mathrm{H}), 4.56(\mathrm{~d}, J=14.5 \mathrm{~Hz}, 1 \mathrm{H}), 4.12(\mathrm{t}, J=7.5 \mathrm{~Hz}, 1 \mathrm{H}), 3.79(\mathrm{~s}, 3 \mathrm{H})$, $3.69(\mathrm{t}, J=7.9 \mathrm{~Hz}, 1 \mathrm{H}), 3.29-3.23(\mathrm{~m}, 2 \mathrm{H}), 2.73-2.65(\mathrm{~m}, 1 \mathrm{H}), 2.46-2.37(\mathrm{~m}, 1 \mathrm{H}), 1.81-1.78$ $(\mathrm{m}, 2 \mathrm{H}), 1.44(\mathrm{~s}, 3 \mathrm{H}), 1.41(\mathrm{~s}, 3 \mathrm{H})$.

${ }^{13}$ C NMR (101 MHz, $\left.\mathbf{C D C l}_{3}\right): \delta 163.8,158.9,134.3,132.6,129.4,129.2,113.9,109.6,72.2$, $68.7,55.2,50.4,46.7,26.5,25.9,25.0,22.5$.

HRMS (ESI): Calculated for [M + Na ${ }^{+} \mathrm{C}_{19} \mathrm{H}_{25} \mathrm{NO} 4 \mathrm{Na}$ : 354.1681, found: 354.1676. 


\section{General procedure for acetonide deprotection:}

To a stirred solution of acetonide compound (15 g, $45.26 \mathrm{mmol})$ in THF (75 mL) was added glacial acetic acid $(75 \mathrm{~mL})$ followed by water $(75 \mathrm{~mL})$ at room temperature. The resulting mixture was heated at $60{ }^{\circ} \mathrm{C}$ for overnight. The reaction mixture allowed to cool to room temperature, concentrated to dryness and the crude was neutralized with minimum amount of saturated aqueous $\mathrm{NaHCO}_{3}$ solution. The reaction mixture was extracted with ethyl acetate $(3 \times 200 \mathrm{~mL})$, dried over $\mathrm{Na}_{2} \mathrm{SO}_{4}$ and concentrated. Purification by column chromatography (using ethyl acetate - hexane $2: 8 \rightarrow 10: 0$ gradient) resulted Diol in excellent Yield.

\section{(S, Z)-3-(2,3-dihydroxypropylidene)-1-(4-methoxybenzyl)piperidin-2-one :}

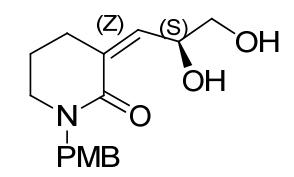

Yield: $12.5 \mathrm{~g}, 95 \%$.

TLC: $R_{f}=0.4$ (ethyl acetate $:$ hexane $=8: 2$ ).

white solid, $\mathrm{mp}=95-96^{\circ} \mathrm{C}$.

$[\boldsymbol{\alpha}] \mathbf{D}^{\mathbf{2 3}}=-61.38\left(\mathrm{CHCl}_{3}, c=1.03\right)$.

IR (film): 3380, 2932, 1610, 1511, 1490, $1246 \mathrm{~cm}^{-1}$.

${ }^{1} \mathbf{H}$ NMR (400 MHz, CDCl $): \delta 7.18(\mathrm{~d}, J=8.6 \mathrm{~Hz}, 2 \mathrm{H}), 6.85(\mathrm{~d}, J=8.6 \mathrm{~Hz}, 2 \mathrm{H}), 5.96(\mathrm{~d}, J=$ $6.0 \mathrm{~Hz}, 1 \mathrm{H}), 5.60$ (br s, 1H), $4.78-4.70(\mathrm{~m}, 1 \mathrm{H}), 4.57(\mathrm{~s}, 2 \mathrm{H}), 3.79(\mathrm{~s}, 3 \mathrm{H}), 3.76-3.61(\mathrm{~m}, 2 \mathrm{H})$, $3.30-3.22$ (t, $J=6.2 \mathrm{~Hz}, 2 \mathrm{H}), 3.25$ (br s, $1 \mathrm{H}), 2.52-2.48$ (t, $J=6.0 \mathrm{~Hz}, 2 \mathrm{H}), 1.89-1.77$ (m, $2 \mathrm{H})$.

${ }^{13}$ C NMR (101 MHz, CDCl 3 ): $\delta$ 165.3, 159.0, 139.8, 132.3, 129.4, 128.7, 114.0, 68.8, 66.0, $55.2,49.9,47.3,32.2,23.0$.

HRMS (ESI): Calculated for $[\mathrm{M}+\mathrm{Na}]^{+} \mathrm{C}_{16} \mathrm{H}_{21} \mathrm{NNaO}_{4}: 314.1368$, found: 314.1361 . 
(S, E)-3-(2,3-dihydroxypropylidene)-1-(4-methoxybenzyl)piperidin-2-one :

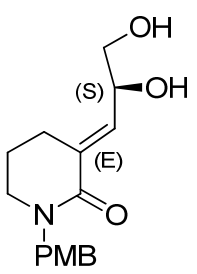

TLC: $\mathrm{R}_{f}=0.25$ (ethyl acetate).

Yield: $12.1 \mathrm{~g}, 92 \%$, as white solid $\left(\mathrm{mp}=85-87^{\circ} \mathrm{C}\right)$.

$[\boldsymbol{\alpha}] \mathbf{D}^{24}=+11.31\left(\mathrm{CHCl}_{3}, c=0.7\right)$.

IR (film): 3374, 2930, 1659, 1600, 1511, 1488, $1246 \mathrm{~cm}^{-1}$.

${ }^{1}$ H NMR (400 MHz, CDCl $): \delta 7.19(\mathrm{~d}, J=7.6 \mathrm{~Hz}, 2 \mathrm{H}), 6.89-6.81(\mathrm{~m}, 3 \mathrm{H}), 4.64-4.47(\mathrm{~m}, 3 \mathrm{H})$, 3.79 (s, 3H), 3.65 - 3.58 (m, 2H), 3.39 (br s, 1H), 3.29-3.23 (m, 2H), 2.99 (br s, 1H), 2.72-2.58 (m, 1H), $2.54-2.39(\mathrm{~m}, 1 \mathrm{H}), 1.82-1.78(\mathrm{~m}, 2 \mathrm{H})$.

${ }^{13}$ C NMR (101 MHz, CDCl 3$): \delta 164.5,159.0,136.4,131.5,129.4,129.0,114.0,69.2,65.7$, $55.2,50.5,46.9,25.0,22.4$.

HRMS (ESI): Calculated for $[\mathrm{M}+\mathrm{Na}]^{+} \mathrm{C}_{16} \mathrm{H}_{21} \mathrm{NO}_{4} \mathrm{Na}: 314.1368$, found: 314.1358 .

\section{General procedure for selective -OTBS protection:}

To a stirred solution of diol $(10 \mathrm{~g}, 34.32 \mathrm{mmol})$ and imidazole $(2.80 \mathrm{~g}, 41.19 \mathrm{mmol})$ in dichloromethane $(200 \mathrm{~mL})$, a solution of TBSCl $(5.43 \mathrm{~g}, 36.04 \mathrm{mmol})$ in dichloromethane was added over $15 \mathrm{~min}$ at $-15^{\circ} \mathrm{C}$ and stirring was continued for $2 \mathrm{~h}$. Progress of the reaction was monitored by TLC. On consumption of starting material, the reaction mixture was quenched with water, extracted with dichloromethane. The combined organic layer was dried over $\mathrm{Na}_{2} \mathrm{SO}_{4}$, concentrated under reduced pressure. The resultant residue was purified by column chromatography using ethylacetate in hexane which resulted respective silylether in excellent yield. 
(S,Z)-3-(3-((tert-butyldimethylsilyl)oxy)-2-hydroxypropylidene)-1-(4-

methoxybenzyl)piperidin-2-one (14):

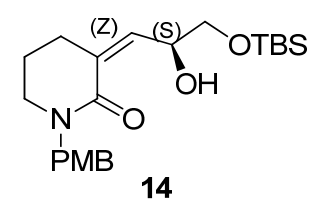

TLC: $\mathrm{R}_{f}=0.4$ (ethyl acetate: hexane $=3: 7$ ).

Yield: $13.7 \mathrm{~g}, 98 \%$.

white solid, $\mathrm{mp}=102-104{ }^{\circ} \mathrm{C}$.

$[\boldsymbol{\alpha}]_{\mathbf{D}} \mathbf{D}^{25}=-10.59\left(\mathrm{CHCl}_{3}, \mathrm{c}=1.02\right)$.

IR (film): 3369, 3016, 2954, 1610, 1512, 1459, $1246 \mathrm{~cm}^{-1}$.

${ }^{1} \mathbf{H}$ NMR (400MHz, $\left.\mathbf{C D C l}_{3}\right): \delta 7.19(\mathrm{~d}, J=7.8 \mathrm{~Hz}, 2 \mathrm{H}), 6.85(\mathrm{~d}, J=7.8 \mathrm{~Hz}, 2 \mathrm{H}), 6.00(\mathrm{~d}, J=5.3$ Hz, 1H), 5.05 (br s, 1H), 4.84-4.75 (m, 1H), 4.62 (d, $J=14.4 \mathrm{~Hz}, 1 \mathrm{H}), 4.52$ (d, $J=14.4 \mathrm{~Hz}, 1 \mathrm{H})$, $3.79(\mathrm{~s}, 3 \mathrm{H}), 3.77-3.67(\mathrm{~m}, 2 \mathrm{H}), 3.25(\mathrm{t}, J=5.5 \mathrm{~Hz}, 2 \mathrm{H}), 2.52-2.45(\mathrm{~m}, 2 \mathrm{H}), 1.92-1.76(\mathrm{~m}, 2 \mathrm{H})$, $0.91(\mathrm{~s}, 9 \mathrm{H}), 0.09(\mathrm{~s}, 6 \mathrm{H})$.

${ }^{13}$ C NMR (101MHz, $\left.\mathbf{C D C l}_{3}\right): \delta$ 165.0, 159.0, 141.2, 131.2, 129.4, 129.0, 114.0, 68.8, 66.3, 55.2, $49.7,47.3,32.3,25.9,23.1,18.3,-5.3,-5.2$.

HRMS (ESI): Calculated for [M + Na] ${ }^{+} \mathrm{C}_{22} \mathrm{H}_{35} \mathrm{NO}_{4} \mathrm{SiNa}: 428.2233$, found : 428.2236 .

(S,E)-3-(3-((tert-butyldimethylsilyl)oxy)-2-hydroxypropylidene)-1-(4methoxybenzyl)piperidin-2-one (9):

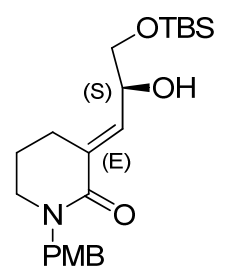

9 
Yield: $13.5 \mathrm{~g}, 97 \%$.

White solid, $\mathrm{mp}=105-106^{\circ} \mathrm{C}$.

TLC: $\mathrm{R}_{f}=0.4\left(\mathrm{SiO}_{2}\right.$, ethylacetate $:$ hexane $\left.=1: 1\right)$.

$[\boldsymbol{\alpha}]_{\mathbf{D}} \mathbf{2 5}=-4.97\left(\mathrm{CHCl}_{3}, c=1.17\right)$.

IR (film): 3416, 2928, 2856, 1601, 1494, $1253 \mathrm{~cm}^{-1}$.

${ }^{1} \mathbf{H}$ NMR (400MHz, $\left.\mathbf{C D C l}_{3}\right): \delta 7.20(\mathrm{~d}, J=8.1 \mathrm{~Hz}, 2 \mathrm{H}), 6.84(\mathrm{~d}, J=8.1 \mathrm{~Hz}, 2 \mathrm{H}), 6.79$ (d, $J=7.8$ $\mathrm{Hz}, 1 \mathrm{H}), 4.63$ (d, $J=14.5 \mathrm{~Hz} 1 \mathrm{H}), 4.56$ (d, $J=14.5 \mathrm{~Hz}, 1 \mathrm{H}), 4.43$ - 4.52 (m, 1H), 3.79 (s, 3H), 3.65 - $3.52(\mathrm{~m}, 2 \mathrm{H}), 3.26(\mathrm{t}, J=5.4 \mathrm{~Hz}, 2 \mathrm{H}), 2.76-2.65(\mathrm{~m}, 1 \mathrm{H}), 2.62$ (br s, 1H), 2.52 - 2.38 (m, 1H), $1.76-1.87(\mathrm{~m}, 2 \mathrm{H}), 1.68(\mathrm{~m}, 1 \mathrm{H}), 0.91(\mathrm{~s}, 9 \mathrm{H}), 0.09$ (s, 6H).

${ }^{13}$ C NMR (101MHz, $\left.\mathbf{C D C l}_{3}\right): \delta$ 164.1, 158.9, 134.9, 132.4, 129.4, 129.3, 113.9, 69.0, 66.0, 55.2, $50.4,46.8,25.9,25.3,22.6,18.3,-5.4$.

HRMS (ESI): Calculated for $[\mathrm{M}+\mathrm{Na}]^{+} \mathrm{C}_{22} \mathrm{H}_{35} \mathrm{NO}_{4} \mathrm{SiNa}: 428.2233$, found : 428.2233 .

\section{Johnson-Claisen rearrangement:}

To a solution of $9(10 \mathrm{~g}, 24.65 \mathrm{mmol})$ in triethyl orthoacetate $(150 \mathrm{~mL})$, was added catalytic amount of propionic acid $(0.2 \mathrm{~mL}, 2.71 \mathrm{mmol})$ under argon atmosphere. The resulting mixture was heated at $130{ }^{\circ} \mathrm{C}$ for $8 \mathrm{~h}$. The reaction was monitored by TLC. After complete consumption of starting material, the reaction mixture was concentrated under reduced pressure. The resulting residue was purified by column chromatography using ethyl acetate and hexane $(1: 9 \rightarrow 2: 8$ gradient $)$ to obtain $\mathbf{8 b}$ as a colorless liquid.

(S, E)-ethyl 2-(3-(3-((tert-butyldimethylsilyl)oxy)prop-1-en-1-yl)-1-(4-methoxybenzyl)-2oxopiperidin-3-yl)acetate (8b):

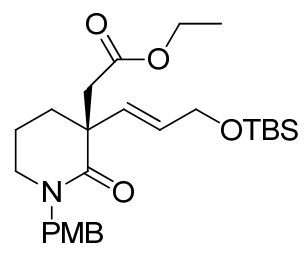

$8 b$ 
Colorless liquid; Yield: (11.4 g, 97\%).

TLC: $\mathrm{R}_{f}=\mathbf{0 . 5 5}\left(\mathrm{SiO}_{2}\right.$, ethyl acetate: hexane $\left.=3: 7\right)$.

$[\boldsymbol{\alpha}]_{\mathbf{D}}{ }^{24}=-15.38\left(c=0.84, \mathrm{CHCl}_{3}\right)$.

HPLC : Chiralcel OD-H Column; $1 \mathrm{~mL} / \mathrm{min}$ flow rate; IPA: $\mathrm{n}-H e x a n e 10: 90 ; \lambda=210 \mathrm{~nm}$, $>99 \%$ ee; retention time $7.2 \mathrm{~min}$.

IR (film): 2953, 2931, 2856, 1734, 1640, 1512, 1462, $1249 \mathrm{~cm}^{-1}$.

${ }^{1}$ H NMR (400MHz, $\left.\mathbf{C D C l}_{3}\right): \delta 7.22(\mathrm{~m}, J=7.8 \mathrm{~Hz}, 2 \mathrm{H}), 6.85(\mathrm{~m}, J=7.8 \mathrm{~Hz}, 2 \mathrm{H}), 5.66$ (br s, 2H), $4.72(\mathrm{~d}, J=14.4 \mathrm{~Hz}, 1 \mathrm{H}), 4.39$ (d, $J=14.4 \mathrm{~Hz}, 1 \mathrm{H}), 4.21-4.15$ (m, 2H), 4.15 - 4.06 (m, 2H), 3.80 (s, 3H), $3.29(\mathrm{td}, J=11.2,3.9 \mathrm{~Hz}, 1 \mathrm{H}), 3.20(\mathrm{~d}, J=16.5 \mathrm{~Hz}, 1 \mathrm{H}), 3.18-3.09$ (m, 1H), $2.38(\mathrm{~d}, J=$ $16.4 \mathrm{~Hz}, 1 \mathrm{H}), 2.19(\mathrm{t}, J=13.2 \mathrm{~Hz}, 1 \mathrm{H}), 1.97-1.75(\mathrm{~m}, 2 \mathrm{H}), 1.68(\mathrm{~m}, 1 \mathrm{H}), 1.24(\mathrm{t}, J=7.3 \mathrm{~Hz}, 3 \mathrm{H})$, $0.91(\mathrm{~s}, 9 \mathrm{H}), 0.06(\mathrm{~s}, 6 \mathrm{H})$.

${ }^{13}$ C NMR (101MHz, $\left.\mathbf{C D C l} 3\right): \delta 171.7,171.4,158.8,133.2,130.0,129.6,129.4,113.8,63.5,60.2$, $55.2,49.9,47.3,46.2,43.2,31.1,25.9,19.4,18.3,14.2,-5.2$.

HRMS (ESI): Calculated for $[\mathrm{M}+\mathrm{Na}]^{+} \mathrm{C}_{26} \mathrm{H}_{41} \mathrm{NNaO}_{5} \mathrm{Si}: 498.2652$, found : 498.2656 .

(R,E)-ethyl 2-(3-(3-((tert-butyldimethylsilyl)oxy)prop-1-en-1-yl)-1-(4-methoxybenzyl)-2oxopiperidin-3-yl)acetate (8a):

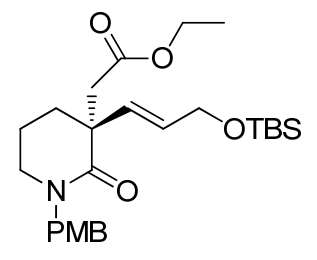

$8 \mathbf{a}$

Yield: (11.2 g, 95\%); colorless liquid.

TLC: $\mathrm{R}_{f}=\mathbf{0 . 5 5}\left(\mathrm{SiO}_{2}\right.$, ethyl acetate: hexane $\left.=3: 7\right)$.

$[\boldsymbol{\alpha}]_{\mathbf{D}} \mathbf{2 4}^{4}=+12.19\left(c=0.31, \mathrm{CHCl}_{3}\right)$.

HPLC : Chiralcel OD-H Column; $1 \mathrm{~mL} / \mathrm{min}$ flow rate; IPA: $\mathrm{n}-H e x a n e ~ 10: 90 ; \lambda=210 \mathrm{~nm},>\mathbf{9 9 \%}$ ee; retention time $6.0 \mathrm{~min}$. 
${ }^{1}$ H NMR (400MHz, $\left.\mathbf{C D C l}_{3}\right): \delta 7.22(\mathrm{~d}, J=8.3 \mathrm{~Hz}, 2 \mathrm{H}), 6.84$ (d , $\left.J=8.3 \mathrm{~Hz}, 2 \mathrm{H}\right), 5.66$ (br s, 2H), $4.71(\mathrm{~d}, J=14.4 \mathrm{~Hz}, 1 \mathrm{H}), 4.38(\mathrm{~d}, J=14.6 \mathrm{~Hz}, 1 \mathrm{H}), 4.24$ - $4.16(\mathrm{~m}, 2 \mathrm{H}), 4.16$ - 4.05 (m, 2H), 3.79 (s, 3H), 3.29 (td, $J=11.6 \mathrm{~Hz}, 3.8 \mathrm{~Hz}, 1 \mathrm{H}), 3.2(\mathrm{~d}, J=16.6 \mathrm{~Hz}, 1 \mathrm{H}), 3.18-3.08$ (m, 1H), 2.38 (d, $J$ $=16.6 \mathrm{~Hz}, 1 \mathrm{H}), 2.19(\mathrm{td}, J=12.7 \mathrm{~Hz}, 3.1 \mathrm{~Hz} 1 \mathrm{H}), 1.94-1.77(\mathrm{~m}, 2 \mathrm{H}), 1.68(\mathrm{~d}, J=12.6 \mathrm{~Hz}, 1 \mathrm{H})$, $1.23(\mathrm{t}, J=7.1 \mathrm{~Hz}, 3 \mathrm{H}), 0.90(\mathrm{~s}, 9 \mathrm{H}), 0.06(\mathrm{~s}, 6 \mathrm{H})$.

${ }^{13}$ C NMR (101MHz, $\left.\mathbf{C D C l}_{3}\right): \delta 171.6,171.4,158.8,133.1,130.0,129.6,129.4,113.8,63.5,60.2$, $55.2,49.9,47.3,46.1,43.2,31.0,25.9,19.4,18.3,14.2,-5.2$.

HRMS (ESI): Calculated for $[\mathrm{M}+\mathrm{Na}]^{+} \mathrm{C}_{26} \mathrm{H}_{41} \mathrm{NO}{ }_{5} \mathrm{SiNa}: 498.2652$, found : 498.2655 .

\section{Reduction of Ester moiety to Aldehyde:}

To a solution of $8(1.0 \mathrm{~g}, 2.1 \mathrm{mmol})$ in anhydrous dichloromethane $(15 \mathrm{~mL})$, DIBAL-H $(3.1 \mathrm{~mL}$, $3.1 \mathrm{mmol}, 1 \mathrm{M}$ solution in hexane) was added at $-78^{\circ} \mathrm{C}$. After $2 \mathrm{~h}$, the reaction was quenched by the addition of a saturated aqueous solution of potassium sodium tartrate $(15 \mathrm{~mL})$. The reaction mixture was stirred at room temperature for $1 \mathrm{~h}$. The aqueous layer was extracted with dichloromethane $(3 \times 15 \mathrm{~mL})$. The combined organic layer was dried over $\mathrm{Na}_{2} \mathrm{SO}_{4}$, concentrated and purified by column chromatography which afforded corresponding aldehyde.

(S,E)-2-(3-(3-((tert-butyldimethylsilyl)oxy)prop-1-en-1-yl)-1-(4-methoxybenzyl)-2oxopiperidin-3-yl)acetaldehyde:

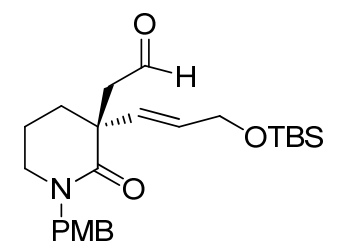

TLC: $\mathrm{R}_{f}=\mathbf{0 . 5}\left(\mathrm{SiO}_{2}\right.$, ethylacetate $:$ hexane $\left.=3: 7\right)$, as colorless liquid.

Yield: $(0.72 \mathrm{~g}, 80 \%)$, colorless liquid.

$[\boldsymbol{\alpha}] \mathbf{D}^{\mathbf{2 4}}=+3.78\left(c=1, \mathrm{CHCl}_{3}\right)$.

IR (film): 2952, 2931, 2856, 2897, 1718, 1633, 1512, 1462, 1442, 1357, $1249 \mathrm{~cm}^{-1}$.

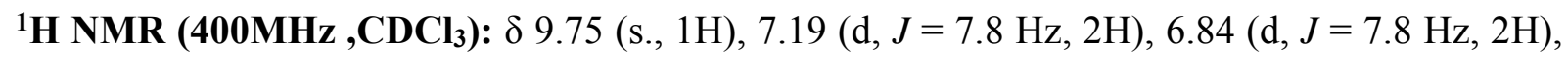
5.68 (br s, 2H), 4.64 (d, $J=14.4 \mathrm{~Hz}, 1 \mathrm{H}), 4.40$ (d, $J=14.4 \mathrm{~Hz}, 1 \mathrm{H}), 4.19$ (s, 2H), 3.78 (s, 3H), 
$3.28-3.12(\mathrm{~m}, 2 \mathrm{H}), 2.86(\mathrm{~d}, J=16.9 \mathrm{~Hz}, 1 \mathrm{H}), 2.52(\mathrm{~d}, J=16.9 \mathrm{~Hz}, 1 \mathrm{H}), 1.93-1.75(\mathrm{~m}, 3 \mathrm{H}), 1.73-$ $1.64(\mathrm{~m}, 1 \mathrm{H}), 0.90(\mathrm{~s}, 9 \mathrm{H}), 0.05(\mathrm{~s}, 6 \mathrm{H})$.

${ }^{13}$ C NMR (101MHz, $\left.\mathbf{C D C l}_{3}\right): \delta$ 200.7, 171.3, 158.9, 132.3, 131.1, 129.4, 129.2, 113.9, 63.1, 55.1, $52.3,49.8,47.2,46.3,32.3,25.8,18.8,18.3,-5.3$.

HRMS (ESI): Calculated for $[\mathrm{M}+\mathrm{Na}]^{+} \mathrm{C}_{24} \mathrm{H}_{37} \mathrm{NO} 4 \mathrm{SiNa}: 454.2390$, found : 454.2389.

(R,E)-2-(3-(3-((tert-butyldimethylsilyl)oxy)prop-1-en-1-yl)-1-(4-methoxy benzyl)-2oxopiperidin-3-yl)acetaldehyde:

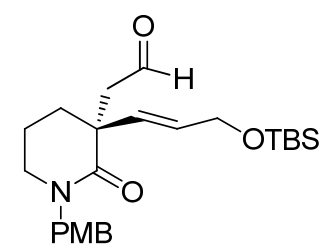

Yield: (0.7 g, $77 \%)$, colorless liquid.

$[\boldsymbol{\alpha}] \mathbf{D}^{\mathbf{2 4}}=-3.31\left(c=0.8, \mathrm{CHCl}_{3}\right)$.

${ }^{1} \mathbf{H}$ NMR (400MHz ,CDCl 3$): \delta 9.76(\mathrm{~s} ., 1 \mathrm{H}), 7.20$ (d, $\left.J=7.8 \mathrm{~Hz}, 2 \mathrm{H}\right), 6.85(\mathrm{~d}, J=7.6 \mathrm{~Hz}, 2 \mathrm{H})$, 5.69 (br s, 2H), 4.64 (d, $J=14.4 \mathrm{~Hz}, 1 \mathrm{H}), 4.41$ (d, $J=14.4 \mathrm{~Hz}, 1 \mathrm{H}), 4.19$ (s, 2H), 3.79 (s, 3H), $3.30-3.14(\mathrm{~m}, 2 \mathrm{H}), 2.87(\mathrm{~d}, J=16.9 \mathrm{~Hz}, 1 \mathrm{H}), 2.52(\mathrm{~d}, J=16.6 \mathrm{~Hz}, 1 \mathrm{H}), 1.94-1.79(\mathrm{~m}, 3 \mathrm{H}), 1.74-$ $1.64(\mathrm{~m}, 1 \mathrm{H}), 0.90(\mathrm{~s}, 9 \mathrm{H}), 0.06(\mathrm{~s}, 6 \mathrm{H})$.

HRMS (ESI): Calculated for [M + H] ${ }^{+} \mathrm{C}_{24} \mathrm{H}_{38} \mathrm{NO}_{4} \mathrm{Si}: 432.2570$, found :432.2573.

\section{Protection Of aldehyde as dithiane:}

To a solution of above mentioned aldehyde $(1 \mathrm{~g}, 2.32 \mathrm{mmol})$ in anhydrous $\mathrm{CH}_{2} \mathrm{Cl}_{2}(20 \mathrm{~mL}), 1,3-$ propanedithiol $(0.3 \mathrm{~mL}, 0.3 \mathrm{mmol})$ and boron trifluoride etherate $(1.14 \mathrm{~mL}, 9.27 \mathrm{mmol})$ were added and the reaction mixture was stirred at room temperature for $12 \mathrm{~h}$. The reaction mixture was quenched with saturated $\mathrm{NaHCO}_{3}(15 \mathrm{~mL})$ and the aqueous layer was extracted with dichloromethane. The combined organic layers were dried over $\mathrm{Na}_{2} \mathrm{SO}_{4}$ and concentrated. The crude reaction mixture was purified on neutral silica gel using ethyl acetate: hexane $(1: 1 \rightarrow 1: 0$ gradient) to afford dithiane as viscous liquid. 
(S,E)-3-((1,3-dithian-2-yl)methyl)-3-(3-hydroxyprop-1-en-1-yl)-1-(4-

methoxybenzyl)piperidin-2-one:

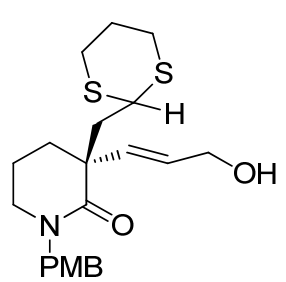

TLC: $\mathrm{R}_{f}=\mathbf{0 . 5}\left(\mathrm{SiO}_{2}\right.$, ethyl acetate: hexane $\left.=7: 3\right)$.

Yield: $(0.77 \mathrm{~g}, 82 \%)$, pale yellow color liquid.

$[\alpha]_{\mathbf{D}}^{24}=-1.69\left(c=0.64, \mathrm{CHCl}_{3}\right)$.

IR (film): 3410, 2932, 2860, 1614, 1511, $1245 \mathrm{~cm}^{-1}$.

${ }^{1}$ H NMR (400MHz , $\left.\mathbf{C D C l}_{3}\right): \delta 7.20(\mathrm{~d}, J=8.3 \mathrm{~Hz}, 2 \mathrm{H}), 6.84(\mathrm{~d}, J=8.6 \mathrm{~Hz}, 2 \mathrm{H}), 5.92$ - 5.68 (m, 2H), 4.50 (s, 2H), 4.15 (br s., 2H), 4.12 - 3.98 (m, 1H), 3.79 (s, 3H), 3.37 - 3.07 (m, 2H), 2.95 2.66 (m, 4H), 2.43 (dd, $J=7.3,14.6 \mathrm{~Hz}, 1 \mathrm{H}), 2.20$ - 1.97 (m, 3H), 1.95-1.82 (m, 4H), 1.70 (br s, $1 \mathrm{H})$.

${ }^{13} \mathbf{C}$ NMR (101MHz ,CDCl 3$): ~ \delta 171.5,158.8,135.6,129.6,129.5,129.4,113.8,63.4,55.2,50.1$, $47.9,47.3,44.3,42.9,30.3,30.2,30.1,25.3,19.2$.

HRMS (ESI): Calculated for $[\mathrm{M}+\mathrm{Na}]^{+} \mathrm{C}_{21} \mathrm{H}_{29} \mathrm{NNaO}_{3} \mathrm{~S}_{2}: 430.1487$, found : 430.1478 .

(R,E)-3-((1,3-dithian-2-yl)methyl)-3-(3-hydroxyprop-1-en-1-yl)-1-(4methoxybenzyl)piperidin-2-one:

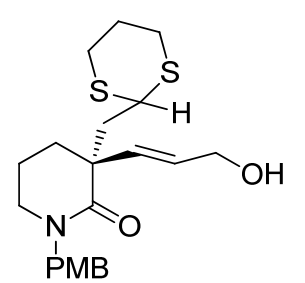

Yield: $(0.79 \mathrm{~g}, 84 \%)$, pale yellow color liquid.

$[\alpha] \mathbf{D}^{24}=+1.92\left(c=0.79, \mathrm{CHCl}_{3}\right)$. 
${ }^{1} \mathrm{H}$ NMR (400MHz, $\left.\mathbf{C D C l}_{3}\right): \delta 7.17(\mathrm{~d}, J=8.2 \mathrm{~Hz}, 2 \mathrm{H}), 6.82(\mathrm{~d}, J=8.2 \mathrm{~Hz}, 2 \mathrm{H}), 5.74$ (br s, 2H), 4.51 - $4.44(\mathrm{~m}, 2 \mathrm{H}), 4.15-4.10(\mathrm{~m}, 2 \mathrm{H}), 4.11-3.98(\mathrm{~m}, 1 \mathrm{H}), 3.76(\mathrm{~s}, 3 \mathrm{H}), 3.22-3.11(\mathrm{~m}, 2 \mathrm{H})$, $2.85-2.75(\mathrm{~m}, 4 \mathrm{H}), 2.58$ (br s, 1H), 2.42 (dd, $J=7.5,14.5 \mathrm{~Hz}, 1 \mathrm{H}), 2.06$ - 1.97 (m, 3H), 1.83 (m, $3 \mathrm{H}), 1.70(\mathrm{~m}, 1 \mathrm{H})$.

${ }^{13}$ C NMR (101MHz, $\left.\mathbf{C D C l}_{3}\right): \delta 171.5,158.7,135.3,129.6,129.5,129.3,113.7,63.1,55.1,50.1$, $47.8,47.3,44.1,42.8,30.2,30.1,30.0,25.2,19.1$.

HRMS (ESI): Calculated for $[\mathrm{M}+\mathrm{H}]^{+} \mathrm{C}_{21} \mathrm{H}_{30} \mathrm{NO}_{3} \mathrm{~S}_{2}: 408.1667$, found : 408.1658 .

\section{Reduction of dithiane:}

Raney Ni (3 g, W-2) was added to a solution of aldehyde (0.77 g, $1.89 \mathrm{mmol})$ in absolute ethanol $(15 \mathrm{~mL})$. The reaction mixture was refluxed for $16 \mathrm{~h}$ under hydrogen atmosphere $(1 \mathrm{~atm})$. The reaction mixture was filtered through celite, washed with ethyl acetate. The combined filtrate was concentrated and purified by column chromatography using ethyl acetate and hexane (4:6) mixture to obtain alcohol $\mathbf{1 5}$ as colorless liquid.

(R)-3-ethyl-3-(3-hydroxypropyl)-1-(4-methoxybenzyl)piperidin-2-one (15b) :

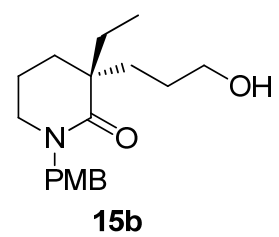

Yield: (490 mg, 85\%); colorless liquid.

TLC: $\mathrm{R}_{f}=\mathbf{0 . 4}\left(\mathrm{SiO}_{2}\right.$, ethyl acetate: hexane= 7:3 $)$.

$[\boldsymbol{\alpha}]_{\mathbf{D}}{ }^{\mathbf{2 3}}=-9.319\left(c=0.91, \mathrm{CHCl}_{3}\right)$.

IR (film): 3403, 2942, 2873, 1612, 1512, 1460, $1246 \mathrm{~cm}^{-1}$.

${ }^{1} \mathbf{H}$ NMR (800MHz ,CDCl 3$): \delta 7.18(\mathrm{~d}, J=8.6 \mathrm{~Hz}, 2 \mathrm{H}), 6.85(\mathrm{~d}, J=8.6 \mathrm{~Hz}, 2 \mathrm{H}), 4.58(\mathrm{~d}, J=$ $14.5 \mathrm{~Hz}, 1 \mathrm{H}), 4.43(\mathrm{~d}, J=14.5 \mathrm{~Hz}, 1 \mathrm{H}), 3.79$ (s, $3 \mathrm{H}), 3.63-3.56(\mathrm{~m}, 2 \mathrm{H}), 3.17$ (t, $J=6.1 \mathrm{~Hz}, 2$ H), 2.35 (br s, $1 \mathrm{H}), 1.88$ - $1.84(\mathrm{~m}, 1 \mathrm{H}), 1.79$ - 1.69 (m, $5 \mathrm{H}), 1.66$ - $1.62(\mathrm{~m}, 1 \mathrm{H}), 1.59-1.54$ (m, $1 \mathrm{H}), 1.51-1.47$ (m, $2 \mathrm{H}), 0.87$ (t, $J=7.4 \mathrm{~Hz}, 3 \mathrm{H})$. 
${ }^{13}$ C NMR (101MHz, $\left.\mathbf{C D C l}_{3}\right): \delta 175.1,158.7,129.6,129.3,113.8,62.7,55.1,49.8,47.4,44.7$, $34.2,31.7,28.9,27.7,19.6,8.6$.

HRMS (ESI): Calculated for $[\mathrm{M}+\mathrm{H}]^{+} \mathrm{C}_{18} \mathrm{H}_{28} \mathrm{NO}_{3}: 306.2069$, found : 306.2066 .

Synthesis of (S)-3-ethyl-3-(3-hydroxypropyl)-1-(4-methoxybenzyl)piperidin-2-one (15a):

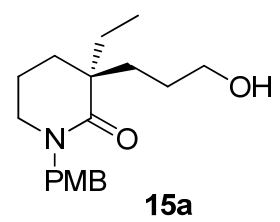

Yield: (325 mg, 85\%), colorless liquid.

$[\boldsymbol{\alpha}]_{\mathbf{D}}^{\mathbf{2 3}}=+10.216(c=0.8, \mathrm{CHCl} 3)$.

${ }^{1}$ H NMR (400MHz, $\left.\mathbf{C D C l}_{3}\right): \delta 7.17(\mathrm{~d}, J=8.6 \mathrm{~Hz}, 2 \mathrm{H}), 6.84$ (d, $\left.J=8.6 \mathrm{~Hz}, 2 \mathrm{H}\right), 4.58$ (d, $J=14.4$ $\mathrm{Hz}, 1 \mathrm{H}), 4.42(\mathrm{~d}, J=14.1 \mathrm{~Hz}, 1 \mathrm{H}), 3.79(\mathrm{~s}, 3 \mathrm{H}), 3.68-3.52$ (m, 2H), 3.17 (t, J=5.7 Hz, 2H), 2.36 (br s, 1H), $1.9-1.46(\mathrm{~m}, 10 \mathrm{H}), 0.87(\mathrm{t}, J=7.4 \mathrm{~Hz}, 3 \mathrm{H})$.

${ }^{13}$ C NMR (101MHz, $\left.\mathbf{C D C l}_{3}\right): \delta 175.1,158.8,129.6,129.4,113.9,62.8,55.2,49.9,47.4,44.7$, $34.1,31.8,28.9,27.7,19.7,8.6$.

HRMS (ESI): Calculated for $[\mathrm{M}+\mathrm{H}]^{+} \mathrm{C}_{18} \mathrm{H}_{28} \mathrm{NO}_{3}: 306.2069$, found : 306.2060 .

\section{Protection of primary alcohol as-OTBS ether:}

To a solution of compound $\mathbf{1 5 b}(1 \mathrm{~g}, 3.27 \mathrm{mmol})$ in dichloromethane $(25 \mathrm{~mL})$, imidazole $(0.165$ $\mathrm{g}, 7.15 \mathrm{mmol})$ and $\mathrm{TBSCl}(0.543 \mathrm{~g}, 3.6 \mathrm{mmol})$ were added at $0{ }^{\circ} \mathrm{C}$. After stirring for $1 \mathrm{~h}$, the reaction mixture was quenched with water. The aqueous layer was extracted with DCM $(3 \times 20 \mathrm{~mL})$ and the combined organic layer was dried over $\mathrm{Na}_{2} \mathrm{SO}_{4}$, concentrated and purified by column chromatography to afford corresponding silyl ether.

(R)-3-(3-((tert-butyldimethylsilyl)oxy)propyl)-3-ethyl-1-(4-methoxybenzyl) piperidin-2-one:<smiles>CC[C@@]1(CCCO[SbH3])CCCN([Y6](=O)[O-])C1=O</smiles> 
Yield: $1.37 \mathrm{~g}$, quantitative; colorless liquid.

TLC: $\mathrm{R}_{f}=\mathbf{0 . 5 5}\left(\mathrm{SiO}_{2}\right.$, ethyl acetate: hexane $=1.5: 8.5$, iodine/PMA, UV), as colorless liquid.

$[\boldsymbol{\alpha}]_{\mathbf{D}}{ }^{23}=+6.62\left(c=1.58, \mathrm{CHCl}_{3}\right)$.

IR (film): 2952, 2932, 2857, 1633, 1512, 1463, 1352, $1248 \mathrm{~cm}^{-1}$.

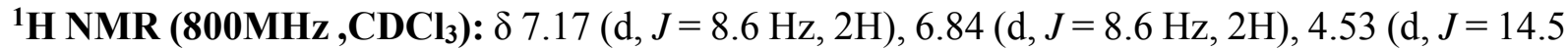
$\mathrm{Hz}, 1 \mathrm{H}), 4.48(\mathrm{~d}, J=14.5 \mathrm{~Hz}, 1 \mathrm{H}), 3.79(\mathrm{~s}, 3 \mathrm{H}), 3.65-3.52(\mathrm{~m}, 2 \mathrm{H}), 3.16(\mathrm{t}, J=5.9 \mathrm{~Hz}, 2 \mathrm{H})$, $1.83-1.76(\mathrm{~m}, 1 \mathrm{H}), 1.75-1.66(\mathrm{~m}, 5 \mathrm{H}), 1.59-1.44(\mathrm{~m}, 4 \mathrm{H}), 0.91-0.88(\mathrm{~m}, 9 \mathrm{H}), 0.87(\mathrm{t}, J=7.6$ $\mathrm{Hz}, 3 \mathrm{H}), 0.05(\mathrm{~s}, 6 \mathrm{H})$.

${ }^{13}$ C NMR (101MHz, $\left.\mathbf{C D C l}_{3}\right): \delta$ 174.7, 158.7, 129.9, 129.3, 113.8, 63.6, 55.1, 49.8, 47.4, 44.7, $34.7,31.5,29.1,27.8,25.9,19.8,18.3,8.7,-5.3$.

HRMS (ESI): Calculated for $[\mathrm{M}+\mathrm{H}]^{+} \mathrm{C}_{24} \mathrm{H}_{42} \mathrm{NO}_{3} \mathrm{Si}: 420.2934$, found : 420.2931 .

(S)-3-(3-((tert-butyldimethylsilyl)oxy)propyl)-3-ethyl-1-(4-methoxybenzyl) piperidin-2-one:

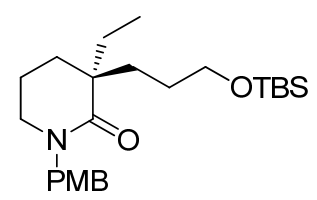

Yield: (1.37 g, quantitative), colorless liquid.

$[\boldsymbol{\alpha}] \mathbf{D}^{23}=-5.7\left(c=0.8, \mathrm{CHCl}_{3}\right)$

${ }^{1} \mathbf{H}$ NMR (400MHz ,CDCl $): \delta 7.17(\mathrm{~d}, J=8.3 \mathrm{~Hz}, 2 \mathrm{H}), 6.83(\mathrm{~d}, 2 \mathrm{H}), 4.63-4.38(\mathrm{~m}, 2 \mathrm{H}), 3.79$ $(\mathrm{s}, 3 \mathrm{H}), 3.68-3.45(\mathrm{~m}, 2 \mathrm{H}), 3.15(\mathrm{t}, J=5.5 \mathrm{~Hz}, 2 \mathrm{H}), 1.89-1.62(\mathrm{~m}, 6 \mathrm{H}), 1.62-1.39(\mathrm{~m}, 4 \mathrm{H})$, $1.04-0.78(\mathrm{~m}, 12 \mathrm{H}), 0.17-0.01(\mathrm{~m}, 6 \mathrm{H})$.

${ }^{13}$ C NMR (101MHz, $\left.\mathbf{C D C l}_{3}\right): \delta$ 174.7, 158.7, 129.9, 129.4, 129.3, 113.9, 113.8, 77.3, 76.7, 63.7, $55.2,49.8,47.4,44.8,34.7,31.5,29.1,27.8,25.9,19.8,18.3,8.8,-5.3$.

HRMS (ESI): Calculated for $[\mathrm{M}+\mathrm{H}]^{+} \mathrm{C}_{24} \mathrm{H}_{42} \mathrm{NO}_{3} \mathrm{Si}: 420.2934$, found : 420.2929. 


\section{$N$-PMB deprotection:}

A solution of $N$-PMB lactam $(1 \mathrm{~g}, 2.38 \mathrm{mmol})$ in anhydrous THF $(15 \mathrm{~mL})$ was charged into $100 \mathrm{~mL}$ two neck round bottom flask equipped with a magnetic stirring bar, cold finger condenser and argon balloon was cooled to $-78{ }^{\circ} \mathrm{C}$. Ammonia gas was condensed (through $\mathrm{KOH}$ column) to the reaction flask at $-78{ }^{\circ} \mathrm{C}$ from ammonia cylinder, and a small sodium piece was added to the stirred mixture till blue coloration persist. The resulting blue color solution was warmed to $-33{ }^{\circ} \mathrm{C}$ and stirring was continued for $1 \mathrm{~h}$ (temperature in the cold finger condenser was maintained $-78{ }^{\circ} \mathrm{C}$ till reaction completed). The reaction mixture was quenched by adding solid ammonium chloride in small portions till blue coloration disappeared, ammonia in the reaction mixture was evaporated at room temperature for overnight. The reaction mixture was filtered and the solid residues were washed with ethyl acetate. The filtrate was concentrated and purified by column chromatography to obtained $\mathbf{1 6 .}$

(R)-3-(3-((tert-butyldimethylsilyl)oxy)propyl)-3-ethylpiperidin-2-one (16b):

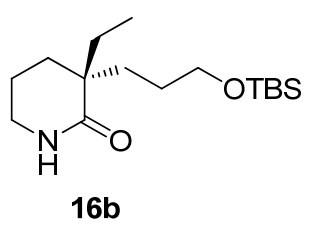

Yield: $(0.61 \mathrm{~g}, 85 \%)$; colorless liquid.

TLC: $\mathrm{R}_{f}=\mathbf{0 . 4}\left(\mathrm{SiO}_{2}\right.$, ethyl acetate: hexane $=6: 4$, iodine/PMA $)$.

$[\boldsymbol{\alpha}]_{\mathbf{D}} \mathbf{2 4}^{24}+19.67\left(c=0.36, \mathrm{CHCl}_{3}\right)$.

IR (film): 3289, 3212, 2951, 2858, 1658, 1469, $1254 \mathrm{~cm}^{-1}$.

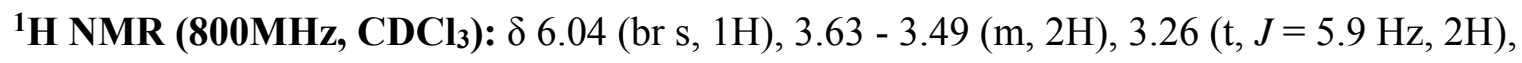
$1.81-1.76(\mathrm{~m}, 2 \mathrm{H}), 1.75-1.62(\mathrm{~m}, 4 \mathrm{H}), 1.57-1.44(\mathrm{~m}, 4 \mathrm{H}), 0.89-0.86(\mathrm{~m}, 12 \mathrm{H}), 0.03(\mathrm{~s}, 6 \mathrm{H})$.

${ }^{13}$ C NMR (101MHz, $\left.\mathbf{C D C l}_{3}\right): \delta 177.2,63.6,44.5,42.6,34.3,31.0,29.0,27.7,25.9,19.8,18.3$, 8.6, -5.3.

HRMS (ESI): Calculated for [M + H] ${ }^{+} \mathrm{C}_{16} \mathrm{H}_{34} \mathrm{NO}_{2} \mathrm{Si}: 300.2359$, found: 300.2349 . 
(S)-3-(3-((tert-butyldimethylsilyl)oxy)propyl)-3-ethylpiperidin-2-one (16a):

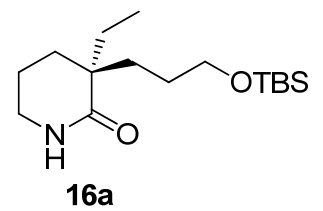

Yield: $(0.62 \mathrm{~g}, 87 \%)$, colorless liquid.

$[\boldsymbol{\alpha}]_{\mathbf{D}}{ }^{24}=-20.442\left(c=0.80, \mathrm{CHCl}_{3}\right)$.

${ }^{1}$ H NMR (400 MHz, CDCl $): \delta 6.17$ (br s, 1H), 3.62 - 3.51 (m, 2H), 3.25 (t, $\left.J=5.4 \mathrm{~Hz}, 2 \mathrm{H}\right)$, $1.83-1.61(\mathrm{~m}, 6 \mathrm{H}), 1.58-1.43(\mathrm{~m}, 4 \mathrm{H}), 0.92-0.82(\mathrm{~m}, 12 \mathrm{H}), 0.03(\mathrm{~s}, 6 \mathrm{H})$.

${ }^{13}$ C NMR (101MHz, $\left.\mathbf{C D C l}_{3}\right): \delta$ 177.3, 63.6, 44.4, 42.6, 34.3, 31.0, 29.0, 27.7, 25.9, 19.8, 18.3, 8.6, -5.3.

HRMS (ESI): Calculated for $[\mathrm{M}+\mathrm{H}]^{+} \mathrm{C}_{16} \mathrm{H}_{34} \mathrm{NO}_{2} \mathrm{Si}: 300.2359$, found: 300.2350 .

\section{$N$-Boc Protection:}

(R)-tert-butyl 3-(3-((tert-butyldimethylsilyl)oxy)propyl)-3-ethyl-2-oxopiperidine-1carboxylate:

To a Solution of above mentioned lactam $(1 \mathrm{~g}, 3.34 \mathrm{mmol})$ in dry THF, LiHMDS solution (1M in THF, $3.67 \mathrm{~mL}, 3.67 \mathrm{mmol}$ ) was added at $-78^{\circ} \mathrm{C}$. After stirring for $30 \mathrm{~min}$, di-tert-butyl dicarbonate $(0.85 \mathrm{~mL}, 3.67 \mathrm{mmol})$ was added. The reaction temperature was brought to room temperature and stirred for $2 \mathrm{~h}$ before being quenched with saturated aqueous $\mathrm{NH}_{4} \mathrm{Cl}$ Solution $(15 \mathrm{~mL})$. The aqueous layer was separated and extracted with ethyl acetate $(3 \times 15 \mathrm{~mL})$. The combined organic layers were dried over $\mathrm{Na}_{2} \mathrm{SO}_{4}$ and concentrated under reduced pressure and purified by column chromatography using ethyl acetate and hexane (0.5:9.5) mixture to afforded $N$-Boc-Lactam.

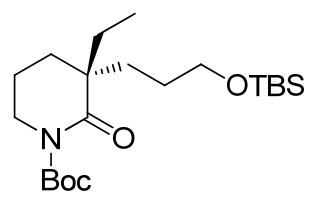


Yield: (1.3 g, 97\%), colorless liquid.

TLC: $\mathrm{R}_{f}=\mathbf{0 . 5}\left(\mathrm{SiO}_{2}\right.$, ethyl acetate: hexane $\left.=1: 9\right)$.

$[\alpha]_{\mathbf{D}}^{24}=-2.53\left(c=1.55, \mathrm{CHCl}_{3}\right)$.

IR (film): 2954, 2933, 2884, 2858, 1766, 1716, 1462, 1389, 1367, 1278, 1298, $1254 \mathrm{~cm}^{-1}$.

${ }^{1}$ H NMR (800MHz, CDCl$)$ ): $\delta 3.62$ - $3.52(\mathrm{~m}, 4 \mathrm{H}), 1.84$ - $1.77(\mathrm{~m}, 2 \mathrm{H}), 1.75$ - $1.66(\mathrm{~m}, 3 \mathrm{H})$,

$1.65-1.52(\mathrm{~m}, 3 \mathrm{H}), 1.51-1.43(\mathrm{~m}, 11 \mathrm{H}), 0.87(\mathrm{~s}, 9 \mathrm{H}), 0.85(\mathrm{t}, J=7.5 \mathrm{~Hz}, 3 \mathrm{H}), 0.02(\mathrm{~s}, 6 \mathrm{H})$.

${ }^{13}$ C NMR (101MHz, $\left.\mathbf{C D C l}_{3}\right): \delta 177.0,153.8,82.2,63.4,47.8,47.1,33.9,30.8,30.6,28.0,27.4$, $25.9,20.1,18.3,8.4,-5.3$.

HRMS (ESI): Calculated for $[\mathrm{M}+\mathrm{Na}]^{+} \mathrm{C}_{21} \mathrm{H}_{41} \mathrm{NO}_{4} \mathrm{SiNa}$ : 422.2703, found : 422.2693 .

(S)-tert-butyl 3-(3-((tert-butyldimethylsilyl)oxy)propyl)-3-ethyl-2-oxopiperidine-1carboxylate:

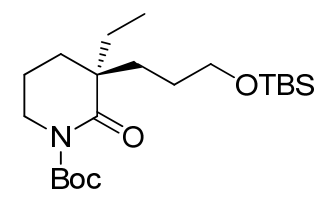

Yield: (1.26 g, $95 \%)$, colorless liquid.

$[\boldsymbol{\alpha}]_{\mathbf{D}}^{\mathbf{2 4}}=+3.12\left(c=1, \mathrm{CHCl}_{3}\right)$.

${ }^{1}$ H NMR (400MHz, CDCl$\left._{3}\right): \delta 3.65$ - 3.47 (m, 4H), 1.85 - $1.76(\mathrm{~m}, 2 \mathrm{H}), 1.75$ - 1.70 (m, 2H), $1.69-1.52(\mathrm{~m}, 4 \mathrm{H}), 1.52-1.45(\mathrm{~m}, 11 \mathrm{H}), 0.93-0.77(\mathrm{~m}, 12 \mathrm{H}), 0.08--0.06(\mathrm{~m}, 6 \mathrm{H})$.

${ }^{13}$ C NMR (101MHz, $\left.\mathbf{C D C l}_{3}\right): \delta 177.0,153.8,82.3,63.4,47.8,47.1,33.9,30.8,30.6,28.0,27.4$, $25.9,20.1,18.3,8.4,-5.3$.

HRMS (ESI): Calculated for [M + Na $]^{+} \mathrm{C}_{21} \mathrm{H}_{41} \mathrm{NO}_{4} \mathrm{SiNa}: 422.2703$, found : 422.2689 . 


\section{Deprotection of-OTBS ether:}

To a solution of 16 ( $0.3 \mathrm{~g}, 0.75 \mathrm{mmol})$ in methanol, $p$-toluene sulfonic acid (0.006 g, $0.04 \mathrm{mmol})$ was added at $-10^{\circ} \mathrm{C}$. The solution was stirred for $30 \mathrm{~min}$ and quenched with saturated aqueous $\mathrm{NaHCO}_{3}$ solution at $-10{ }^{\circ} \mathrm{C}$. The solution was evaporated to dryness under reduced pressure. The residue was dissolved in ethyl acetate and washed with saturated $\mathrm{NaCl}$ solution, separated the layers and concentrated, quick purification using Flash chromatography using ethyl acetate and hexane (3:7) mixture to provided 17.

(R)-tert-butyl 3-ethyl-3-(3-hydroxypropyl)-2-oxopiperidine-1-carboxylate (17b):

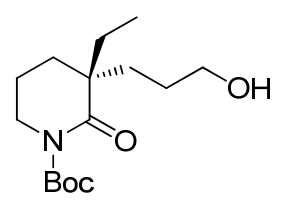

$17 b$

Yield: $(0.2$ g, 92\%), colorless liquid.

TLC: $\mathrm{R}_{f}=0.5\left(\mathrm{SiO}_{2}\right.$, ethylacetate $:$ hexane $\left.=4: 6\right)$.

$[\boldsymbol{\alpha}] \mathbf{D}^{25}=+3.76\left(c=0.5, \mathrm{CHCl}_{3}\right)$.

IR (film): 2935, 2877, 1760, 1714, 1458, 1391, 1368, 1297, 1277, $1254 \mathrm{~cm}^{-1}$.

${ }^{1}$ H NMR (800MHz, CDCl $)$ ): $\delta 3.68$ - $3.55(\mathrm{~m}, 4 \mathrm{H}), 1.87-1.80(\mathrm{~m}, 2 \mathrm{H}), 1.77-1.69(\mathrm{~m}, 4 \mathrm{H})$, $1.64-1.54$ (m, 4H), 1.51 (s, 9H), 0.88 (t, $J=7.4 \mathrm{~Hz}, 3 \mathrm{H})$.

${ }^{13}$ C NMR (101MHz, $\left.\mathbf{C D C l}_{3}\right): \delta 177.1,153.8,82.5,63.0,47.8,47.2,33.7,31.1,30.6,28.0,27.5$, 20.1, 8.4.

HRMS (ESI): Calculated for $[\mathrm{M}+\mathrm{Na}]^{+} \mathrm{C}_{15} \mathrm{H}_{27} \mathrm{NO}_{4} \mathrm{Na}: 308.1838$, found :308.1832.

(S)-tert-butyl 3-ethyl-3-(3-hydroxypropyl)-2-oxopiperidine-1-carboxylate (17a):

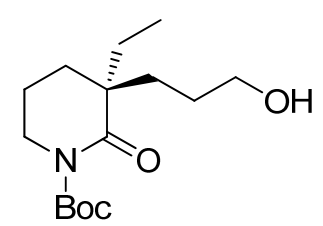

$17 a$ 
Yield: $(0.2 \mathrm{~g}, 93 \%)$, colorless liquid.

$[\boldsymbol{\alpha}]_{\mathbf{D}}{ }^{24}=-3.25\left(\mathrm{CHCl}_{3}, c=0.35\right)$.

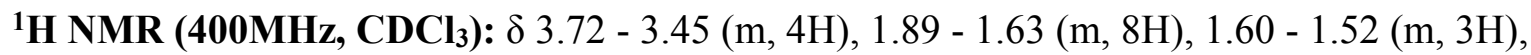
$1.50(\mathrm{~s}, 9 \mathrm{H}), 0.87$ (t, $J=7.48 \mathrm{~Hz}, 3 \mathrm{H})$.

${ }^{13}$ C NMR (101MHz, $\left.\mathbf{C D C l}_{3}\right): \delta 177.1,153.7,82.5,62.9,47.7,47.2,33.6,31.1,30.5,28.0,27.4$, $20.0,8.4$.

HRMS (ESI): Calculated for $[\mathrm{M}+\mathrm{Na}]^{+} \mathrm{C}_{15} \mathrm{H}_{27} \mathrm{NO}_{4} \mathrm{Na}$ : 308.1838 , found : 308.1834 .

\section{Oxidation of alcohol:}

To a solution of alcohol $17(1 \mathrm{~g}, 3.5 \mathrm{mmol})$ in anhydrous dichloromethane (30 mL), Dess-Martin periodinane $(1.78 \mathrm{~g}, 4.2 \mathrm{mmol})$ was added at $0{ }^{\circ} \mathrm{C}$. The reaction temperature was slowly raised to room temperature by removing ice tub and stirring was continued for $30 \mathrm{~min}$. The reaction was quenched successively with a saturated aqueous $\mathrm{Na}_{2} \mathrm{~S}_{2} \mathrm{O}_{3}$ solution $(5 \mathrm{ml})$ and saturated aqueous $\mathrm{NaHCO}_{3}$ solution $(10 \mathrm{ml})$, stirred for $30 \mathrm{~min}$. The aqueous layer was extracted with dichloromethane $(3 \times 30 \mathrm{~mL})$. The combined dichloromethane layer was dried over $\mathrm{Na}_{2} \mathrm{SO}_{4}$ and evaporated and residue was purified by column chromatography using ethyl acetate and hexane $(1: 9 \rightarrow 2: 8$ gradient $)$ to afford corresponding aldehyde.

\section{(R)-tert-butyl 3-ethyl-2-oxo-3-(3-oxopropyl)piperidine-1-carboxylate:}

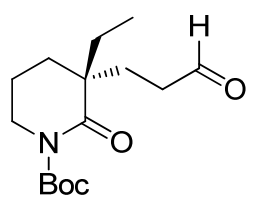

Yield: (0.95 g, 96\%); colorless liquid.

TLC: $\mathrm{R}_{f}=\mathbf{0 . 6}\left(\mathrm{SiO}_{2}\right.$, ethyl acetate: hexane $=3: 7$, iodine $\left./ \mathrm{PMA}\right)$.

$[\boldsymbol{\alpha}] \mathbf{D}^{23}=+2.13\left(c=0.5, \mathrm{CHCl}_{3}\right)$

IR (neat): 3206, 2974, 2940, 1759, 1712, 1456, 1392, 1280, $1297 \mathrm{~cm}^{-1}$. 
${ }^{1} \mathbf{H}$ NMR (800MHz, CDCl$): \delta 9.77(\mathrm{~s}, 1 \mathrm{H}), 3.61(\mathrm{t}, J=5.9 \mathrm{~Hz}, 2 \mathrm{H}), 2.51(\mathrm{t}, J=7.8 \mathrm{~Hz}, 2 \mathrm{H}), 1.98$ - $1.62(\mathrm{~m}, 8 \mathrm{H}), 1.51(\mathrm{~s}, 9 \mathrm{H}), 0.89(\mathrm{t}, J=7.4 \mathrm{~Hz}, 3 \mathrm{H})$.

${ }^{13}$ C NMR (201MHz, $\left.\mathbf{C D C l}_{3}\right): \delta 201.9,176.3,153.6,82.6,47.2,39.3,30.9,30.4,29.2,27.9,19.8$, 8.2 .

HRMS (ESI): Calculated for $[\mathrm{M}+\mathrm{Na}]^{+} \mathrm{C}_{15} \mathrm{H}_{25} \mathrm{NO}_{4} \mathrm{Na}: 306.1681$, found : 306.1676.

(S)-tert-butyl 3-ethyl-2-oxo-3-(3-oxopropyl)piperidine-1-carboxylate:

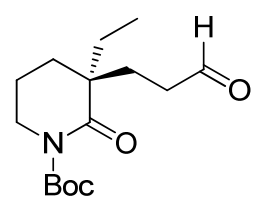

Yield: $(0.96 \mathrm{~g}, 97 \%)$, colorless liquid.

$[\boldsymbol{\alpha}]_{\mathbf{D}}^{24}=-2.72\left(\mathrm{CHCl}_{3}, c=0.8\right)$.

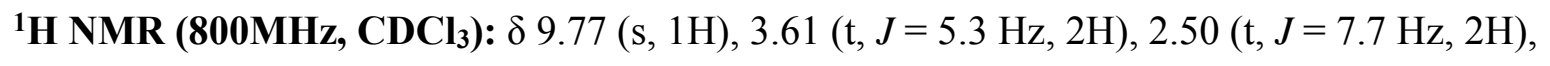
$1.96-1.61(\mathrm{~m}, 8 \mathrm{H}), 1.50(\mathrm{~m}, 9 \mathrm{H}), 0.88(\mathrm{t}, J=7.3 \mathrm{~Hz}, 3 \mathrm{H})$.

${ }^{13}$ C NMR (101MHz, $\left.\mathbf{C D C l}_{3}\right): \delta$ 201.9, 176.3, 153.6, 82.6, 47.1, 39.3, 30.9, 30.4, 29.2, 28.0, 19.9, 8.2.

HRMS (ESI): Calculated for $[\mathrm{M}+\mathrm{Na}]^{+} \mathrm{C}_{15} \mathrm{H}_{25} \mathrm{NO}_{4} \mathrm{Na}: 306.1681$, found : 306.1674 .

Vinylation of aldehyde:

To a stirred solution of aldehyde $(0.5 \mathrm{~g}, 1.76 \mathrm{mmol})$ in anhydrous ether, vinyl magnisium bromide $(2.12 \mathrm{~mL}, 2.12 \mathrm{mmol})$ was added at $-78{ }^{\circ} \mathrm{C}$. After $6 \mathrm{~h}$ stirring at $-78{ }^{\circ} \mathrm{C}$, it was quenched with saturated aqueous ammonium chloride solution and warmed to room temperature. The solution was extracted with ethyl acetate and concentrated. The crude residue was purified by flash column chromatography using ethyl acetate and hexane (1.5:8.5) mixture to obtain allyl alcohol 18 as pale yellow color liquid. 
(3R)-tert-butyl 3-ethyl-3-(3-hydroxypent-4-en-1-yl)-2-oxopiperidine-1-carboxylate (18b):

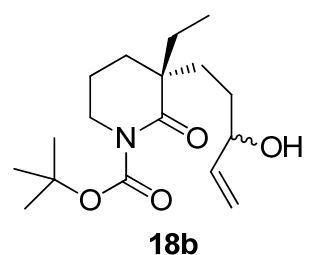

Yield: (0.42 g, 75\%); pale yellow color liquid.

TLC: $\mathrm{R}_{f}=\mathbf{0 . 5}\left(\mathrm{SiO}_{2}\right.$, ethyl acetate: hexane= 3:7).

IR (neat): 3379, 2971, 2939, 2879, 1761, 1712, 1522, 1459, 1367, 1275, $1252 \mathrm{~cm}^{-1}$.

${ }^{1} \mathbf{H}$ NMR (400MHz ,CDCl$\left.)_{3}\right)$ : mixture of two diasteromers $\delta 5.92$ - $5.77(\mathrm{~m}, 1 \mathrm{H})$, 5.38-5.06 (m, 2H), 4.84 - $4.72(\mathrm{~m}, 0.5 \mathrm{H}), 4.60$ (br s, 1H), 4.09 - $4.01(\mathrm{~m}, 0.5 \mathrm{H}), 3.65$ - $3.53(\mathrm{~m}, 1 \mathrm{H}), 3.08$ (d, $J$ $=5.8 \mathrm{~Hz}, 1 \mathrm{H}), 2.00-1.90(\mathrm{~m}, 1 \mathrm{H}), 1.84-1.54(\mathrm{~m}, 9 \mathrm{H}), 1.49(\mathrm{~s}, 4.5 \mathrm{H}), 1.42(\mathrm{~s}, 4.5 \mathrm{H}), 0.93-0.84$ (m, 3H).

${ }^{13} \mathbf{C}$ NMR (101MHz, $\left.\mathbf{C D C l}_{3}\right):$ mixture of two diasteromers $\delta$ 177.0, 175.6, 156.0, 153.7, 140.9, $136.4,116.7,114.6,82.4,80.5,73.1,47.8,47.1,45.3,36.1,33.2,31.8,31.5,31.1,30.6,28.4,28.0$, $27.8,26.5,25.0,20.1,8.6,8.3$.

HRMS (ESI): Calculated for [M + Na] $]^{+} \mathrm{C}_{17} \mathrm{H}_{29} \mathrm{NO}_{4} \mathrm{Na}$ : 334.1994, found: 334.1990.

(3S)-tert-butyl 3-ethyl-3-(3-hydroxypent-4-en-1-yl)-2-oxopiperidine-1-carboxylate (18a):

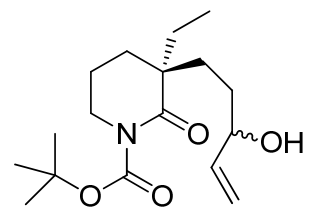

$18 \mathbf{a}$

Yield: $(0.34 \mathrm{~g}, 77 \%)$, pale yellow color liquid.

${ }^{1} \mathbf{H}$ NMR (800MHz, $\left.\mathbf{C D C l}_{3}\right)$ : mixture of two diasteromers $\delta 5.91-5.80(\mathrm{~m}, 1 \mathrm{H}), 5.39-5.03(\mathrm{~m}$, 2H), $4.79-4.76(\mathrm{~m} \mathrm{0.5H}), 4.60$ (br s, 1H), $4.10-4.00(\mathrm{~m}, 0.5 \mathrm{H}), 3.62$ - $3.55(\mathrm{~m}, 1 \mathrm{H}), 3.12$ - 3.06 $(\mathrm{m}, 1 \mathrm{H}), 1.84-1.56(\mathrm{~m}, 10 \mathrm{H}), 1.49(\mathrm{~s}, 4.5 \mathrm{H}), 1.43(\mathrm{~s}, 4.5 \mathrm{H}), 0.92-0.86(\mathrm{~m}, 3 \mathrm{H})$. 
${ }^{13} \mathbf{C}$ NMR (101 MHz, $\left.\mathbf{C D C l}_{3}\right)$ : mixture of two diasteromers $\delta 177.1,175.7,155.9,153.7,140.9$, $136.4,136.4,136.4,116.7,114.9,114.6,80.7,80.5,79.1$, 73.0, 48.4, 47.7, 47.1, 45.3, 40.7, 36.1, $35.3,33.2,31.8,28.3,27.9,27.8,26.5,26.3,25.0,20.1,8.6,8.3$.

HRMS (ESI): Calculated for $[\mathrm{M}+\mathrm{Na}]^{+} \mathrm{C}_{17} \mathrm{H}_{29} \mathrm{NO}{ }_{4} \mathrm{Na}: 334.1994$, found: 334.1987.

\section{$N$-Boc Bicyclic aminal synthesis:}

DIBAL-H (2.89 mmol, $2.89 \mathrm{~mL}, 1 \mathrm{M}$ solution in hexane) was added to a solution of compound $\mathbf{1 8 b}(0.3 \mathrm{~g}, 0.96 \mathrm{mmol})$ in dry THF $(10 \mathrm{~mL})$ at $-78^{\circ} \mathrm{C}$ and stirred for $2 \mathrm{~h}$. The reaction was quenched with saturated aqueous sodium potassium tartrate solution $(20 \mathrm{~mL})$ and warmed to room temperature. The reaction mixture was extracted with ethyl acetate $(3 \times 25 \mathrm{~mL})$, dried and concentrated to obtain crude hemiaminal as white foam.

The crude hemiaminal was dried under high vacuum pump and dissolved in dry dichloromethane, charged with pyridinium $p$-toluene sulphonate $(0.045 \mathrm{~g}, 0.192 \mathrm{mmol})$ at room temperature under Ar atmosphere, and stirred for $2 \mathrm{~h}$. The reaction mixture was quenched with a saturated aqueous $\mathrm{NaHCO}_{3}$ solution $(10 \mathrm{~mL})$. The aqueous layer was extracted with dichloromethane $(3 \times 25 \mathrm{ml})$. The combined organic layer was concentrated under reduced pressure and the crude was dissolved in hexane, filtered and concentrated. The crude product was dried under high vacuum which resulted pure 19b pale yellow color liquid $(0.27 \mathrm{~g})$. This crude was obtained as a mixture of two diasteromers, and the same was used for further reaction without any Column chromatography purification (small quantity was purified for taking NMR and other analytical data).

(4aR, 8aS)-tert-butyl 4a-ethyl-2-vinylhexahydro-2H-pyrano[2,3-b]pyridine-8(8aH)carboxylate (19b):

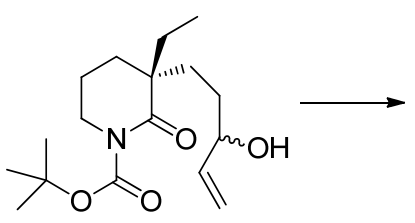

$18 b$

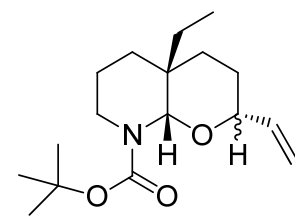

$19 b$ 
TLC: $\mathrm{R}_{f}=\mathbf{0 . 4 5}\left(\mathrm{SiO}_{2}\right.$, ethyl acetate: hexane $\left.=0.5: 9.5\right)$.

IR (neat): 3377, 2967, 2941, 2871, 1700, 1417, 1367, $1157 \mathrm{~cm}^{-1}$.

${ }^{1}$ H NMR (400MHz, $\left.\mathbf{C D C l}_{3}\right): \delta 6.01-5.76(\mathrm{~m}, 1 \mathrm{H}), 5.42$ - $5.20(\mathrm{~m}, 2 \mathrm{H}), 5.14-5.00(\mathrm{~m}, 1 \mathrm{H}), 4.58$ (m, 0.5H), $4.07-3.83(\mathrm{~m}, 1.5 \mathrm{H}), 3.15-2.88(\mathrm{~m}, 1 \mathrm{H}), 2.11-1.81(\mathrm{~m}, 2 \mathrm{H}), 1.73-1.47(\mathrm{~m}, 6 \mathrm{H})$, $1.47-1.41(\mathrm{~m}, 9 \mathrm{H}), 1.23-1.10(\mathrm{~m}, 2 \mathrm{H}), 0.86-0.69(\mathrm{~m}, 3 \mathrm{H})$.

${ }^{13} \mathbf{C}$ NMR (101MHz ,CDCl $\left.\mathbf{3}\right): \delta 155.1,138.7,137.4,116.7,114.8,114.5,86.8,85.6,79.9,79.6$, $77.8,77.7$, , 73.3, 39.3, 38.3, 38.2 , 34.5, 34.2, 33.1, 33.0, 28.5, 28.3, 27.8, 27.6, 27.5, 27.0, 26.9, $24.0,23.6,23.5,23.0,20.3,20.0,19.9,6.7,6.6,6.4$.

HRMS (ESI): Calculated for $[\mathrm{M}+\mathrm{Na}]^{+}: \mathrm{C}_{17} \mathrm{H}_{29} \mathrm{NO}_{3} \mathrm{Na}$ 318.2045, found: 318.2039 .

(4aS,8aR)-tert-butyl4a-ethyl-2-vinylhexahydro-2H-pyrano[2,3-b]pyridine-8(8aH)carboxylate (19a):

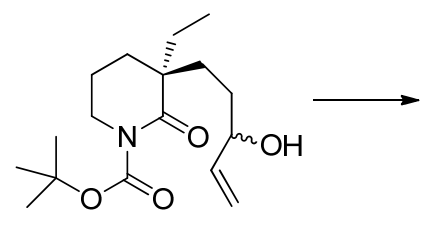

18a

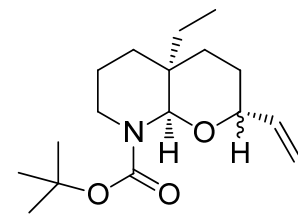

$19 a$

Yield: $0.21 \mathrm{~g}$, pale yellow color liquid.

${ }^{1}$ H NMR (400MHz, CDCl $)$ ): $\delta 5.94$ - $5.75(\mathrm{~m}, 1 \mathrm{H}), 5.41$ - $5.14(\mathrm{~m}, 2 \mathrm{H}), 5.11$ - $4.96(\mathrm{~m}, 1 \mathrm{H}), 4.61$ - $4.44(\mathrm{~m}, 0.5 \mathrm{H}), 3.94-3.78(\mathrm{~m}, 1.5 \mathrm{H}), 3.09$ - $2.90(\mathrm{~m}, 1 \mathrm{H}), 2.06-1.76(\mathrm{~m}, 2 \mathrm{H}), 1.68$ - $1.44(\mathrm{~m}$, 6H), 1.42 (s., 9H), $1.18-1.08$ (m , 2H), $0.78-0.70$ (m, 3H).

${ }^{13}$ C NMR (101MHz, $\left.\mathbf{C D C l}_{3}\right): \delta$ 155.1, 155.0, 138.7, 137.4, 116.6, 114.7, 114.5, 86.7, 85.5, 79.9, 79.6, 77.7, 77.6, 73.2, 39.3, 38.3, 38.1, 34.4, 34.2, 33.1, 32.9, 28.5, 28.3, 27.7, 27.6, 27.5, 26.9, $26.8,24.0,23.5,23.5,22.9,20.3,20.0,19.9,6.6,6.4$.

HRMS (ESI): Calculated for $[\mathrm{M}+\mathrm{Na}]^{+}: \mathrm{C}_{17} \mathrm{H}_{29} \mathrm{NNaO}_{3}: 318.2045$, found: 318.2036 .

\section{$N$-Boc deprotection:}

Triethylamine $(0.26 \mathrm{~mL}, 1.83 \mathrm{mmol})$ and TMSOTf $(0.25 \mathrm{~mL}, 1.37 \mathrm{mmol})$ were added to a stirred solution of compound $19(0.27 \mathrm{~g}, 0.914 \mathrm{mmol})$ in dry dichloromethane $(10 \mathrm{~mL})$ at $0{ }^{\circ} \mathrm{C}$. After $1 \mathrm{~h}$ stirring at room temperature, the reaction was quenched with saturated $\mathrm{NaHCO}_{3}$ solution. The 
mixture was extracted with Dichloromethane $(3 \times 25 \mathrm{~mL})$. The combined organic layer was dried over $\mathrm{Na}_{2} \mathrm{SO}_{4}$ and concentrated under reduced pressure. The crude compound was dissolved in hexane and filtered. The filtrate was concentrated by rotavapour and dried under moderate vacuum for 15 min which resulted pure bicyclic amine (small quantity was purified for taking NMR and other analytical data).

(4aR, 8aS)-4a-ethyl-2-vinyloctahydro-2H-pyrano[2,3-b]pyridine:

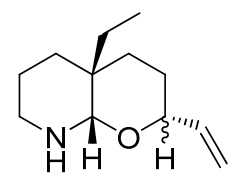

Yield: $0.17 \mathrm{~g}$, colorless liquid.

TLC: $\mathrm{R}_{f}=\mathbf{0 . 5}\left(\mathrm{SiO}_{2}\right.$, ethylacetate $:$ hexane $=3: 7$, Iodine/PMA), as colorless liquid.

IR (neat): $3341,2937,2861,1466,1445,1310 \mathrm{~cm}^{-1}$.

${ }^{1}$ H NMR (800MHz, CDCl $\left.{ }_{3}\right): \delta 5.97-5.84(\mathrm{~m}, 1 \mathrm{H}), 5.26(\mathrm{~d}, J=17.2 \mathrm{~Hz}, 0.5 \mathrm{H}), 5.21(\mathrm{~d}, J=17.6$ $\mathrm{Hz}, 0.5 \mathrm{H}), 5.13-5.08(\mathrm{~m}, 1 \mathrm{H}), 4.54-4.49(\mathrm{~m}, 0.5 \mathrm{H}), 4.11(\mathrm{~s}, 0.5 \mathrm{H}), 3.95(\mathrm{~s}, 0.5 \mathrm{H}), 3.85-3.79$ (m, 0.5H), 3.12 - $3.06(\mathrm{~m}, 1 \mathrm{H}), 2.67-2.61(\mathrm{~m}, 1 \mathrm{H}), 2.07$ (br s, 1H), 1.93 - $1.84(\mathrm{~m}, 1 \mathrm{H}), 1.78-1.72$ $(\mathrm{m}, 1 \mathrm{H}), 1.65-1.52(\mathrm{~m}, 3 \mathrm{H}), 1.47-1.22(\mathrm{~m}, 4 \mathrm{H}), 1.18$ - $1.12(\mathrm{~m}, 1 \mathrm{H}), 0.82-0.77(\mathrm{~m}, 3 \mathrm{H})$.

${ }^{13}$ C NMR (101MHz, CDCl $): \delta 139.4,139.3,115.2,114.5,90.0,70.7,39.3,34.1,33.8,33.4,28.9$, $28.8,27.4,26.1,24.5,21.1,20.9,6.9,6.8$.

HRMS (ESI): Calculated for [M $+\mathrm{H}]^{+} \mathrm{C}_{12} \mathrm{H}_{22} \mathrm{NO}$ : 196.1701, found: 196.1708 .

(4aS,8aR)-4a-ethyl-2-vinyloctahydro-2H-pyrano[2,3-b]pyridine :

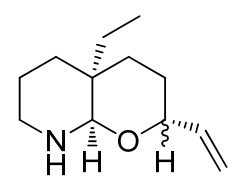

Yield: (0.136 g, 94\%), colorless liquid. 


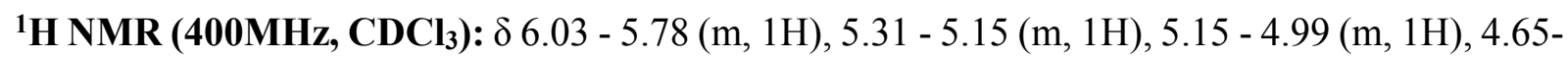
$4.41(\mathrm{~m}, 0.5 \mathrm{H}), 4.10(\mathrm{~s}, 0.5 \mathrm{H}), 3.94(\mathrm{~s}, 0.5 \mathrm{H}), 3.86-3.74(\mathrm{~m}, 0.5 \mathrm{H}), 3.17$ - $2.98(\mathrm{~m}, 1 \mathrm{H}), 2.74$ $2.54(\mathrm{~m}, 1 \mathrm{H}), 1.95-1.82(\mathrm{~m}, 2 \mathrm{H}), 1.78-1.22(\mathrm{~m}, 8 \mathrm{H}), 1.19-1.10(\mathrm{~m}, 1 \mathrm{H}), 0.84-0.73(\mathrm{~m}, 3 \mathrm{H})$.

${ }^{13}$ C NMR (101MHz, $\left.\mathbf{C D C l}_{3}\right): \delta 139.5,139.4,115.1,114.5,90.1,70.7,39.3,34.1,33.8,33.4,28.9$, $28.8,27.4,26.1,24.6,21.1,20.9,6.8,6.7$.

HRMS (ESI): Calculated for $[\mathrm{M}+\mathrm{H}]^{+} \mathrm{C}_{12} \mathrm{H}_{22} \mathrm{NO}$ : 196.1701, found: 196.1694 .

\section{$\underline{N \text {-alkylation of bicyclic amine: }}$}

To a stirred solution of bicyclic amines $(0.17 \mathrm{~g}, 0.87 \mathrm{mmol})$ in dry acetonitrile $(10 \mathrm{~mL})$, anhydrous $\mathrm{K}_{2} \mathrm{CO}_{3}(0.36 \mathrm{~g}, 2.61 \mathrm{mmol})$ and $\mathrm{TMSCH}_{2} \mathrm{OTf}(0.18 \mathrm{~mL}, 0.914 \mathrm{mmol})$ were added at $0{ }^{\circ} \mathrm{C}$. The resulting solution was slowly warmed to room temperature and stirred for $1 \mathrm{~h}$ at room temperature. The resulting slurry was filtered and the residue was washed with EtOAc and concentrated the filtrate under reduced pressure. The crude compound was dissolved in hexane, filtered and dried under high vacuum which gave pure (by ${ }^{1} \mathrm{H}$ and $\left.{ }^{13} \mathrm{C}-\mathrm{NMR}\right) 7$ (mixture of two diastereomers) as a pale yellow color liquid, (small quantity was purified for taking NMR and other analytical data).

(4aR, 8aS)-4a-ethyl-8-((trimethylsilyl)methyl)-2-vinyloctahydro-2H-pyrano[2,3-b]pyridine (7b) :

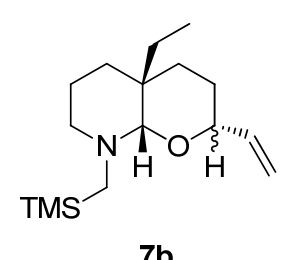

Yield: $0.24 \mathrm{~g}$, pale yellow color liquid.

TLC: $\mathrm{R}_{f}=\mathbf{0 . 5}\left(\mathrm{SiO}_{2}\right.$, ethyl acetate $:$ hexane $\left.=0.5: 9.5\right)$, as pale yellow color liquid.

IR (neat): 3434, 2940, 2861, 1645, 1445, 1298, $1247 \mathrm{~cm}^{-1}$.

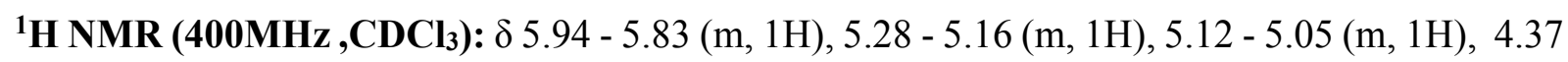
- $4.27(\mathrm{~m}, 0.5 \mathrm{H}), 3.70(\mathrm{~s}, 0.5 \mathrm{H}), 3.68$ - $3.64(\mathrm{~m}, 0.5 \mathrm{H}), 3.28(\mathrm{~m}, 0.5 \mathrm{H}), 2.89$ - $2.82(\mathrm{~m}, 0.5 \mathrm{H}), 2.76-$ $2.68(\mathrm{~m}, 0.5 \mathrm{H}), 2.56-2.42(\mathrm{~m}, 0.5 \mathrm{H}), 2.39-2.34(\mathrm{~m}, 0.5 \mathrm{H}), 2.33(\mathrm{~d}, J=14.6 \mathrm{~Hz}, 0.5 \mathrm{H}), 2.02$ 1.87 (m, $2.5 \mathrm{H}), 1.75$ - 1.69 (m, 2 H), 1.64 - 1.56 (m, 2 H), 1.49 - 1.36 (m, 3 H), 1.33 - 1.27 (m, 1 H), $1.11-1.05$ (m, $1 \mathrm{H}), 0.77$ (t, J=7.6 Hz, $3 \mathrm{H}), 0.04(\mathrm{~m}, 9 \mathrm{H})$. 
${ }^{13}$ C NMR (101MHz, CDCl 3$): \delta 139.7,139.6,114.9,113.8,95.9,76.5,69.7,49.2,46.1,43.3,35.1$, $34.9,33.9,29.3,28.7,27.4,26.1,25.7,23.1,21.4,21.1,7.0,6.9,-1.1,-1.5$.

HRMS (ESI): Calculated for $[\mathrm{M}+\mathrm{H}]^{+} \mathrm{C}_{16} \mathrm{H}_{32} \mathrm{NOSi}$ : 282.2253, found: 282.2246.

(4aS,8aR)-4a-ethyl-8-((trimethylsilyl)methyl)-2-vinyloctahydro-2H-pyrano[2,3-b]pyridine (7a)

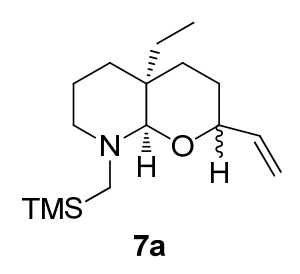

Yield: $0.19 \mathrm{~g}$, pale yellow color liquid.

${ }^{1} \mathbf{H}$ NMR (400MHz, $\left.\mathbf{C D C l}_{3}\right): \delta 6.02$ - $5.78(\mathrm{~m}, 1 \mathrm{H}), 5.28$ - $5.17(\mathrm{~m}, 1 \mathrm{H}), 5.12$ - $5.04(\mathrm{~m}, 1 \mathrm{H}), 4.37$ - $4.30(\mathrm{~m}, 0.5 \mathrm{H}), 3.76-3.63(\mathrm{~m}, 1 \mathrm{H}), 3.28$ (s., $0.5 \mathrm{H}), 2.89$ - $2.83(\mathrm{~m}, 0.5 \mathrm{H}), 2.77$ - $2.68(\mathrm{~m}, 0.5 \mathrm{H})$, $2.50(\mathrm{~d}, J=14.6 \mathrm{~Hz}, 0.5 \mathrm{H}), 2.41-2.34(\mathrm{~m}, 0.5 \mathrm{H}), 2.30(\mathrm{~d}, J=14.6 \mathrm{~Hz}, 0.5 \mathrm{H}), 2.08-1.88(\mathrm{~m}, 1.5$ H), 1.82 - $1.68(\mathrm{~m}, 2 \mathrm{H}), 1.67-1.52(\mathrm{~m}, 3 \mathrm{H}), 1.50-1.34(\mathrm{~m}, 3 \mathrm{H}), 1.30-1.20(\mathrm{~m}, 1 \mathrm{H}), 1.13$ - 1.04 $(\mathrm{m}, 1 \mathrm{H}), 0.77(\mathrm{t}, J=7.6 \mathrm{~Hz}, 3 \mathrm{H}), 0.05(\mathrm{~s}, 4.5 \mathrm{H}), 0.03(\mathrm{~s}, 4.5 \mathrm{H})$.

${ }^{13}$ C NMR (101MHz, CDC13): $\delta$ 139.7, 139.6, 114.9, 113.7, 95.9, 76.5, 69.7, 49.2, 46.1, 43.3, $35.1,34.9,33.9,29.3,28.7,27.4,26.1,25.7,23.1,21.4,21.1,6.9,6.8,-1.1,-1.5$.

HRMS (ESI): Calculated for $[\mathrm{M}+\mathrm{H}]^{+} \mathrm{C}_{16} \mathrm{H}_{32} \mathrm{NOSi}$ : 282.2253 found: 282.2249.

Synthesis of 1-((trimethylsilyl)methyl)piperidine-2-carbonitrile derivative (7):

TMSCN $(0.22 \mathrm{~mL}, 1.71 \mathrm{mmol})$ was added to a stirred solution of compound $7(0.24 \mathrm{~g}, 0.852$ mmol) in anhydrous dichloromethane at $0{ }^{\circ} \mathrm{C}$ and stirred for $15 \mathrm{~min}$. The reaction mixture was quenched with a saturated $\mathrm{NaHCO}_{3}$ solution, both the layers were separated and the organic layer was extracted with dichloromethane. The combined organic layer was concentrated, dried under high vacuum. The crude was dissolved in hexane and filtered. The filtrate was concentrated to obtain pure TMS-ether compound (0.32 g). 
This compound was dissolved in methanol $(20 \mathrm{~mL})$, PPTS (0.02 g, $0.082 \mathrm{mmol})$ was added to this solution and stirred at room temperature for $12 \mathrm{~h}$. On completion of starting material, the reaction mixture was concentrated and quenched with saturated $\mathrm{NaHCO}_{3}$ solution $(5 \mathrm{~mL})$, extracted with dichloromethane. The combined organic layer was dried over $\mathrm{Na}_{2} \mathrm{SO}_{4}$ and concentrated under reduced pressure. This crude compound was dissolved in hexane and filtered. The filtrate was concentrated and dried under vacuum to obtain pure compound 21 (0.24 g) (small quantity was purified for taking NMR and other analytical data).

\section{(3R)-3-ethyl-3-(3-hydroxypent-4-en-1-yl)-1-((trimethylsilyl)methyl)piperidine-2-}

carbonitrile (21 b):

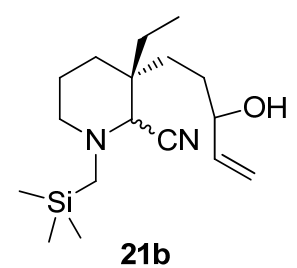

Yield: $0.24 \mathrm{~g}$; pale yellow color liquid.

TLC: $\mathrm{R}_{f}=\mathbf{0 . 5}\left(\mathrm{SiO}_{2}\right.$, ethyl acetate $:$ hexane $\left.=1: 9\right)$.

IR (neat): 3435, 3019, 2944, 2863, 2399, 1422, $1216 \mathrm{~cm}^{-1}$.

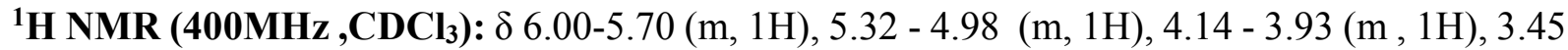
- $3.28(\mathrm{~m}, 1 \mathrm{H}), 2.76$ - $2.54(\mathrm{~m}, 1 \mathrm{H}), 2.33$ - $2.16(\mathrm{~m}, 1 \mathrm{H}), 2.09$ - $1.99(\mathrm{~m}, 1 \mathrm{H}), 1.94-1.74(\mathrm{~m}, 3 \mathrm{H})$, $1.68-1.59(\mathrm{~m}, 1 \mathrm{H}), 1.52$ - $1.36(\mathrm{~m}, 6 \mathrm{H}), 1.32$ - $1.14(\mathrm{~m}, 2 \mathrm{H}), 0.85$ - $0.73(\mathrm{~m}, 3 \mathrm{H}), 0.06(\mathrm{~s}, 9 \mathrm{H})$;

${ }^{13} \mathbf{C}$ NMR (101MHz ,CDCl $\left.\mathbf{3}\right): \delta 140.9,140.7,140.6,115.5,115.3,115.1,73.7,73.6,73.4,65.8$, $65.7,65.6,52.0,51.9,51.8,48.5,38.3,38.2,32.2,32.0,30.2,30.1,29.5,29.3,29.3,25.5,23.0$, $20.9,7.2,6.6,-1.6,-1.5$

HRMS (ESI): Calculated for $[\mathrm{M}+\mathrm{H}]^{+} \mathrm{C}_{17} \mathrm{H}_{33} \mathrm{~N}_{2} \mathrm{OSi}$ : 309.2362 found: 309.2357 . 
(3S)-3-ethyl-3-(3-hydroxypent-4-en-1-yl)-1-((trimethylsilyl)methyl)piperidine-2-carbonitrile (21a):

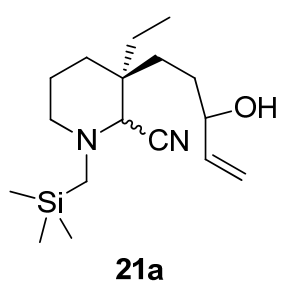

Yield: $0.19 \mathrm{~g}$; pale yellow color liquid.

${ }^{1} \mathbf{H}$ NMR (400MHz, $\left.\mathbf{C D C l}_{3}\right): \delta 5.97-5.69(\mathrm{~m}, 1 \mathrm{H}), 5.30-5.05(\mathrm{~m}, 1 \mathrm{H}), 4.12-3.97(\mathrm{~m}, 1 \mathrm{H})$, 3.44 - $3.29(\mathrm{~m}, 1 \mathrm{H}), 2.72$ - $2.56(\mathrm{~m}, 1 \mathrm{H}), 2.32$ - $2.16(\mathrm{~m}, 1 \mathrm{H}), 2.11$ - $1.97(\mathrm{~m}, 1 \mathrm{H}), 1.94$ - 1.77 (m, $3 \mathrm{H}), 1.71-1.62(\mathrm{~m}, 1 \mathrm{H}), 1.51-1.38(\mathrm{~m}, 6 \mathrm{H}), 1.28-1.14(\mathrm{~m}, 2 \mathrm{H}), 0.86-0.74(\mathrm{~m}, 3 \mathrm{H}), 0.06(\mathrm{~s}$, 9H).

${ }^{13} \mathbf{C}$ NMR (101MHz ,CDCl $\left.\mathbf{3}\right): \delta 140.9,140.6,115.5,115.3,115.2,73.7,73.6,73.4,65.7,65.6$, $65.6,51.9,51.8,48.5,38.3,38.2,32.2,32.0,30.2,30.1,29.5,29.3,29.3,25.4,23.0,20.9,7.2$, $6.6,-1.6,-1.5$.

HRMS (ESI): Calculated for $[\mathrm{M}+\mathrm{H}]^{+} \quad \mathrm{C}_{17} \mathrm{H}_{33} \mathrm{~N}_{2} \mathrm{OSi}$ : 309.2362 found: 309.2356 .

\section{Oxidation of Allylic alcohol to enone:}

To a solution of 21 ( $0.24 \mathrm{~g}, 0.78 \mathrm{mmol})$ in dry DCM (5 mL), Dess-Martin Periodinane ( $0.4 \mathrm{~g}, 0.94$ mmol) was added at $0{ }^{\circ} \mathrm{C}$. The reaction was allowed to warm to room temperature and stirred for $30 \mathrm{~min}$. The reaction was quenched successively with saturated aqueous $\mathrm{Na}_{2} \mathrm{~S}_{2} \mathrm{O}_{3}(5 \mathrm{~mL})$ and saturated aqueous $\mathrm{NaHCO}_{3}(5 \mathrm{~mL})$ solution, stirred for $15 \mathrm{~min}$. The mixture was extracted with dichloromethane $(3 \times 15 \mathrm{~mL})$ and the combined organic layer was dried over $\mathrm{Na}_{2} \mathrm{SO}_{4}$ and concentrated. This crude compound was dissolved in hexane and filtered. The filtrate was concentrated and dried under vacuum to obtain pure compound 22 (small quantity was purified for taking NMR and other analytical data). 
(3R)-3-ethyl-3-(3-oxopent-4-en-1-yl)-1-((trimethylsilyl)methyl)piperidine-2-carbonitrile (22b) :

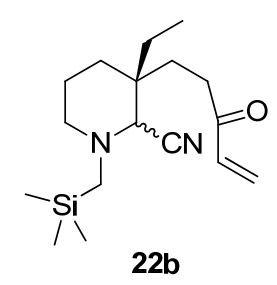

Yield: $0.22 \mathrm{~g}$; colorless liquid.

TLC: $\mathrm{R}_{f}=\mathbf{0 . 4}\left(\mathrm{SiO}_{2}\right.$, ethylacetate $:$ hexane= $\left.1: 9\right)$.

IR (neat): 3019, 2949, 2794, 1682, 1617, 1464, 1402, 1249, $1157 \mathrm{~cm}^{-1}$.

${ }^{1} \mathbf{H}$ NMR (400MHz, $\left.\mathbf{C D C l}_{3}\right): \delta 6.43-6.30(\mathrm{~m}, 1 \mathrm{H}), 6.28-6.19(\mathrm{~m}, 1 \mathrm{H}), 5.88-5.81(\mathrm{~m}, 1 \mathrm{H}), 3.41$ $(\mathrm{s}, 0.5 \mathrm{H}), 3.38(\mathrm{~s}, 0.5 \mathrm{H}), 2.72-2.61(\mathrm{~m}, 1 \mathrm{H}), 2.58-2.38(\mathrm{~m}, 2 \mathrm{H}), 2.29-2.19(\mathrm{~m},, 1 \mathrm{H}), 2.05(\mathrm{~d}, J$ $=14.6 \mathrm{~Hz}, 1 \mathrm{H}), 1.90(\mathrm{~d}, J=14.6 \mathrm{~Hz}, 1 \mathrm{H}), 1.85-1.63(\mathrm{~m}, 3 \mathrm{H}), 1.56-1.39(\mathrm{~m}, 4 \mathrm{H}), 1.29-1.18(\mathrm{~m}$, $1 \mathrm{H}), 0.88-0.74(\mathrm{~m}, 3 \mathrm{H}), 0.07(\mathrm{~s}, 4.5 \mathrm{H}), 0.06(\mathrm{~s}, 4.5 \mathrm{H})$.

${ }^{13}$ C NMR (101MHz, $\left.\mathbf{C D C l}_{3}\right): \delta$ 200.0, 199.8, 136.2, 136.1, 128.4, 128.1, 115.5, 115.3, 65.7, 65.6, 51.8, 51.7, 48.6, 48.5, 38.3, 38.2, 33.6, 32.9, 30.5, 29.6, 29.5, 29.1, 24.1, 23.1, 20.9, 20.8, 7.2, 6.6, $-1.6,-1.5$.

HRMS (ESI): Calculated for [M + H] $]^{+} \mathrm{C}_{17} \mathrm{H}_{31} \mathrm{~N}_{2} \mathrm{OSi}$ : 307.2206 found:307.2199.

(3S)-3-ethyl-3-(3-oxopent-4-en-1-yl)-1-((trimethylsilyl)methyl)piperidine-2-carbonitrile (22a) :

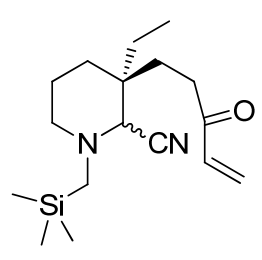

$22 \mathrm{a}$ 
Yield: $0.17 \mathrm{~g}$; colorless liquid.

${ }^{1} \mathbf{H}$ NMR (400MHz, $\left.\mathbf{C D C l}_{3}\right): \delta$ 6.47-6.35 (m, 1H), 6.26-6.15 (m, 1H), 5.89-5.78 (m, 1H), 3.41 (s, $0.5 \mathrm{H}), 3.38(\mathrm{~s}, 0.5 \mathrm{H}), 2.75-2.60(\mathrm{~m}, 1 \mathrm{H}), 2.56-2.37(\mathrm{~m}, 2 \mathrm{H}), 2.30-2.20(\mathrm{~m}, 1 \mathrm{H}), 2.05(\mathrm{~d}, J=$ $14.4 \mathrm{~Hz}, 1 \mathrm{H}), 1.90(\mathrm{~d}, J=14.4 \mathrm{~Hz}, 1 \mathrm{H}), 1.83-1.60(\mathrm{~m}, 3 \mathrm{H}), 1.56-1.38$ (m, 4H), $1.29-1.19$ (m, $1 \mathrm{H}), 0.85-0.74(\mathrm{~m}, 3 \mathrm{H}), 0.06(\mathrm{~s}, 9 \mathrm{H})$.

${ }^{13} \mathbf{C}$ NMR (101MHz, $\left.\mathbf{C D C l}_{3}\right): \delta 200.0,199.8,136.2,136.1,128.4,128.1,115.5,115.3,65.6,65.5$, $51.8,51.7,48.6,48.5,38.3,38.2,33.6,32.9,30.5,29.5,29.1,24.0,23.1,20.9,20.8,7.2,6.6,-1.6$, -1.5 .

HRMS (ESI): Calculated for [M + H] ${ }^{+} \mathrm{C}_{17} \mathrm{H}_{31} \mathrm{~N}_{2} \mathrm{OSi}$ : 307.2206 found: 307.2200 .

\section{$\underline{\text { Intramolecuclar }[3+2] \text { cycloaddition reaction: }}$}

To a stirred solution of $22(0.12 \mathrm{~g}, 0.391 \mathrm{mmol})$ in anhydrous acetonitrile, $\mathrm{Ag}(\mathrm{I}) \mathrm{F}(0.06 \mathrm{~g}, 0.47$ mmol) was added at room temperature and stirred for $3 \mathrm{~h}$ ( protect reaction mixture from light). On completion of reaction (TLC analysis), the reaction mixture was filtered through celite and washed with EtOAc. The combined organic layer was concentrated and purification by column chromatography resulted $\mathbf{5}$ as colorless liquid.

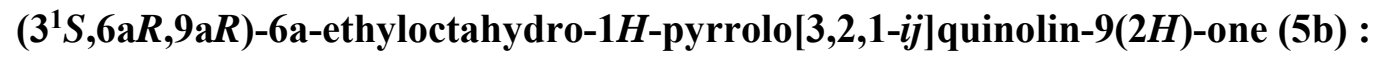

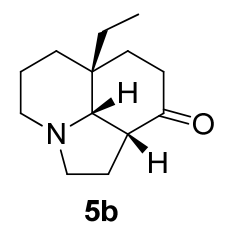

Colorless liquid; Yield: $0.075 \mathrm{~g}, 92 \%$.

TLC: $\mathrm{R}_{\mathrm{f}}=\mathbf{0 . 4}\left(\mathrm{SiO}_{2}\right.$, ethylacetate $:$ hexane $\left.=6: 4\right)$.

$[\boldsymbol{\alpha}]^{24} \mathbf{D}=-23.81 \quad\left(c=0.5, \mathrm{CHCl}_{3}\right)$.

IR (neat): $3438,2934,2786,2725,1709,1448,1345 \mathrm{~cm}^{-1}$;

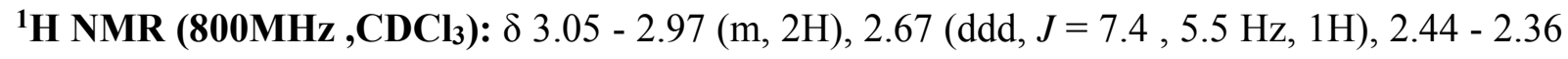
(m, 2H), 2.33 - $2.29(\mathrm{~m}, 1 \mathrm{H}), 2.25(\mathrm{td}, J=13.7,4.3 \mathrm{~Hz}, 1 \mathrm{H}), 1.96-1.92(\mathrm{~m}, 1 \mathrm{H}), 1.92-1.87$ (m, 2H), 1.82 - $1.79(\mathrm{~m}, 1 \mathrm{H}), 1.74-1.67(\mathrm{~m}, 1 \mathrm{H}), 1.67$ - $1.60(\mathrm{~m}, 2 \mathrm{H}), 1.52$ - 1.47 (m, 2H), 1.32 (app sextet, $J=7.4,1 \mathrm{H}), 1.10(\mathrm{td}, J=13.5,4.7 \mathrm{~Hz}, 1 \mathrm{H}), 0.94(\mathrm{t}, J=7.6 \mathrm{~Hz}, 3 \mathrm{H})$. 
${ }^{13}$ C NMR (101MHz, $\left.\mathbf{C D C l}_{3}\right): \delta 211.5,73.5,53.2,52.9,48.1,36.8,34.7,32.8,30.1,26.0,21.3$, 21.2, 7.1.

HRMS (ESI): Calculated for $[\mathrm{M}+\mathrm{H}]^{+} \mathrm{C}_{13} \mathrm{H}_{22} \mathrm{NO}$ : 208.1701, found: 208.1693.

$\left(3^{1} R, 6 a S, 9 a S\right)-6 a-e t h y l o c t a h y d r o-1 H$-pyrrolo[3,2,1-ij]quinolin-9(2H)-one (5a) :

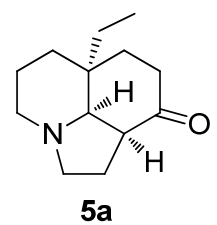

colorless liquid; Yield: $0.045 \mathrm{~g}, 91 \%$;

TLC: $\mathrm{R}_{\mathrm{f}}=\mathbf{0 . 4}\left(\mathrm{SiO}_{2}\right.$, ethyl acetate: hexane $\left.=6: 4\right)$;

$[\boldsymbol{\alpha}]^{24} \mathrm{D}=+21.62\left(c=0.5, \mathrm{CHCl}_{3}\right)$

${ }^{1}$ H NMR (400MHz, CDCl $)$ ): $\delta 3.07$ - 2.96 (m, 2H), 2.67 (ddd, $J=7.3$, $\left.5.3 \mathrm{~Hz}, 1 \mathrm{H}\right), 2.47$ - 2.19 (m, 4H), 1.98 - $1.62(\mathrm{~m}, 7 \mathrm{H}), 1.54-1.44(\mathrm{~m}, 2 \mathrm{H}), 1.37$ - 1.28 (app sextet, $7.4 \mathrm{~Hz}, 1 \mathrm{H}), 1.10$ (td, $J$ $=13.3,4.5 \mathrm{~Hz}, 1 \mathrm{H}), 0.94(\mathrm{t}, J=7.6 \mathrm{~Hz}, 3 \mathrm{H})$.

${ }^{13}$ C NMR (101MHz, $\left.\mathbf{C D C l}_{3}\right): \delta 211.5,73.6,53.2,52.9,48.2,36.8,34.7,32.9,30.1,26.1,21.3$, $21.2,7.1$.

HRMS (ESI): Calculated for $[\mathrm{M}+\mathrm{H}]^{+} \mathrm{C}_{13} \mathrm{H}_{22} \mathrm{NO}$ : 208.1701, found: 208.1694.

Synthesis of (+)-Aspidospermidine (4a):

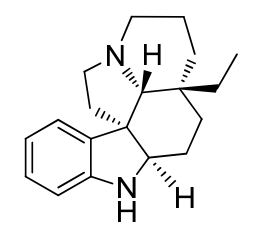

(+) Aspidospermidine (4a)

Phenyl hydrazine $(0.022 \mathrm{~g}, 0.202 \mathrm{mmol})$ was added to a stirred solution of $\mathbf{5 b}(0.035 \mathrm{~g}, 0.168$ mmol) in benzene $(5 \mathrm{~mL})$ and refluxed for $3 \mathrm{~h}$. The reaction mixture was cooled to room temperature and concentrated to obtain crude phenyl hydrazone, which was dissolved in $5 \mathrm{~mL}$ glacial acetic acid and refluxed for $4 \mathrm{~h}$, concentrated to dryness to obtain crude indolene (dehydroaspidospermidine) as a brown color liquid. 
This crude indolene was dissolved in anhydrous THF $(5 \mathrm{~mL})$, added $\mathrm{LiAlH}_{4}(0.059 \mathrm{~g}, 1.68 \mathrm{mmol})$, and refluxed for $12 \mathrm{~h}$. The reaction mixture was cooled to $0{ }^{\circ} \mathrm{C}$, and quenched with $\mathrm{H}_{2} \mathrm{O}(2 \mathrm{~mL})$, stirred for $15 \mathrm{~min}$. The suspention was filtered through a plug of celite and the filtrate was dried over $\mathrm{Na}_{2} \mathrm{SO}_{4}$ and concentrated. The resulting residue was purified by column chromatography using ethylacetate to give aspidospermidine $4 \mathbf{a}(0.024 \mathrm{~g}, 50 \%)$ as a pale yellow oil. This compound 4a on standing long time gave pale yellow color solid.

Optical Rotation: $[\alpha]_{\mathbf{D}}{ }^{24}=+20.14(c=0.5, \mathrm{EtOH}) ; \mathrm{Lit}^{[8 a]}[\boldsymbol{\alpha}]_{\mathbf{D}}{ }^{29}=+20.6(c=0.64, \mathrm{EtOH})$.

Melting Point: $118-120^{\circ} \mathrm{C}$.

TLC: $\mathrm{R}_{f}=\mathbf{0 . 4}\left(\mathrm{SiO}_{2}, \mathrm{DCM}: \mathrm{MeOH}=9: 1\right)$.

IR (film): 3363, 2932, 2860, 2779, 2722, 1606, 1480, 1462, 1332.

${ }^{1} \mathbf{H}$ NMR (800MHz ,CDCl 3$): \delta 7.09(\mathrm{~d}, J=7.5 \mathrm{~Hz}, 1 \mathrm{H}), 7.02(\mathrm{td}, J=7.5,0.95 \mathrm{~Hz}, 1 \mathrm{H}), 6.74(\mathrm{t}$, $J=7.3 \mathrm{~Hz}, 1 \mathrm{H}), 6.65(\mathrm{~d}, J=7.5 \mathrm{~Hz}, 1 \mathrm{H}), 3.52(\mathrm{dd}, J=11.3,6.3 \mathrm{~Hz}, 1 \mathrm{H}), 3.15-3.11(\mathrm{~m}, 1 \mathrm{H})$, 3.08 - $3.04(\mathrm{~m}, 1 \mathrm{H}), 2.33-2.28(\mathrm{~m}, 1 \mathrm{H}), 2.28$ - $2.24(\mathrm{~m}, 1 \mathrm{H}), 2.23(\mathrm{~s}, 1 \mathrm{H}), 1.99-1.92(\mathrm{~m}, 2 \mathrm{H})$, $1.74(\mathrm{qt}, J=13.04,4.06 \mathrm{~Hz}, 1 \mathrm{H}), 1.67-1.62(\mathrm{~m}, 2 \mathrm{H}), 1.54-1.46(\mathrm{~m}, 3 \mathrm{H}), 1.43-1.36(\mathrm{~m}, 1 \mathrm{H})$, $1.12(\mathrm{td}, J=13.7,4.8 \mathrm{~Hz}, 1 \mathrm{H}), 1.06(\mathrm{dt}, J=13.6,3.8 \mathrm{~Hz}, 1 \mathrm{H}), 0.90-0.85(\mathrm{~m}, 1 \mathrm{H}), 0.64(\mathrm{t}, J=$ $7.5 \mathrm{~Hz}, 3 \mathrm{H})$.

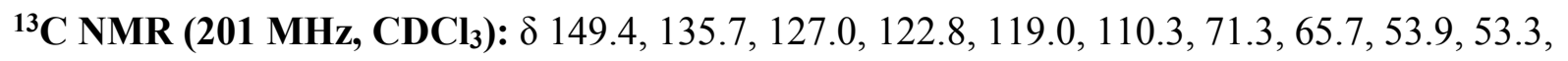
$53.0,38.8,35.6,34.4,30.0,28.1,23.0,21.7,6.8$.

HRMS (ESI): Calculated for $[\mathrm{M}+\mathrm{H}]^{+} \mathrm{C}_{19} \mathrm{H}_{27} \mathrm{~N}_{2}$ : 283.2174, found: 283.2167 . 


\section{III. ${ }^{1} \mathrm{H}$ and ${ }^{13} \mathrm{C}$ NMR spectra of compounds and other analytical data.}




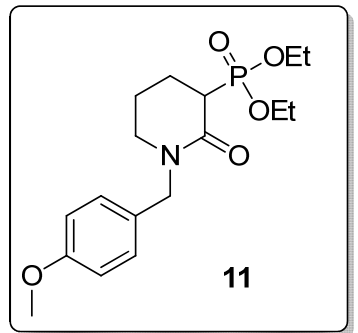

${ }^{1} \mathrm{H}-\mathrm{NMR}\left(800 \mathrm{MHz}, \mathrm{CDCl}_{3}\right)$
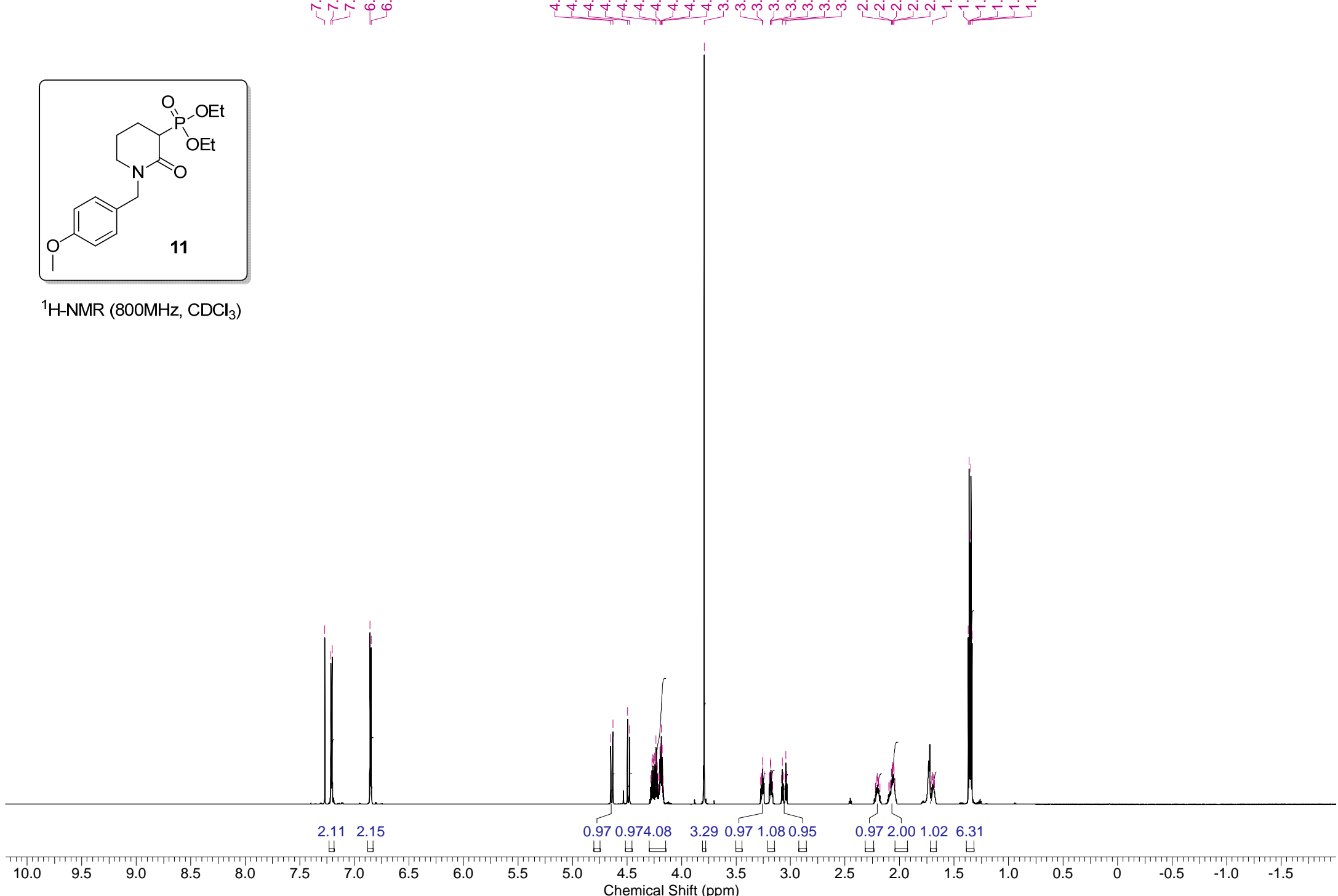


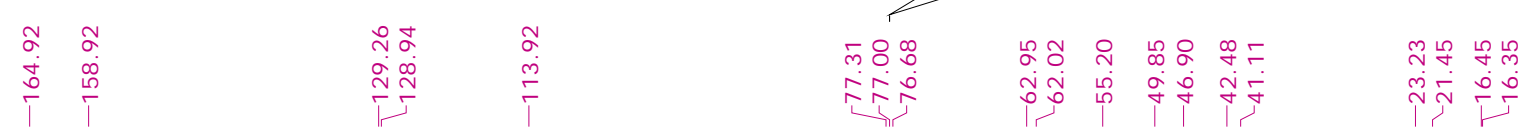

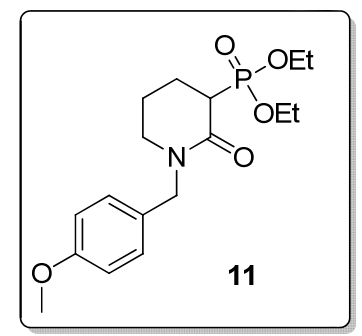

${ }^{3} \mathrm{C}-\mathrm{NMR}(101 \mathrm{MHz}, \mathrm{CDCl})$ 


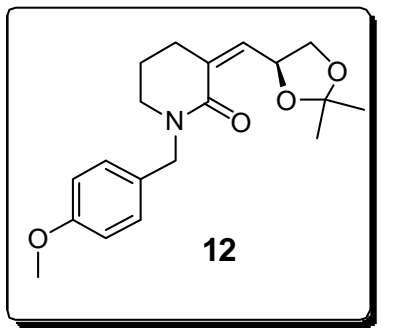

1H-NMR (800MHz, CDCl3)

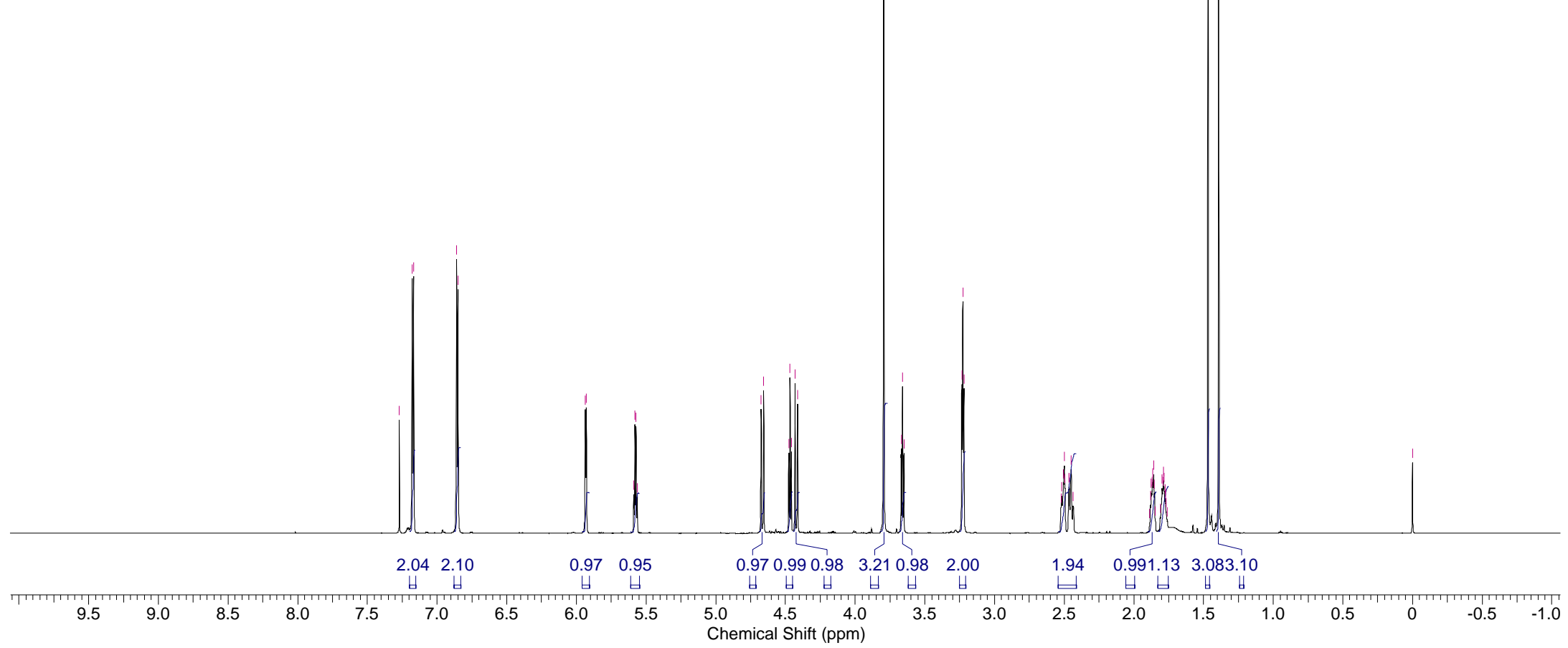


pmbw1 13c_123A.005.001.1r.esp
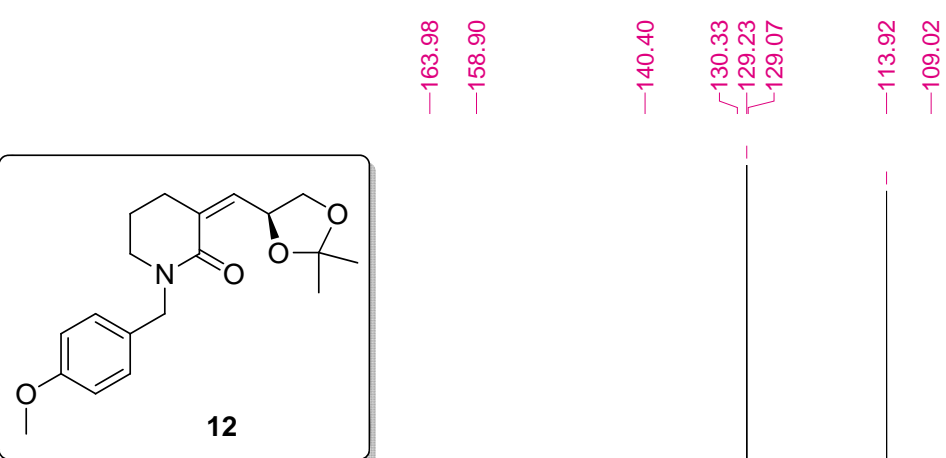

${ }^{13} \mathrm{C}-\mathrm{NMR}\left(101 \mathrm{MHz}, \mathrm{CDCl}_{3}\right)$
CHLOROFORM-d
$\stackrel{\infty}{\rightarrow}$

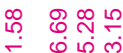

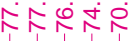
官 字守
ले ํํำ

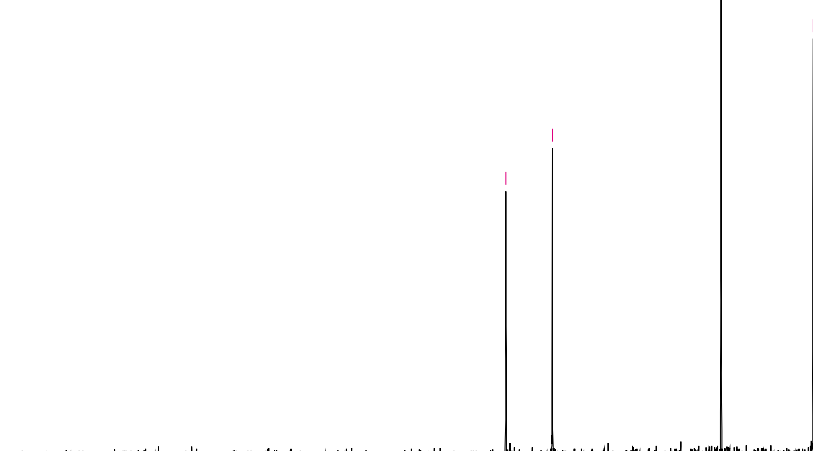

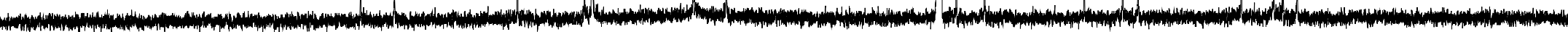
$210 \quad 200 \quad 190$
$180 \quad 170$
150
$40 \quad 130 \quad 120$
$\begin{array}{lll}110 & 100 \quad 90\end{array}$
$80 \quad 70 \quad 60$
$50 \quad 40$
$30 \quad 20$ 


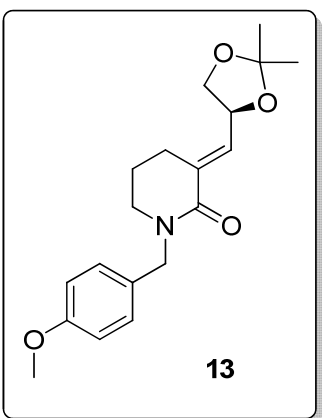

${ }^{1} \mathrm{H}-\mathrm{NMR}\left(800 \mathrm{MHz}, \mathrm{CDCl}_{3}\right)$

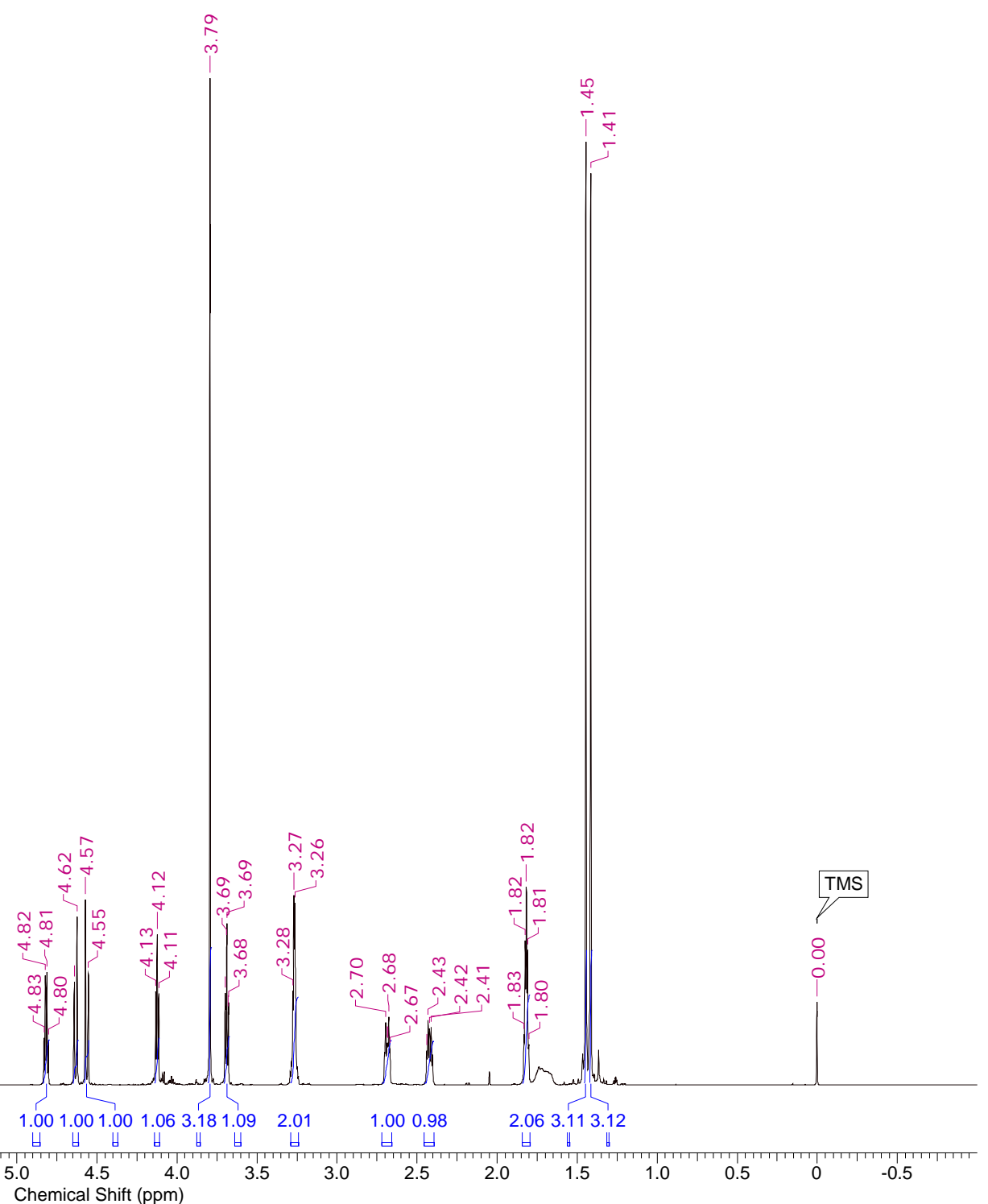


skb-w2-processed.002.001.1r.esp

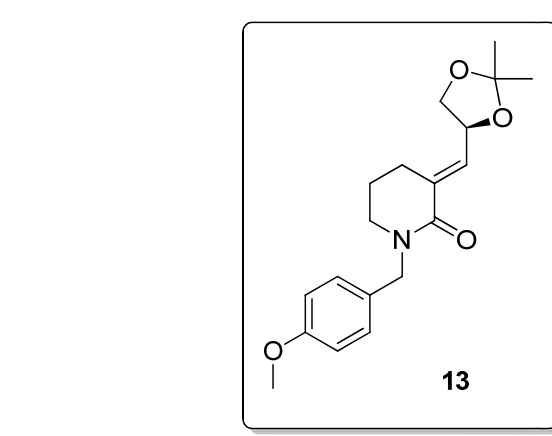

${ }^{13} \mathrm{C}-\mathrm{NMR}\left(101 \mathrm{MHz}, \mathrm{CDCl}_{3}\right)$

CHLOROFORM-d

ขึะ:

(1)

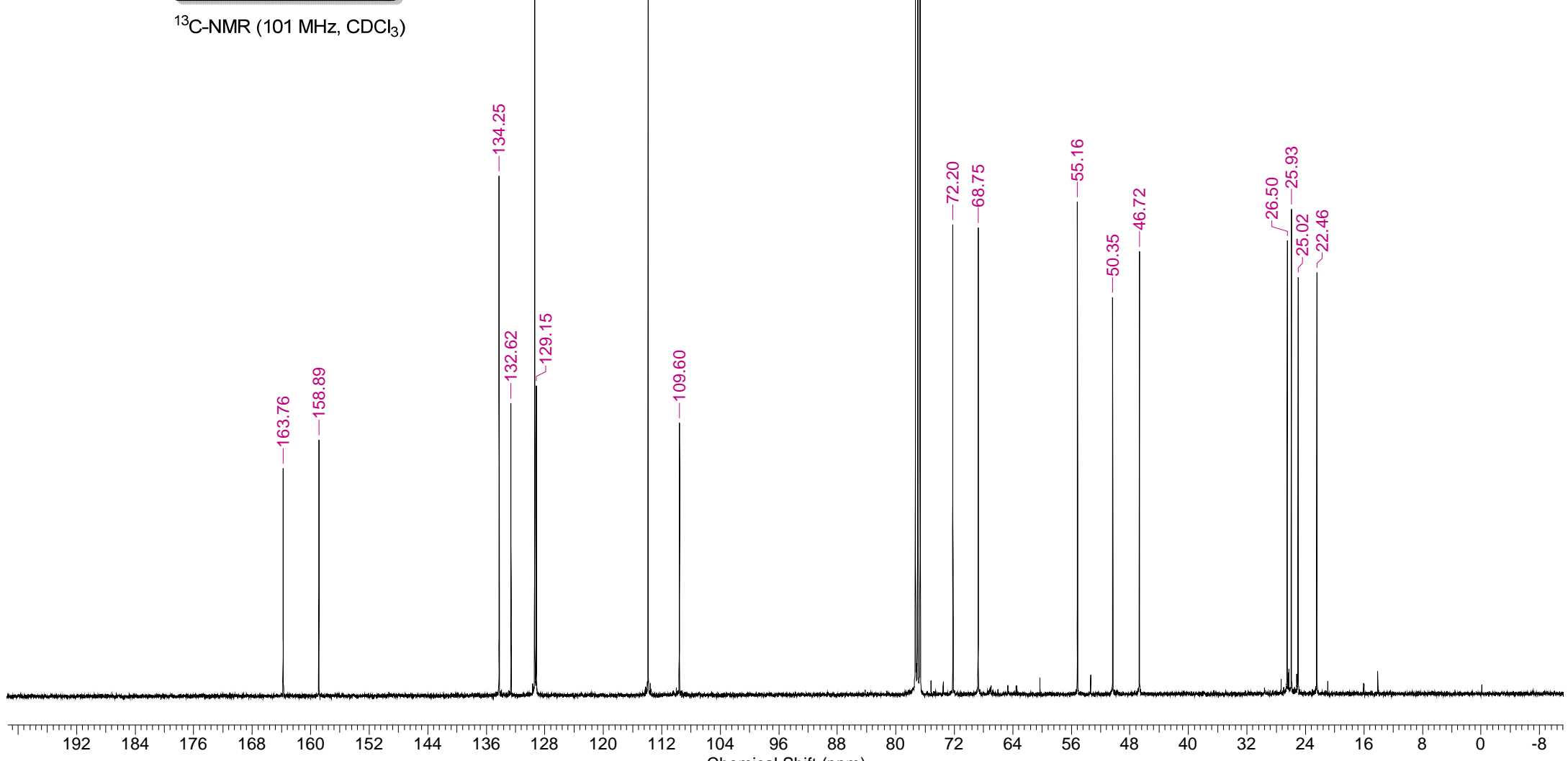

$\begin{array}{llllllllllllll}192 & 184 & 176 & 168 & 160 & 152 & 144 & 136 & 128 & 120 & 112 & 104 & 96 & 88 \\ & & & & & & & & \end{array}$ 

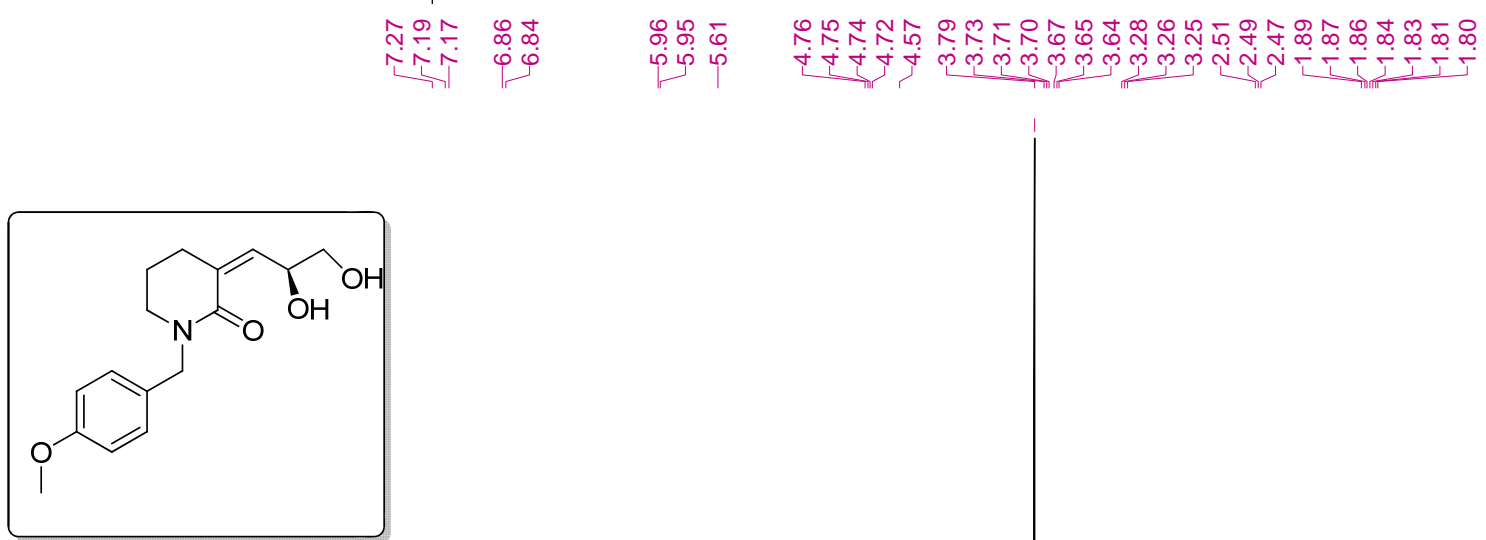

${ }^{1} \mathrm{H}-\mathrm{NMR}\left(400 \mathrm{MHz}, \mathrm{CDCl}_{3}\right.$ )

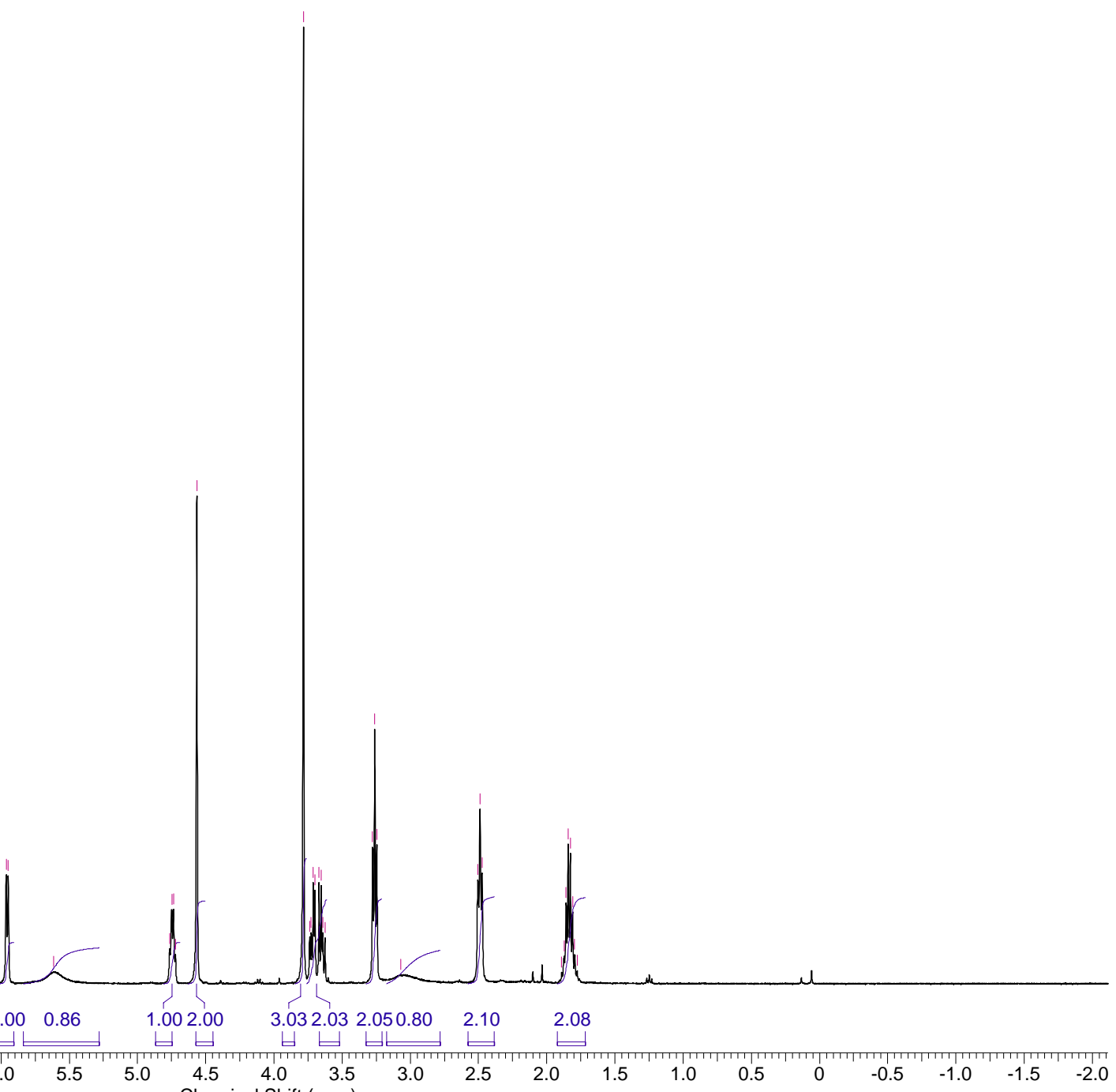


skb 02 155_D1.004.001.1r.esp

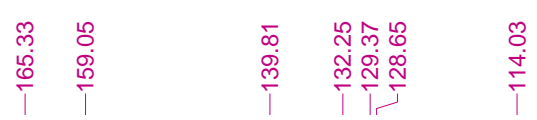

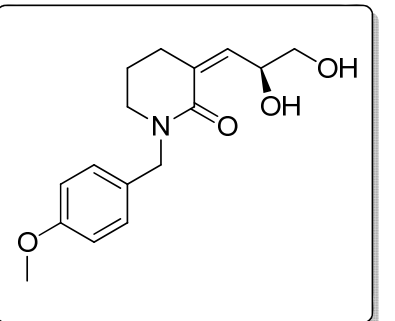

${ }^{13} \mathrm{C}-\mathrm{NMR}\left(101 \mathrm{MHz}, \mathrm{CDCl}_{3}\right)$
CHLOROFORM-d

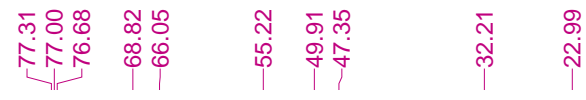

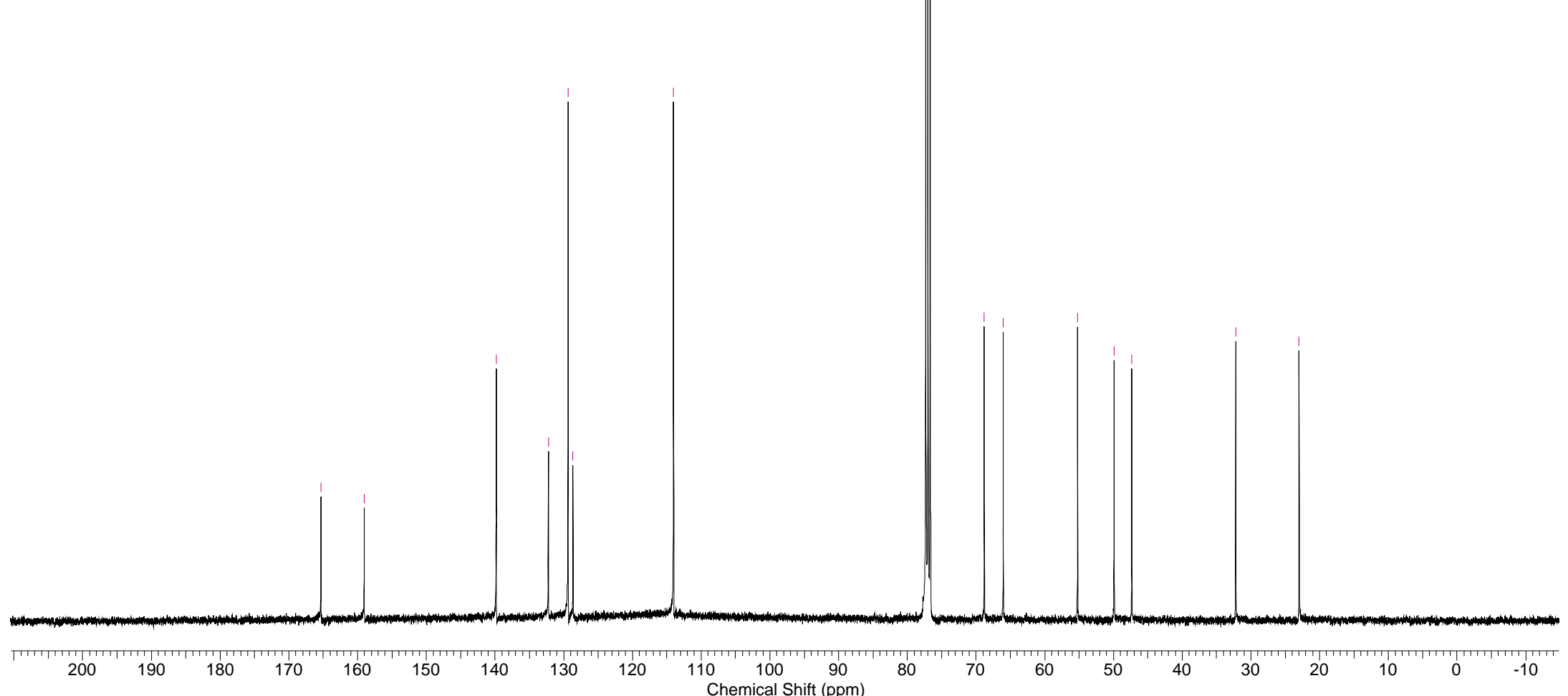




\section{NOESY Spectra of compound 10:}

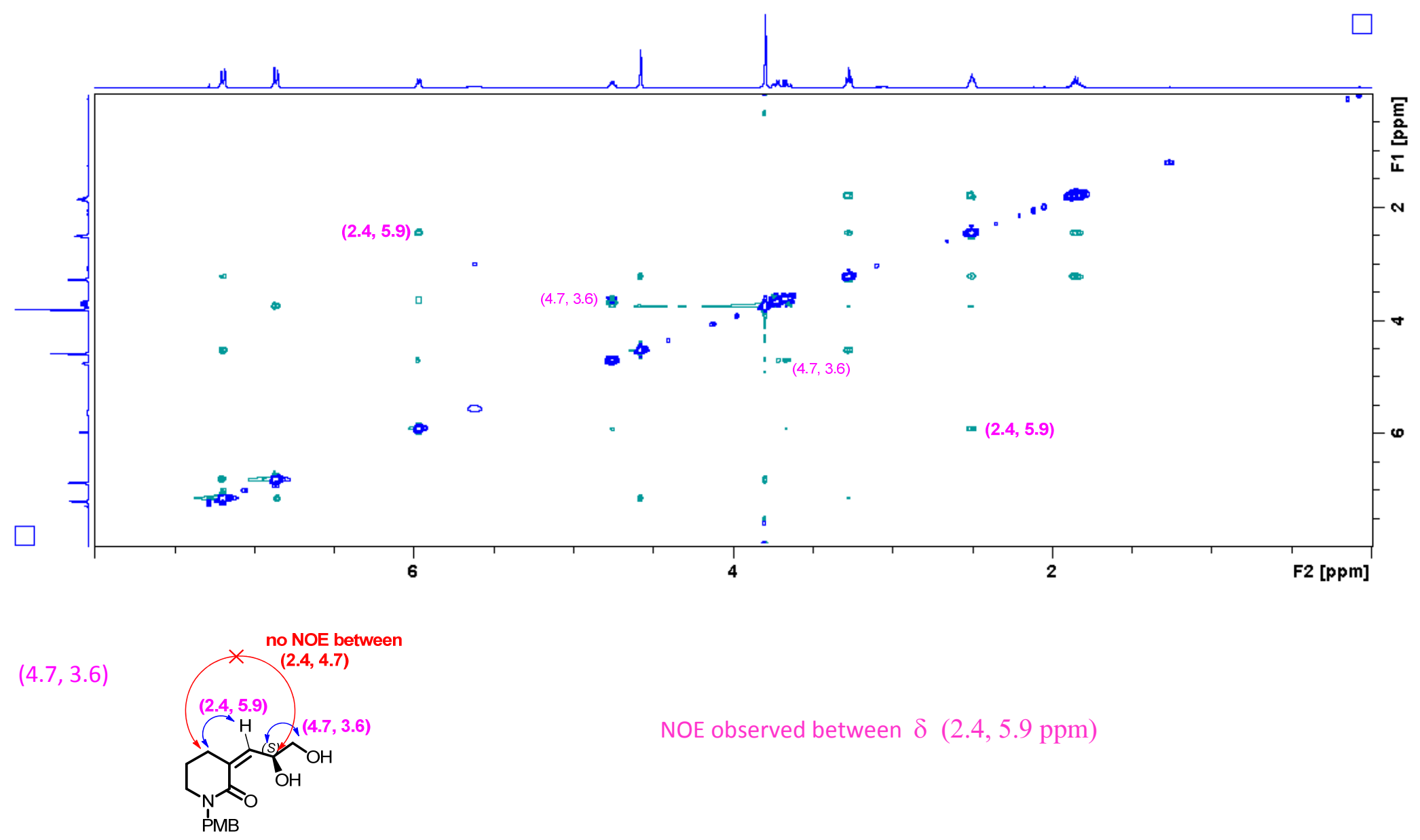

From Noesy spectrum, it is clear that the olefinic proton ( $\delta 5.9 \mathrm{ppm})$ is in close proximate with allylic protons $(2.4 \mathrm{ppm})$ 


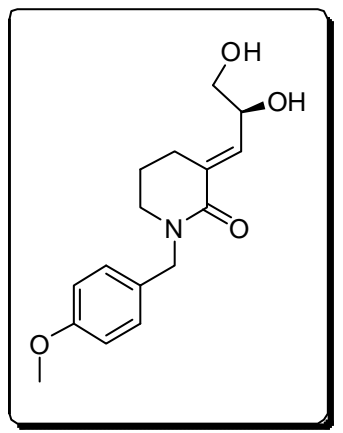

${ }^{1} \mathrm{H}-\mathrm{NMR}\left(400 \mathrm{MHz}, \mathrm{CDCl}_{3}\right)$

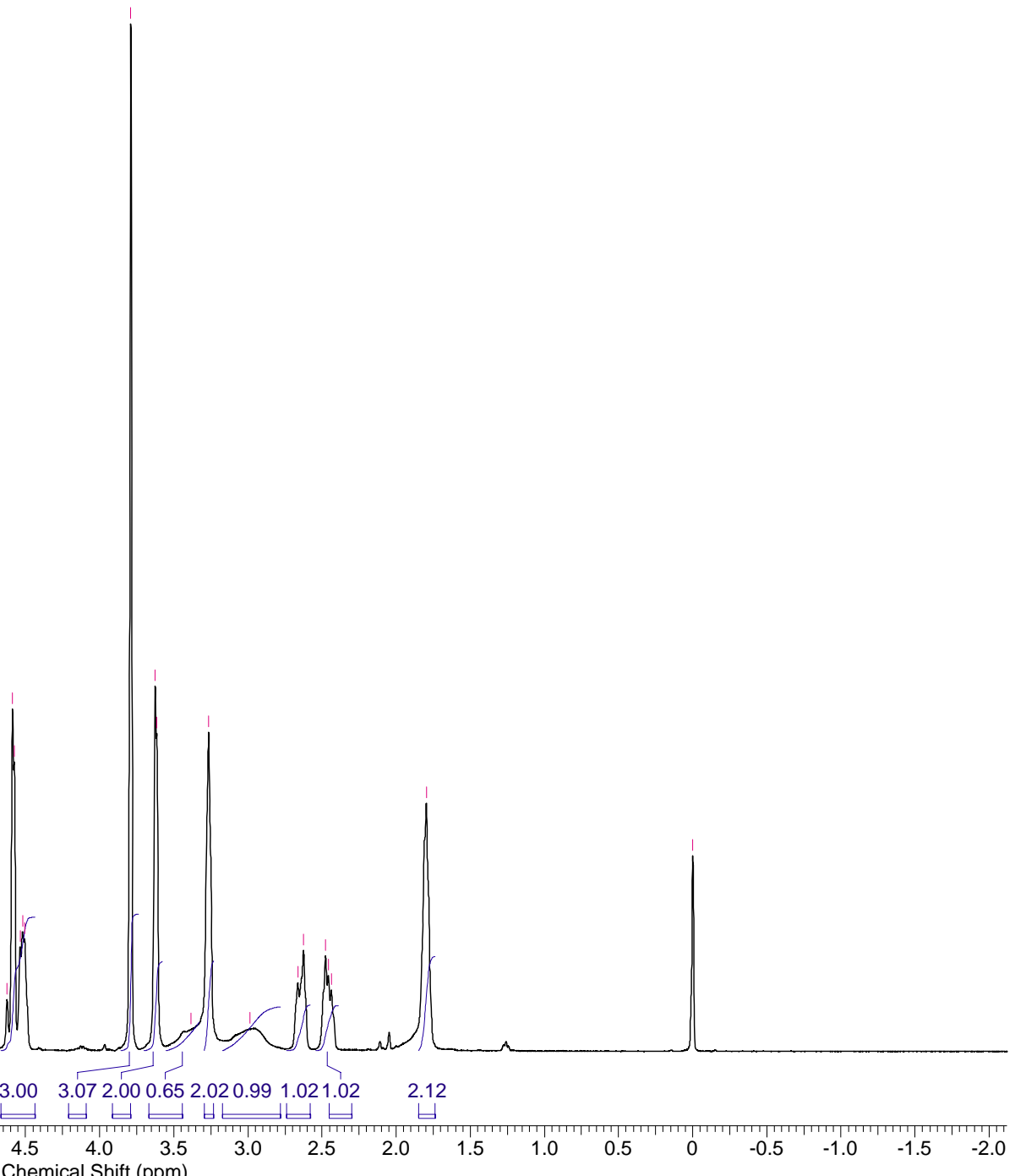


skb 02 156-D2.005.001.1r.esp

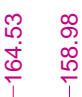
กิ เุ ษ

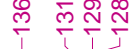
$\stackrel{\hat{m}}{\mathrm{G}}$

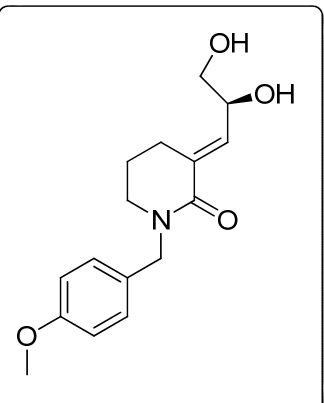

${ }^{13} \mathrm{C}-\mathrm{NMR}\left(101 \mathrm{MHz}, \mathrm{CDCl}_{3}\right)$
CHLOROFORM-d

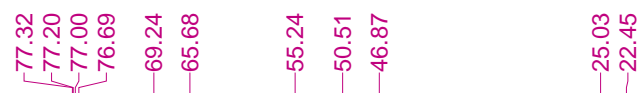

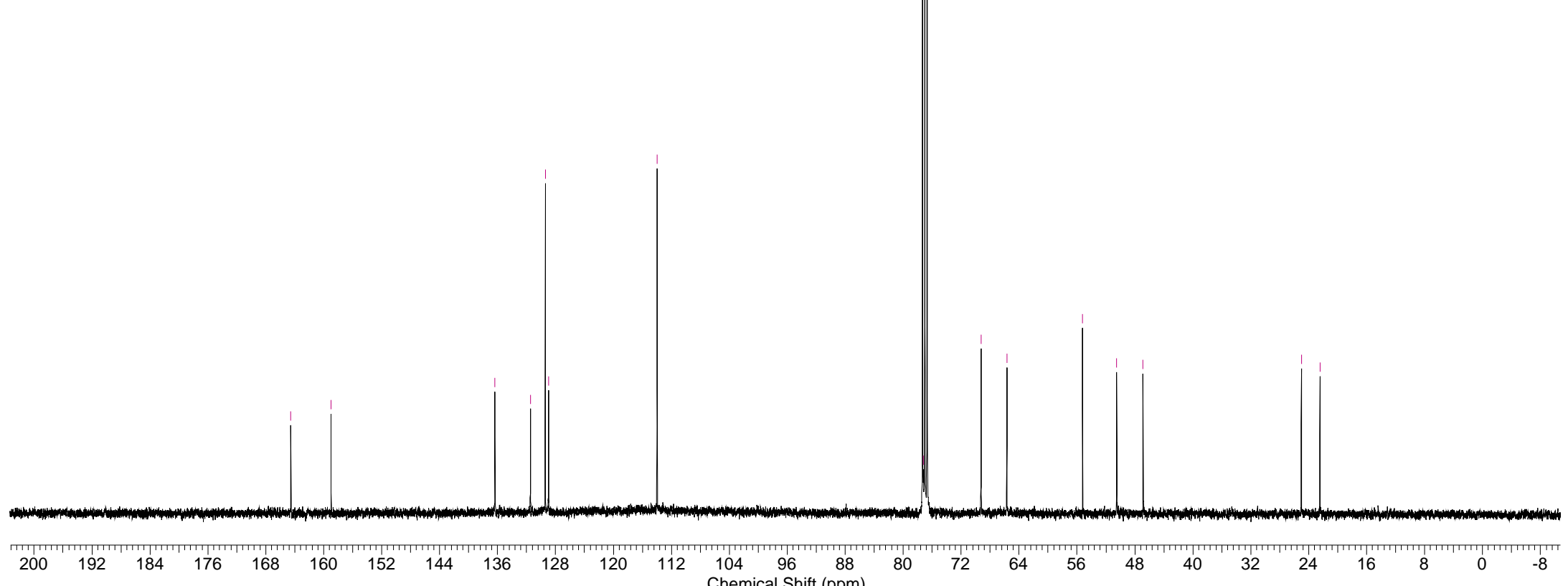




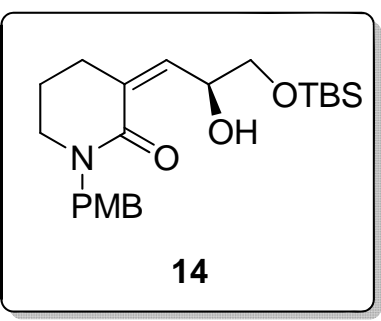

${ }^{1} \mathrm{H}-\mathrm{NMR}\left(400 \mathrm{MHz}, \mathrm{CDCl}_{3}\right)$

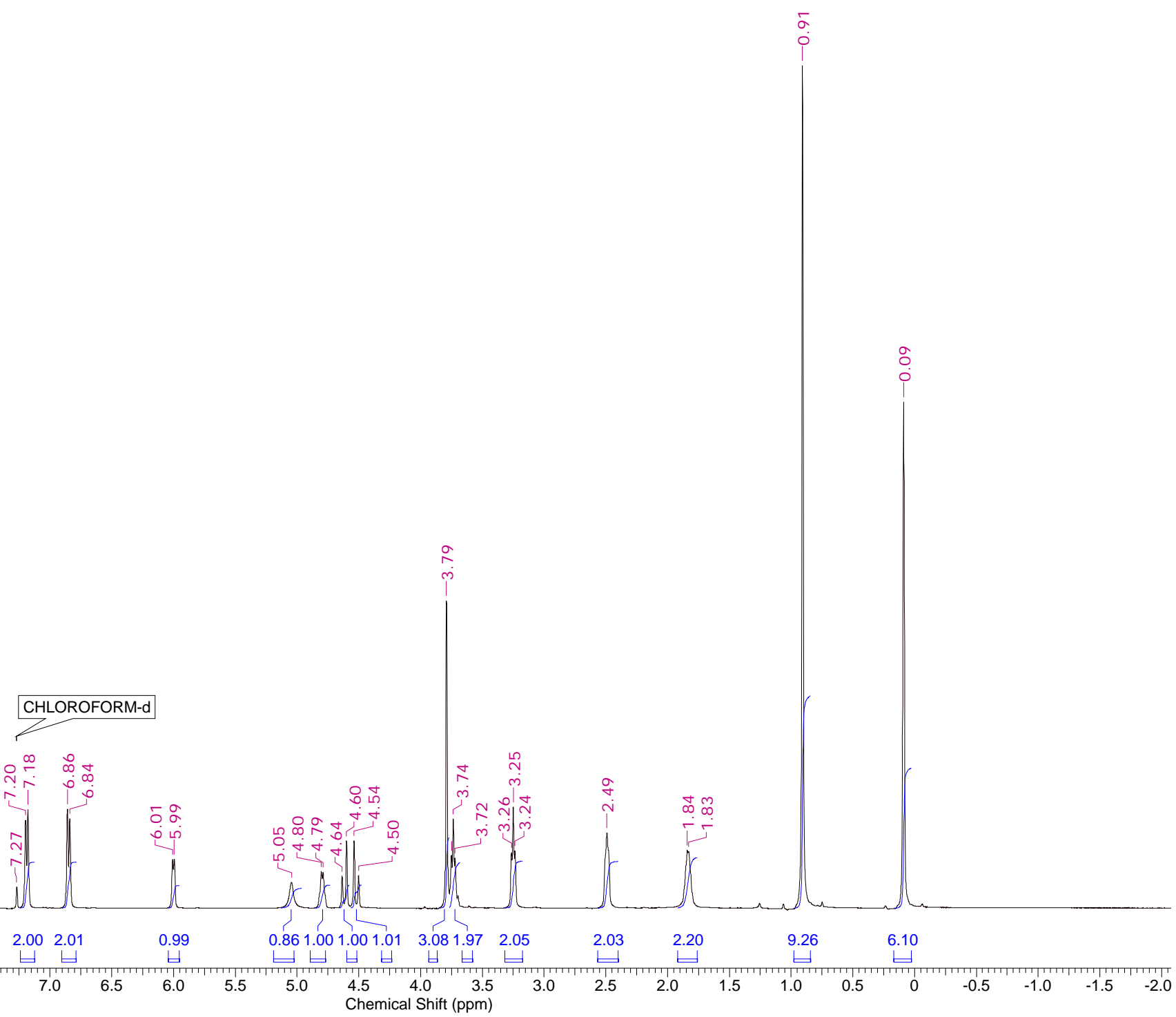




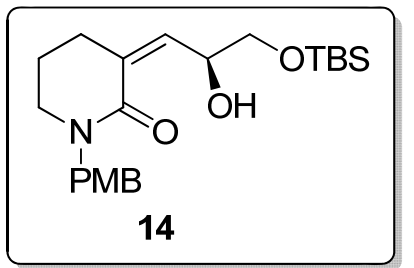

${ }^{13} \mathrm{C}-\mathrm{NMR}\left(101 \mathrm{MHz}, \mathrm{CDCl}_{3}\right)$

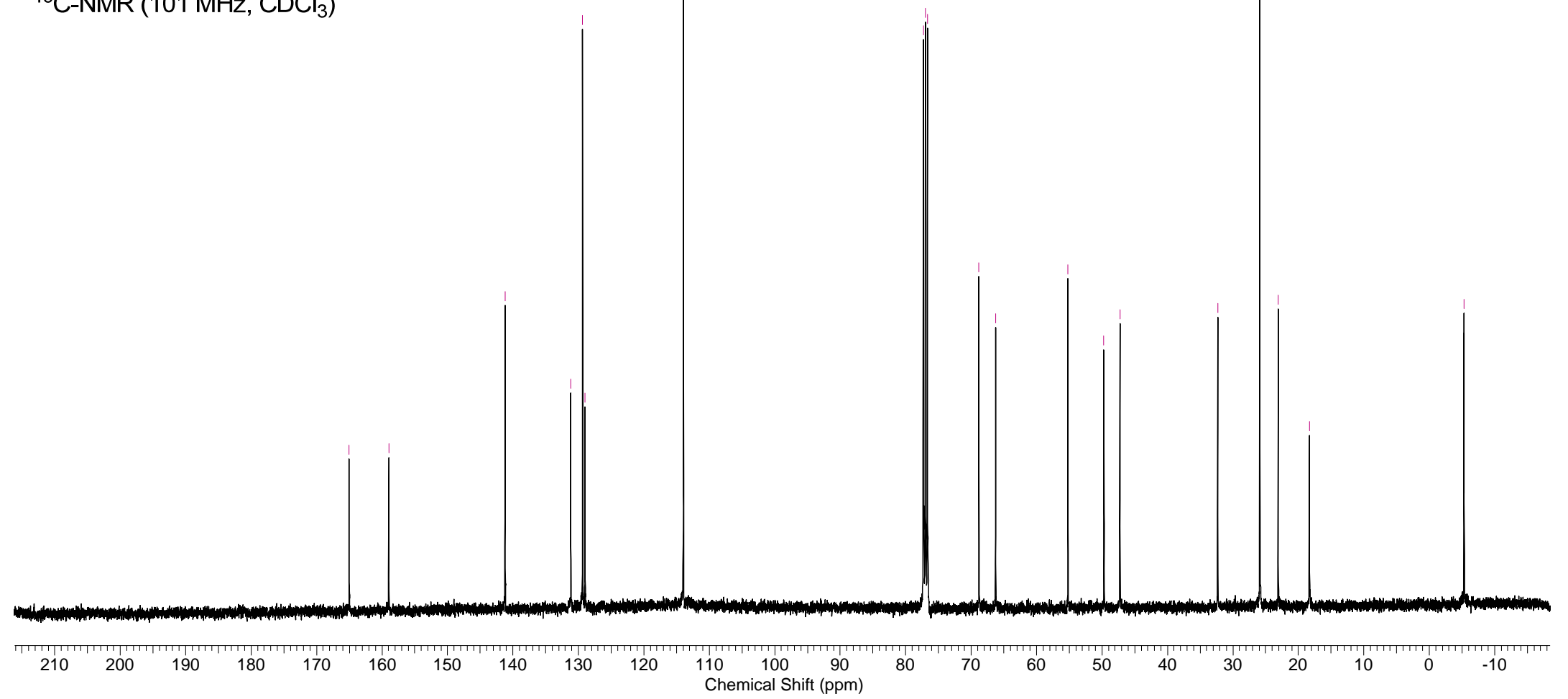


SKB PMBTBS-2.003.001.1R.ESP

CHLOROFORM-d

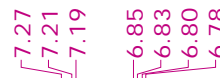

${ }^{1} \mathrm{H}-\mathrm{NMR}\left(400 \mathrm{MHz}, \mathrm{CDCl}_{3}\right)$

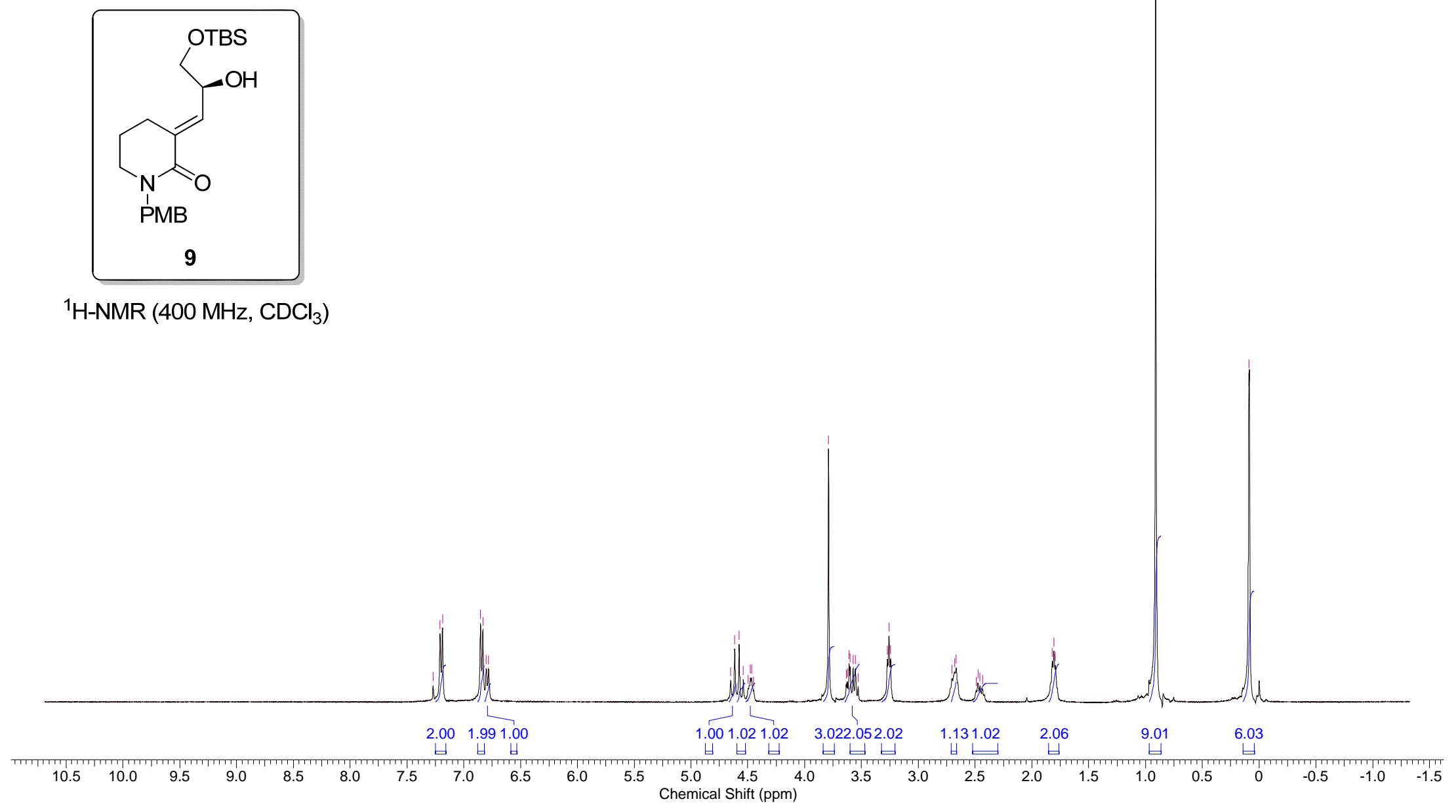

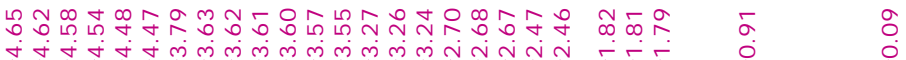




\begin{tabular}{|c|c|c|c|c|c|}
\hline 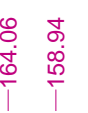 & 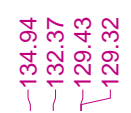 & 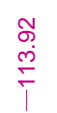 & 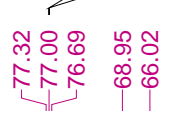 & 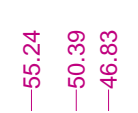 & 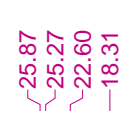 \\
\hline
\end{tabular}

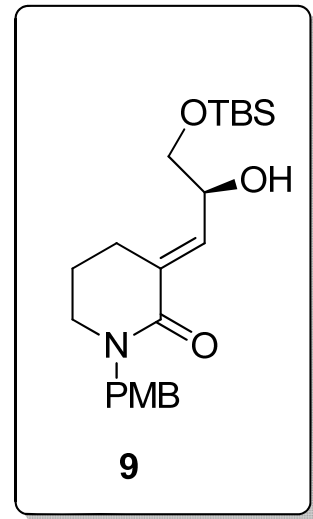

${ }^{13} \mathrm{C}-\mathrm{NMR}\left(101 \mathrm{MHz}, \mathrm{CDCl}_{3}\right)$

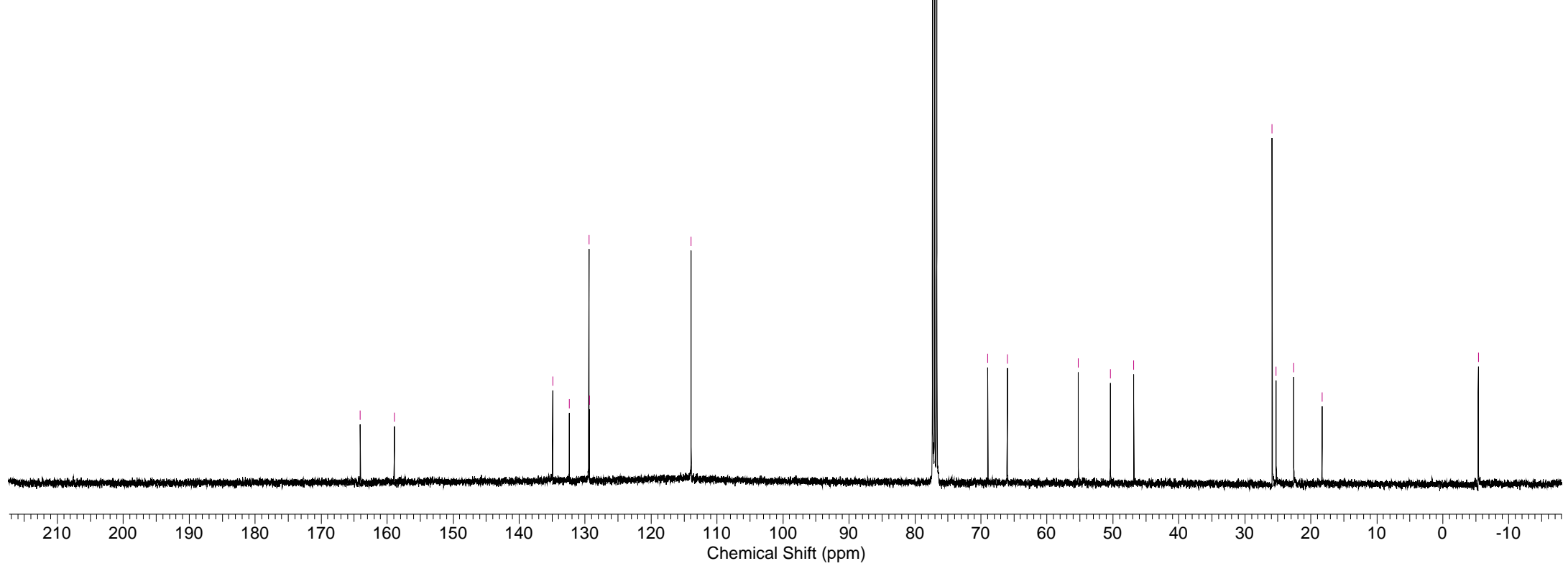




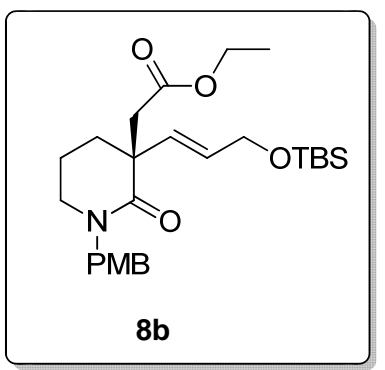

${ }^{1} \mathrm{H}-\mathrm{NMR}\left(400 \mathrm{MHz}, \mathrm{CDCl}_{3}\right)$

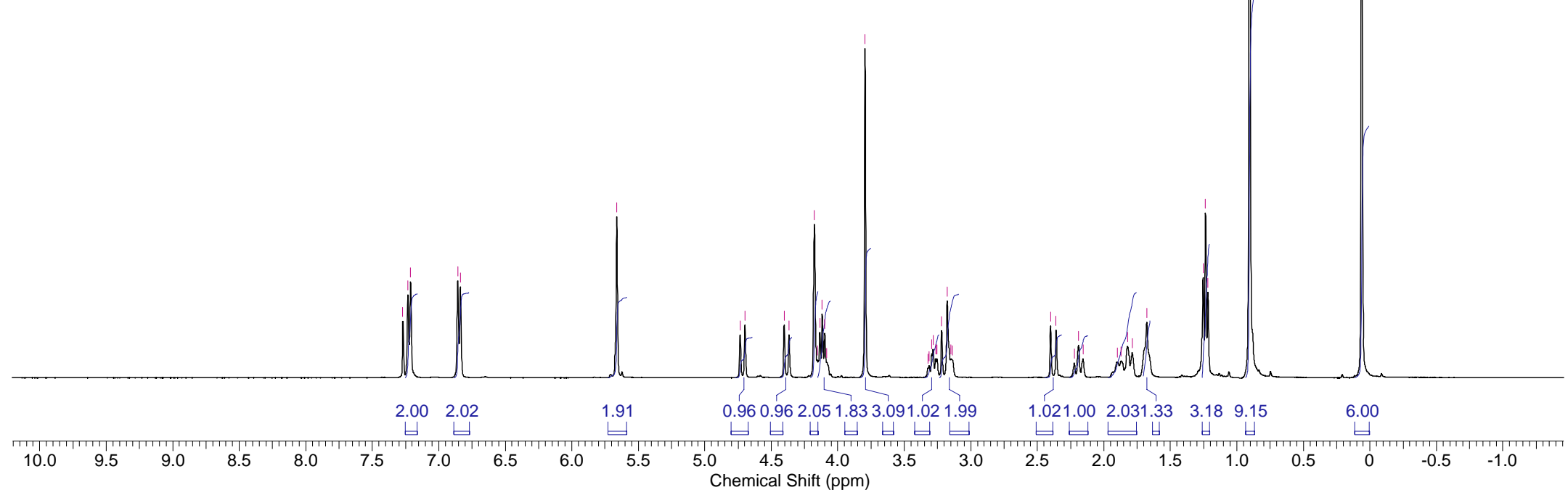




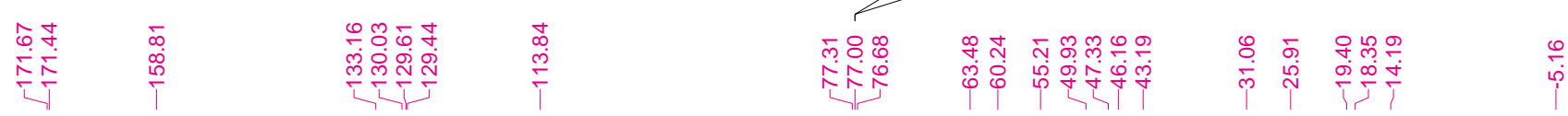

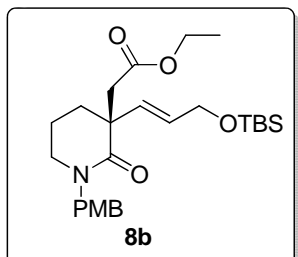

${ }^{13} \mathrm{C}-\mathrm{NMR}\left(101 \mathrm{MHz}, \mathrm{CDCl}_{3}\right)$

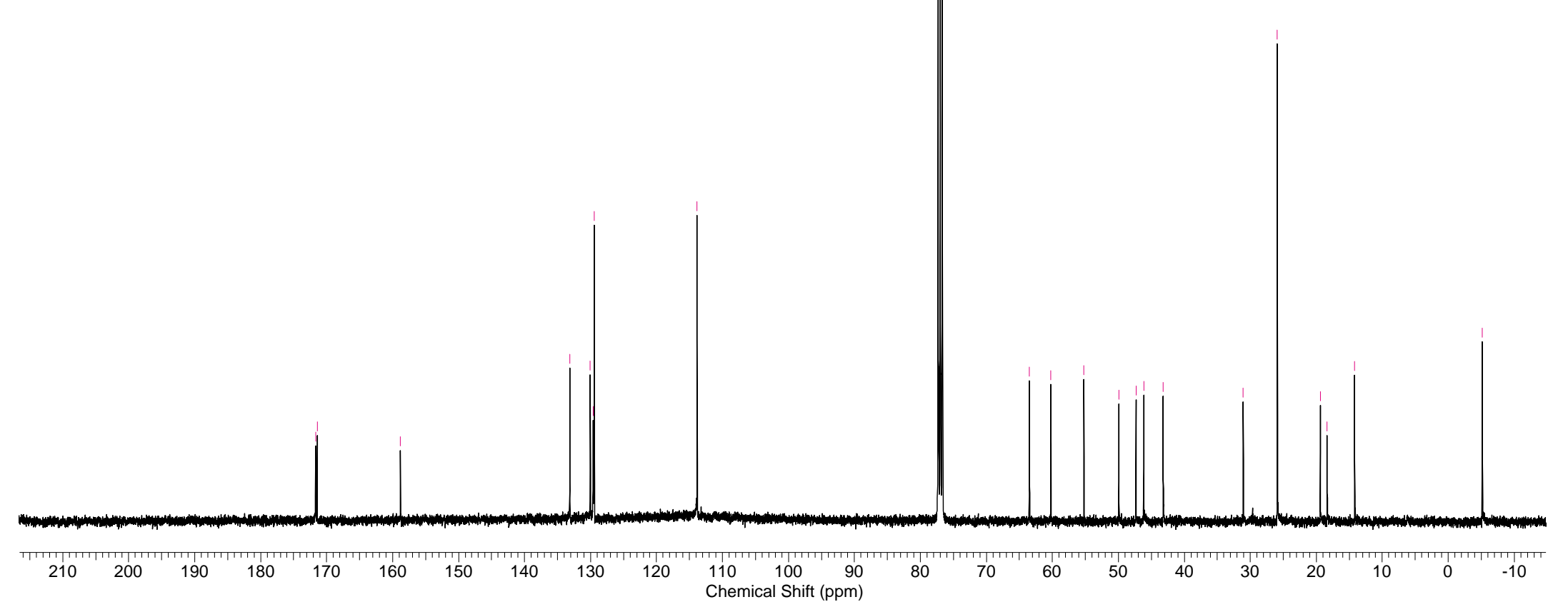




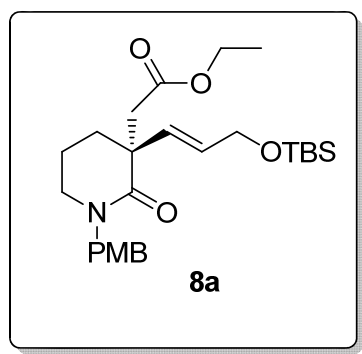

${ }^{1} \mathrm{H}-\mathrm{NMR}\left(400 \mathrm{MHz}, \mathrm{CDCl}_{3}\right)$

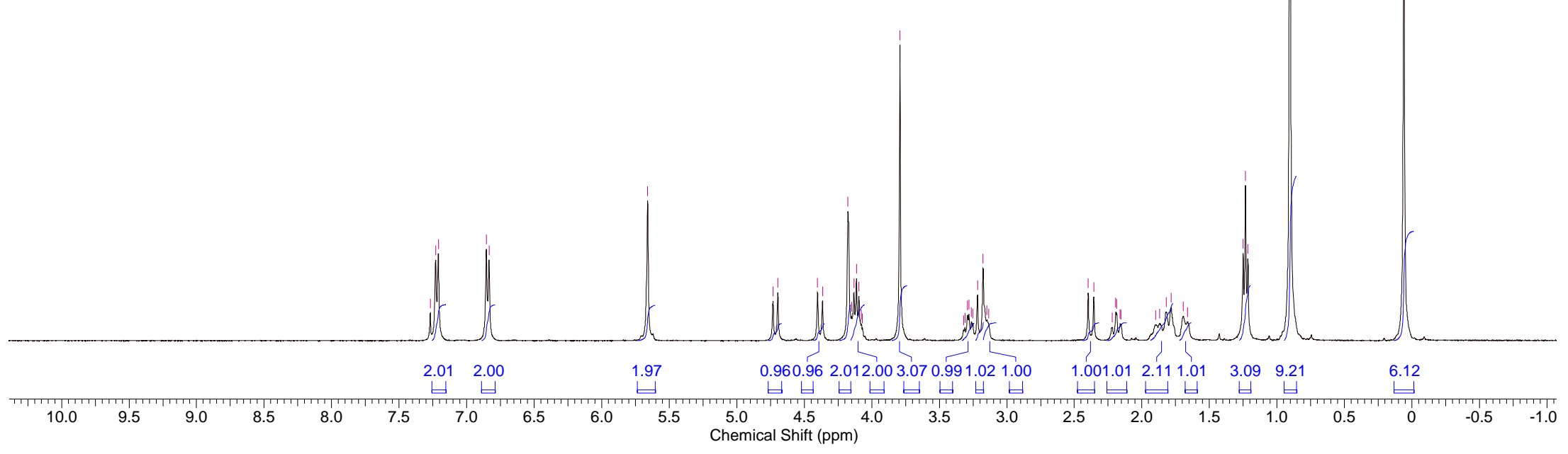


skb 02 158_c1.004_001_1r.esp

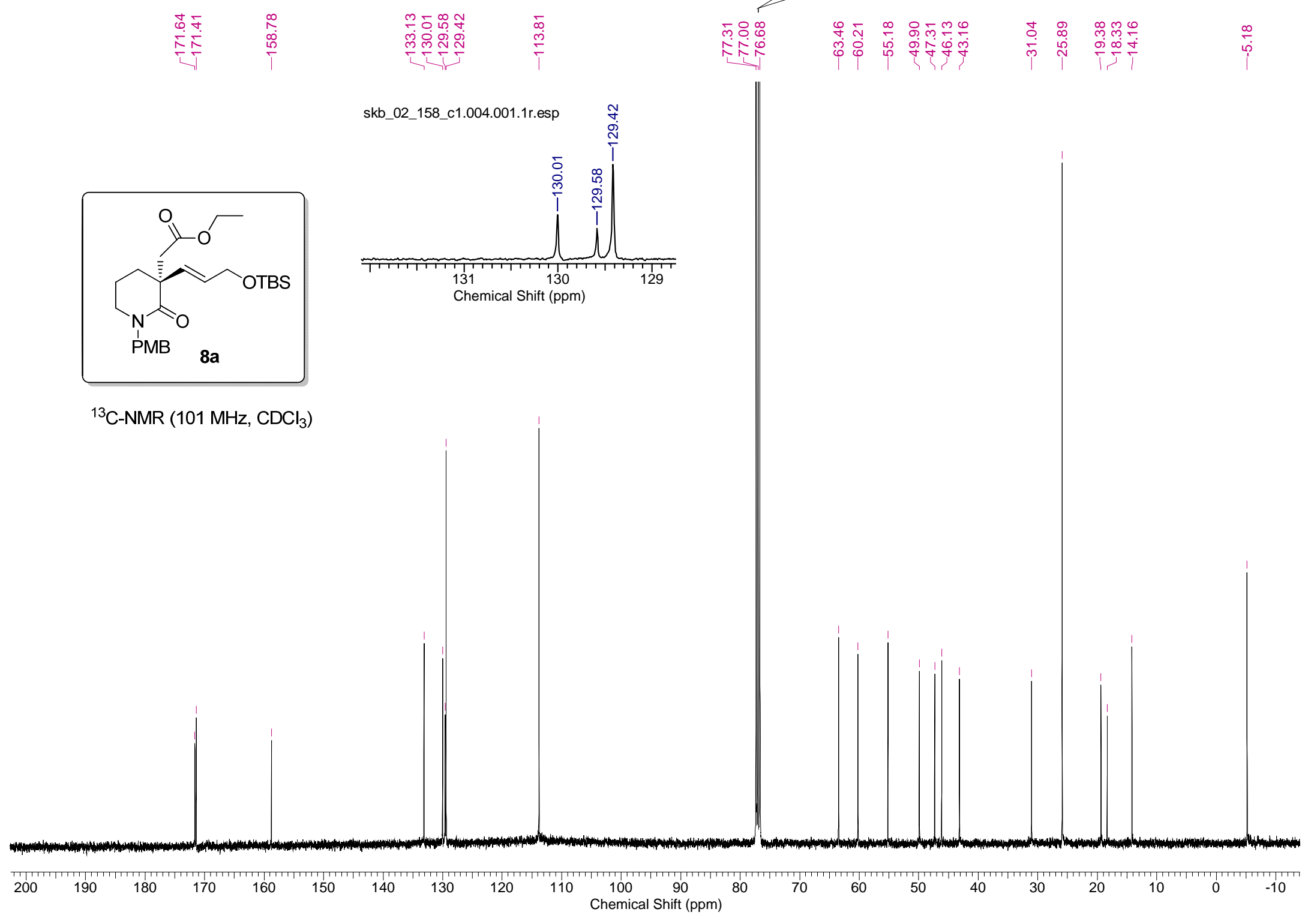




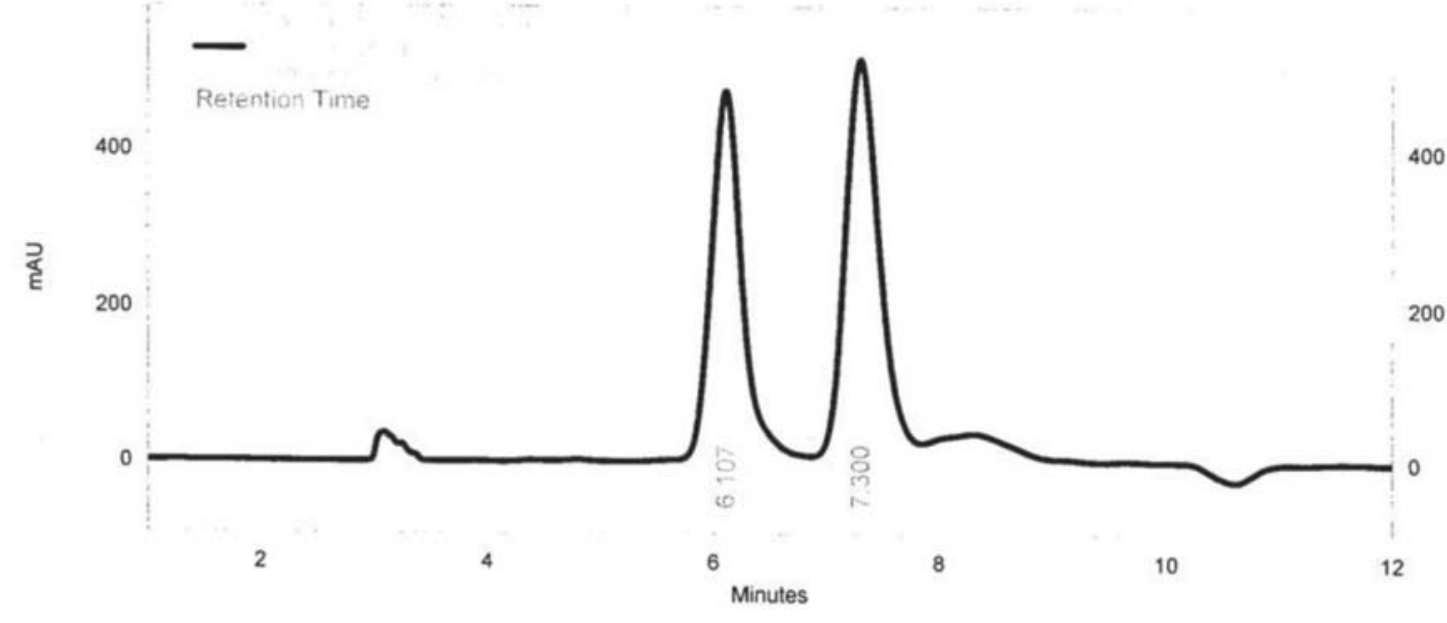

400

DAD: Signal C,

$210 \mathrm{~nm} / \mathrm{Bw}: 4 \mathrm{~nm}$

Results

\begin{tabular}{rrrrrr|} 
Retention Time & Area & Area \% & Height & Height \% \\
\hline 6.107 & 18526906 & 46.47 & 988626 & 48.45 \\
7.300 & 21338234 & 53.53 & 1052056 & 51.55 \\
\hline \multicolumn{2}{|c|}{ Totals } & 39865140 & 100.00 & 2040682 & 100.00 \\
\hline
\end{tabular}

Column: CHIRALCELOD-H

Solvent: Hexane:Isopropanol (90:10)

Wavelength-210nm

Flow Rate- $1 \mathrm{ML} / \mathrm{min}$

Pressure: $43 \mathrm{bar}$

Operator :SHIVA 


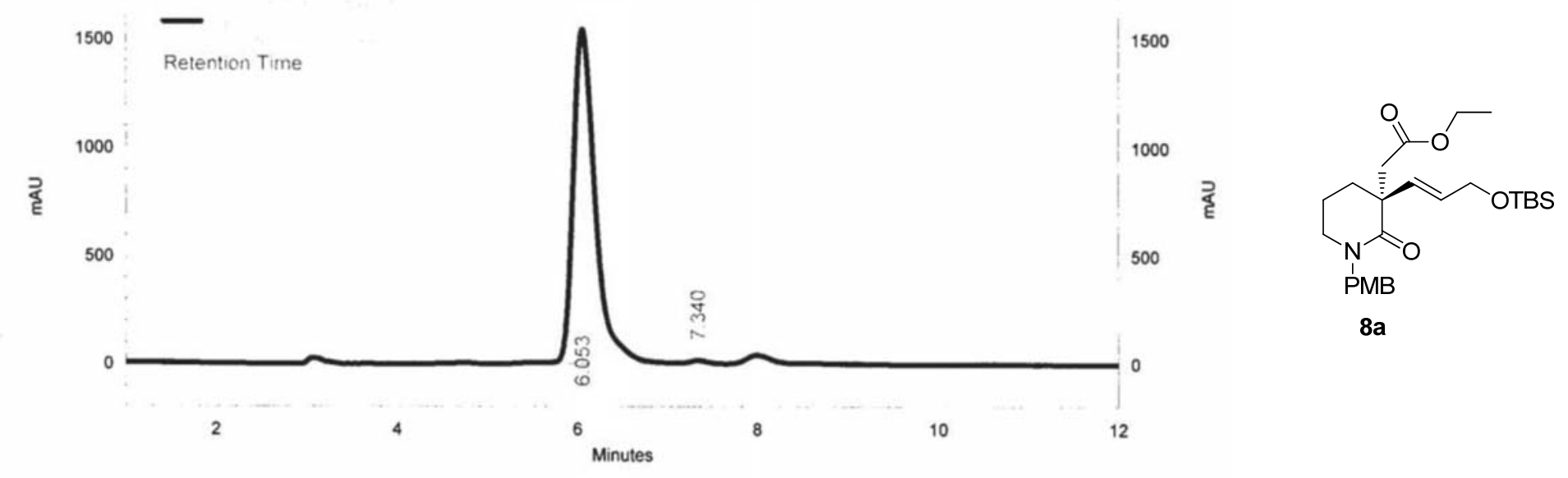

DAD: Signal C,

$210 \mathrm{~nm} / \mathrm{Bw}: 4 \mathrm{~nm}$

Results

\begin{tabular}{rrrrrr|} 
Retention Time & Area & Area \% & Height & Height \% \\
\hline 6.053 & 55266628 & 99.80 & 3236296 & 99.54 \\
7.340 & 110081 & 0.20 & 14921 & 0.46 \\
\hline \multicolumn{7}{|c|}{ Totals } & 55376709 & 100.00 & 3251217 & 100.00 \\
\hline
\end{tabular}

Column: CHIRALCELOD-H

Solvent: Hexane:Isopropanol (90:10)

Wavelength-210nm

Flow Rate-1ML/min

Pressure: 43 bar

Operator :SHIVA 


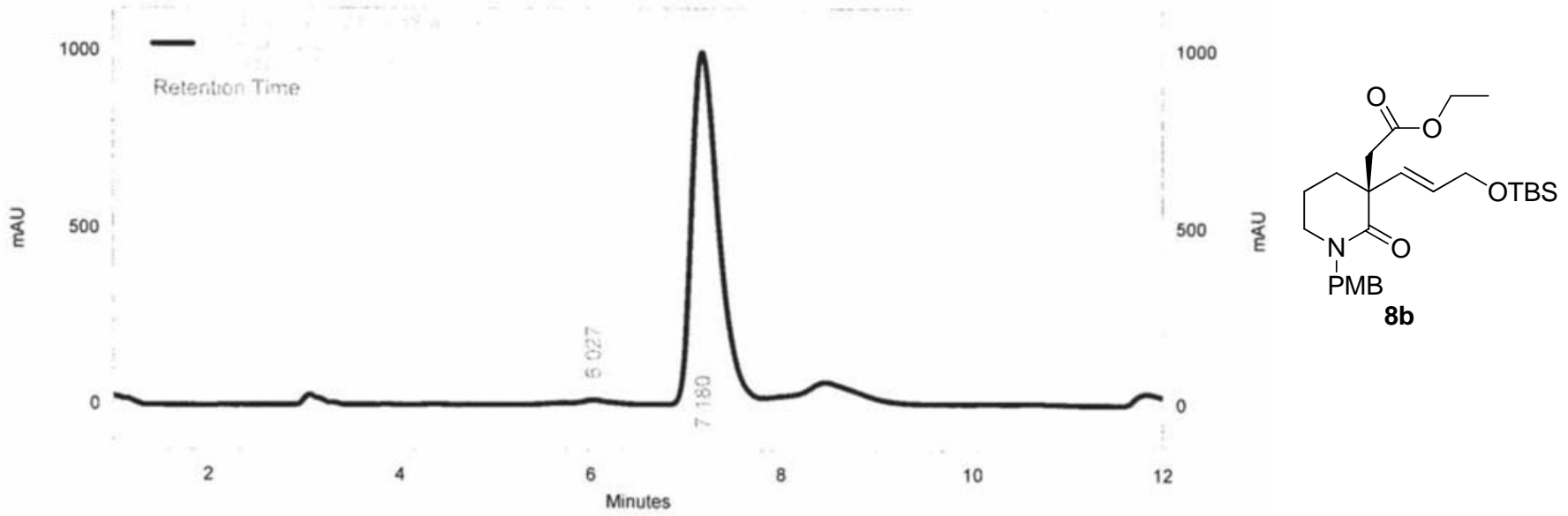

DAD: Signal C,

$210 \mathrm{~nm} / \mathrm{Bw}: 4 \mathrm{~nm}$

Results

\begin{tabular}{rrrrrr|} 
Retention Time & Area & Area \% & Height & Height \% \\
\hline 6.027 & 72404 & 0.18 & 8120 & 0.39 \\
7.180 & 39846816 & 99.82 & 2074459 & 99.61 \\
\hline Totals & 39919220 & 100.00 & 2082579 & 100.00 \\
\hline
\end{tabular}

Column: CHIRALCELOD-H

Solvent: Hexane:Isopropanol (90:10)

Wavelength-210nm

Flow Rate-1 $\mathrm{ML} / \mathrm{min}$

Pressure: 43 bar

Operator:SHIVA 
1H 149-PMB-ALD.001.001.1R.ESP

$\stackrel{\stackrel{n}{\infty}}{\stackrel{\infty}{\infty}}$

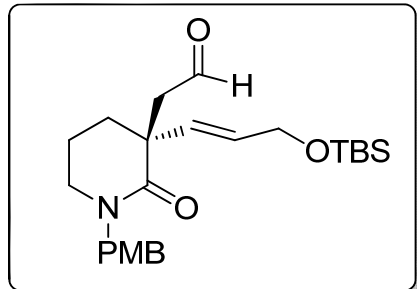

${ }^{1} \mathrm{H}-\mathrm{NMR}\left(400 \mathrm{MHz}, \mathrm{CDCl}_{3}\right)$
CHLOROFORM-d

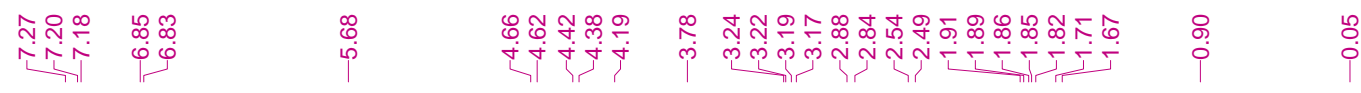

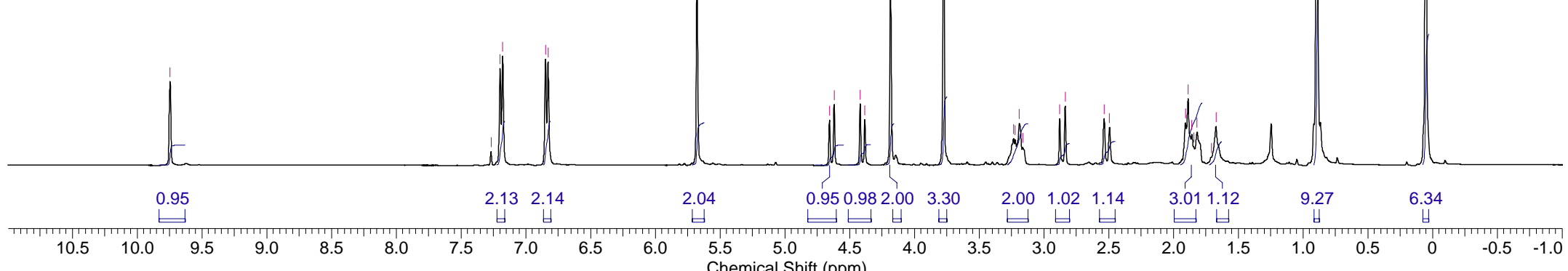


13C 149-PMB-ALD.002.001.1R.ESP

$$
\text { OTBS }
$$

${ }^{13} \mathrm{C}-\mathrm{NMR}\left(101 \mathrm{MHz}, \mathrm{CDCl}_{3}\right)$

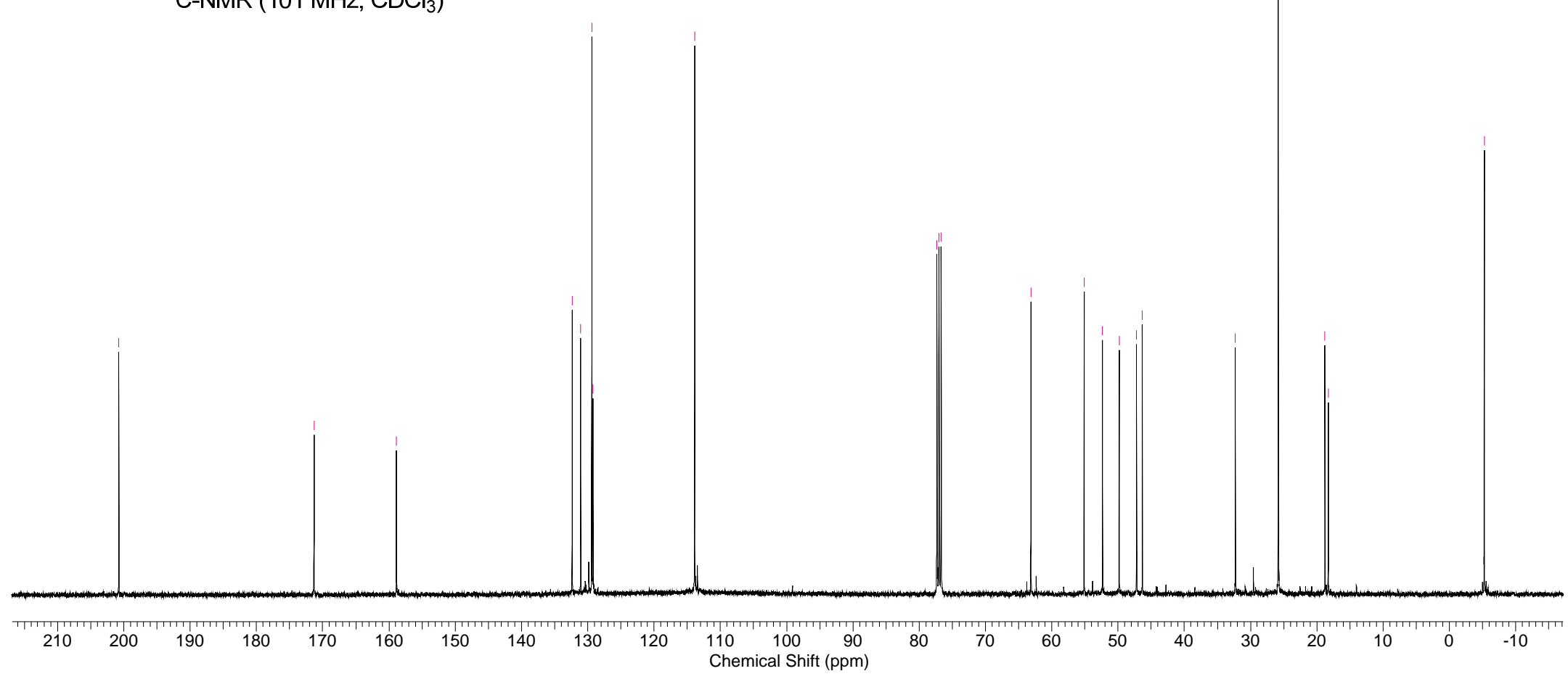


skb_pmb ald 1.002.001.1r.esp

$\stackrel{n}{\stackrel{n}{i}}$

${ }^{1} \mathrm{H}-\mathrm{NMR}\left(400 \mathrm{MHz}, \mathrm{CDCl}_{3}\right)$

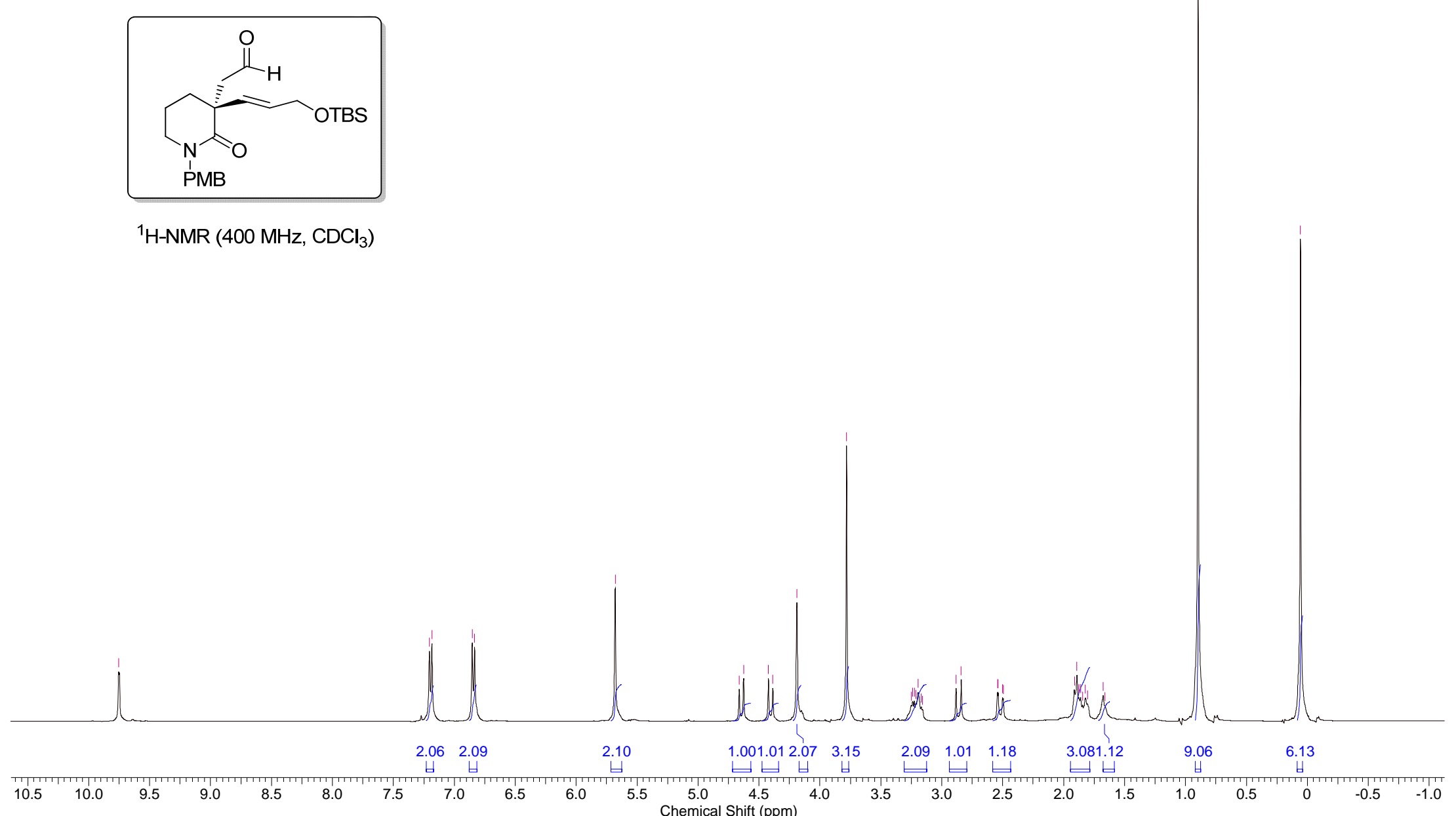

CHLOROFORM-d

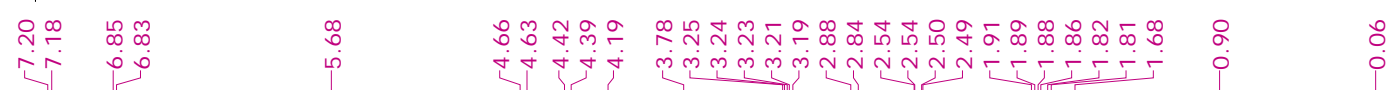




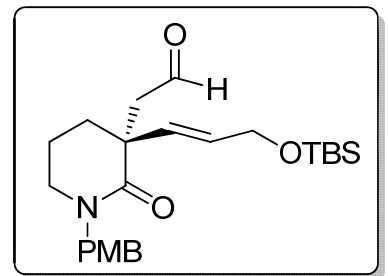

${ }^{13} \mathrm{C}-\mathrm{NMR}\left(101 \mathrm{MHz}, \mathrm{CDCl}_{3}\right.$ )

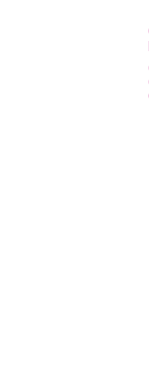

ণ
¿
ஸे

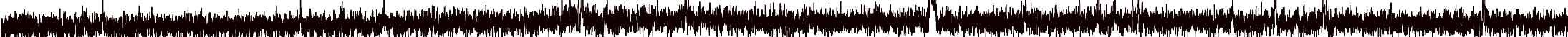

CHLOROFORM-d

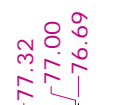


1H_DITHIANE_2.003.00EHARGHEDFORM-d

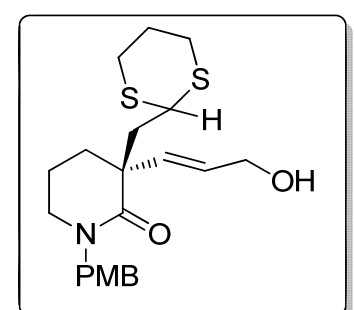

${ }^{1} \mathrm{H}-\mathrm{NMR}\left(400 \mathrm{MHz}, \mathrm{CDCl}_{3}\right)$

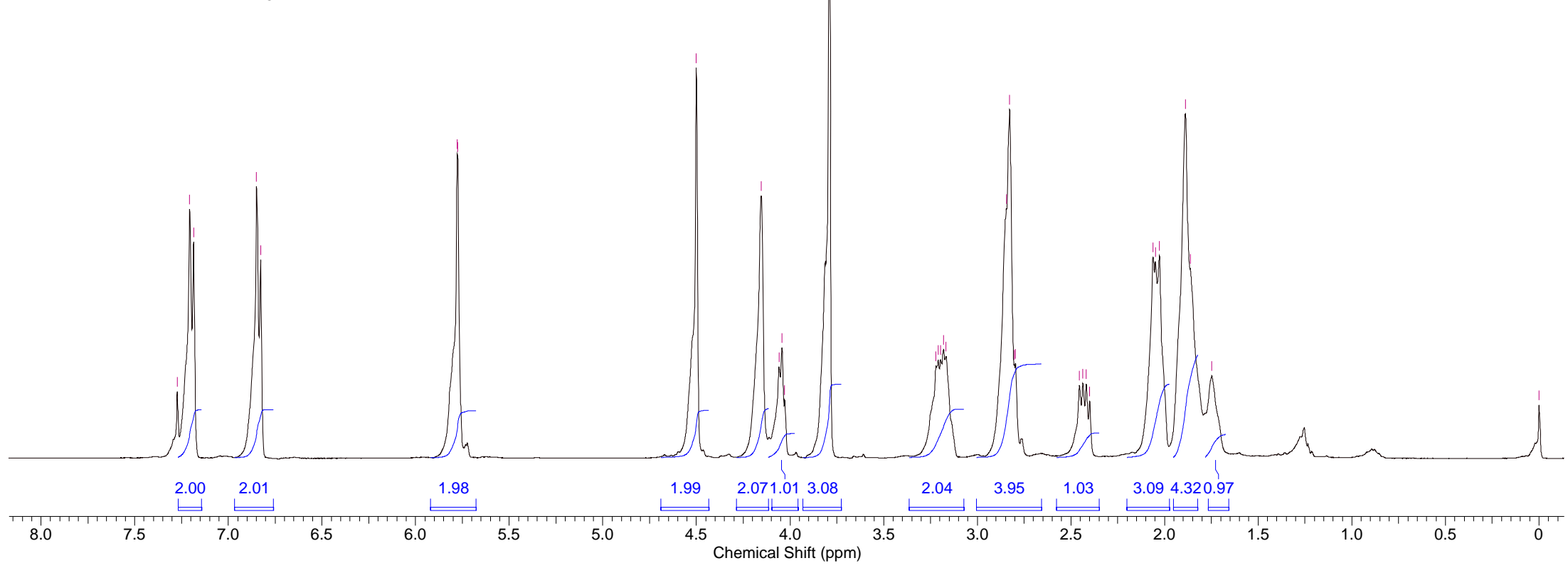




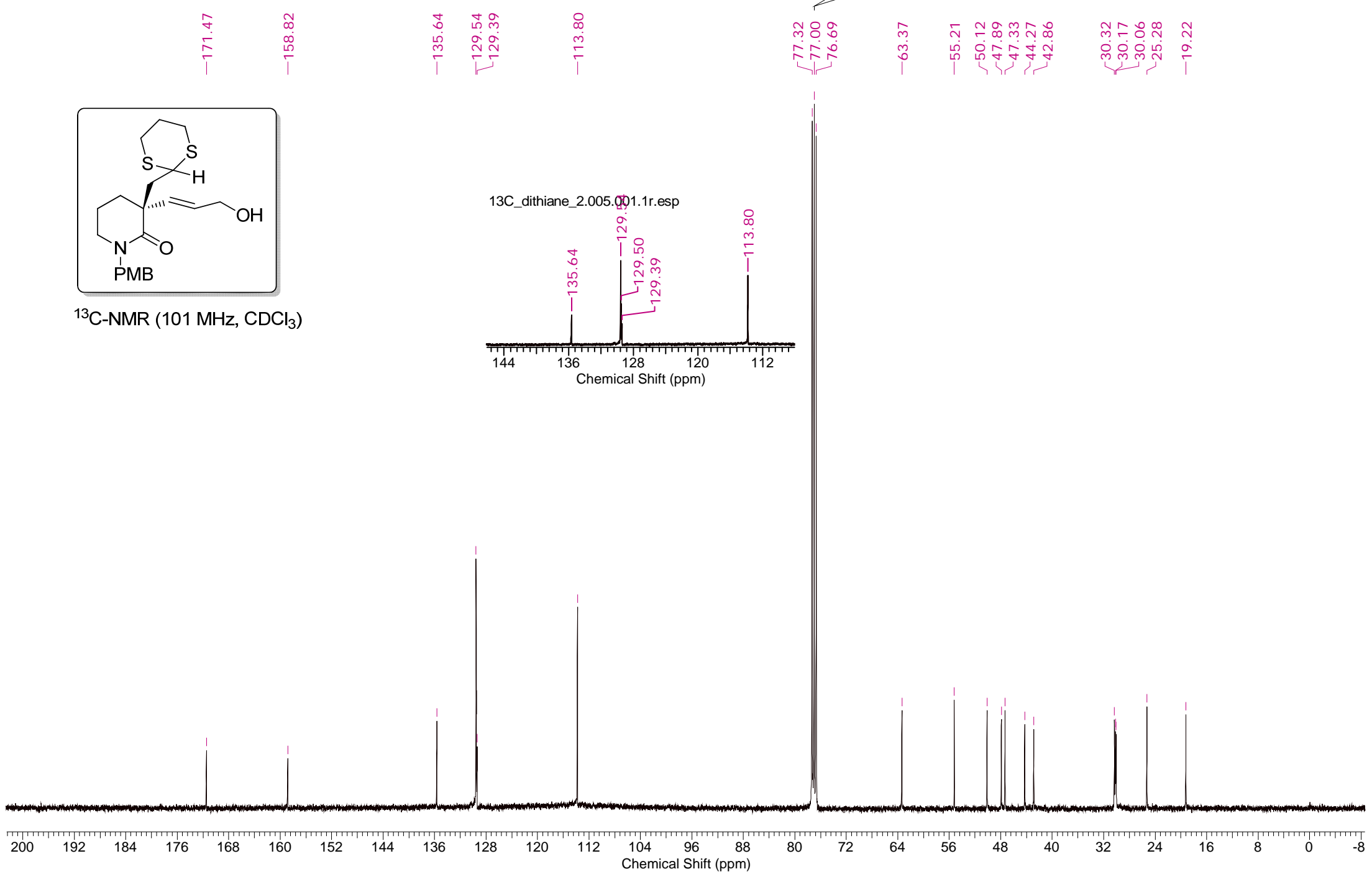




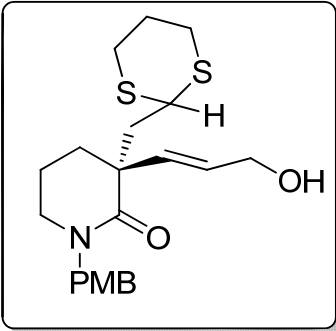

${ }^{1} \mathrm{H} \mathrm{NMR},\left(400 \mathrm{MHz}, \mathrm{CDCl}_{3}\right)$

$2.05 \quad 2.02$

2.00

1.982 .071 .053 .02

$2.29 \quad 4.111 .141 .423 .023 .251 .13$

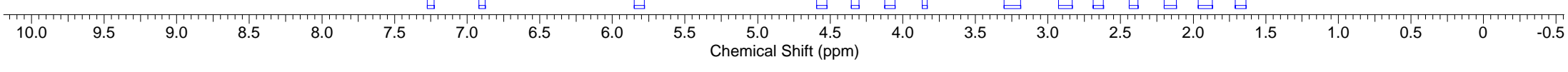




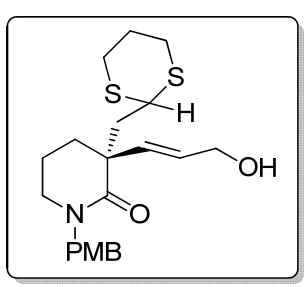

$\left.{ }^{13} \mathrm{C} \mathrm{NMR,(101} \mathrm{MHz,CDCl}\right)_{3}$

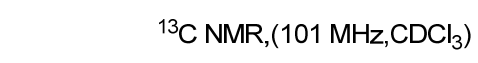

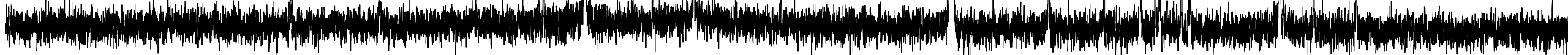

200
$180 \quad 170$

160

$150 \quad 140$

$40 \quad 130$

120

$110 \quad 100 \quad 90$

80

70

50

40

30

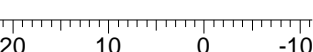




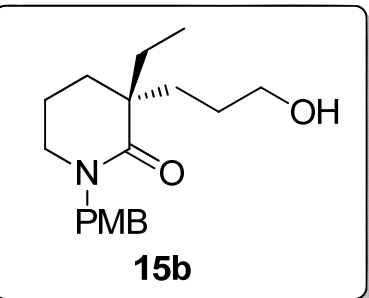

${ }^{1} \mathrm{H}-\mathrm{NMR}\left(800 \mathrm{MHz}, \mathrm{CDCl}_{3}\right)$

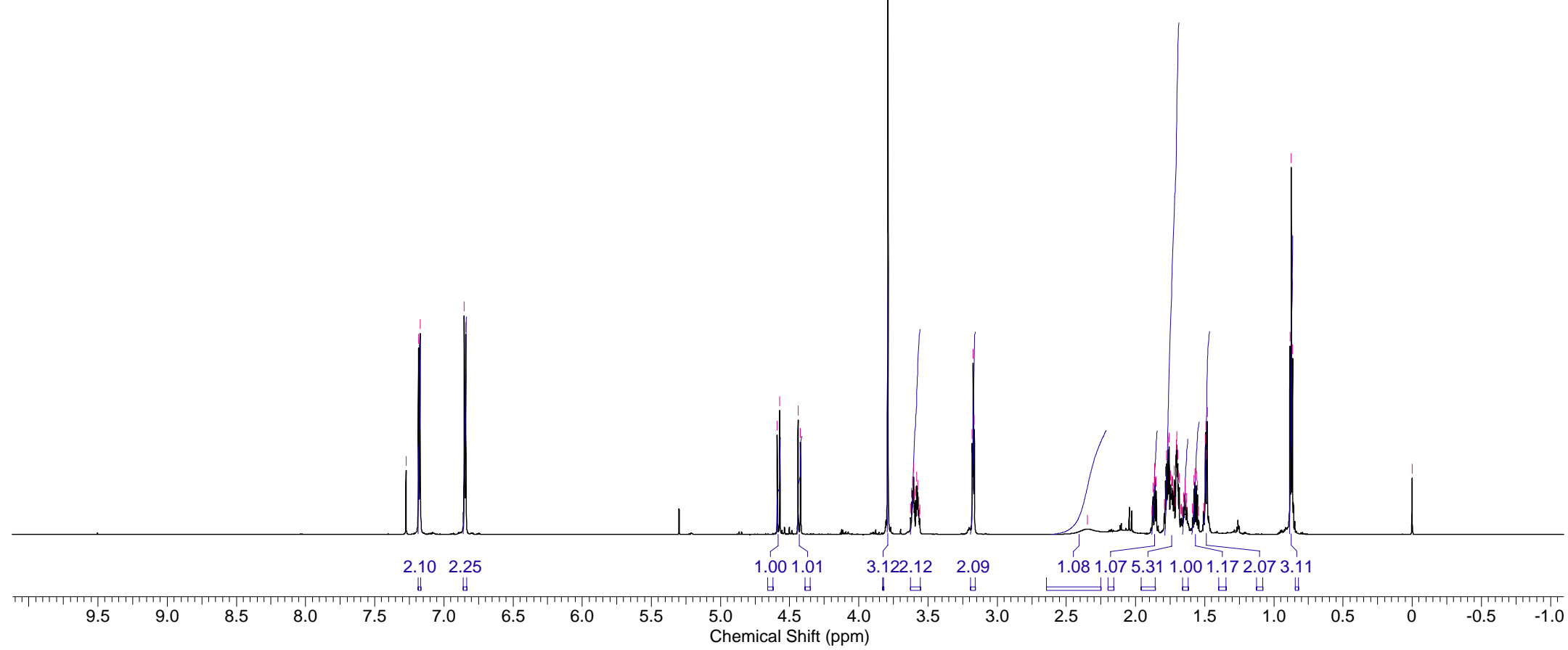




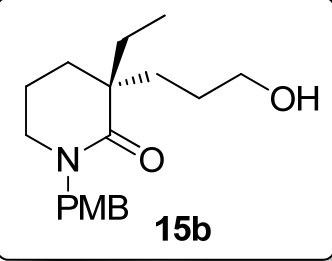

${ }^{13} \mathrm{C}-\mathrm{NMR}\left(101 \mathrm{MHz}, \mathrm{CDCl}_{3}\right)$

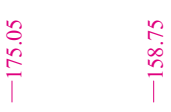

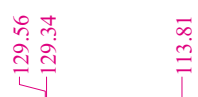

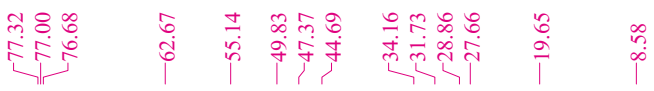

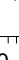

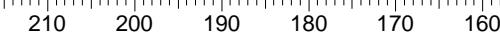

$150 \quad 140$

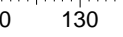

120

$\begin{array}{ccc}110 & 100 & 90 \\ \text { Chemical Shift } & 90\end{array}$

$80 \quad 70$

$50 \quad 40$

30

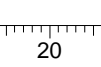

10

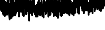




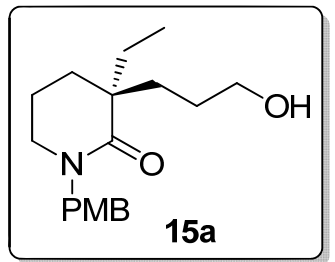

${ }^{1} \mathrm{H}-\mathrm{NMR}\left(400 \mathrm{MHz}, \mathrm{CDCl}_{3}\right.$ )

CHLOROFORM-d

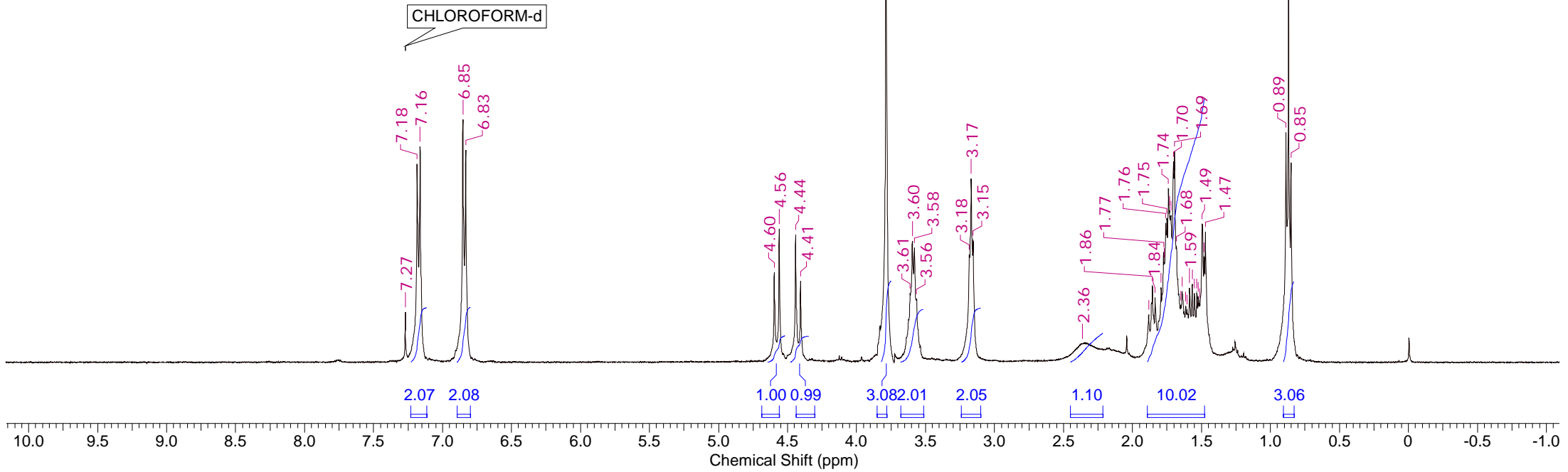




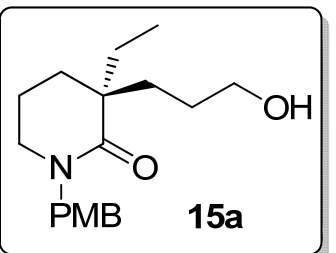

${ }^{13} \mathrm{C}-\mathrm{NMR}\left(101 \mathrm{MHz}, \mathrm{CDCl}_{3}\right.$ )

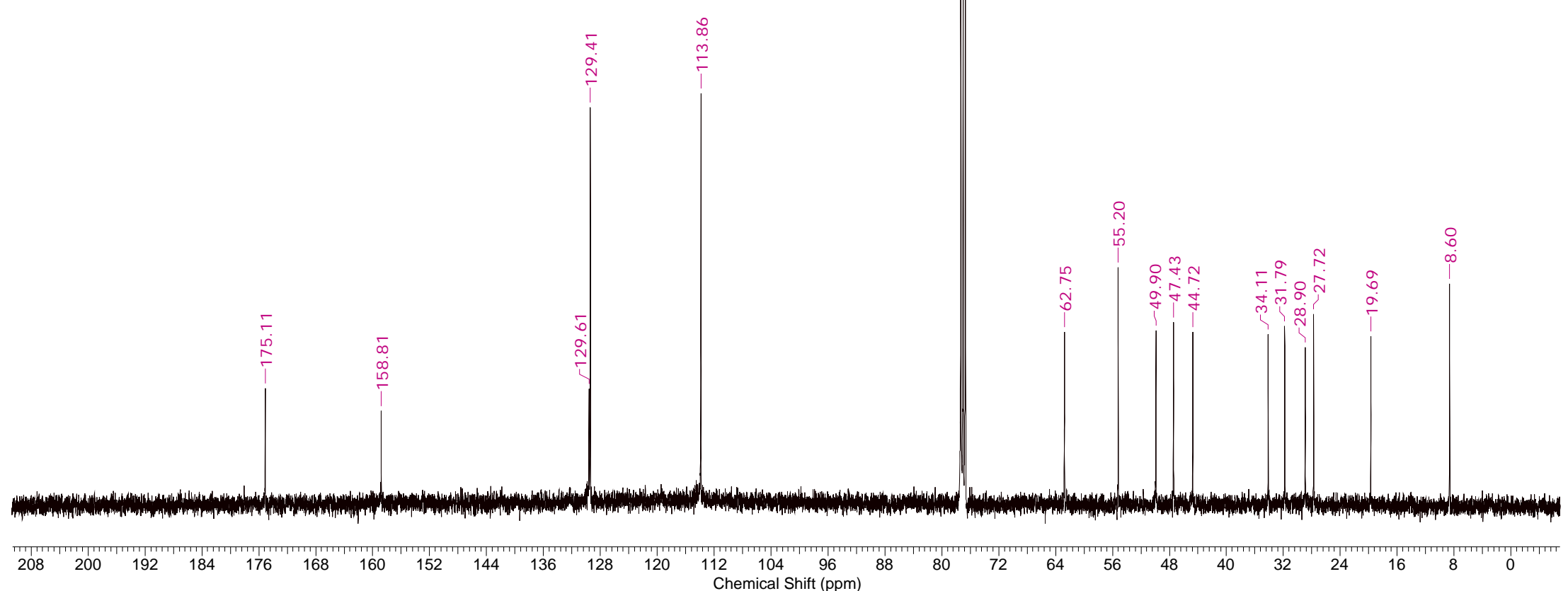




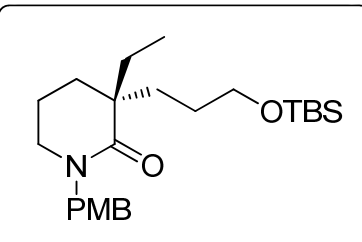

${ }^{1} \mathrm{H}-\mathrm{NMR}\left(800 \mathrm{MHz}, \mathrm{CDCl}_{3}\right)$

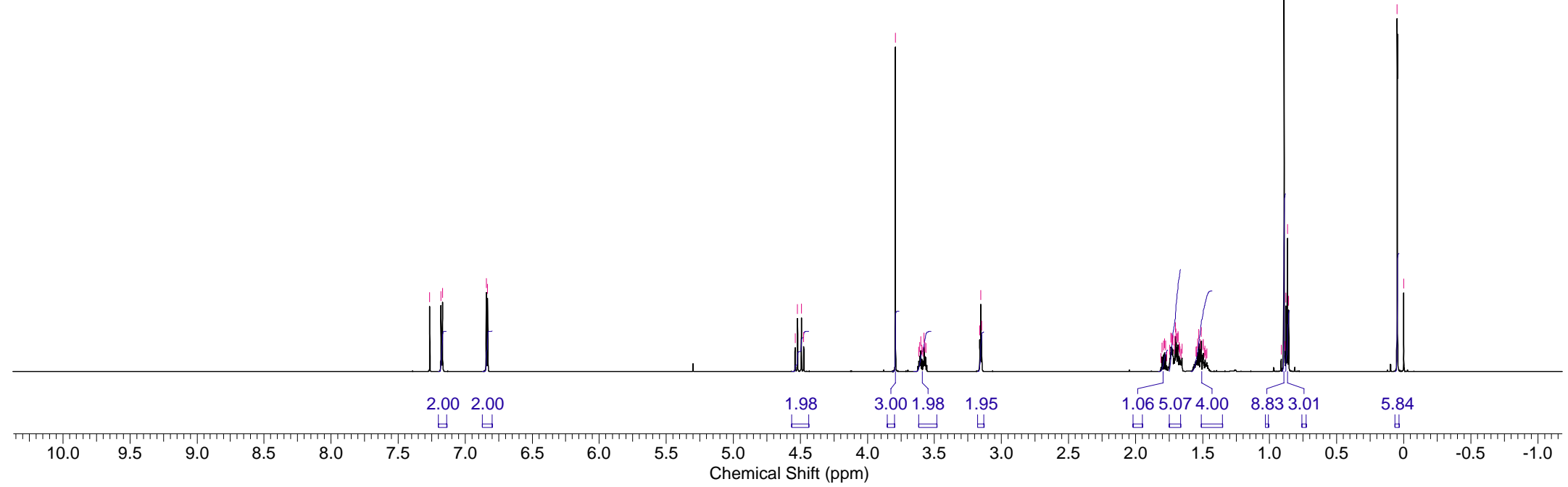


SKB_PMB_ETOTBS.009.001.1R.ESP

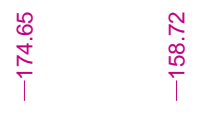$$
\text { OTBS }
$$

${ }^{13} \mathrm{C}-\mathrm{NMR}\left(101 \mathrm{MHz}, \mathrm{CDCl}_{3}\right)$

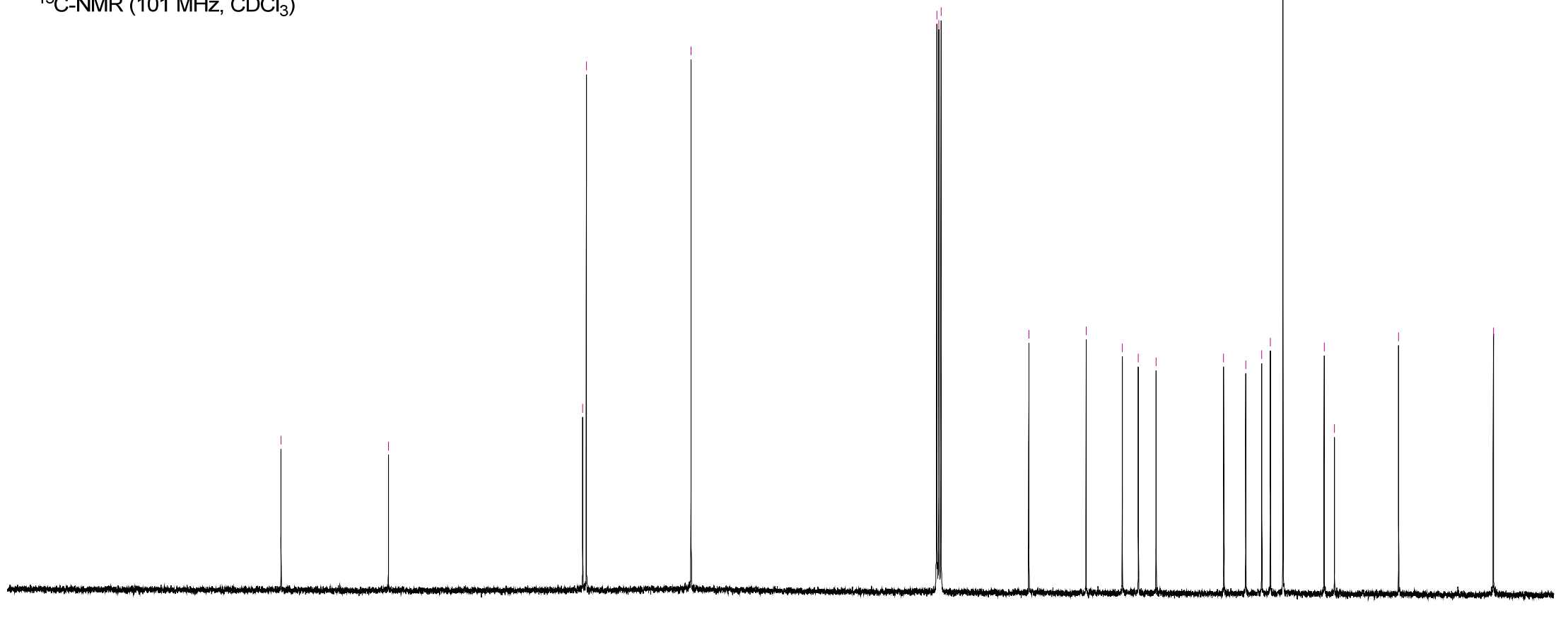

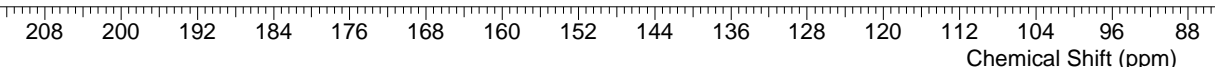




\begin{tabular}{|c|}
\hline 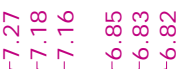 \\
\hline
\end{tabular}

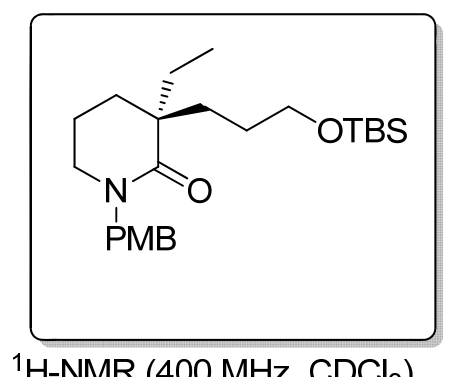

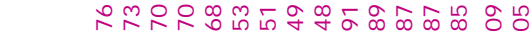

${ }^{1} \mathrm{H}-\mathrm{NMR}\left(400 \mathrm{MHz}, \mathrm{CDCl}_{3}\right)$

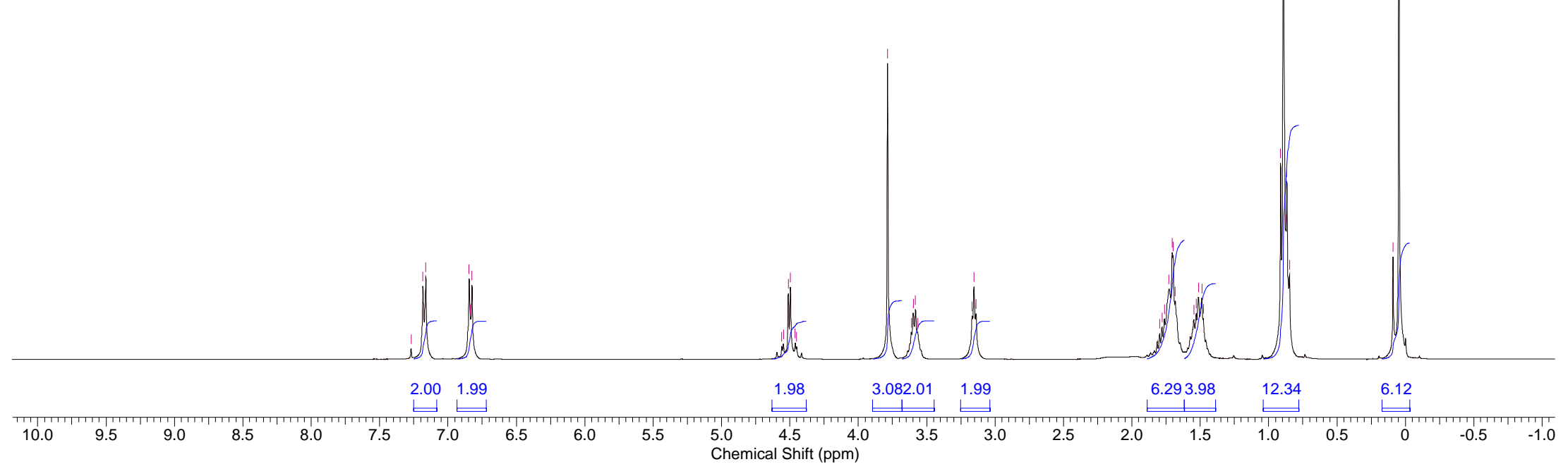



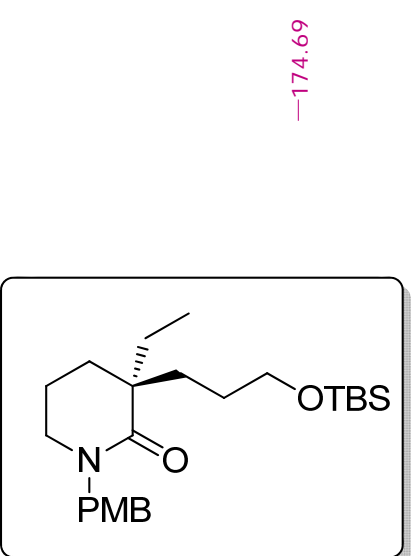

${ }^{13} \mathrm{C}-\mathrm{NMR}\left(101 \mathrm{MHz}, \mathrm{CDCl}_{3}\right.$ )

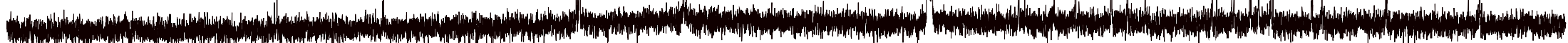

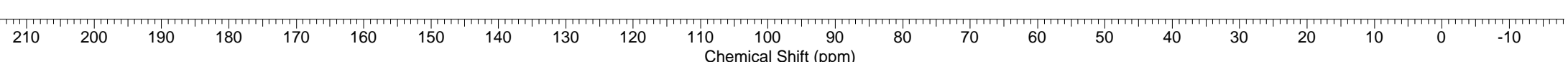




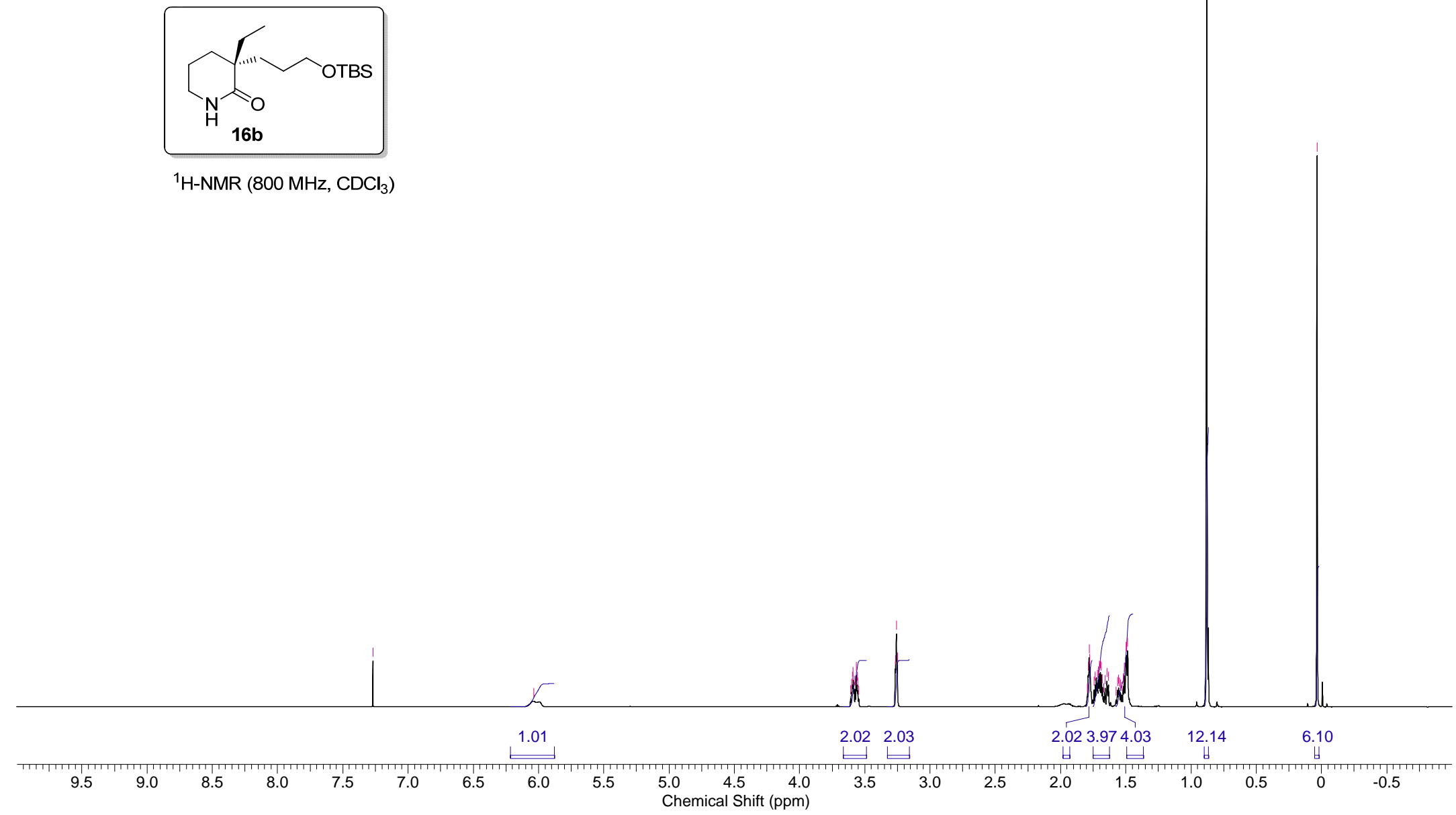

${ }^{1} \mathrm{H}-\mathrm{NMR}\left(800 \mathrm{MHz}, \mathrm{CDCl}_{3}\right)$ 


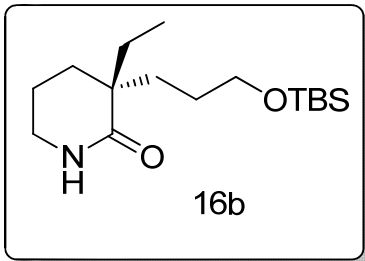

${ }^{13} \mathrm{C}-\mathrm{NMR}\left(101 \mathrm{MHz}, \mathrm{CDCl}_{3}\right)$

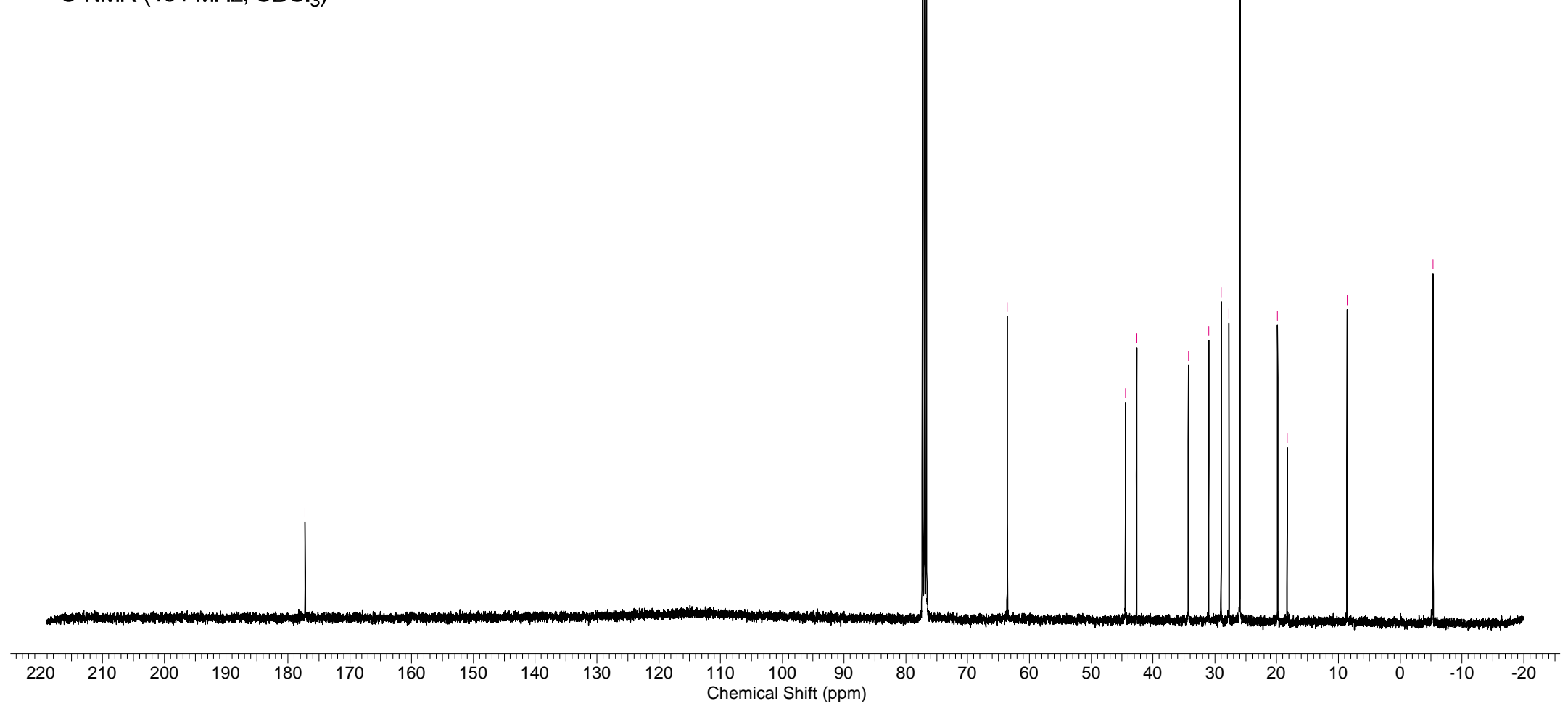




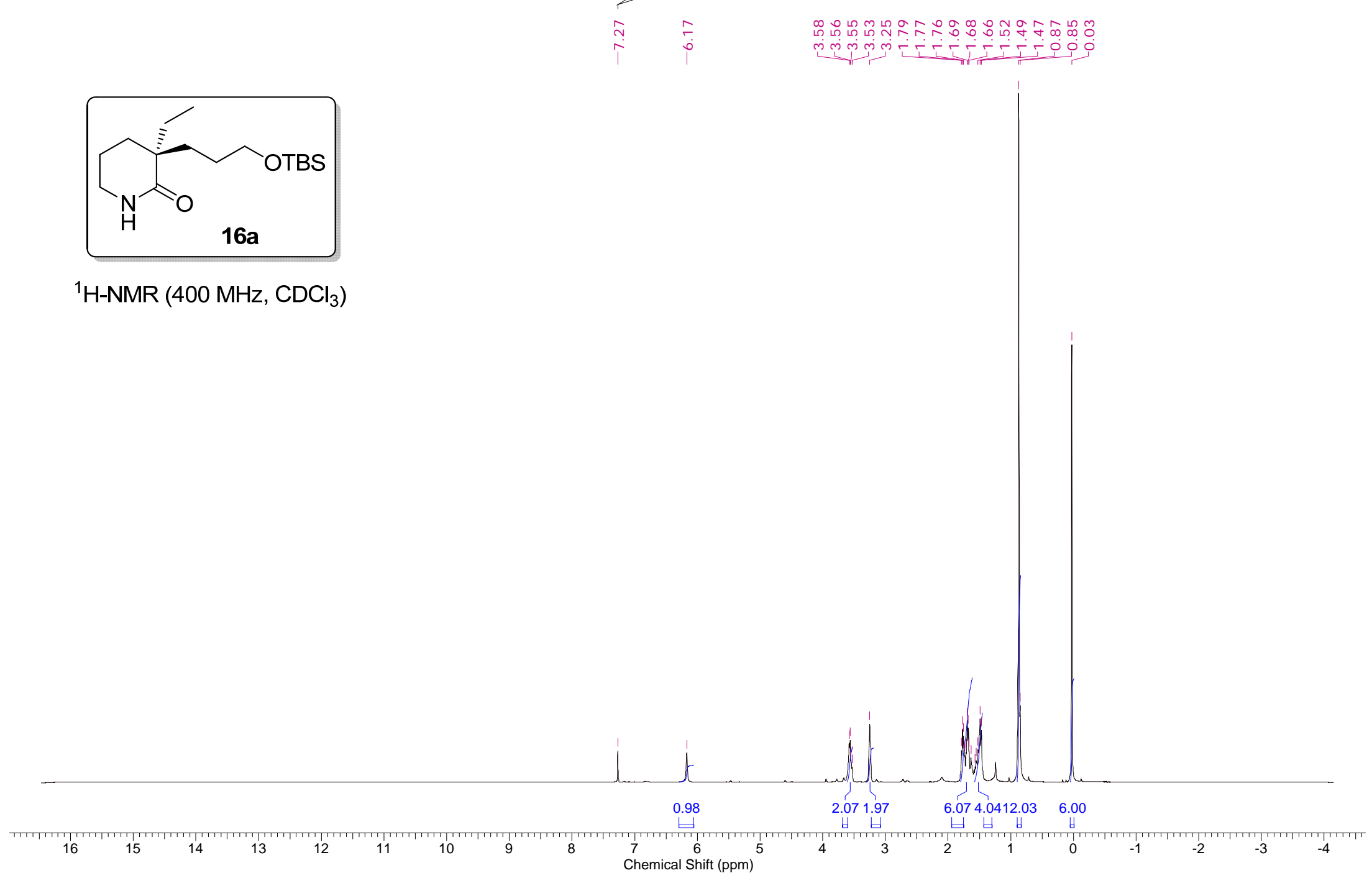


skb_02_139-lactam-otbs.004.001.1r.esp

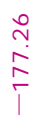

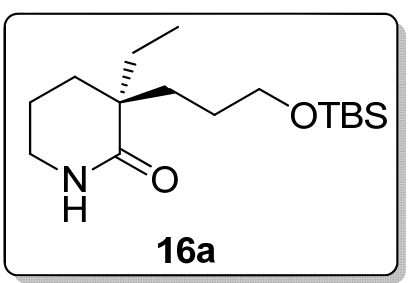

${ }^{13} \mathrm{C}-\mathrm{NMR}\left(101 \mathrm{MHz}, \mathrm{CDCl}_{3}\right)$
CHLOROFORM-d

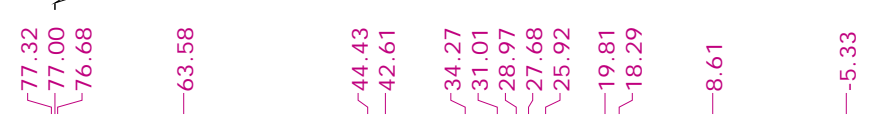

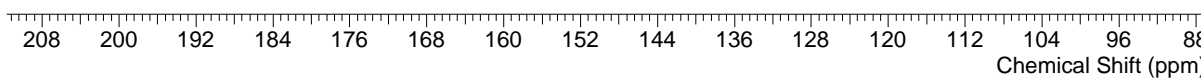




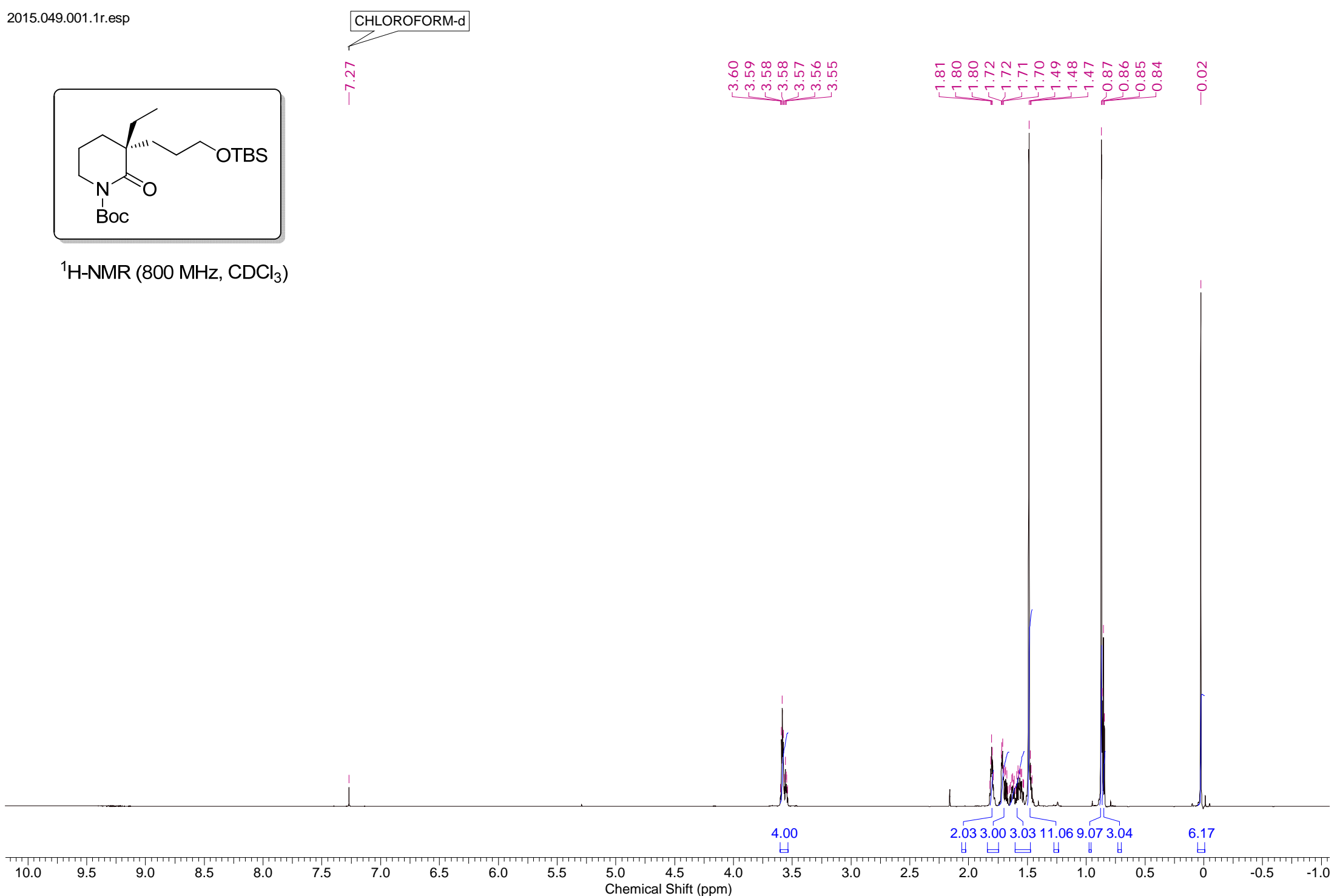


SKB BOC OTBS.004.001.1R.ESP

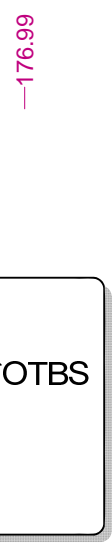

${ }^{13} \mathrm{C}-\mathrm{NMR}\left(101 \mathrm{MHz}, \mathrm{CDCl}_{3}\right.$ )

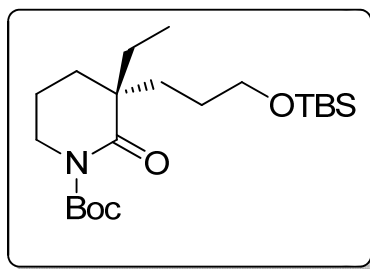

CHLOROFORM-d

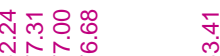

C

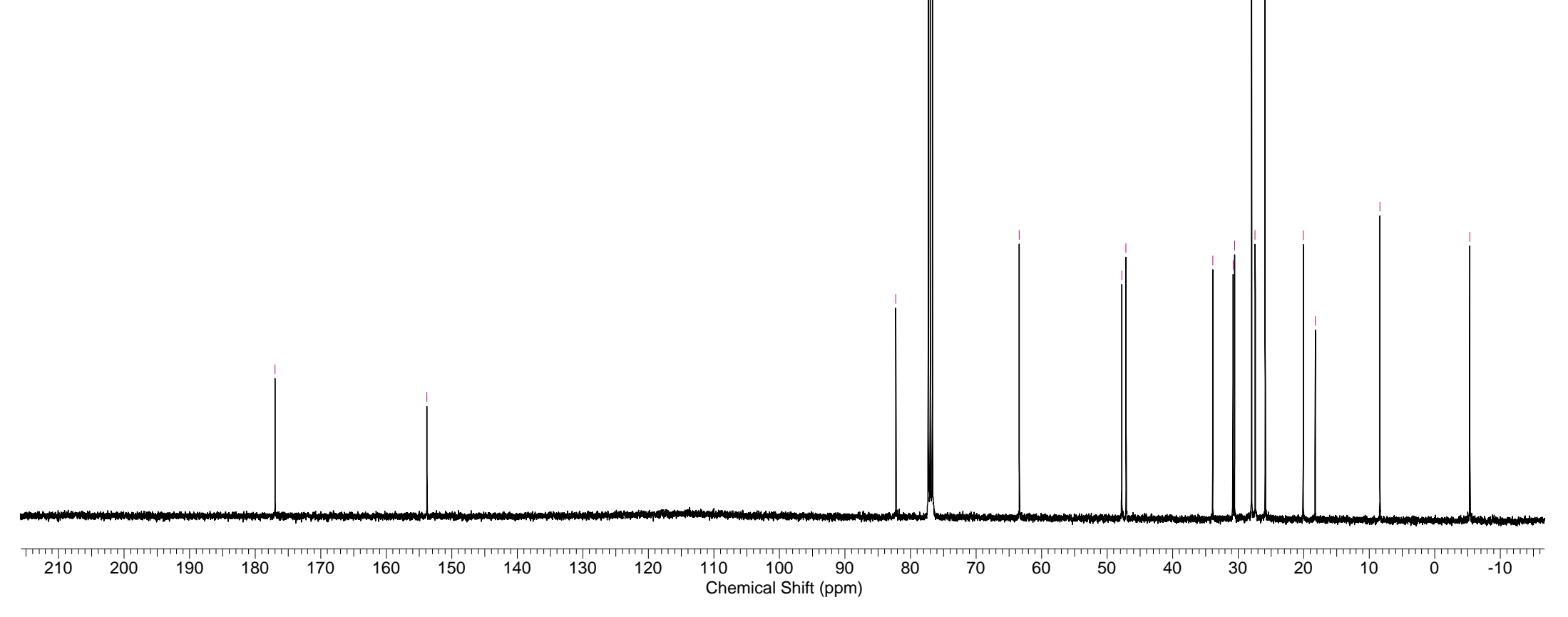


skb_boc_otbs1.002.001.1r.esp

CHLOROFORM-d

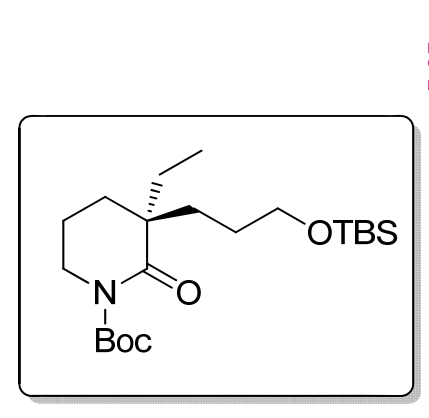

${ }^{1} \mathrm{H}-\mathrm{NMR}\left(400 \mathrm{MHz}, \mathrm{CDCl}_{3}\right.$ )

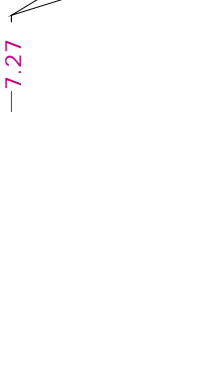


skb_boc_otbs1.003.001.1r.esp

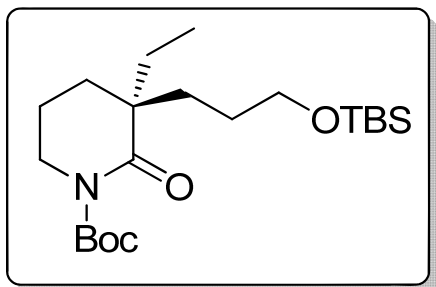

${ }^{13} \mathrm{C}-\mathrm{NMR}\left(101 \mathrm{MHz}, \mathrm{CDCl}_{3}\right)$

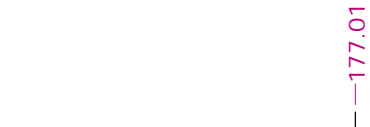

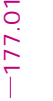

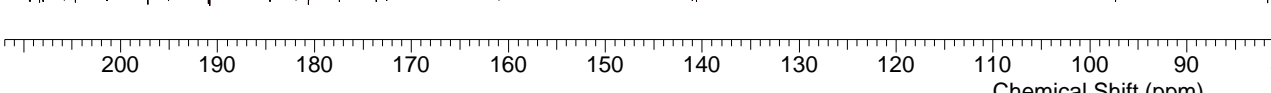

CHLOROFORM-d

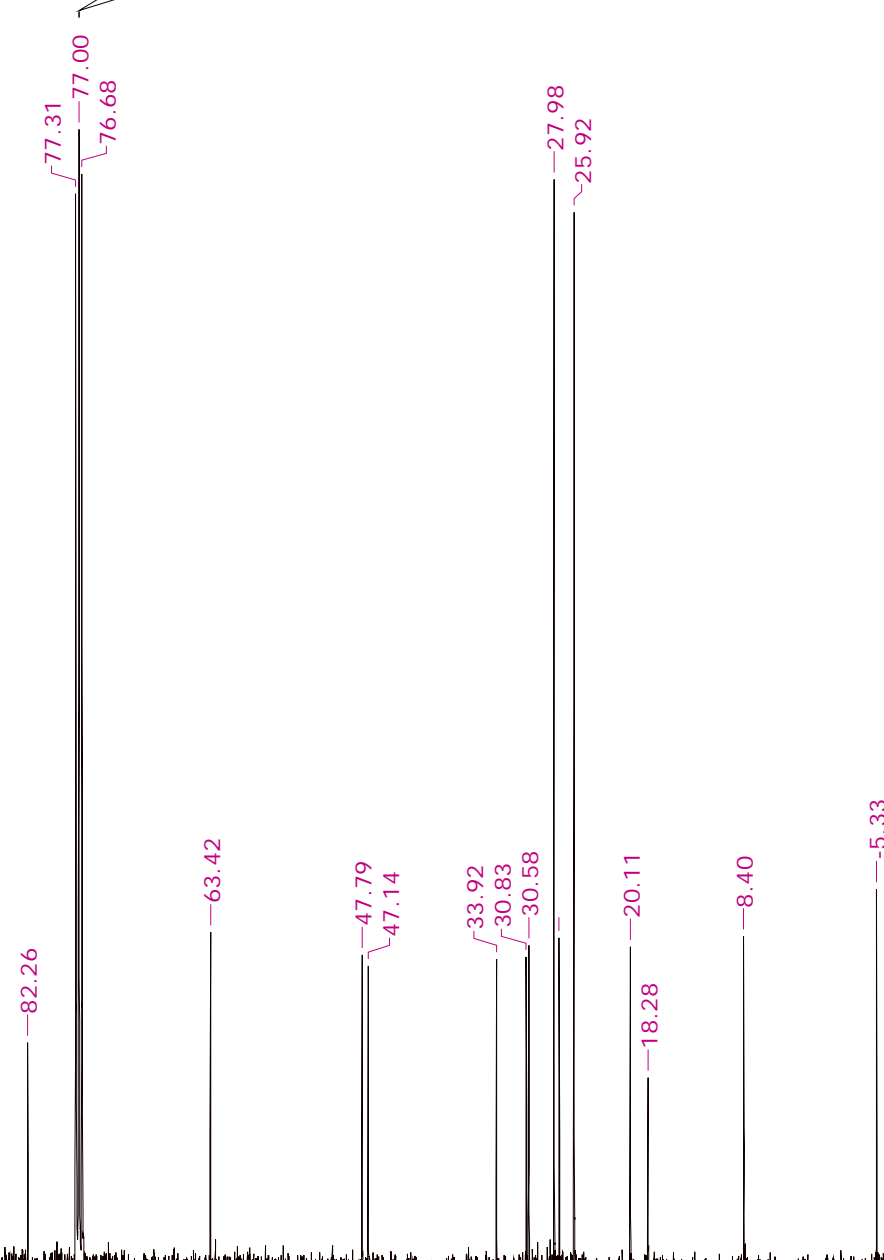

$\stackrel{m}{m}$ 


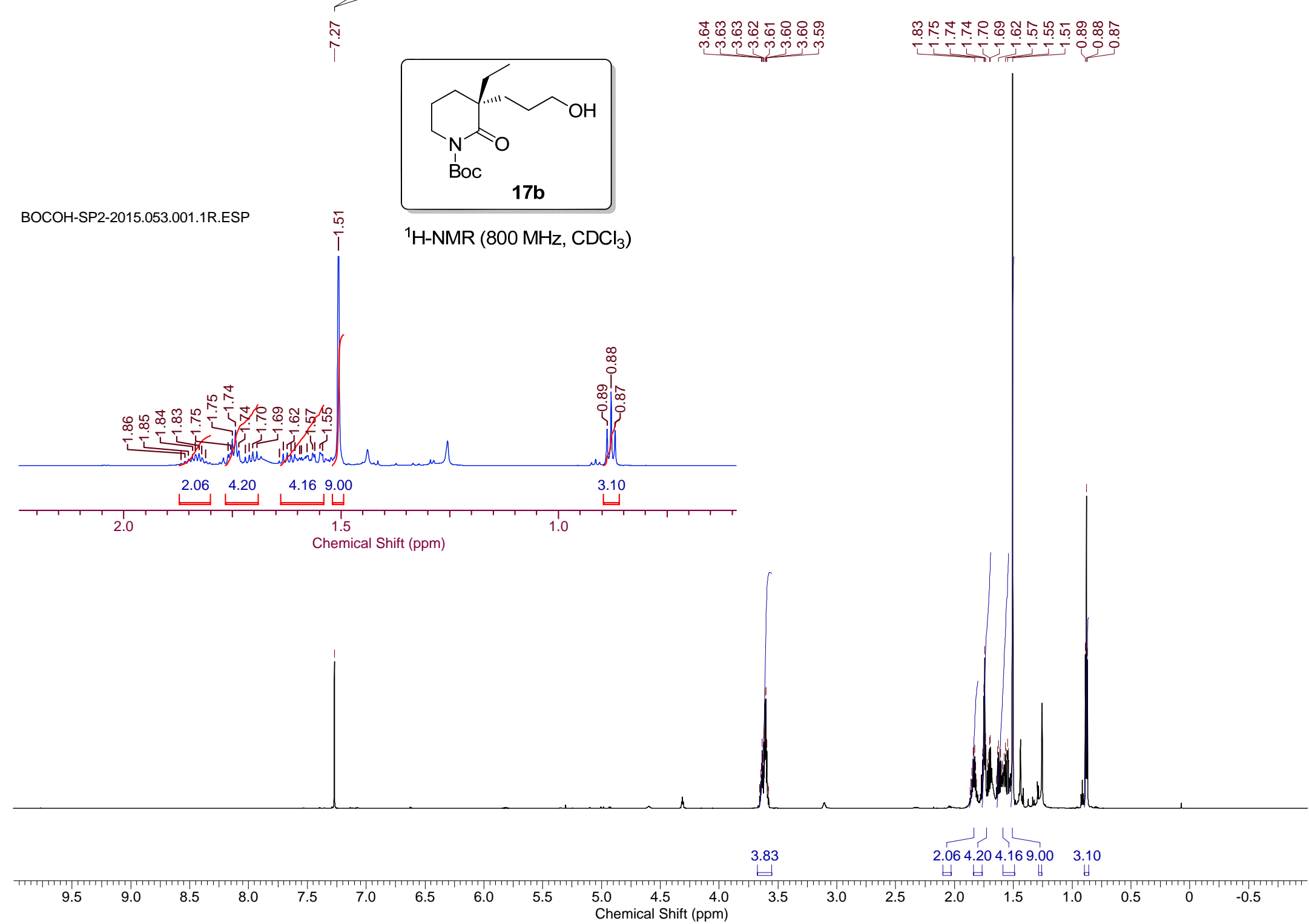


SKB BOCOH_PURE2.002.001.1R.ESP

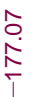

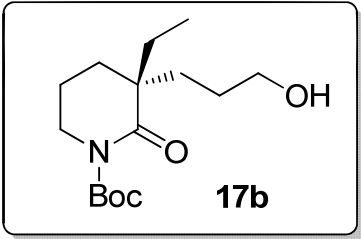

${ }^{13} \mathrm{C}-\mathrm{NMR}\left(101 \mathrm{MHz}, \mathrm{CDCl}_{3}\right)$
CHLOROFORM-d

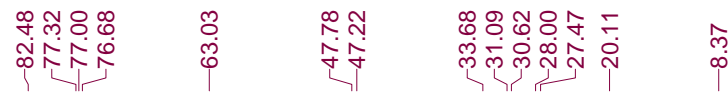

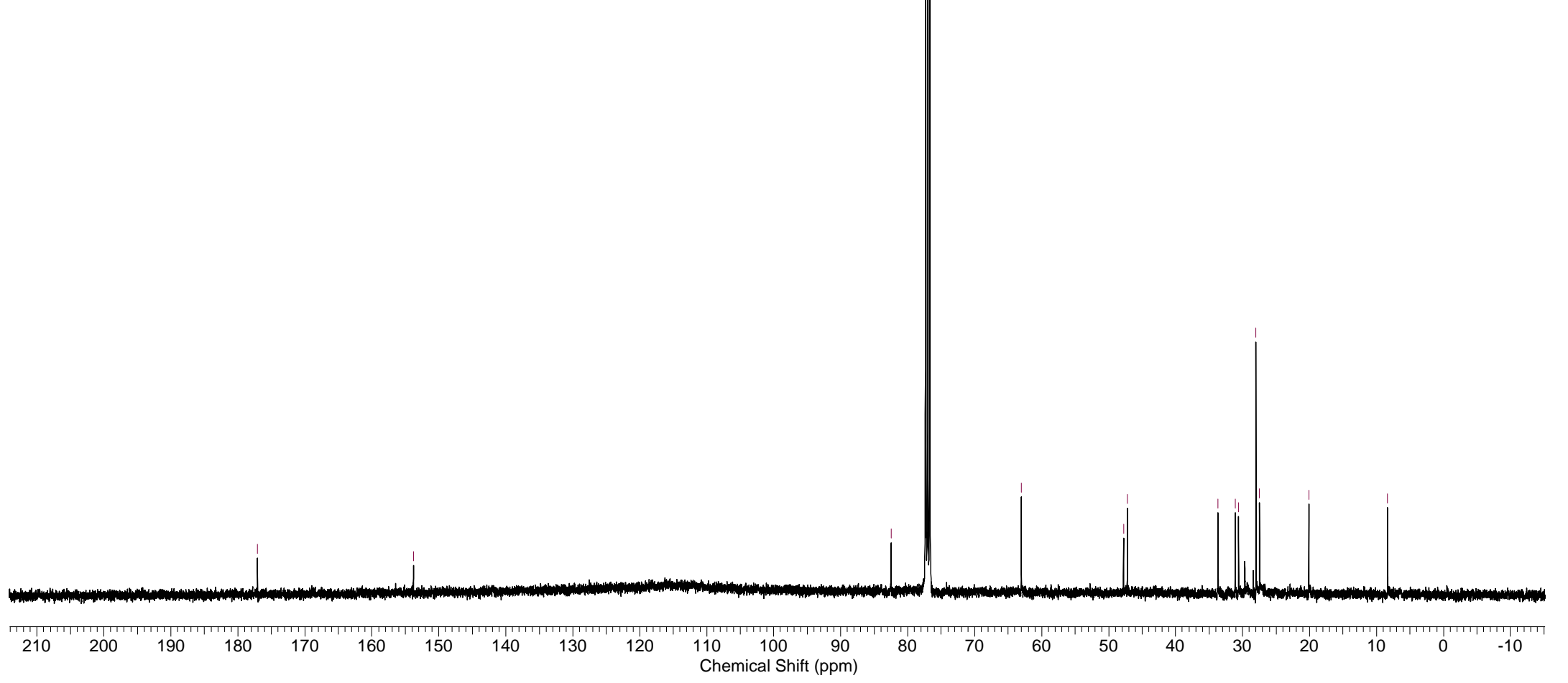




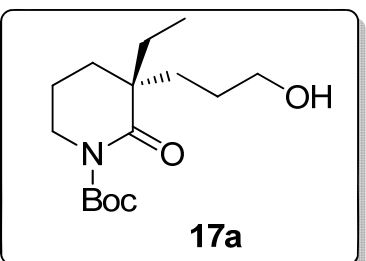

${ }^{1} \mathrm{H}-\mathrm{NMR}\left(400 \mathrm{MHz}, \mathrm{CDCl}_{3}\right.$ )

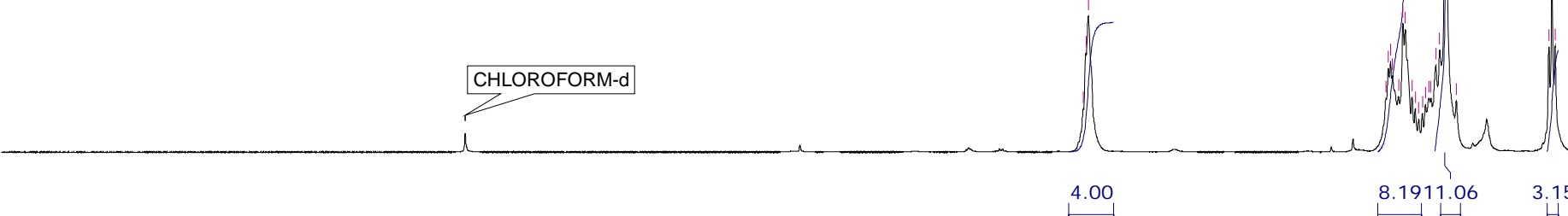

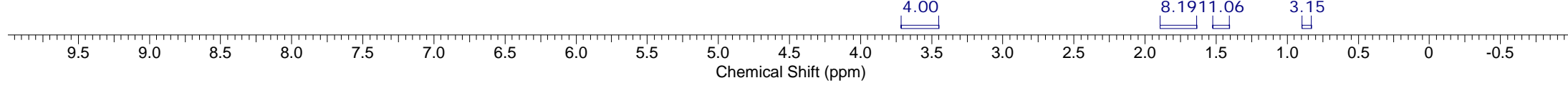




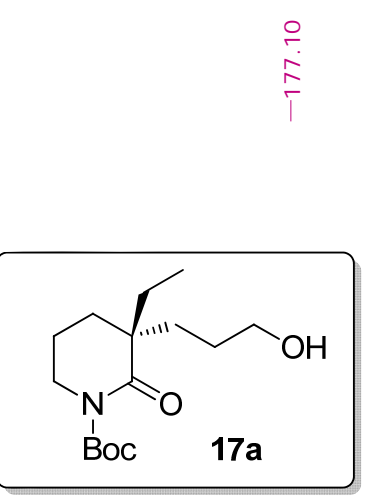

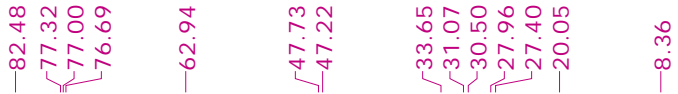

${ }^{13} \mathrm{C}-\mathrm{NMR}\left(101 \mathrm{MHz}, \mathrm{CDCl}_{3}\right)$

Diblow

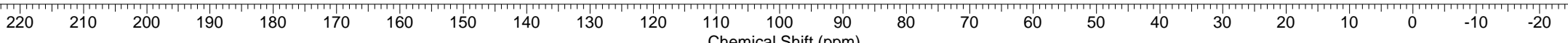
Chemical Shift (ppm) 
1H_BOCALD2015.005.001.1R.ESP

$\stackrel{\hat{n}}{i}$

${ }^{1} \mathrm{H}-\mathrm{NMR}\left(800 \mathrm{MHz}, \mathrm{CDCl}_{3}\right)$

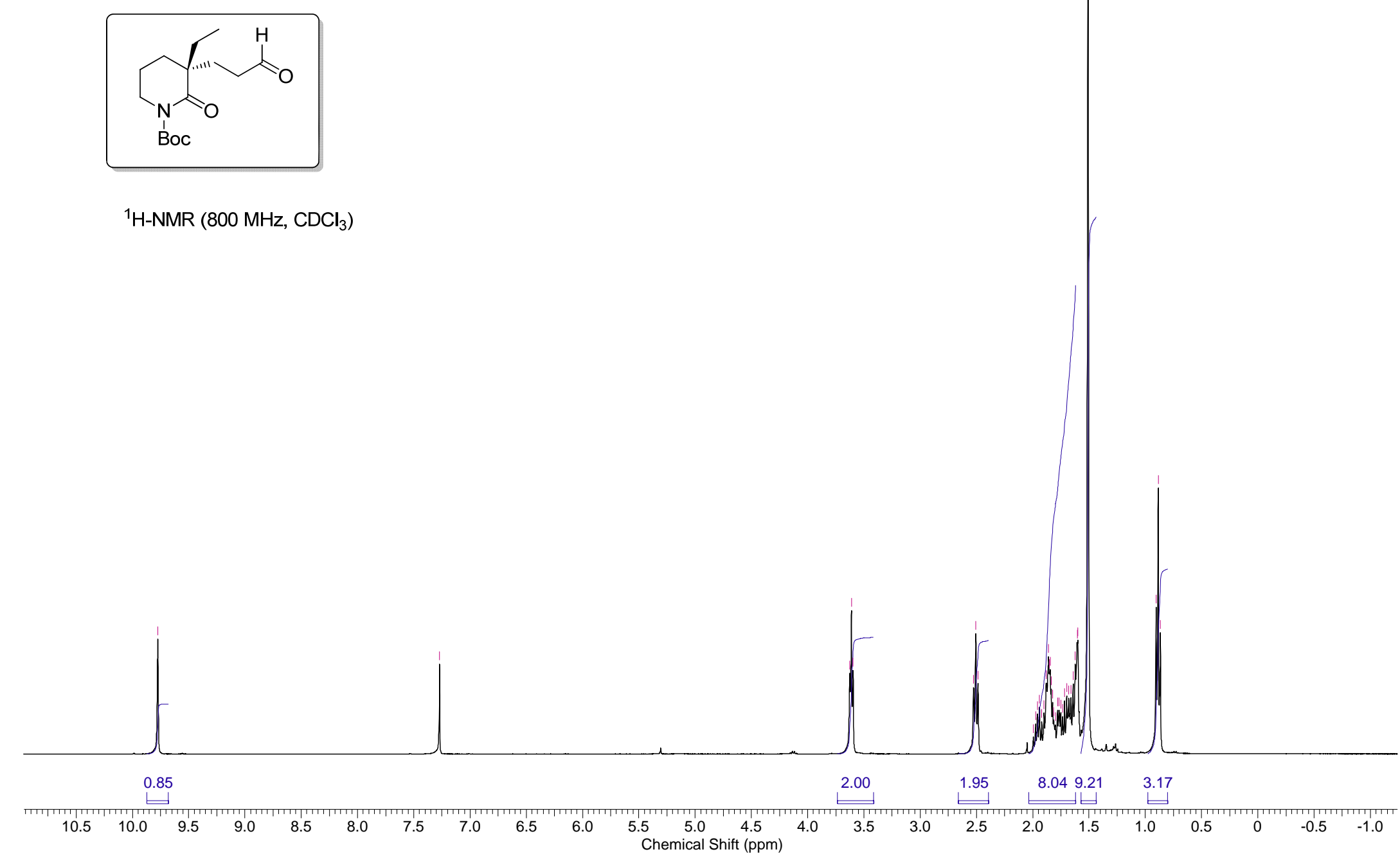

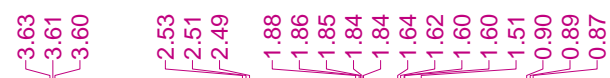

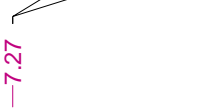




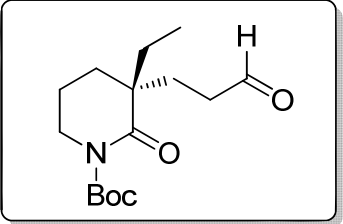

${ }^{13} \mathrm{C}-\mathrm{NMR}\left(201 \mathrm{MHz}, \mathrm{CDCl}_{3}\right.$ )

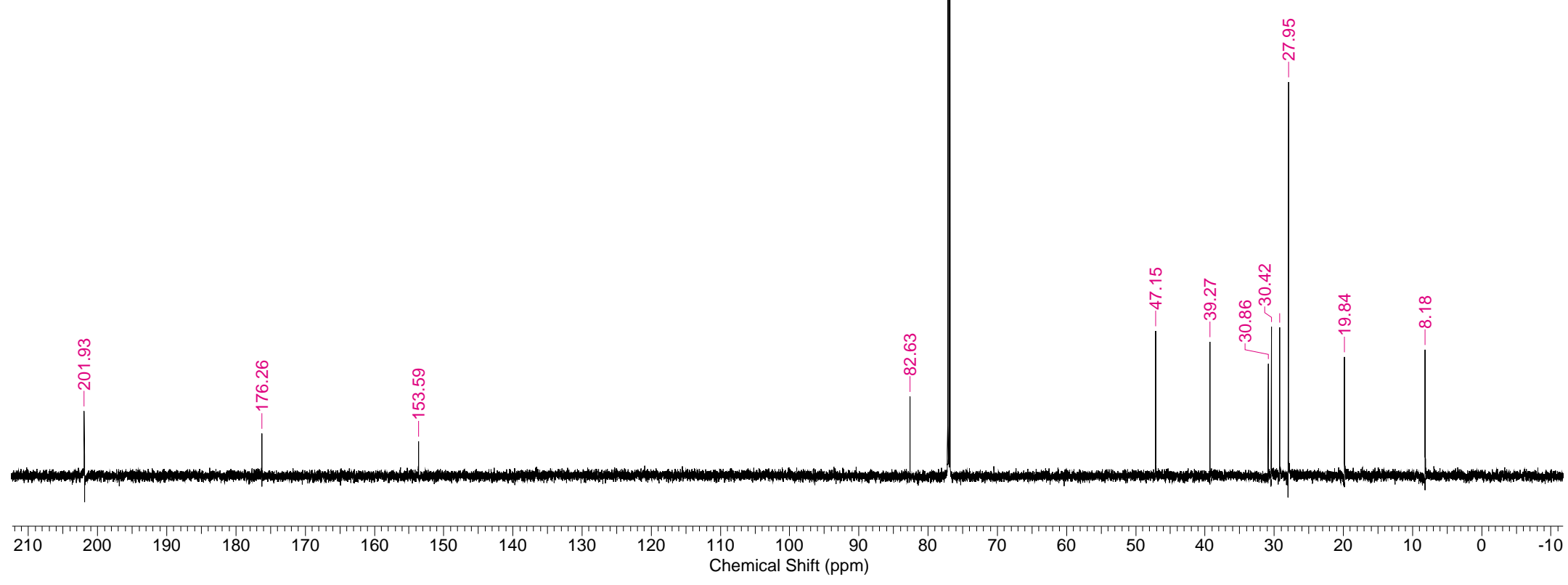


skb_boc_ald_1.002.001.1r.esp

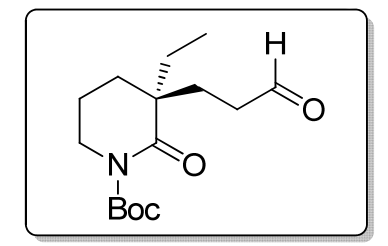

${ }^{1} \mathrm{H}-\mathrm{NMR}(400 \mathrm{MHz}, \mathrm{CDCl} 3$ )

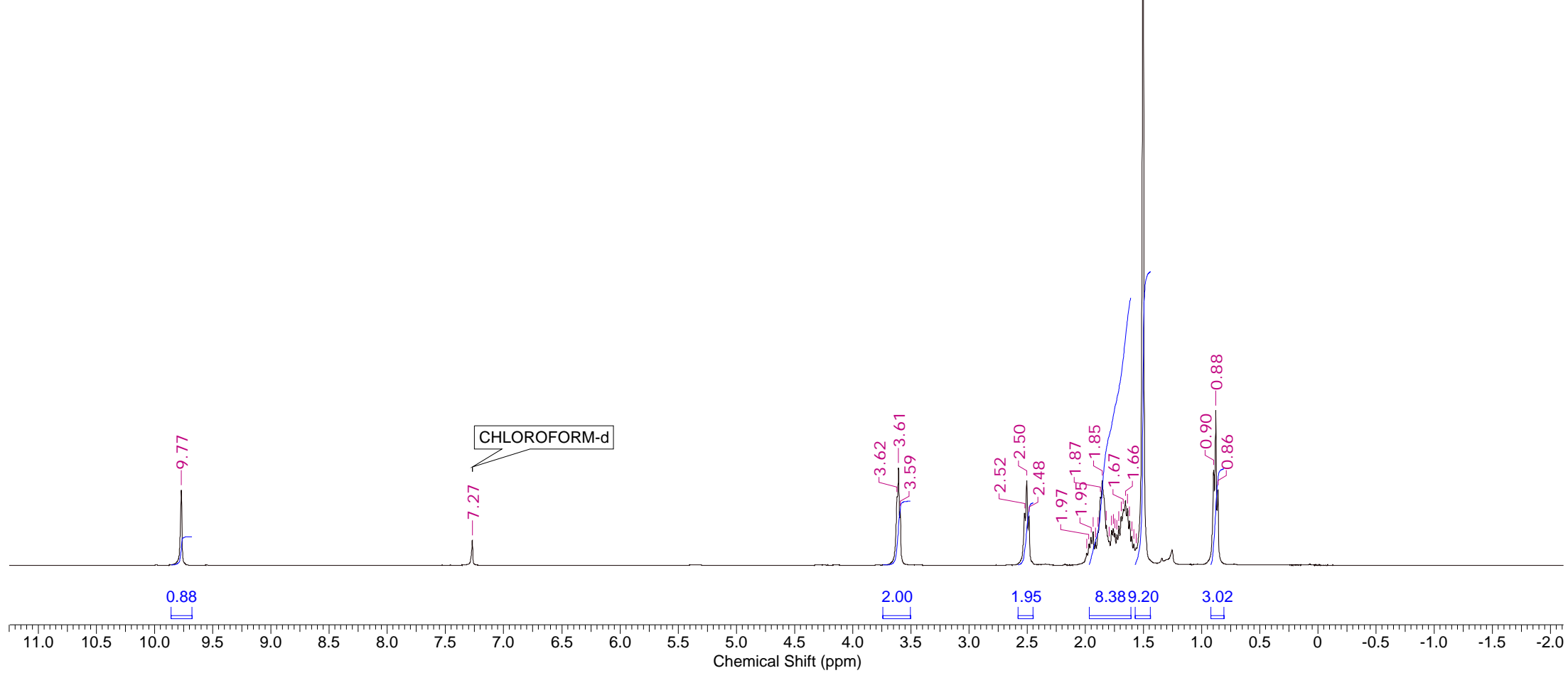


SKB BOC ALD 1.003.001.1R.ESP

$$
\text { के }
$$

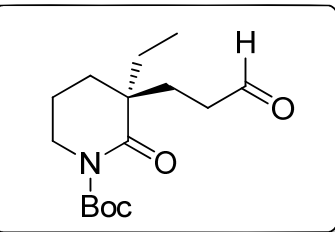

${ }^{13} \mathrm{C}$ NMR (101 MHz, $\mathrm{CDCl}_{3}$ )
CHLOROFORM-d

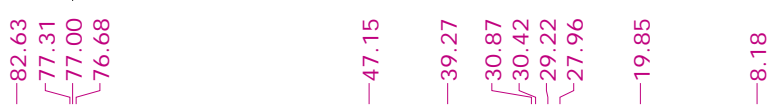




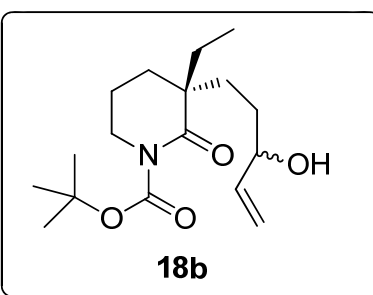

$18 b$

${ }^{1} \mathrm{H}-\mathrm{NMR}\left(400 \mathrm{MHz}, \mathrm{CDCl}_{3}\right)$

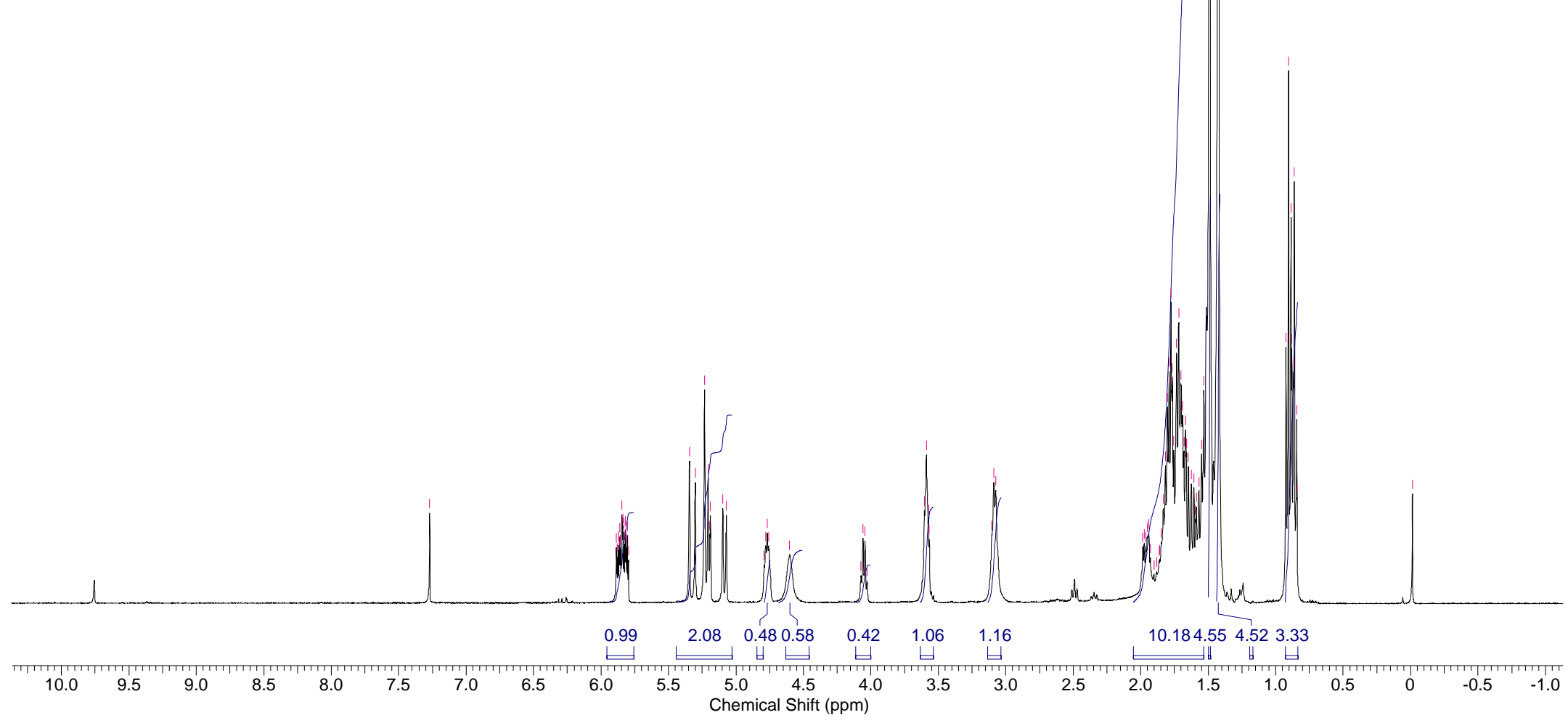




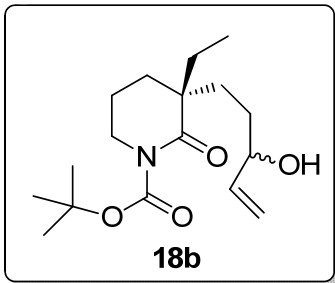

${ }^{13} \mathrm{C}-\mathrm{NMR}\left(101 \mathrm{MHz}, \mathrm{CDCl}_{3}\right.$ )

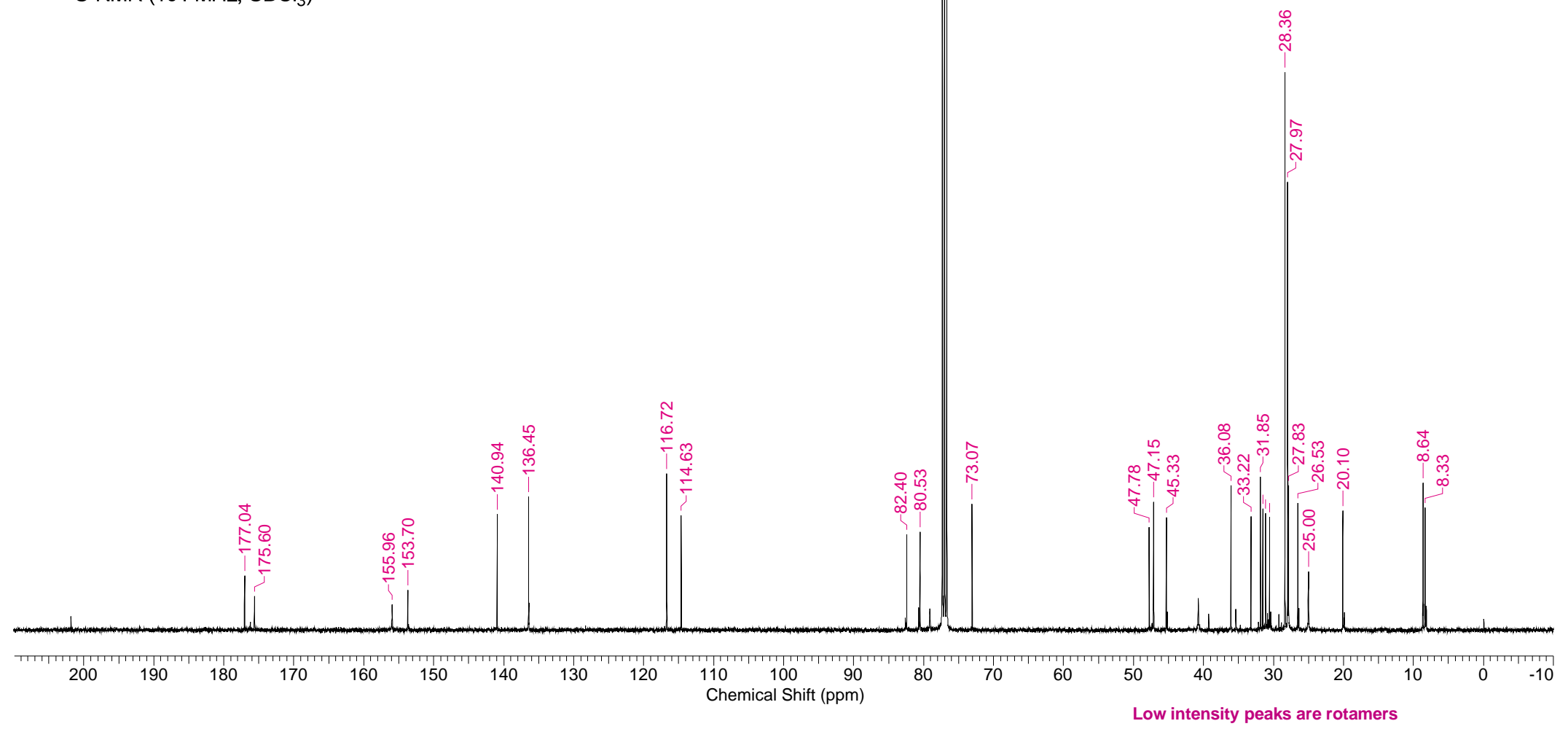




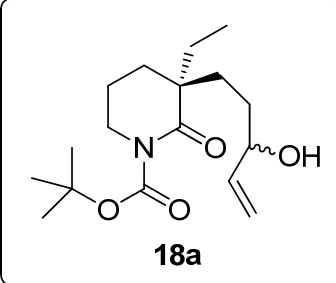

${ }^{1} \mathrm{H}-\mathrm{NMR}\left(800 \mathrm{MHz}, \mathrm{CDCl}_{3}\right)$

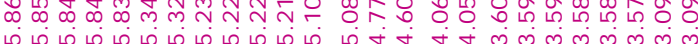

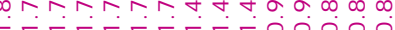

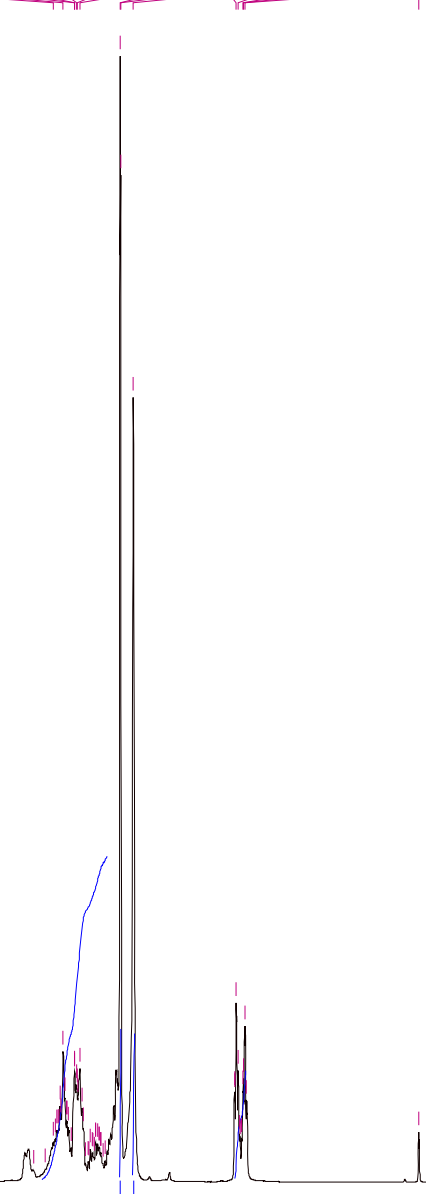

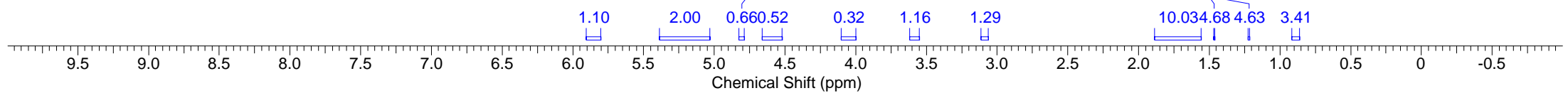




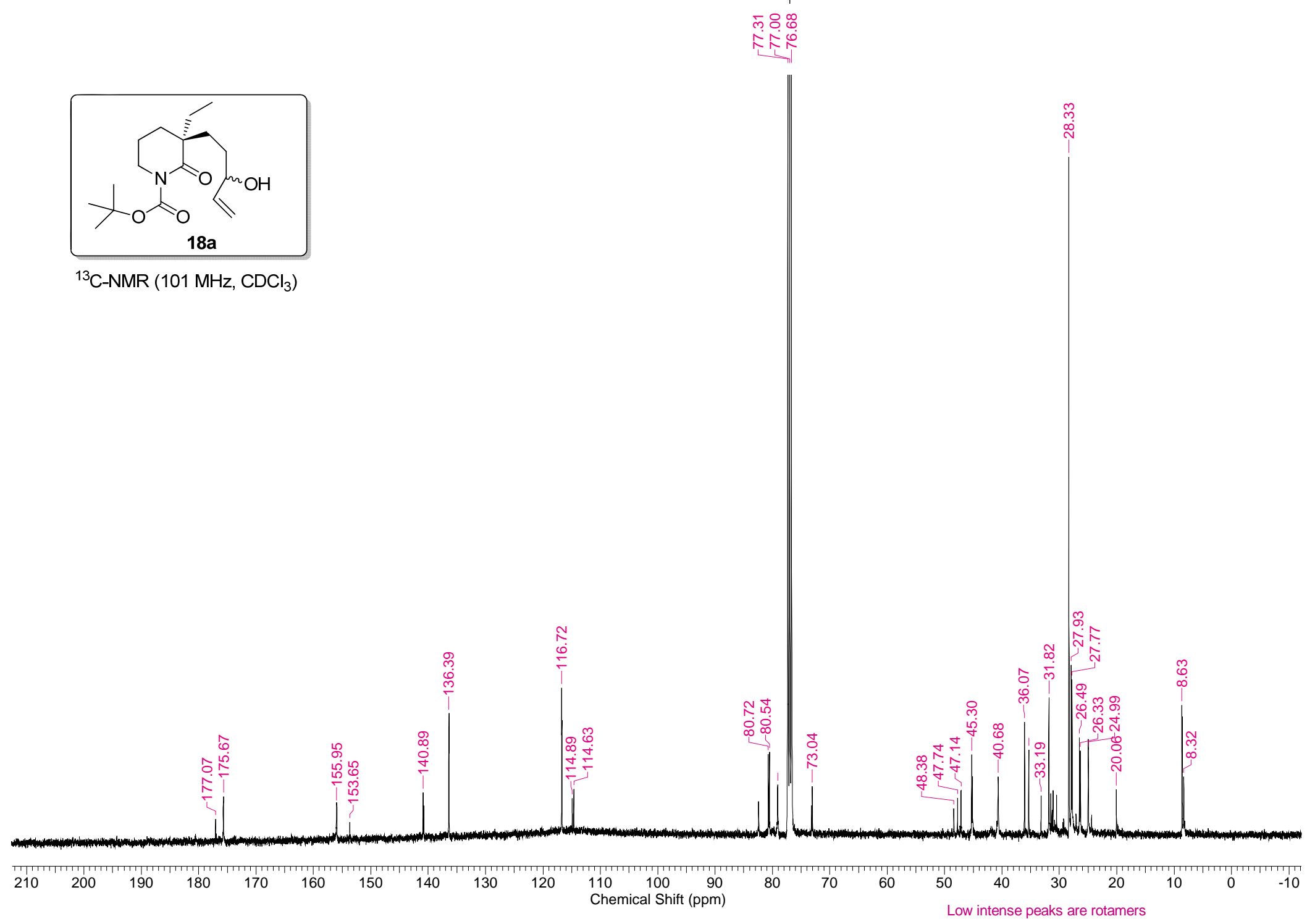




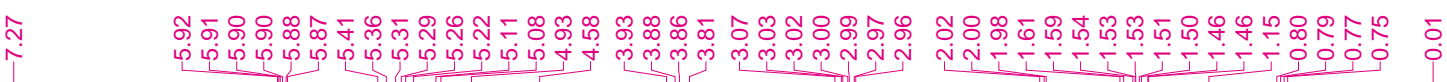

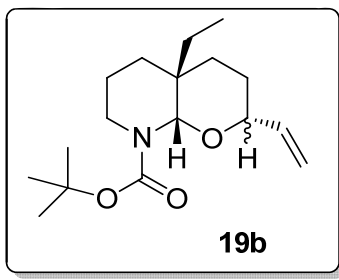

${ }^{1} \mathrm{H}-\mathrm{NMR}\left(400 \mathrm{MHz}, \mathrm{CDCl}_{3}\right)$

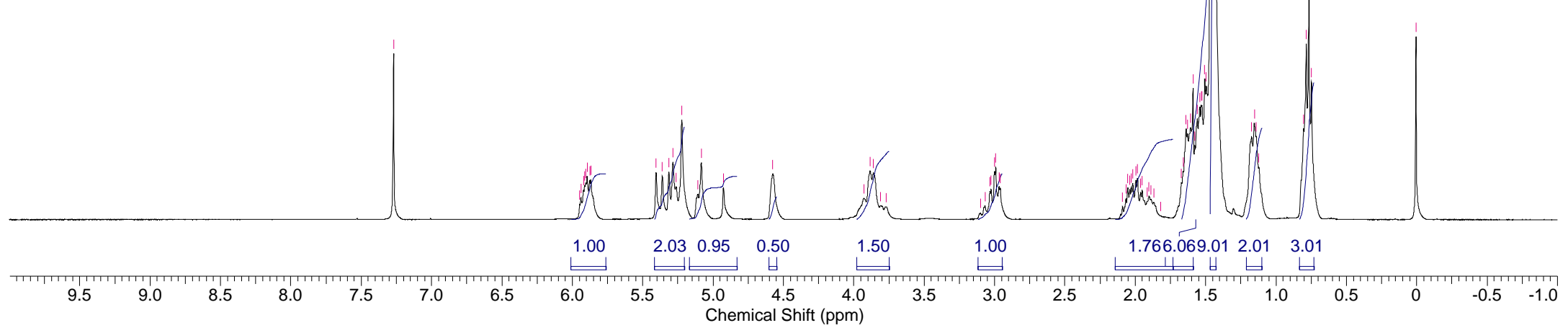



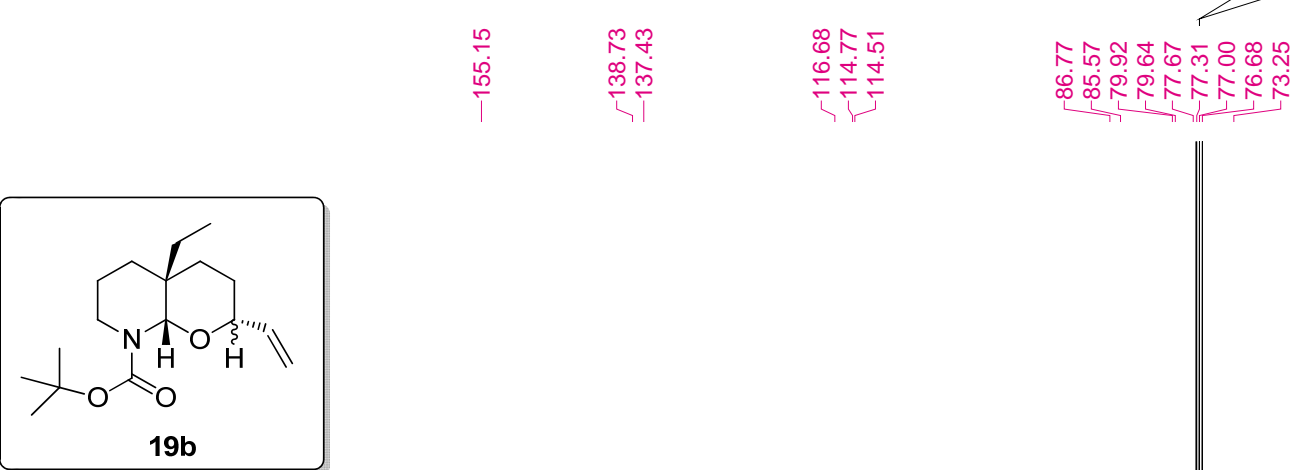

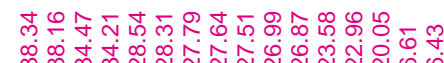

${ }^{13} \mathrm{C}-\mathrm{NMR}\left(101 \mathrm{MHz}, \mathrm{CDCl}_{3}\right.$ )

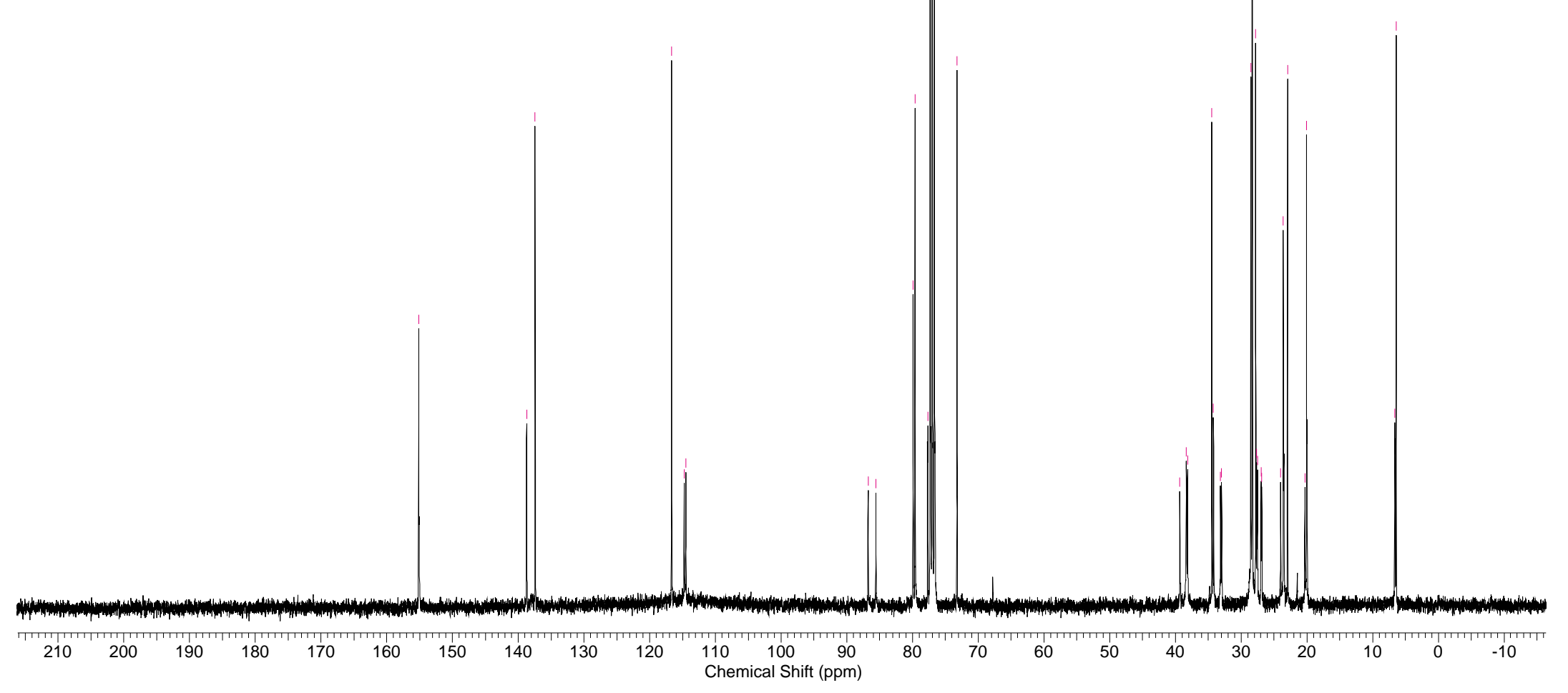




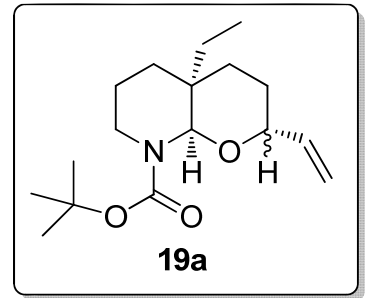

${ }^{1} \mathrm{H}-\mathrm{NMR}\left(400 \mathrm{MHz}, \mathrm{CDCl}_{3}\right.$ )

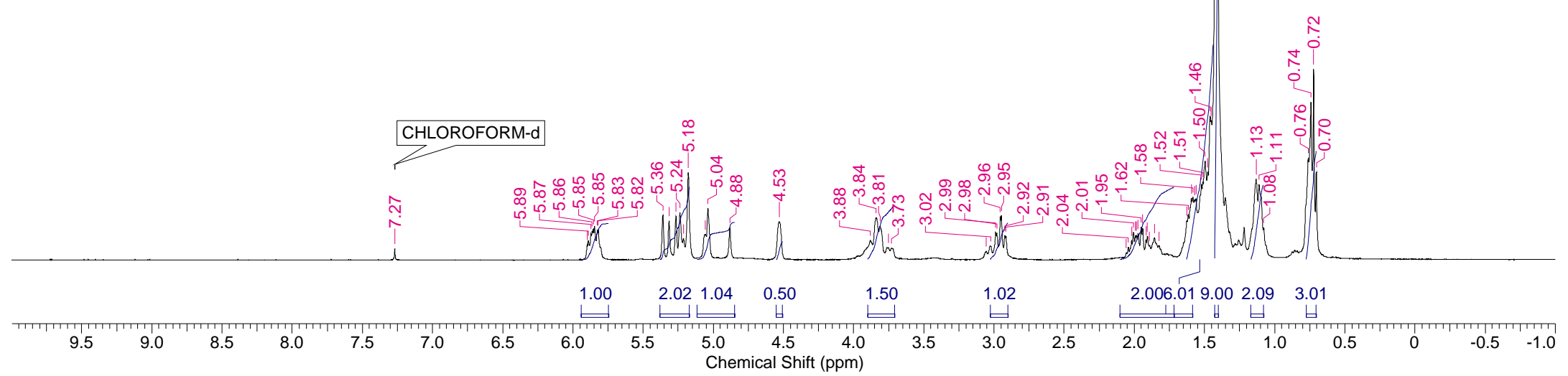


CHLOROFORM-d

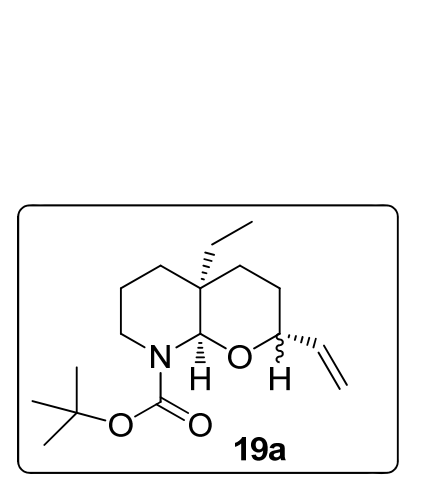

\begin{tabular}{|c|c|c|}
\hline 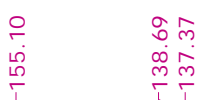 & 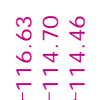 & 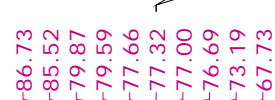 \\
\hline
\end{tabular}

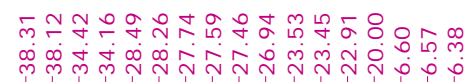

${ }^{13} \mathrm{C} \mathrm{NMR}\left(\mathrm{CDCl}_{3}, 101 \mathrm{MHz}\right)$

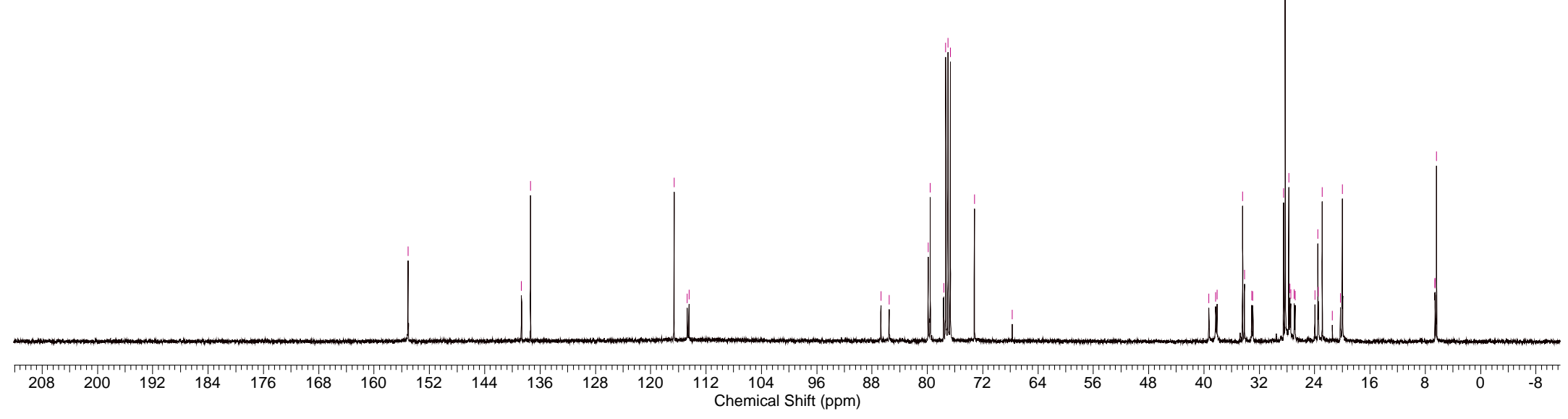




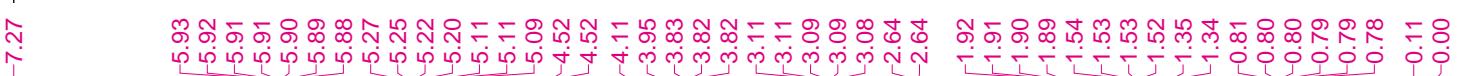

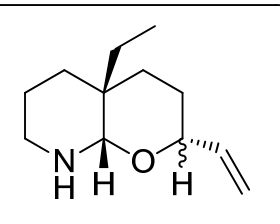

1H-NMR (800MHz, CDCl3)

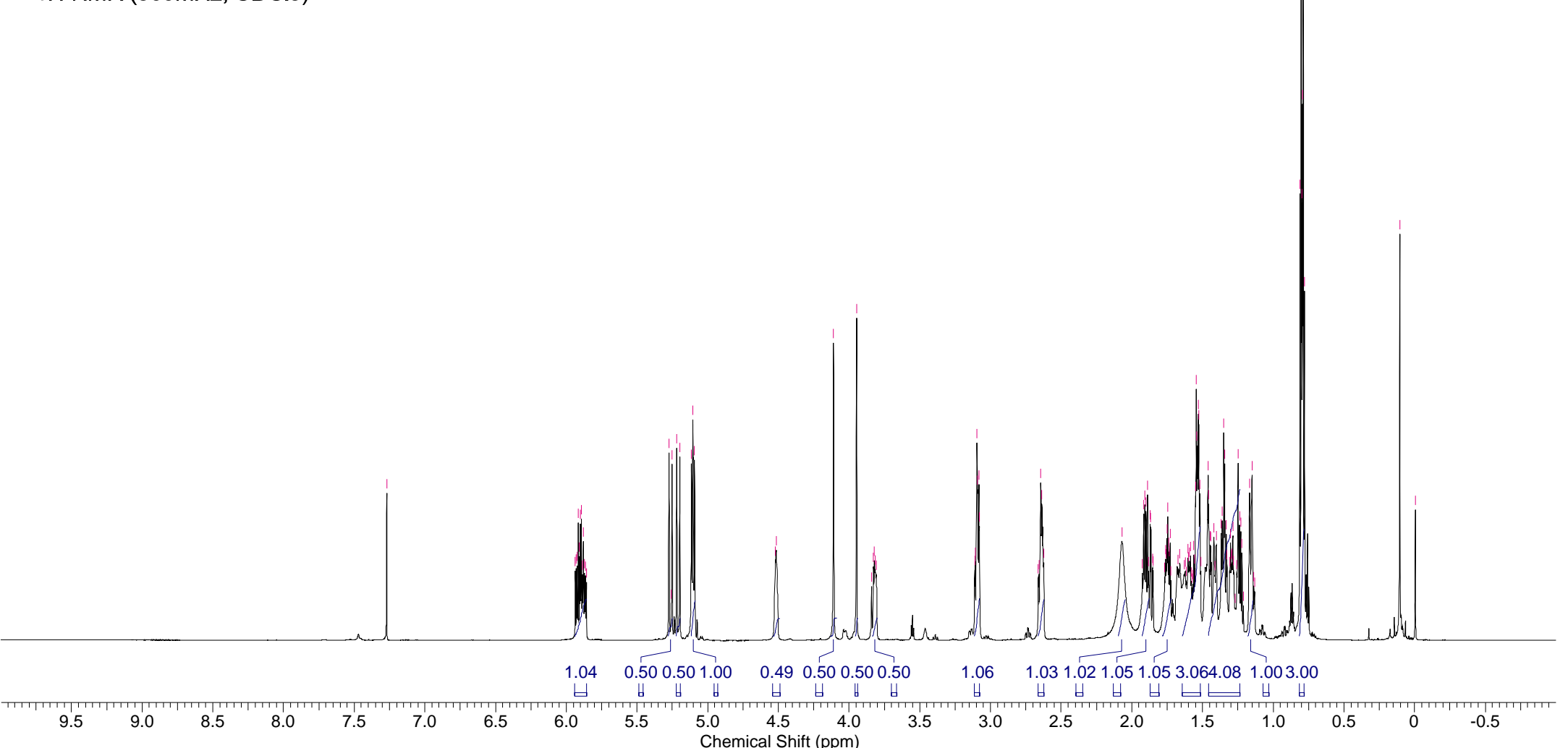




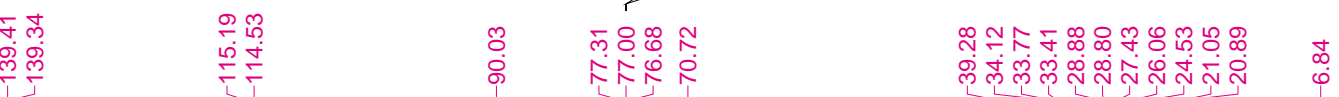

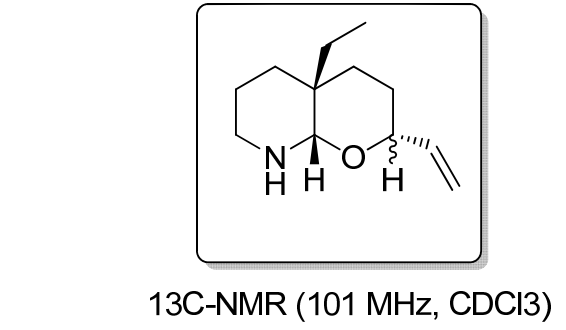

13C-NMR (101 MHz, CDCl3)

更

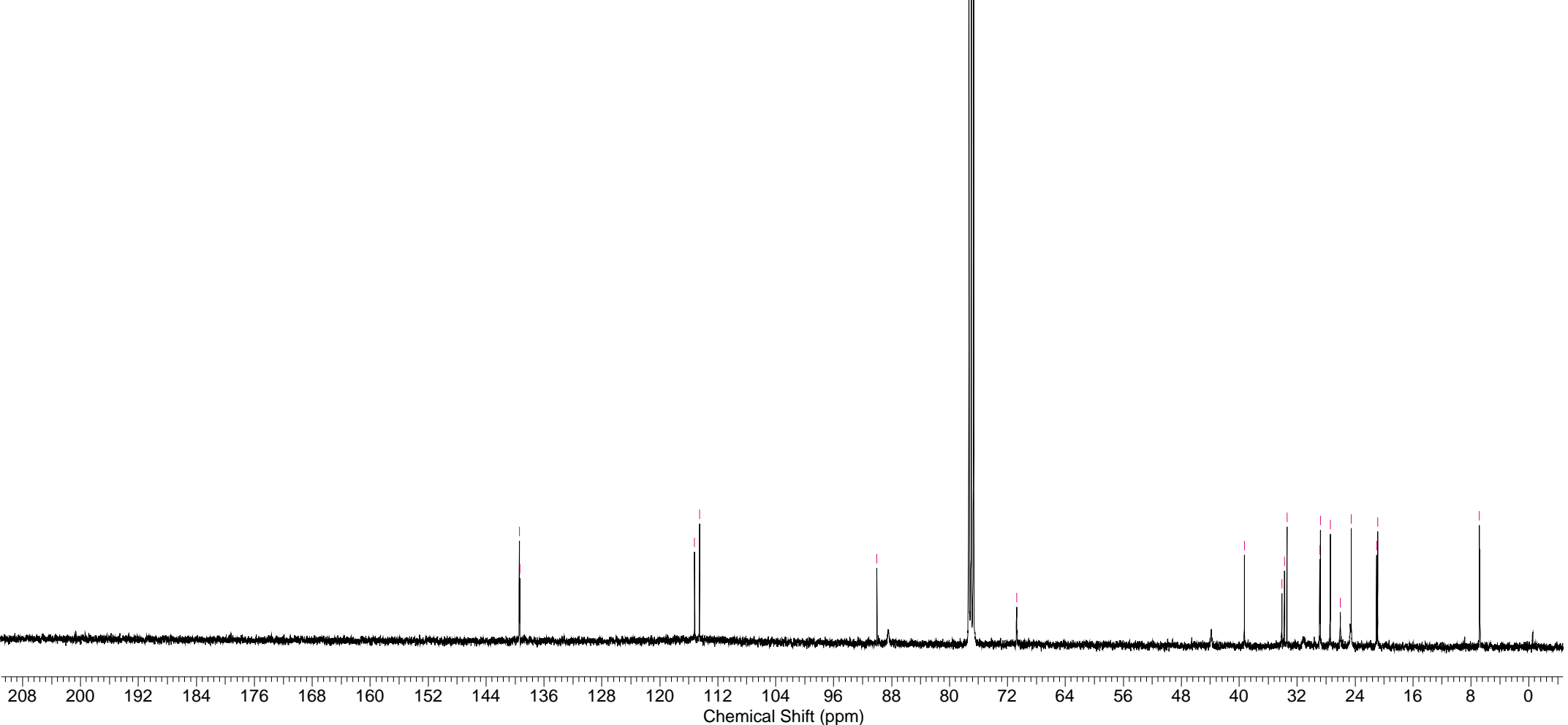




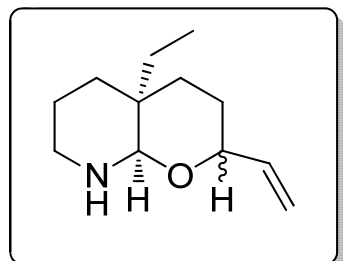

1H-NMR (400MHz, CDCl3)

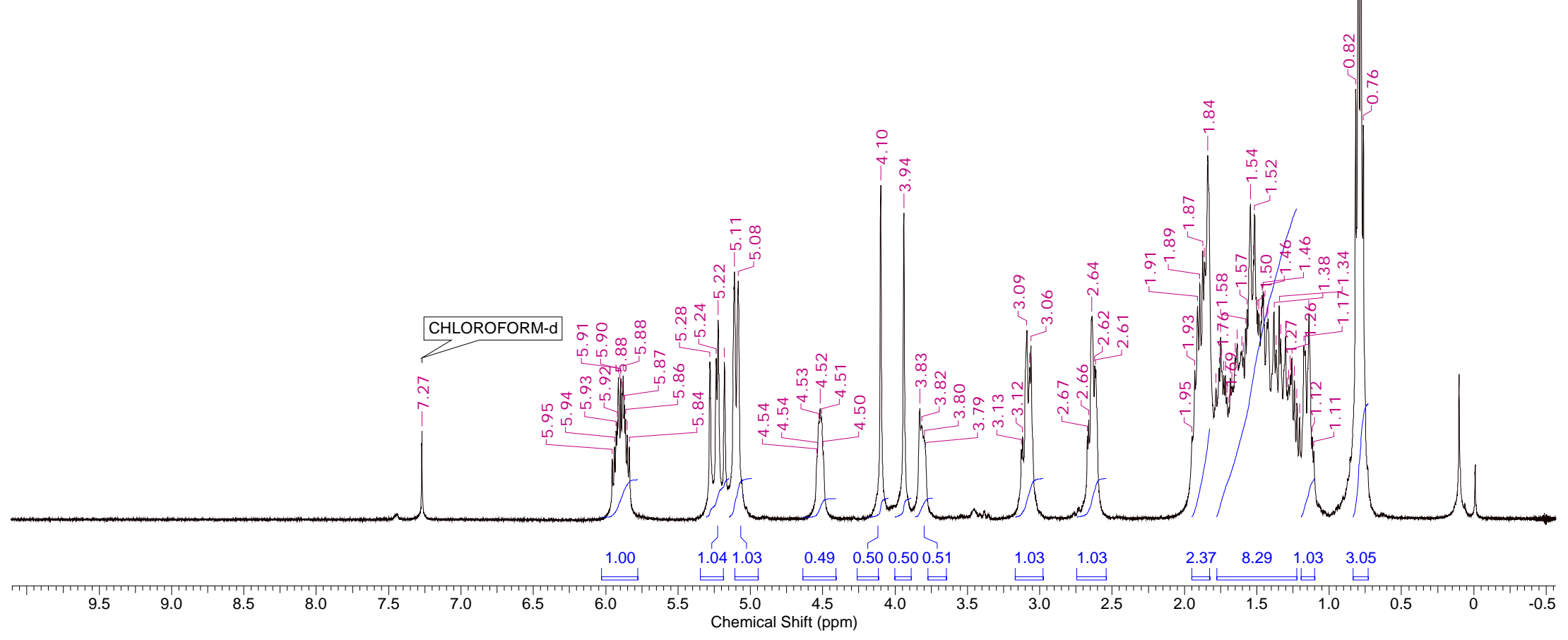


SKB BCYAM_A.007.001.1R.ESP

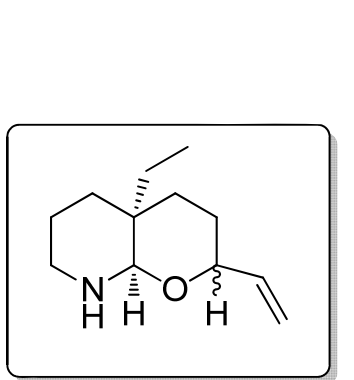

13C-NMR (101 MHz, CDCI3)
CHLOROFORM-d

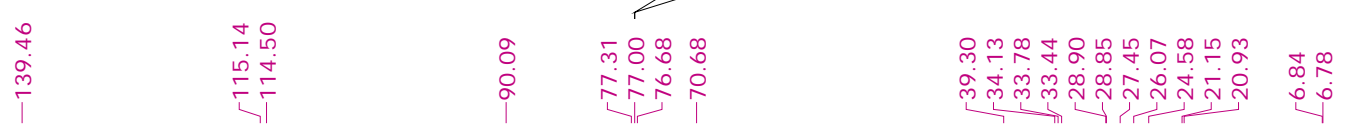

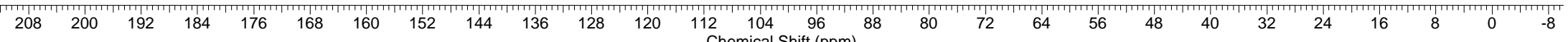




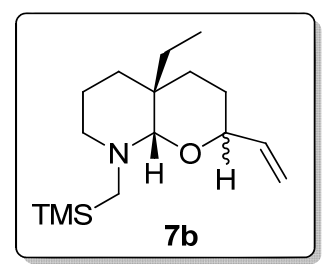

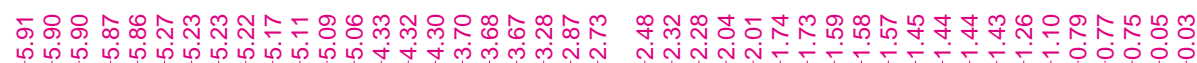

1H-NMR (40OMHz, CDCl3)

1H-skb_casm.001.001.1r.esp

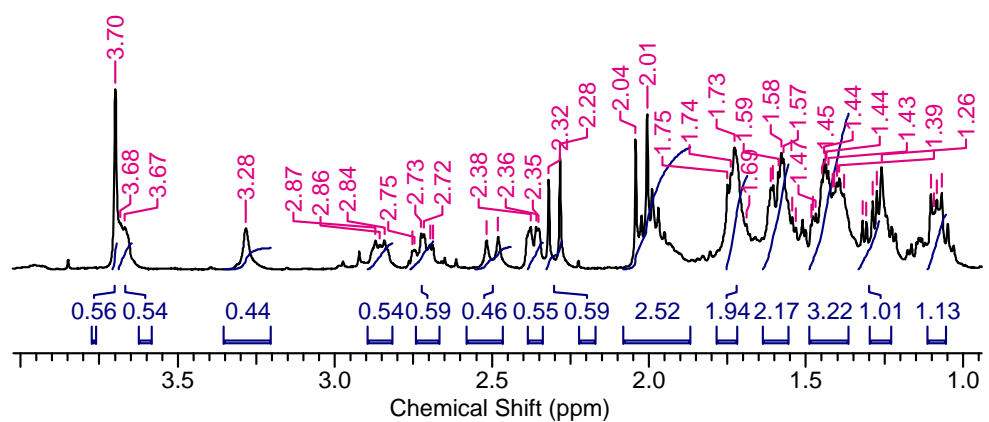

$1.06 \quad 1.181 .05 \quad 0.450 .560 .540 .440 .540 .590 .460 .550 .592 .521 .942 .173 .221 .011 .133 .259 .16$

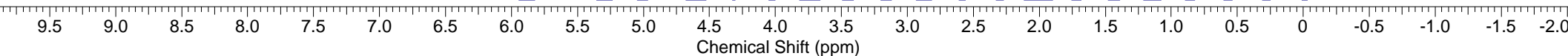




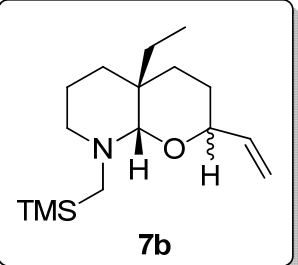

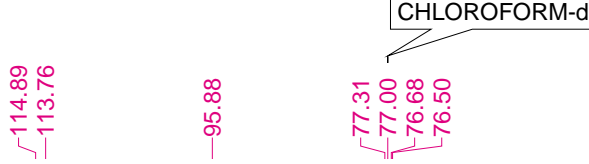

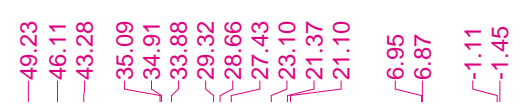

${ }^{13} \mathrm{C}-\mathrm{NMR}\left(101 \mathrm{MHz}, \mathrm{CDCl}_{3}\right)$

\section{1.}

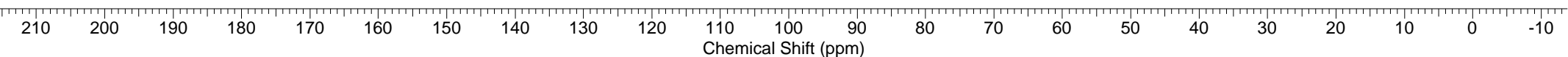




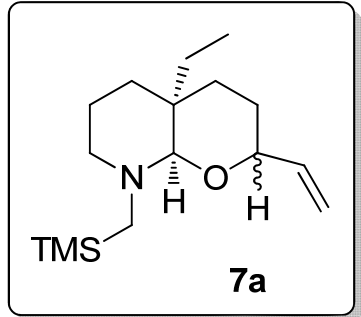

${ }^{1} \mathrm{H}-\mathrm{NMR}\left(400 \mathrm{MHz}, \mathrm{CDCl}_{3}\right.$ )

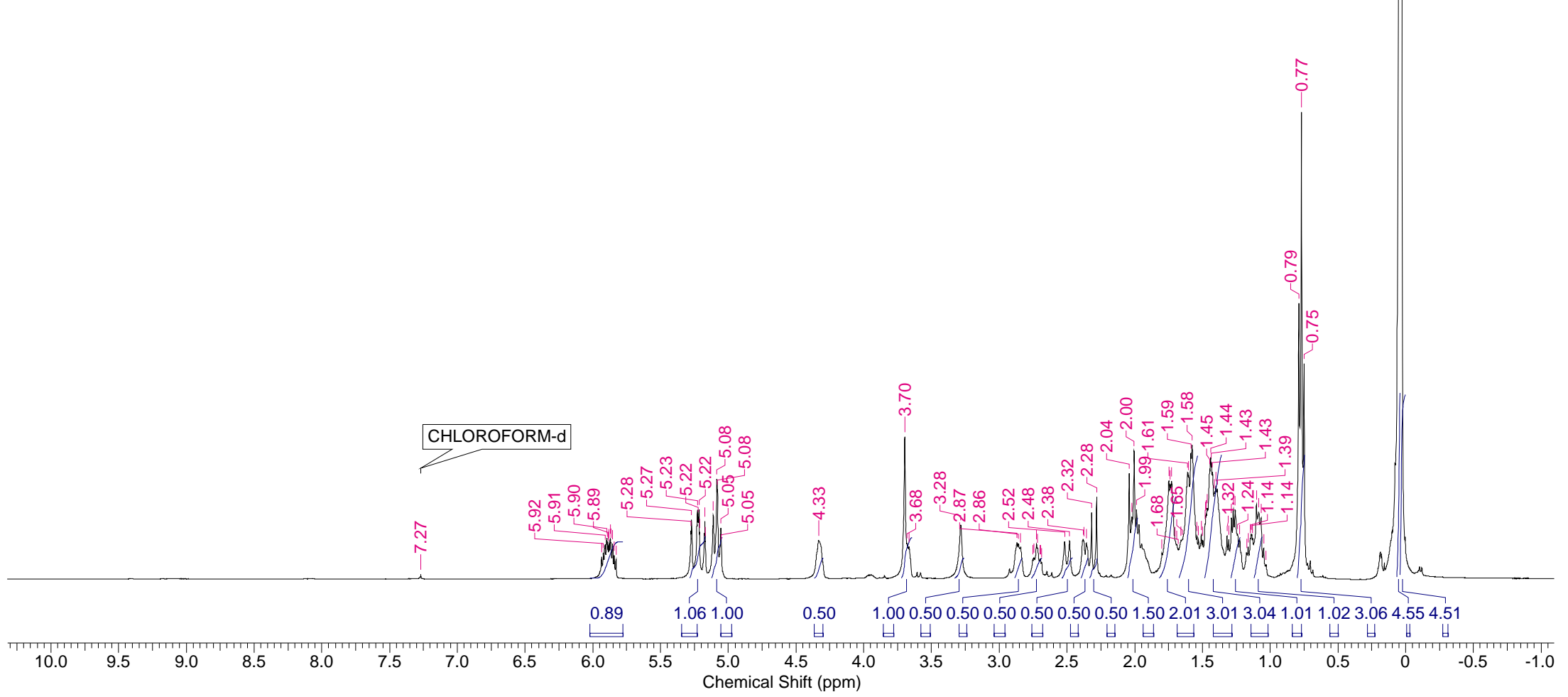




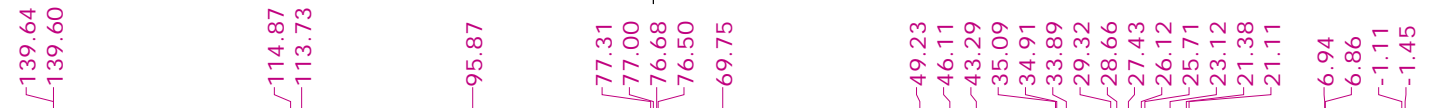

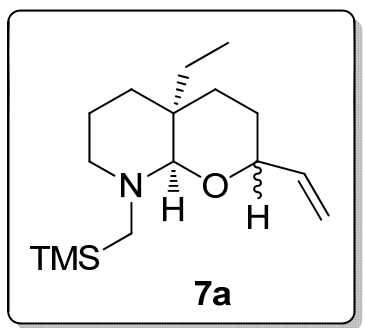

13C-NMR (101 MHz, CDCl3)

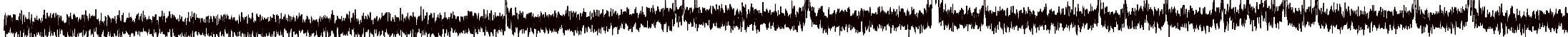

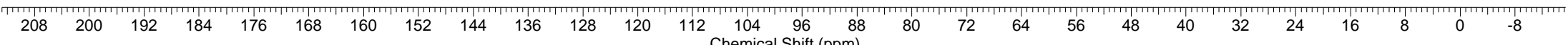




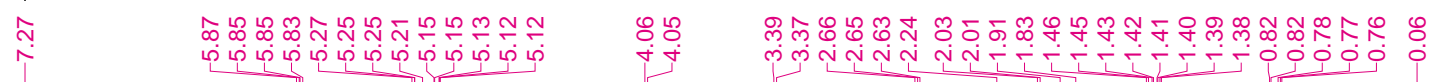

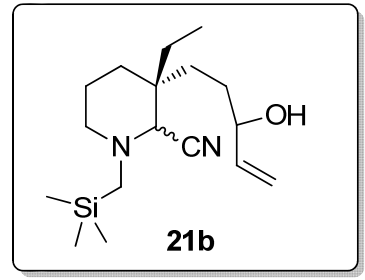

${ }^{1} \mathrm{H}-\mathrm{NMR}\left(400 \mathrm{MHz}, \mathrm{CDCl}_{3}\right)$ 
skb_CNOH 2.003.001.1r.esp

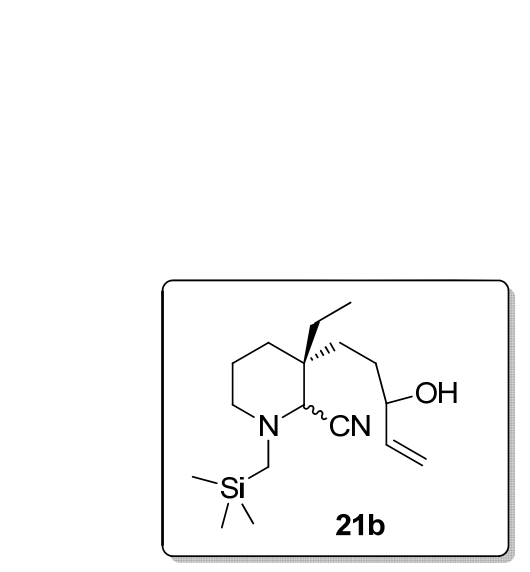

${ }^{13} \mathrm{C}-\mathrm{NMR}\left(101 \mathrm{MHz}, \mathrm{CDCl}_{3}\right)$
CHLOROFORM-d

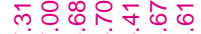

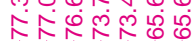

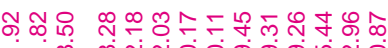 \\ 舟}

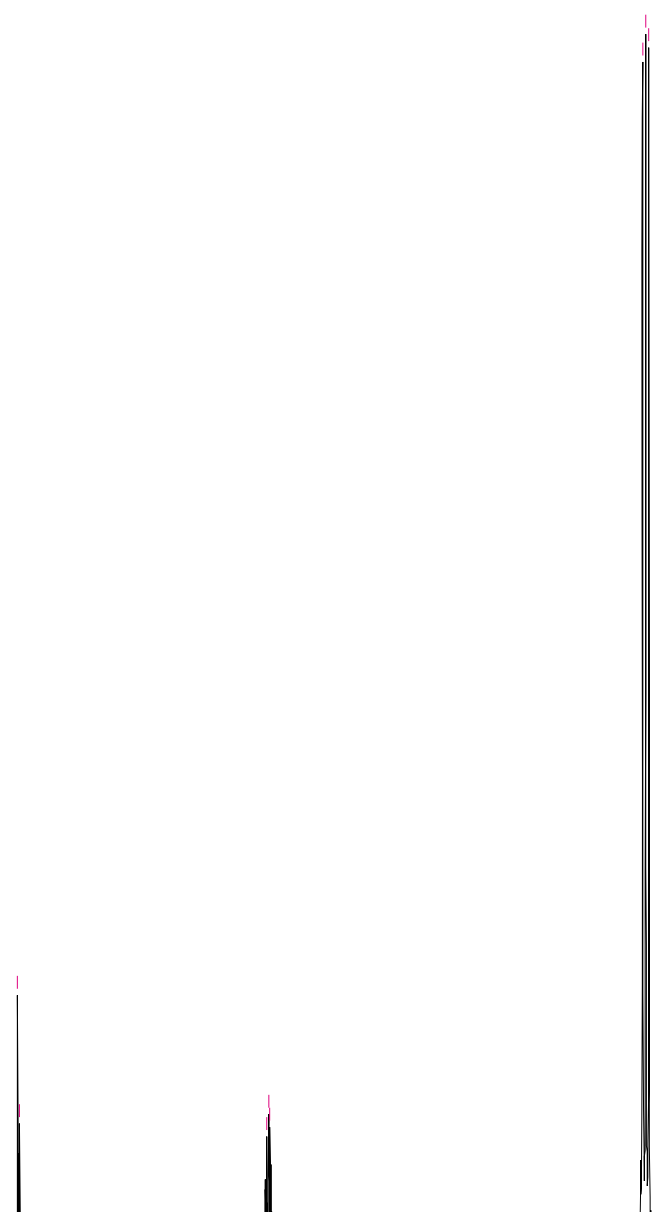

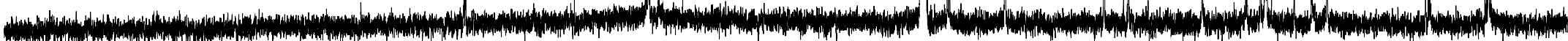

$\begin{array}{llllllllllllllllllllll}200 & 190 & 180 & 170 & 160 & 150 & 140 & 130 & 120 & 110 & 100 & 90 & 80 & 70 & 60 & 50 & 40 & 30 & 20 & 10 & 0 & -10\end{array}$




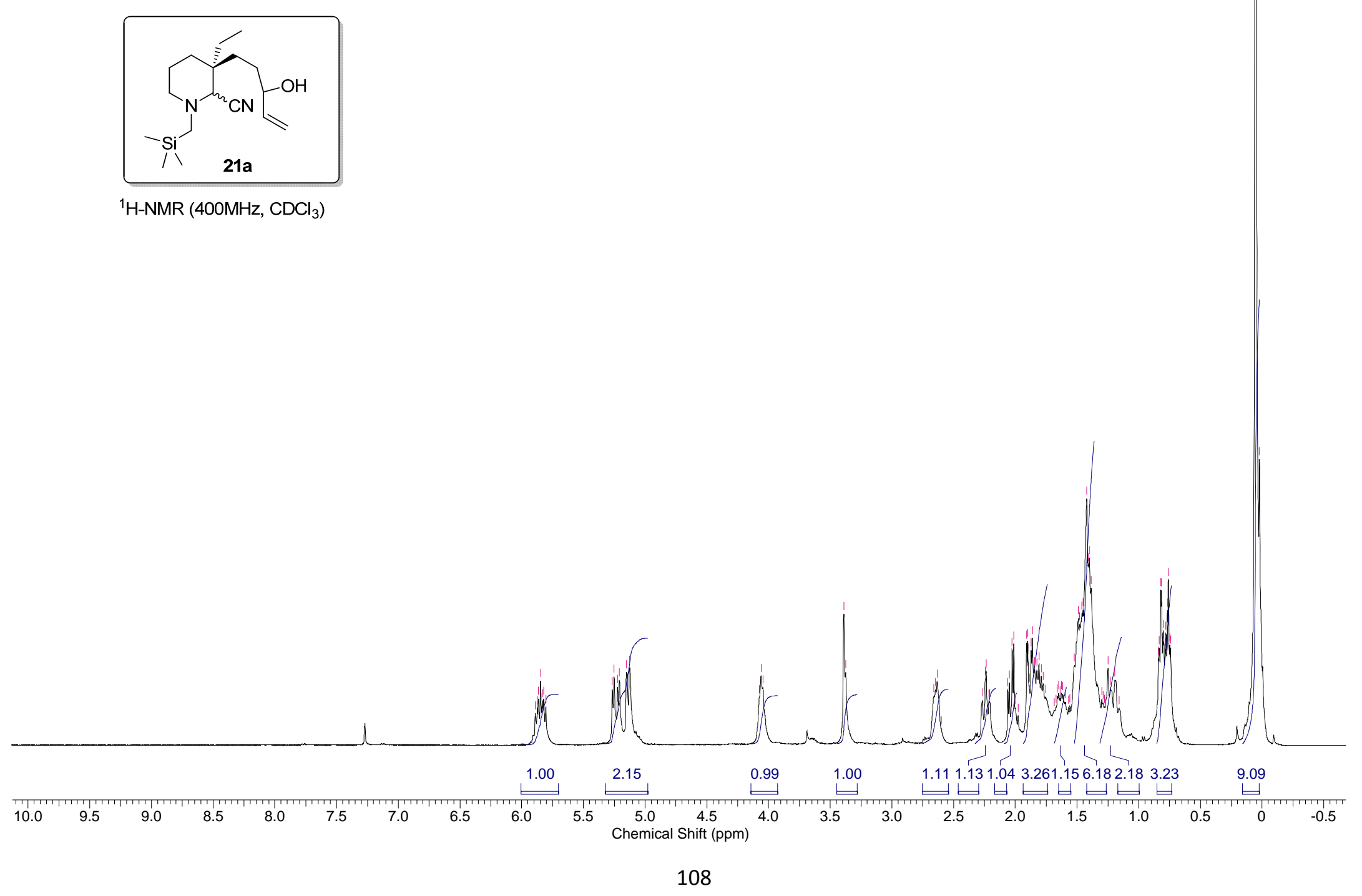

${ }^{1} \mathrm{H}-\mathrm{NMR}\left(400 \mathrm{MHz}, \mathrm{CDCl}_{3}\right)$

1 


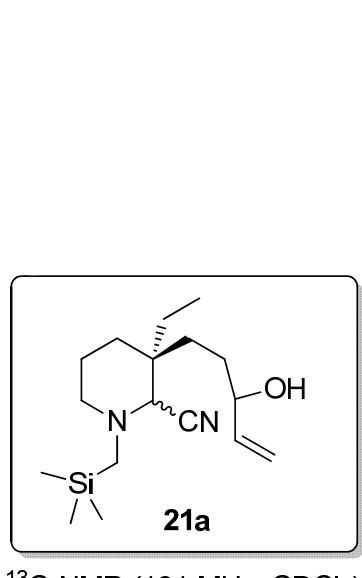

${ }^{13} \mathrm{C}-\mathrm{NMR}\left(101 \mathrm{MHz}, \mathrm{CDCl}_{3}\right)$

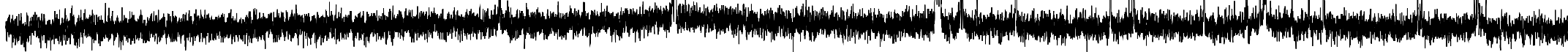

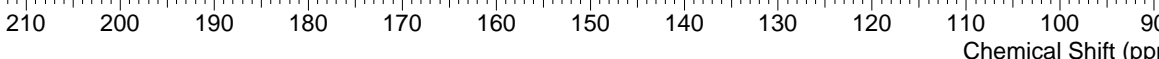

70

$20 \quad 10$ 


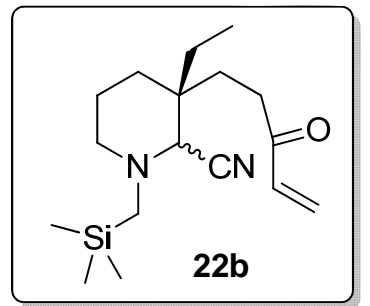

${ }^{1} \mathrm{H}-\mathrm{NMR}\left(400 \mathrm{MHz}, \mathrm{CDCl}_{3}\right)$

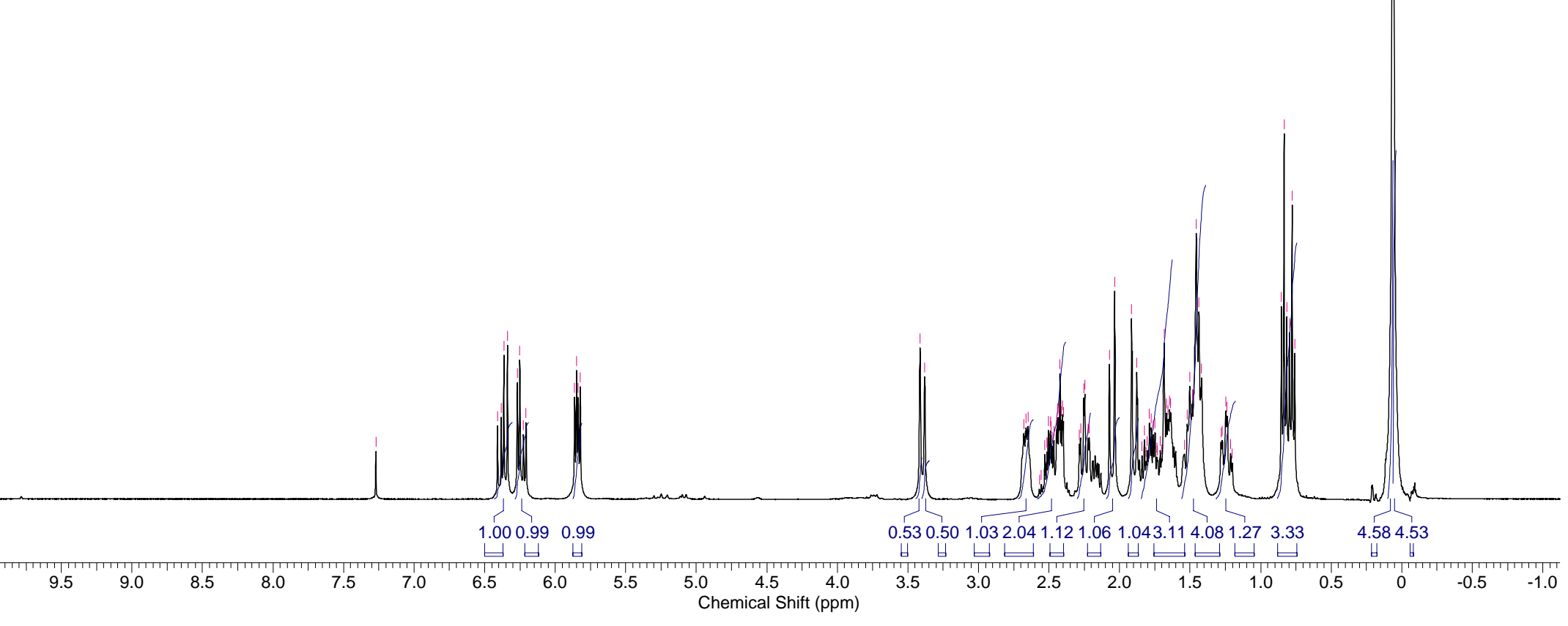


skb_enoneCN 2202.003.001.1r.esp

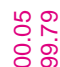

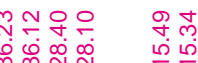

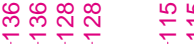

CHLOROFORM-d

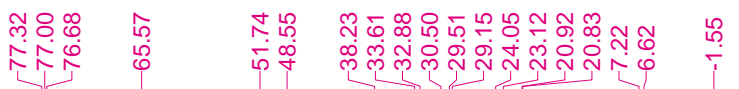

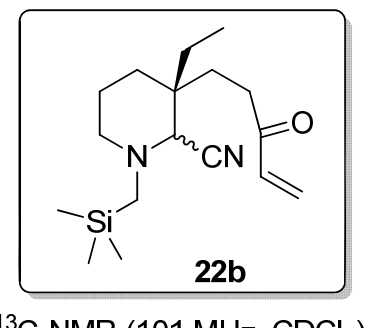

${ }^{13} \mathrm{C}-\mathrm{NMR}\left(101 \mathrm{MHz}, \mathrm{CDCl}_{3}\right)$

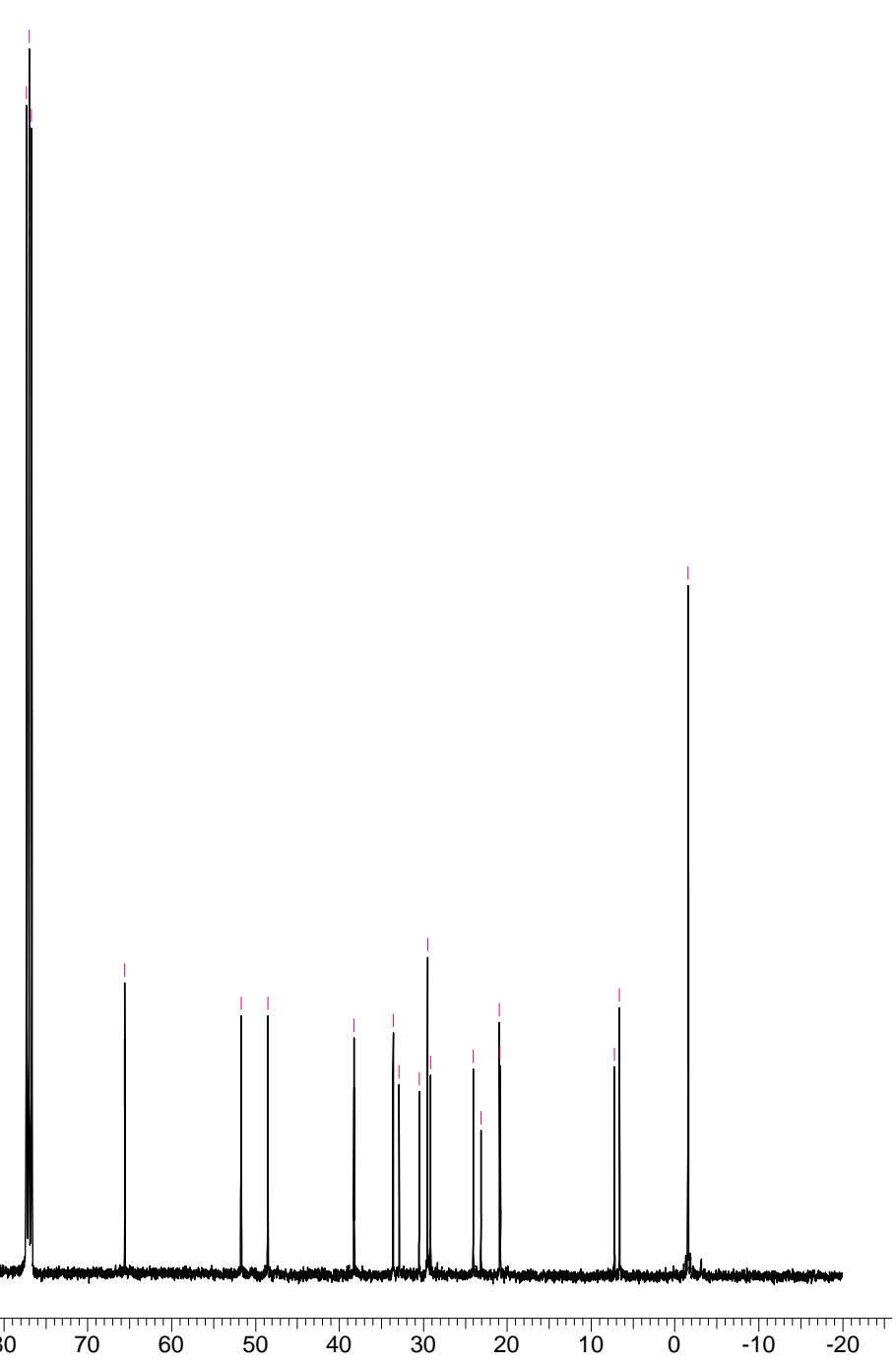
$\begin{array}{llllllllllll}220 & 210 & 200 & 190 & 180 & 170 & 160 & 150 & 140 & 130 & 120 & \begin{array}{l}110 \\ \text { Chemical Shift (ppm) }\end{array}\end{array}$ 


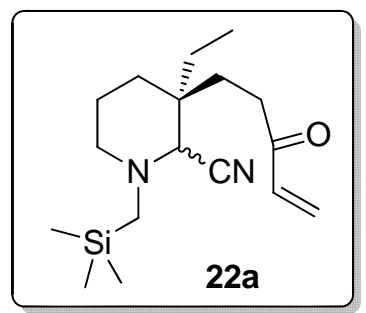

${ }^{1} \mathrm{H}-\mathrm{NMR}\left(400 \mathrm{MHz}, \mathrm{CDCl}_{3}\right.$ )

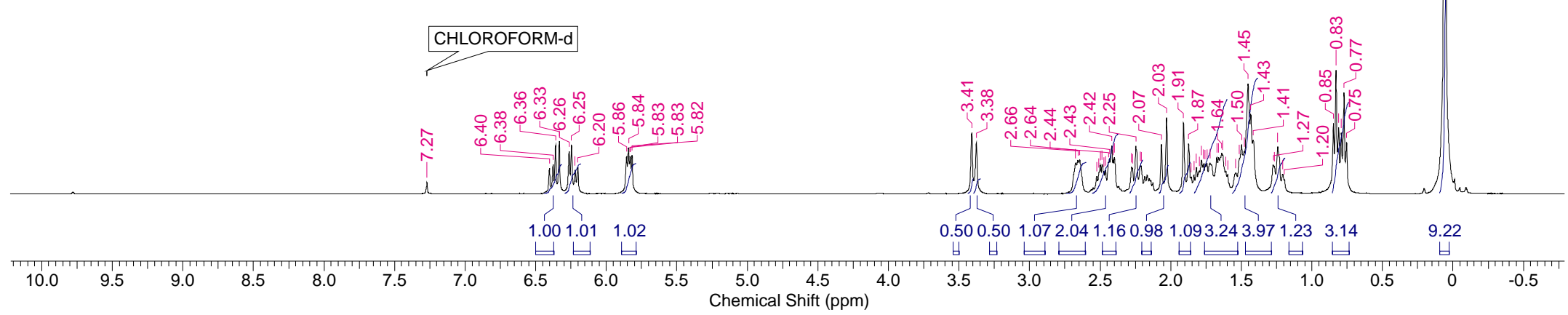




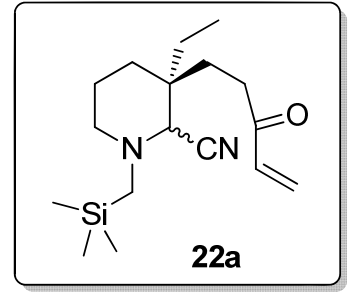

${ }^{13} \mathrm{C}-\mathrm{NMR}\left(101 \mathrm{MHz}, \mathrm{CDCl}_{3}\right)$

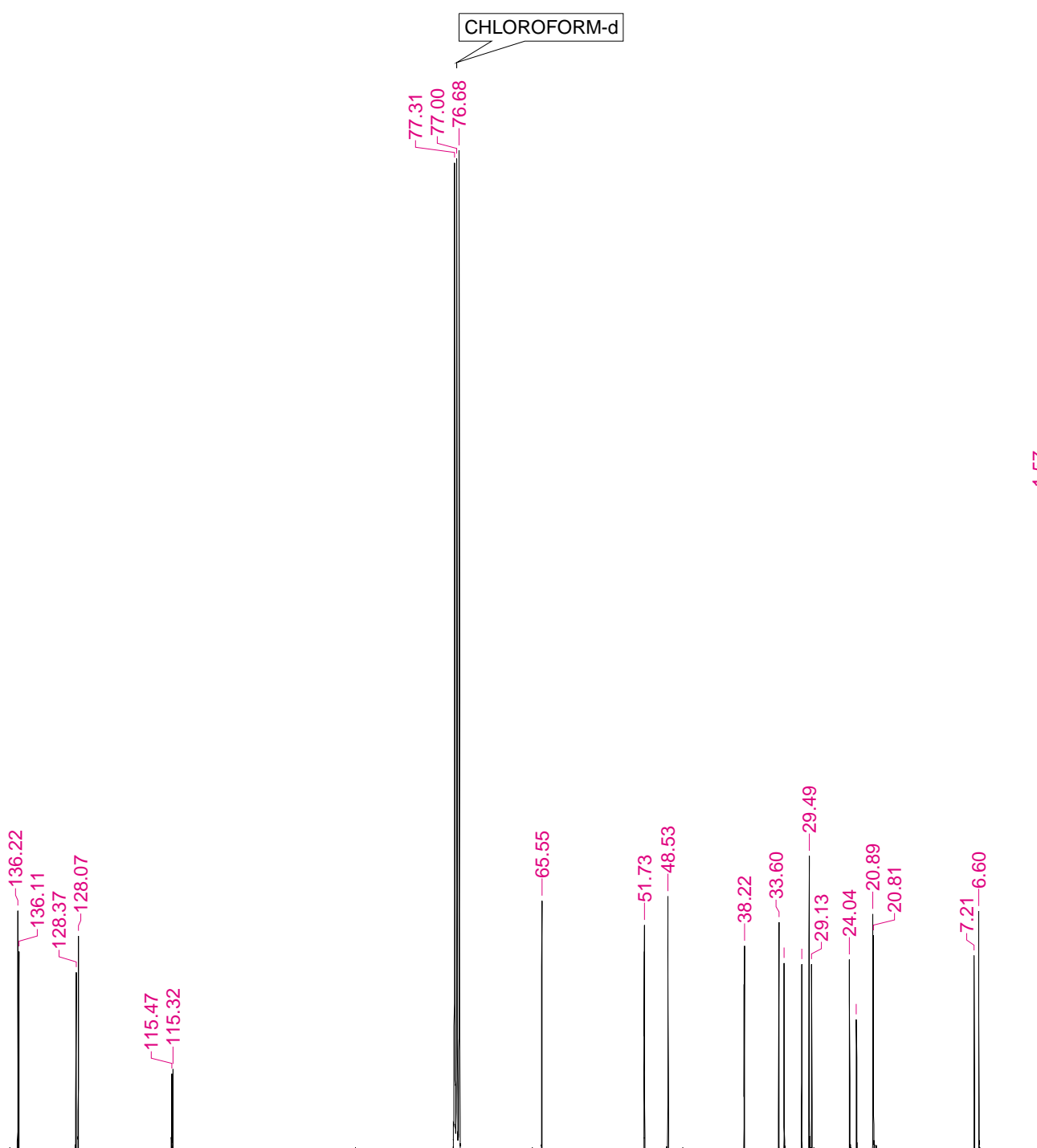

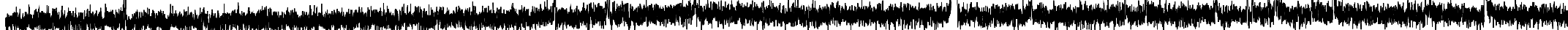

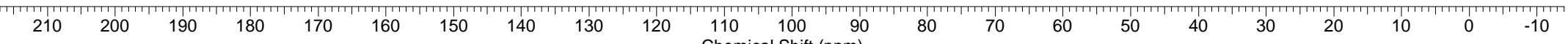




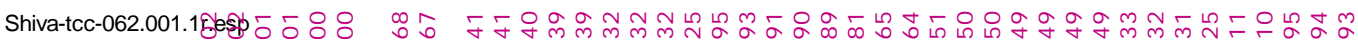
minmm 少
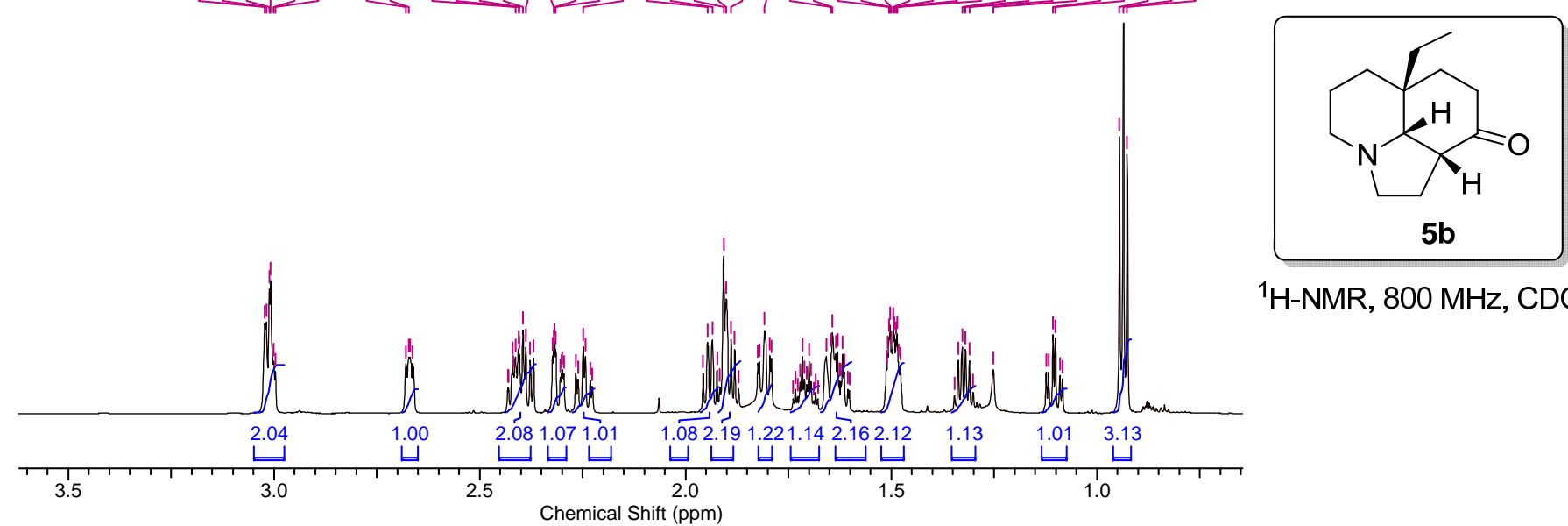

${ }^{1} \mathrm{H}-\mathrm{NMR}, 800 \mathrm{MHz}, \mathrm{CDCl}_{3}$

CHLOROFORM-
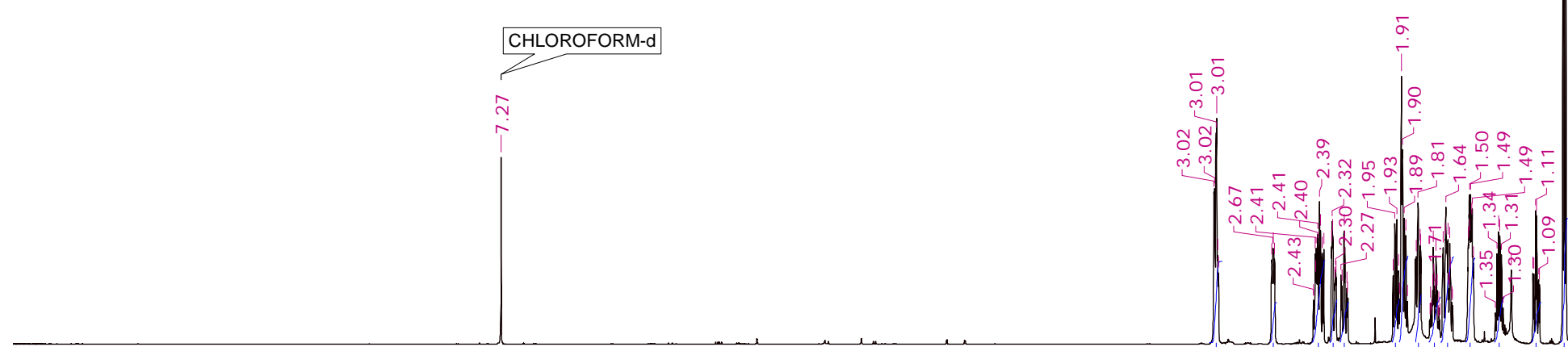

2.041 .002 .081 .071 .011 .082 .191 .221 .142 .162 .121 .131 .013 .13
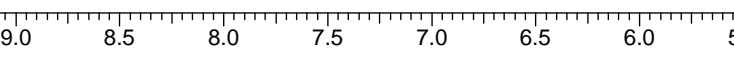

$\begin{array}{lll}5.5 & 5.0 & 4.5 \\ \text { Chemical Shift (ppm) }\end{array}$

Tागा1ा11 


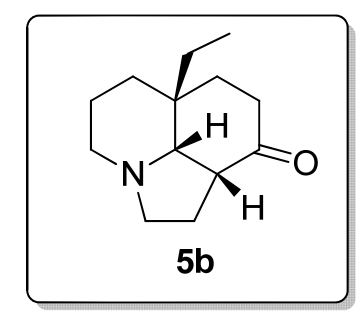

${ }^{13} \mathrm{C}-\mathrm{NMR}, 101 \mathrm{MHz}, \mathrm{CDCl}_{3}$ 


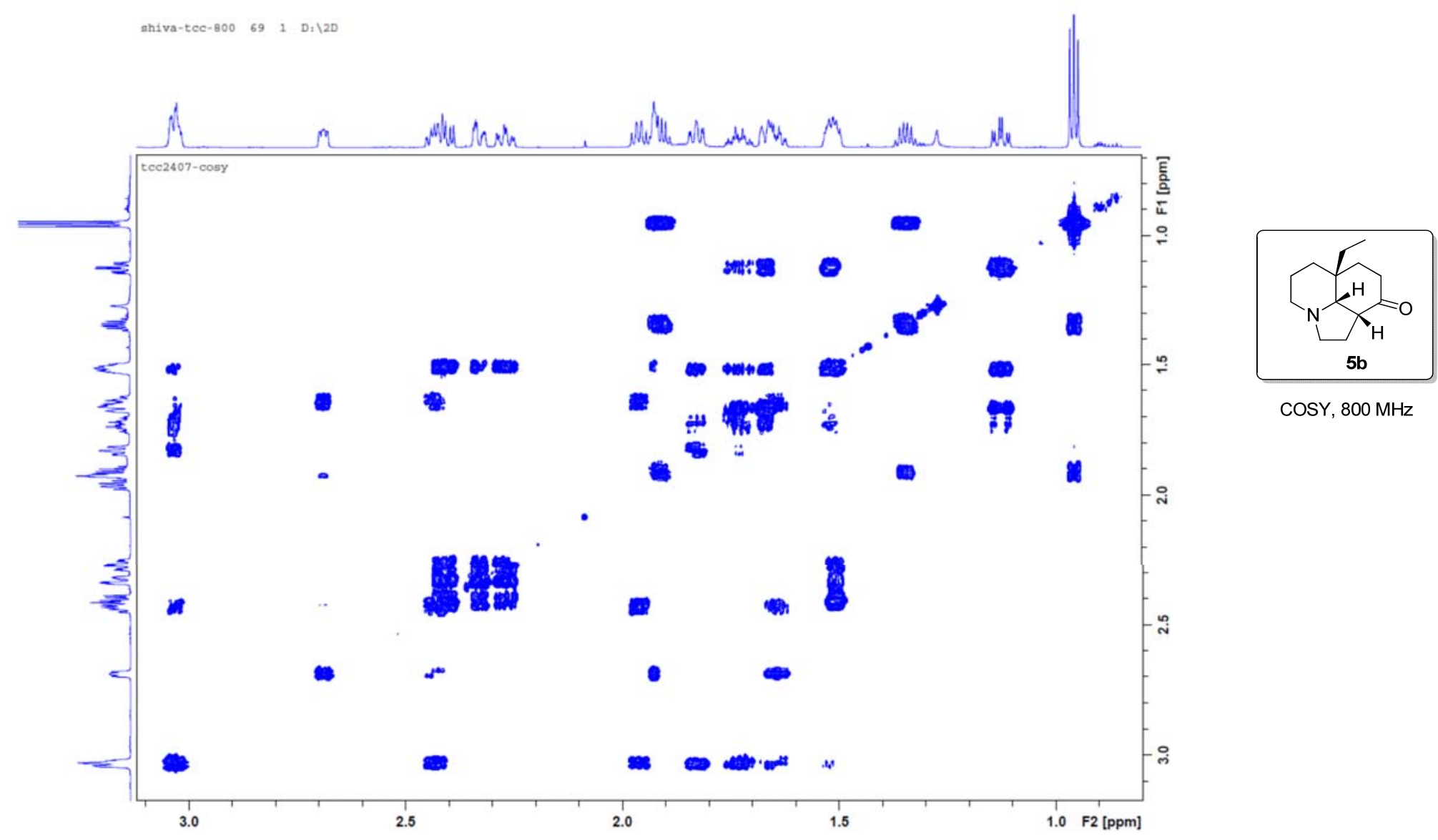


NOESY of $\mathbf{5 b}(800 \mathrm{MHz})$

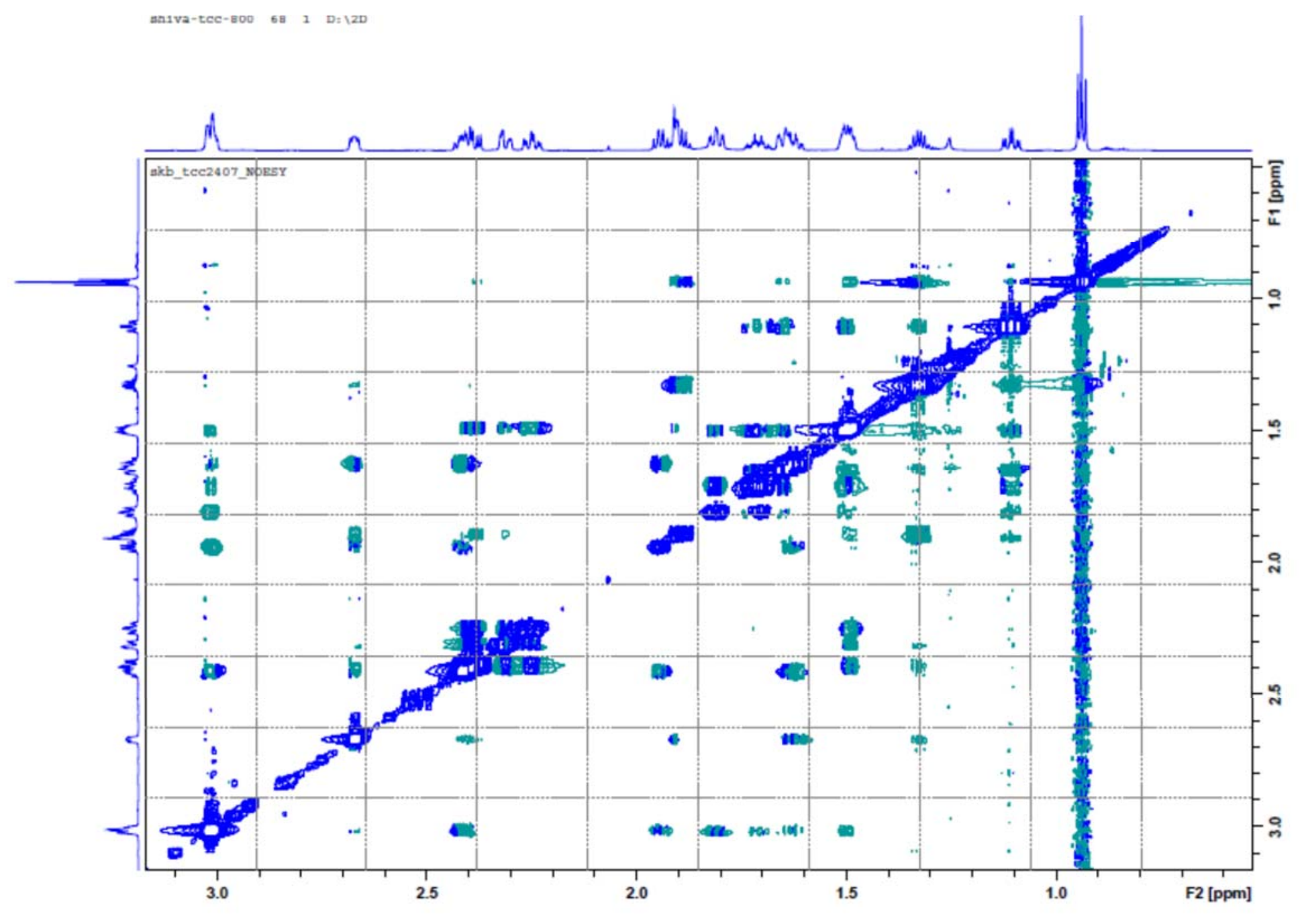




\section{NOESY correlation of $\mathbf{5 b}$}

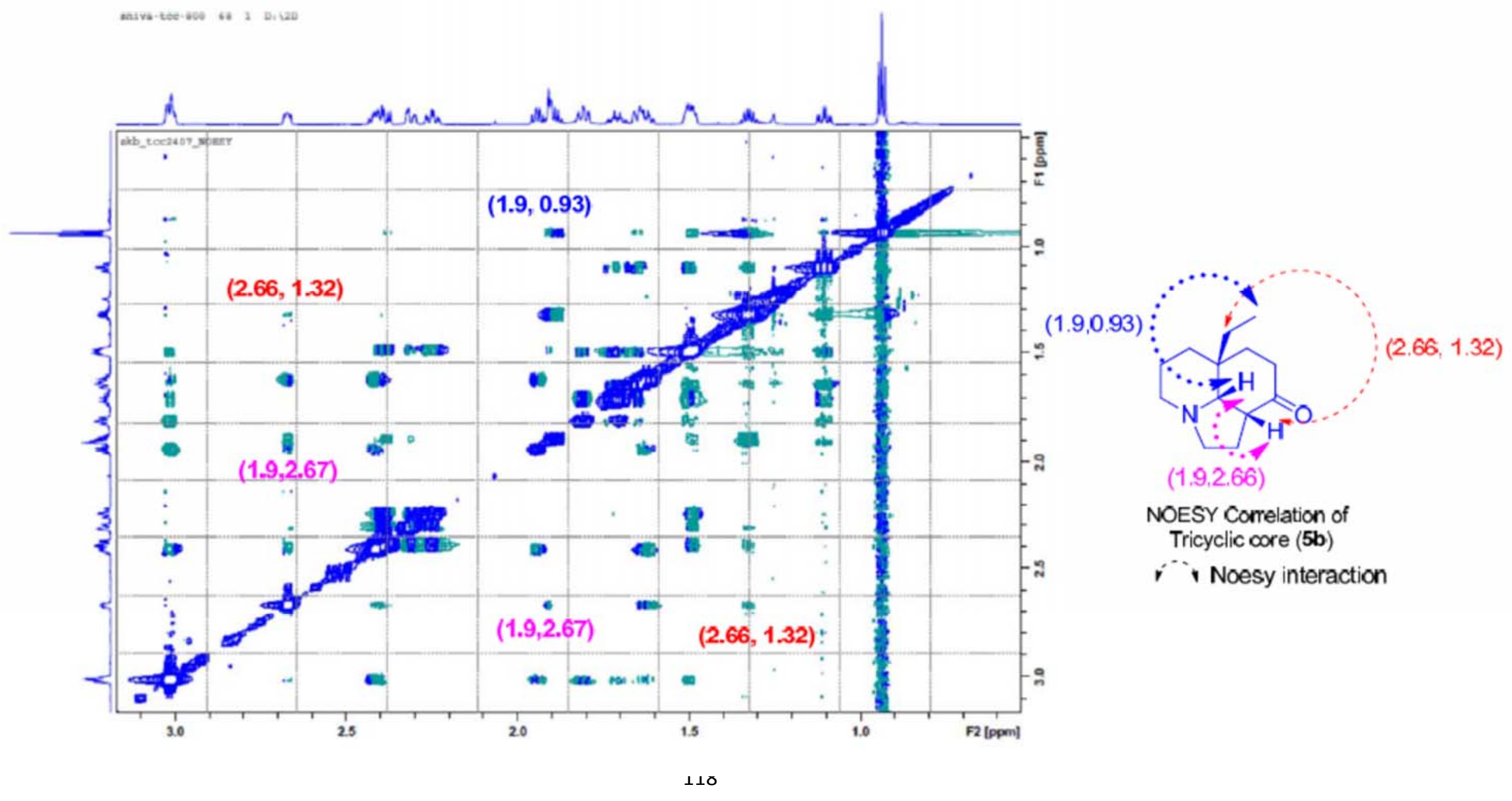




\section{HSQC of $5 b(800 \mathrm{MHz})$}

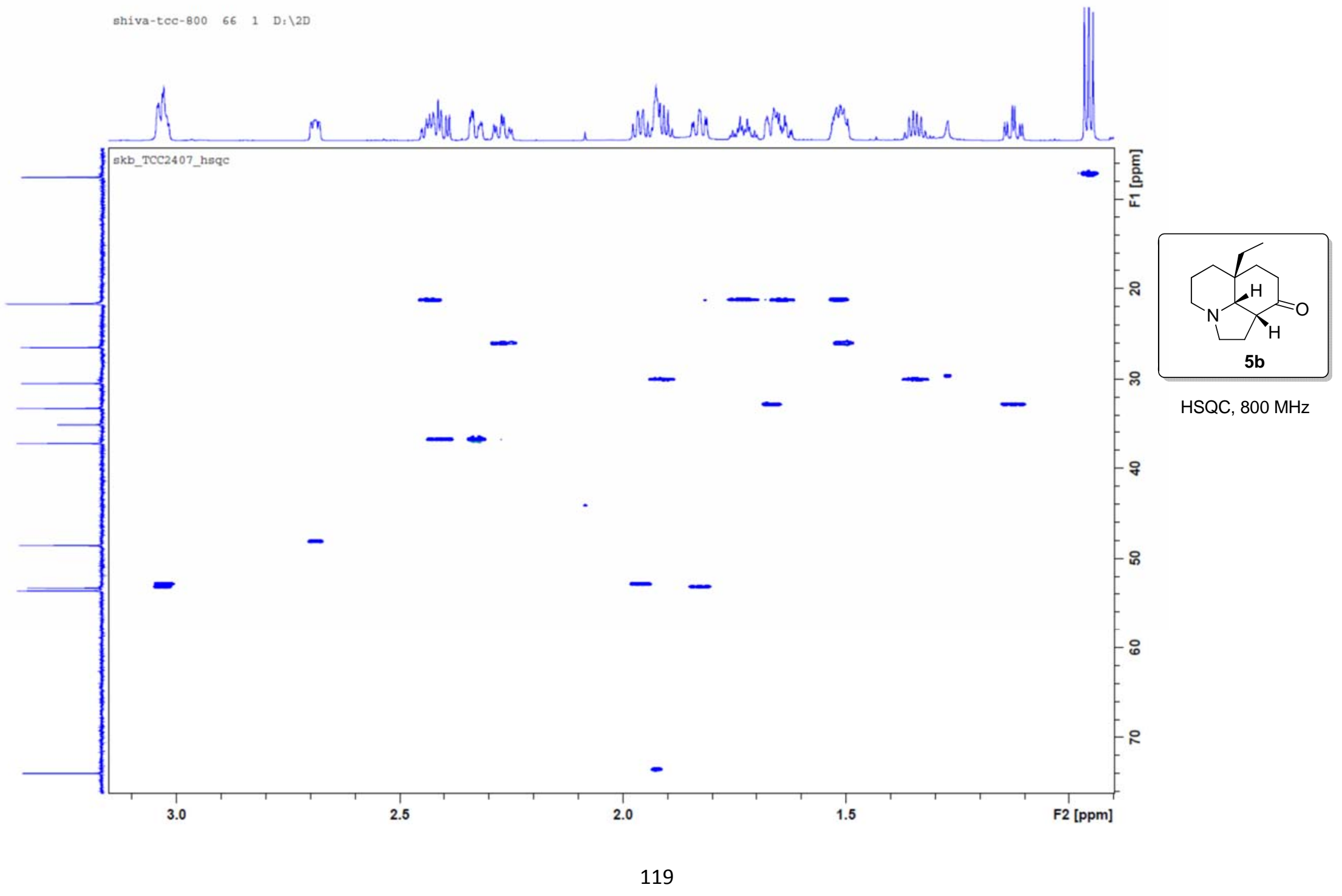


SHIVA-400.021.001.1r.esp
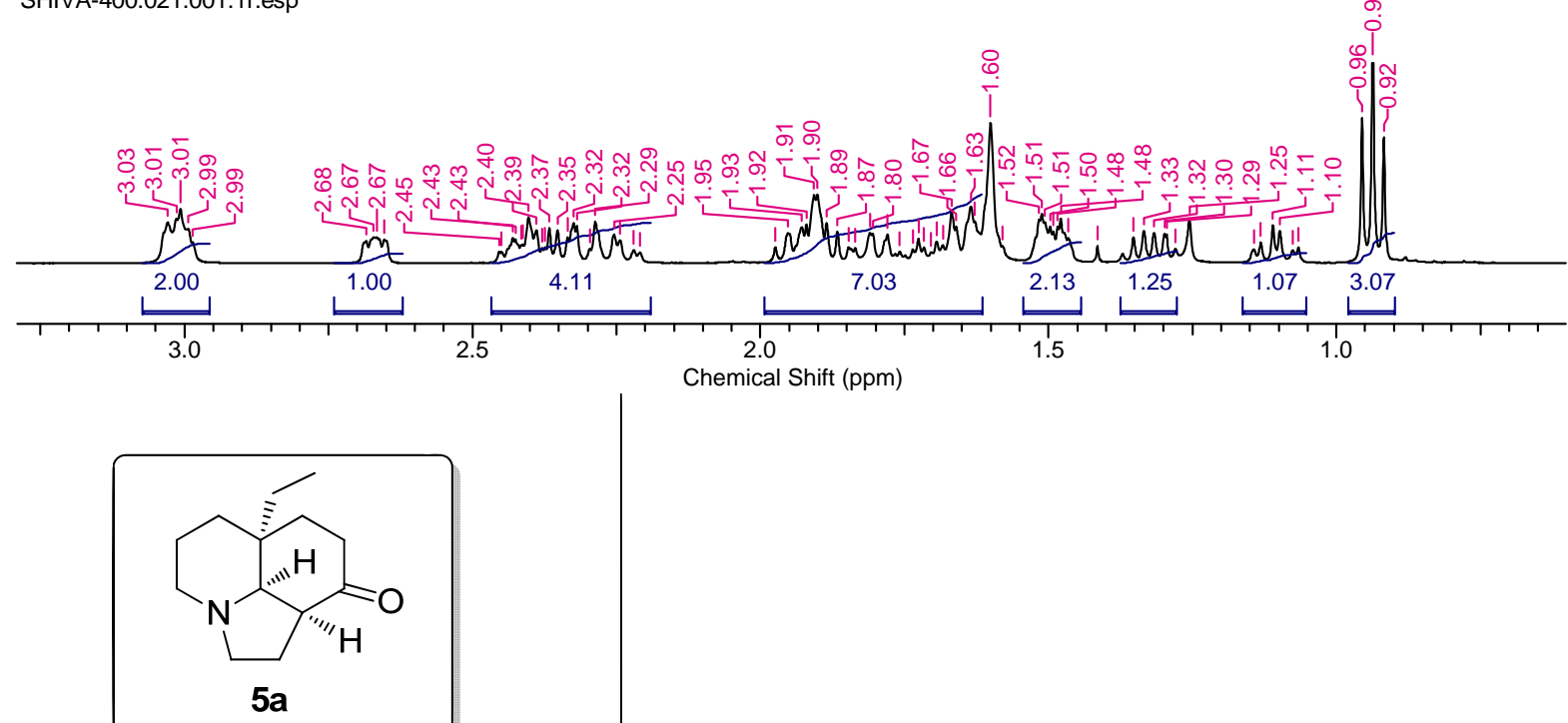

${ }^{1} \mathrm{H}-\mathrm{NMR}, 400 \mathrm{MHz}, \mathrm{CDCl}_{3}$

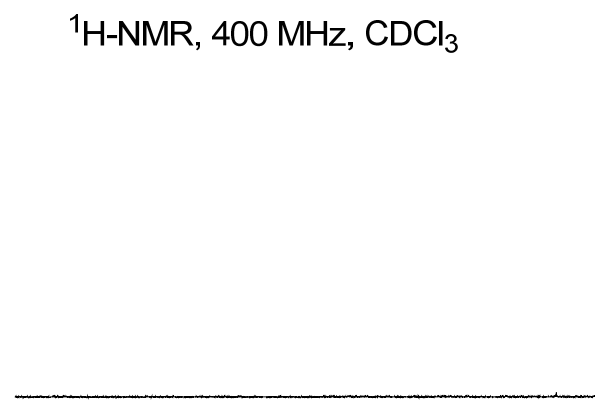

$\begin{array}{llll}2.00 & 1.00 & 4.11 & 7.03 \\ 2.13 & 1.25 & 1.073 .07\end{array}$

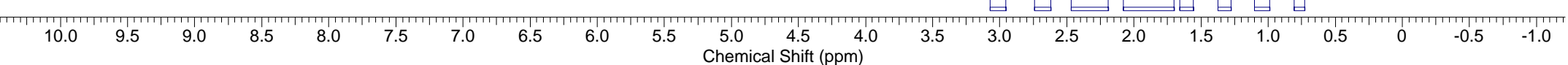




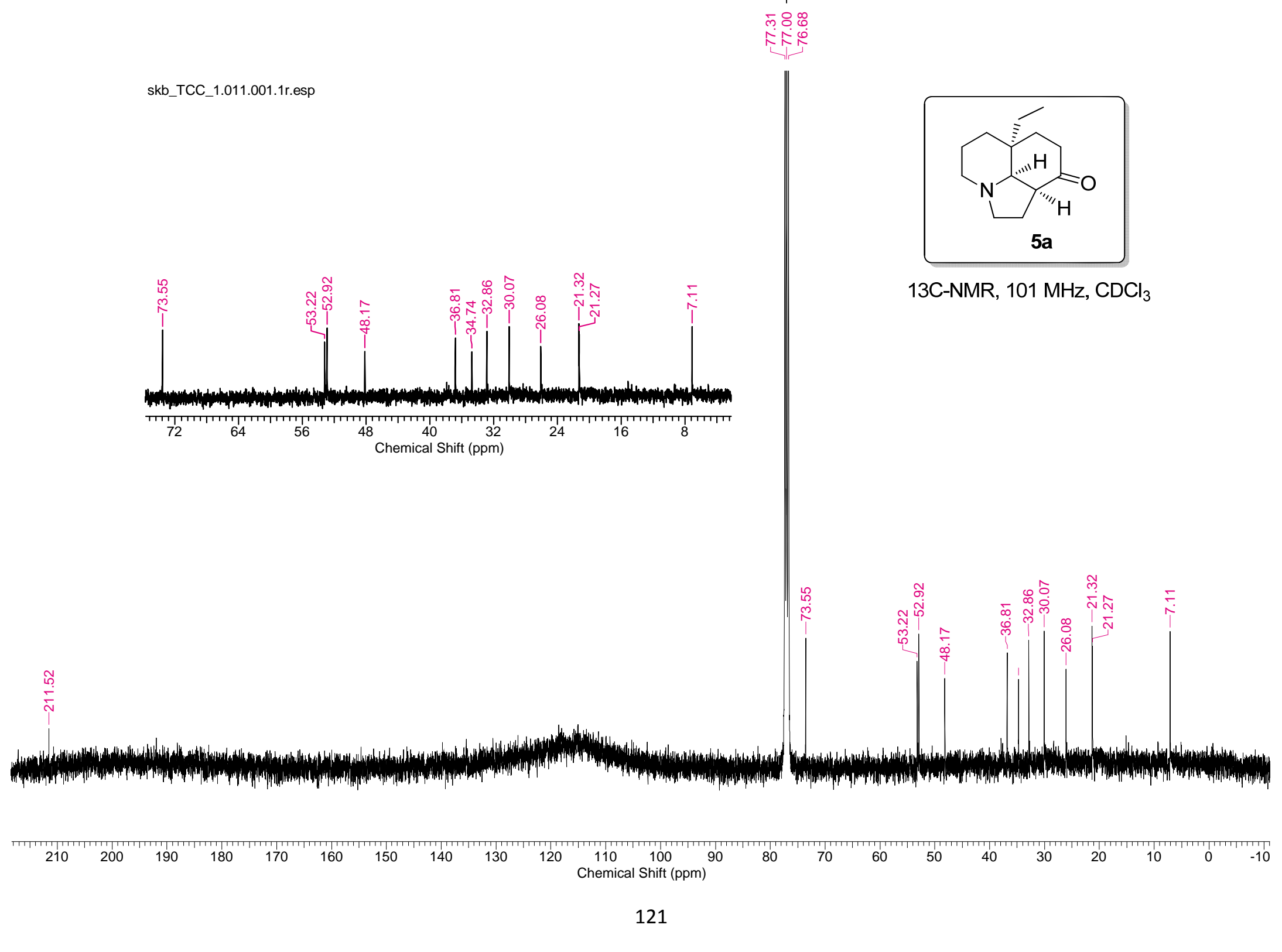




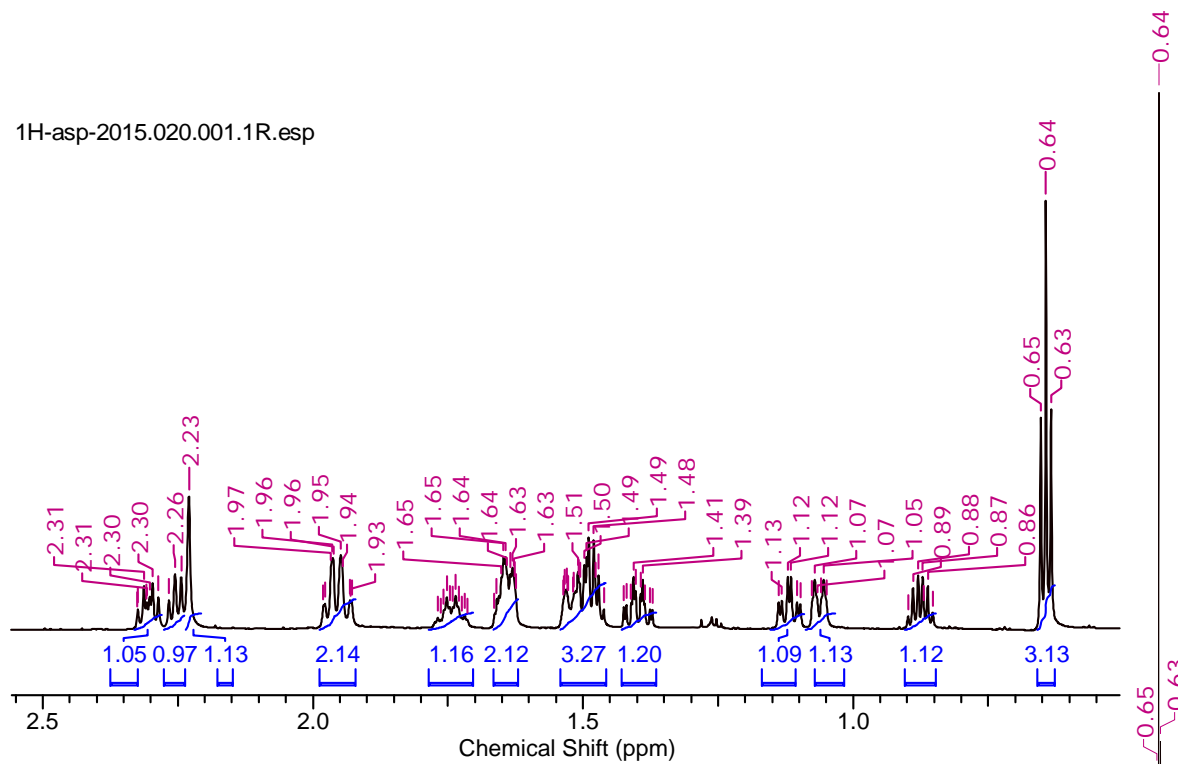

CHLOROFORM-d

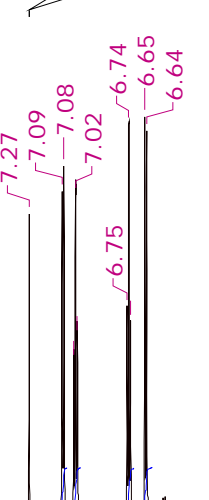

1.001 .010 .981 .01

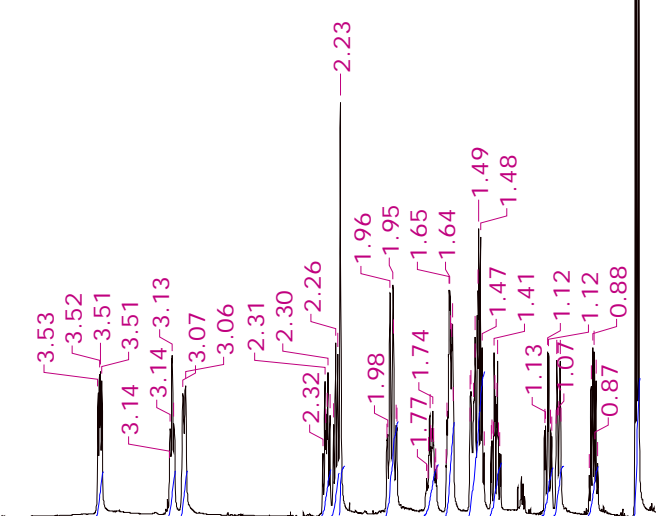

1.011 .031 .031 .050 .971 .132 .141 .162 .123 .271 .201 .091 .131 .123 .13 
13c-asp-2015.026.001.1r.esp

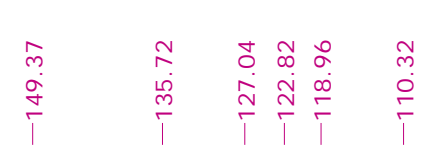

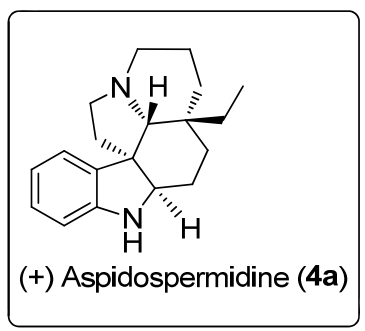

${ }^{13} \mathrm{C}-\mathrm{NMR}, 201 \mathrm{MHz}, \mathrm{CDCl}_{3}$
CHLOROFORM-d

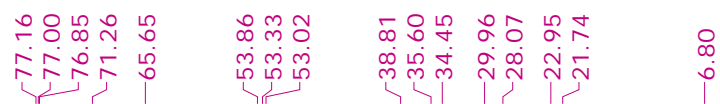

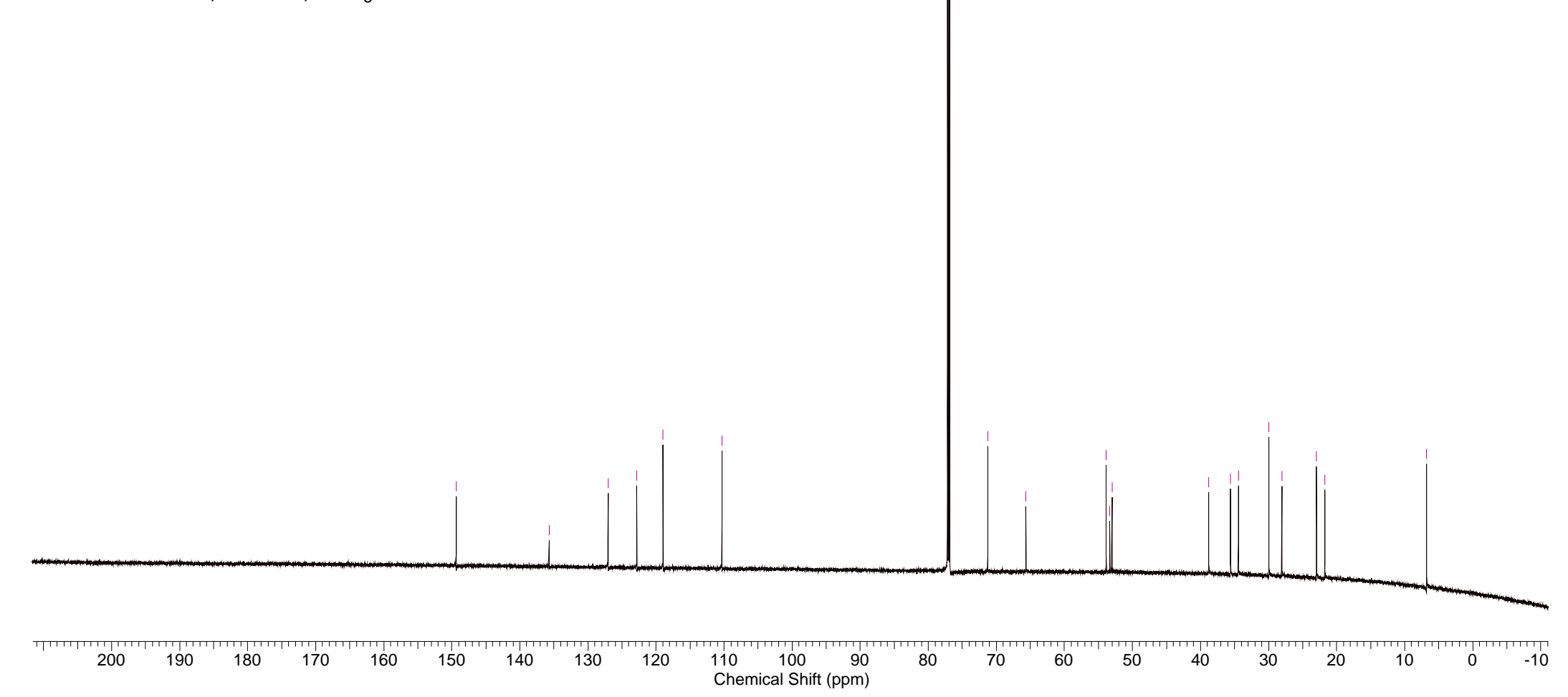


COSY of (+)- Aspidospermidine.

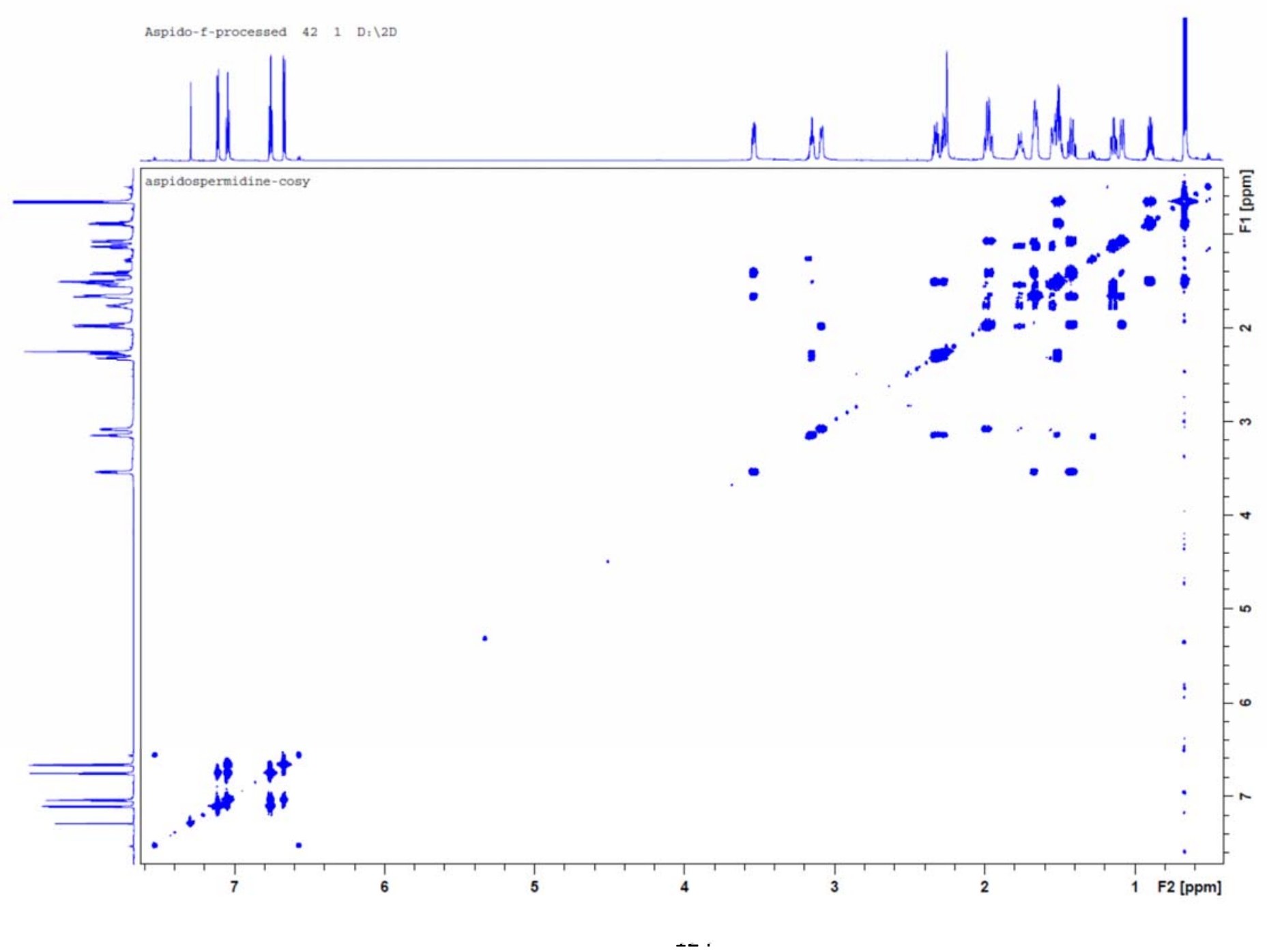


NOESY of (+)- Aspidospermidine

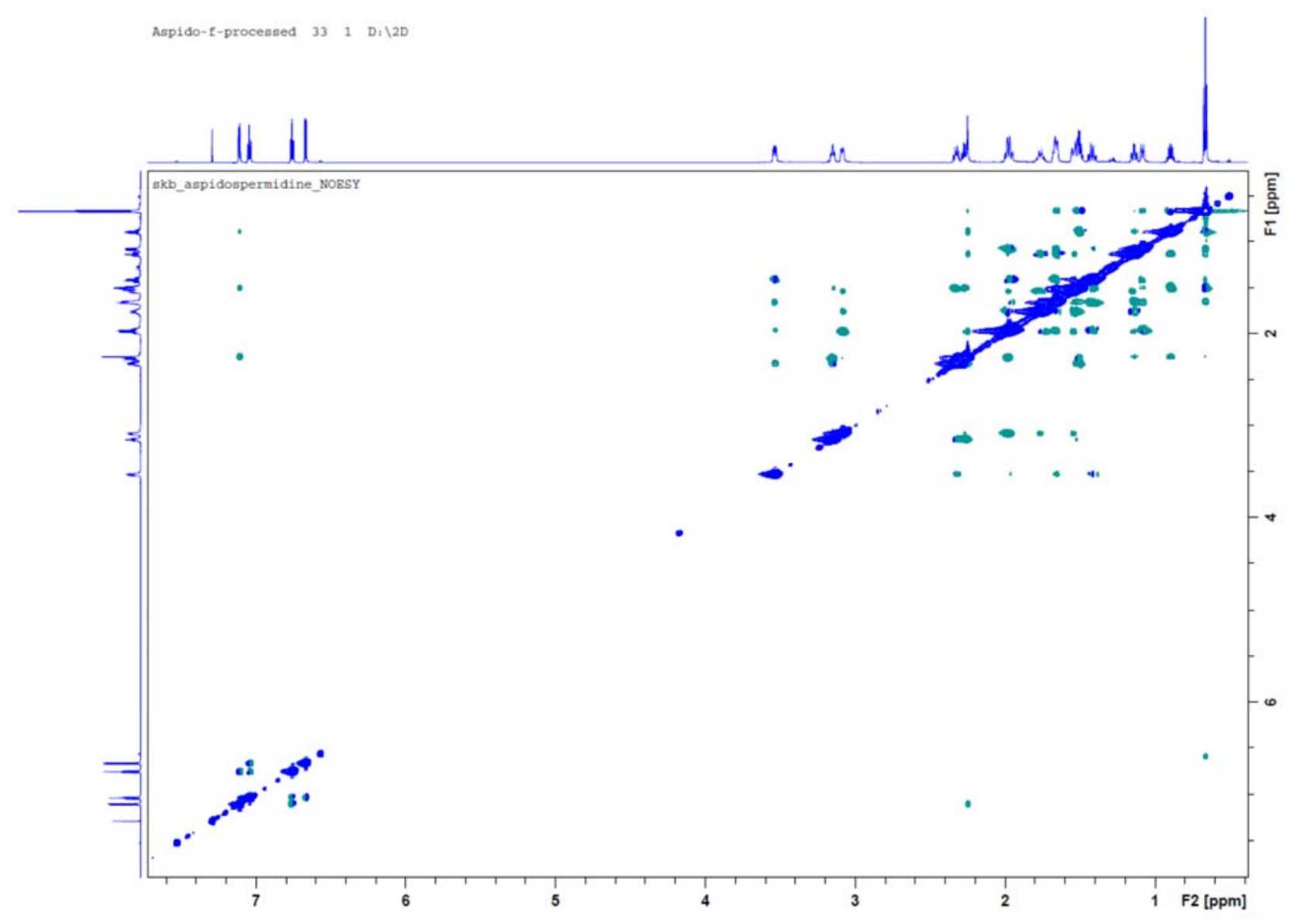


NOESY of (+)- Aspidospermidine (4a) (800 MHz), zoomed spectra:

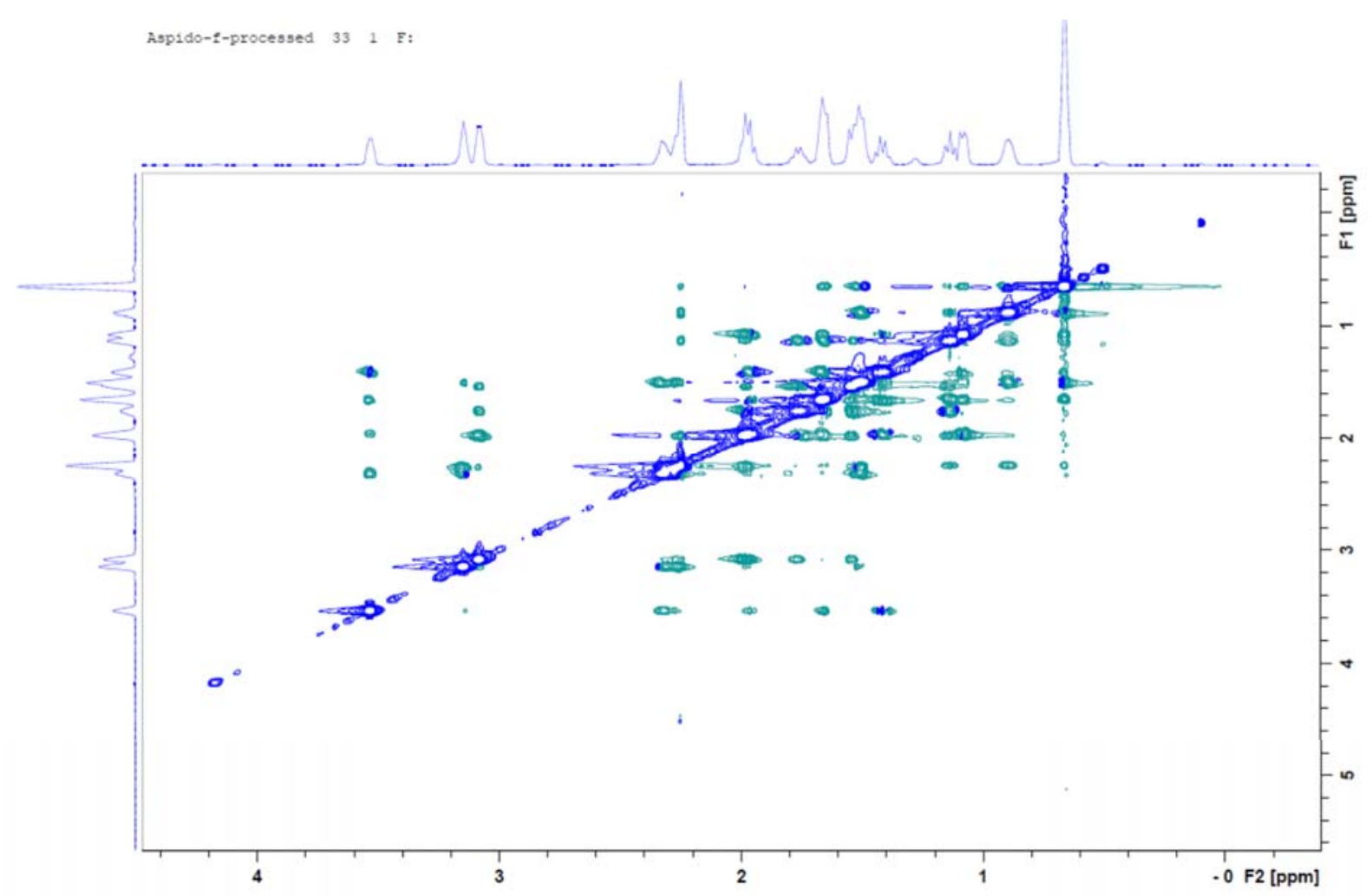


HSQC of (+)- Aspidospermidine(4a) (800 MHz):

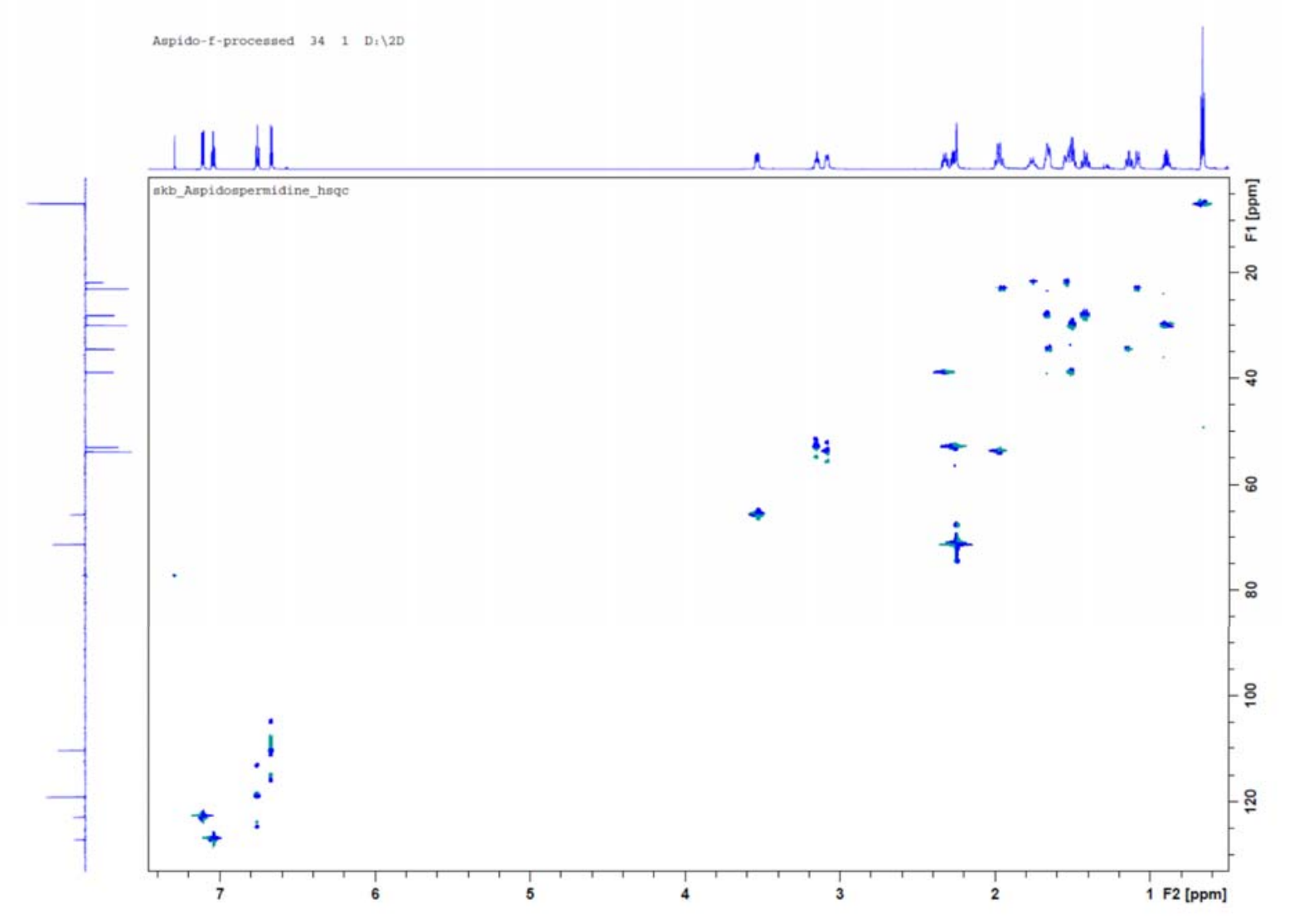


HMBC of (+)-Aspidospermidine (4a) (800 MHz):

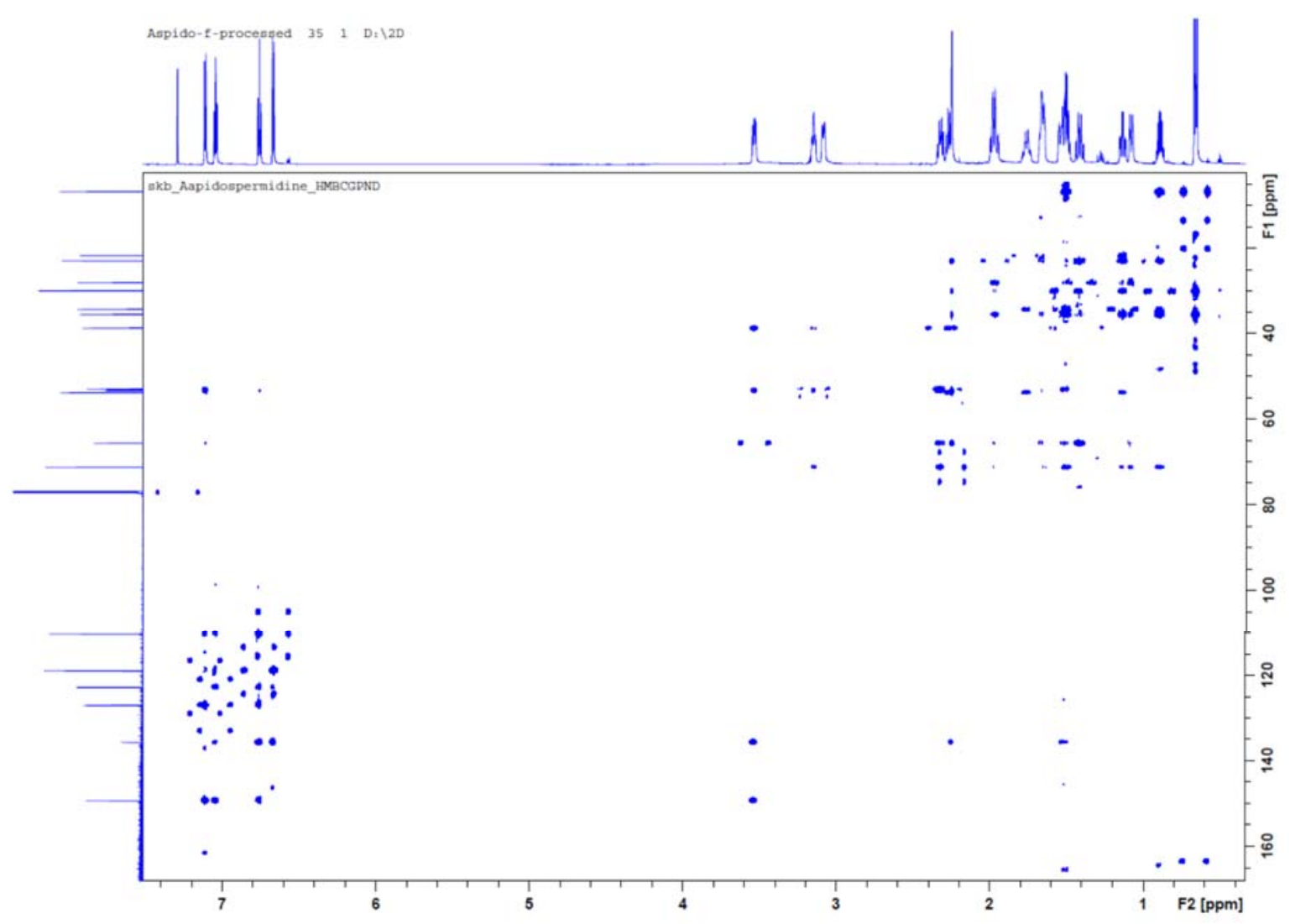

